\title{
DEVELOPMENT AND EVALUATION OF CUEING SYMBOLOGY FOR ROTORCRAFT OPERATIONS IN DEGRADED VISUAL ENVIRONMENT (DVE)
}

\author{
by \\ Vamshi Chittaluri \\ Bachelor of Engineering, Ryerson University (2016) \\ A thesis \\ presented to Ryerson University \\ in partial fulfillment of the \\ requirements for the degree of \\ Master of Applied Science \\ in the program of \\ Aerospace Engineering
}

Toronto, Ontario, Canada, 2019

C)Vamshi Chittaluri, 2019 


\begin{abstract}
AUTHOR'S DECLARATION
I hereby declare that I am the sole author of this thesis. This is a true copy of the thesis, including any required final revisions, as accepted by my examiners.
\end{abstract}

I authorize Ryerson University to lend this thesis to other institutions or individuals for the purpose of scholarly research.

I further authorize Ryerson University to reproduce this thesis by photocopying or by other means, in total or in part, at the request of other institutions or individuals for the purpose of scholarly research.

I understand that my thesis may be made electronically available to the public. 


\title{
DEVELOPMENT AND EVALUATION OF CUEING SYMBOLOGY FOR ROTORCRAFT OPERATIONS IN DEGRADED VISUAL ENVIRONMENT (DVE)
}

\author{
Vamshi Chittaluri \\ Master of Applied Science, Aerospace Engineering, Ryerson University, Toronto (2019)
}

\begin{abstract}
Degraded Visual Environment (DVE) is experienced when helicopters enter Inadvertent Instrument Meteorological Conditions (I-IMC). DVE can occur in the form of fog, night flight occurring naturally or when pilots try to land in unprepared (dusty, snowy) landing zones causing brownouts and whiteouts from rotor downwash. The Degraded Visual Environment Navigation Support (DVENS) project aimed to use a LiDAR to scan a specified Field of View (FOV) and range to identify a zone to be safe or unsafe for landing in a simulation capacity. A Head Down Display (HDD) with touch capabilities was used to provide Virtual Visual Meteorological Conditions (V-VMC), in which 3D conformal, 2D orthographic symbology is displayed. For the iterative design of the symbology, to diagnose and minimize pilot error in DVE the Taxonomic Framework for Aircrew Error evaluation is used. The framework allowed for a more direct design approach with clear objectives based on operational requirements and presenting an optimal workload for pilots. Maintaining the objective of showing the required information to the pilots while minimizing clutter is imperative as too much information can increase workload. Thus, the aircrew error Taxonomic framework helps identify the design goals required to neutralize pilot error leading to an efficient design. The Ryerson Mixed Immersive Motion Simulation (MIMS) lab's Fixed Base Simulator (FBS) and CAE, Presagis's HELI CRAFT were used as the simulation testing tools. Non-intrusive questionnaires such as NASA Task Load Index (TLX), Bedford and Cooper-Harper display rating scales were used to provide feedback and evaluate the display system. Multiple scales were used for validation of results and to measure the workload, stress, physical, psychological and time loads. This methodology and design were found to be extremely helpful in assisting pilots to land, take off in DVE and IIMC conditions.
\end{abstract}




\section{ACKNOWLEDGEMENTS}

First, I would like to thank my advisor, Dr. Joon Chung, for providing the opportunity to contribute to the Aerospace scientific community, and for his continued support and assistance through my graduate career. I want to thank members of the Ryerson Mixed Immersive Motion Simulation (MIMS) Lab for their support and help in research and project completion. I would also like to thank Dr. Craig Gregory, Sion Jennings, Derek 'Duff' Gowanlock of National Research Council Flight Research Laboratory (NRCFRL), Hermann Brassard, Nicolas Drapeau and Stephane Roy of CAE, Presagis Canada for their professional guidance, expertise and knowledge in the subjects of Software development and Rotorcraft operations in DVE. Lastly, I would like to thank my family and friends for their emotional and financial support through my educational pursuits. 


\section{TABLE OF CONTENTS}

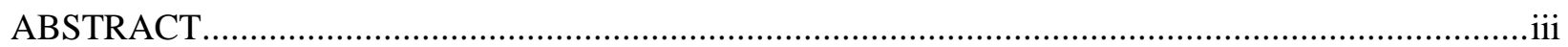

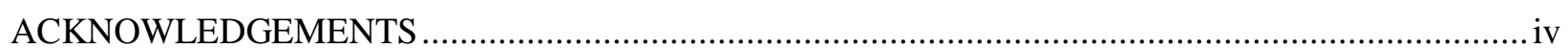

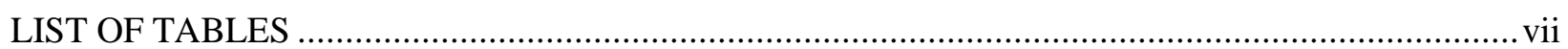

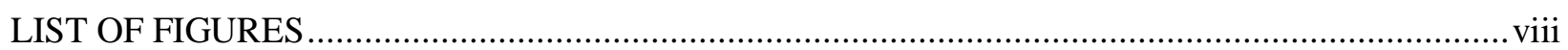

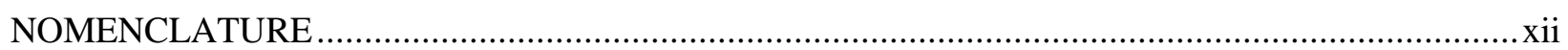

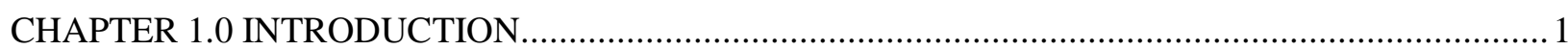

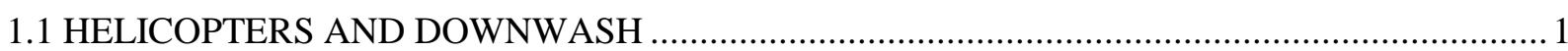

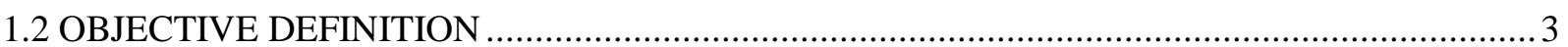

1.3 RESEARCH TOOLS: CAE, Presagis Software Package ............................................................. 4

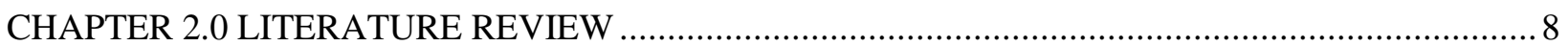

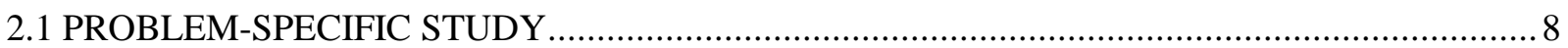

2.2 SITUATIONAL AWARENESS AND ILLUSIONS IN AVIATION ........................................

2.3 HELICOPTER OPERATIONS AND MANEUVERS ......................................................... 17

2.4 HELICOPTER FLIGHT DECKS, PAST, PRESENT AND THE FUTURE ..............................2

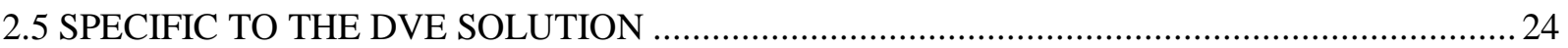

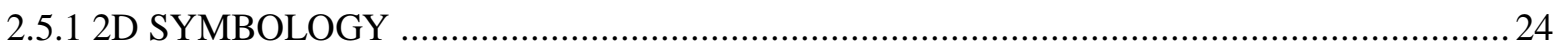

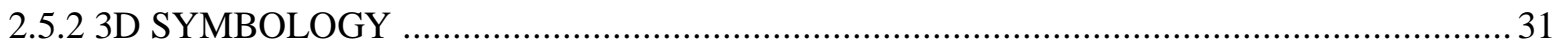

2.5.3 SECONDARY VIEW MODES AND TERRAIN TEXTURES …....................................... 33

CHAPTER 3.0 HUMAN FACTORS AND HUMAN MACHINE INTERFACING ............................. 41

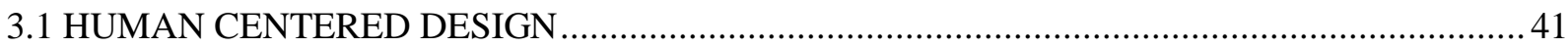

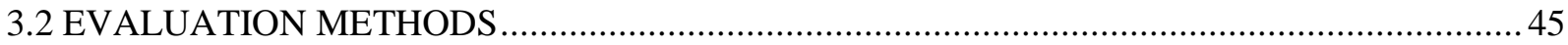

CHAPTER 4.0 SYMBOLOGY DEVELOPMENT AND DESIGN ................................................... 50

4.1 SYMBOLOGY REQUIREMENTS based on flight conditions ..............................................50

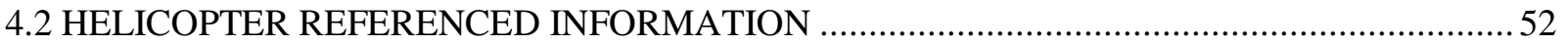

4.3 DESIGN OF SYMBOLOGY BASED ON ERROR TAXONOMY ........................................57

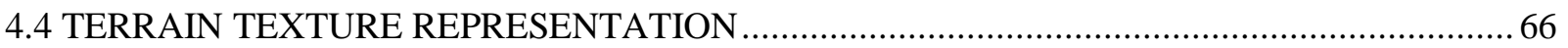

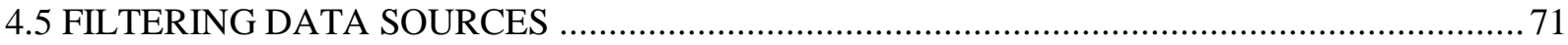

4.6 NAVIGATION VIA WAYPOINTS AND LANDING ASSIST …............................................ 73

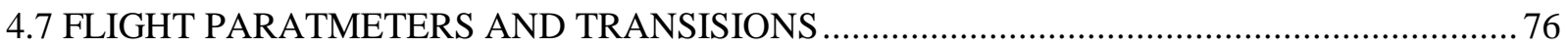

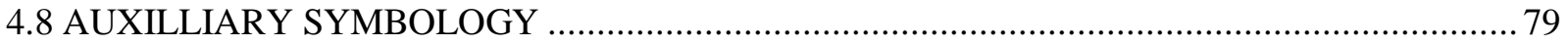

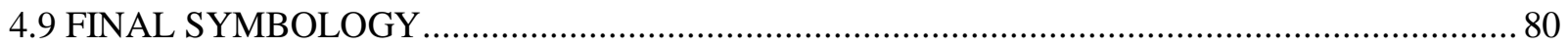

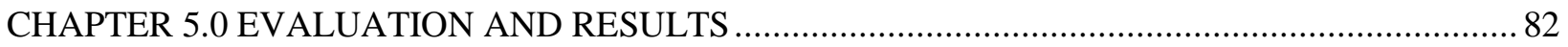

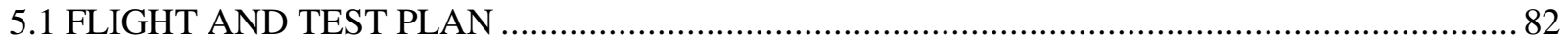


5.2 TABULATION AND EVALUATION OF RESULTS ..................................................... 90

5.3 NON-INTRUSIVE QUESTIONNARIE RESULTS ....................................................... 96

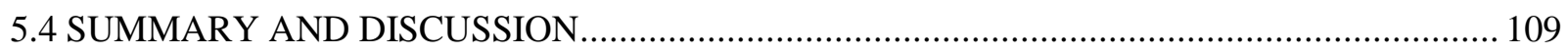

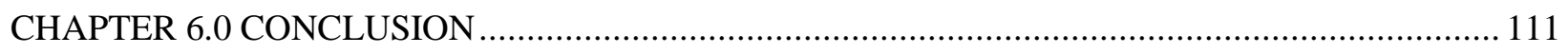

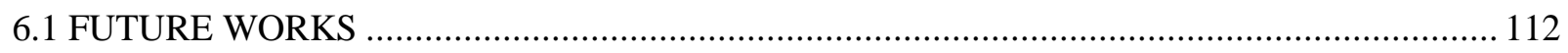

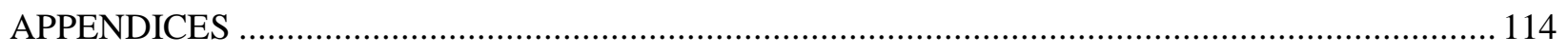

APPENDIX A: GRAPHS FOR HOVER PHASE ...................................................................... 114

APPENDIX B: CODES FOR DEVELOPMENT ….................................................................. 120

APPENDIX C: RYERSON ETHICS BOARD APPROVAL AND RELATING DOCUMENTS ...... 125

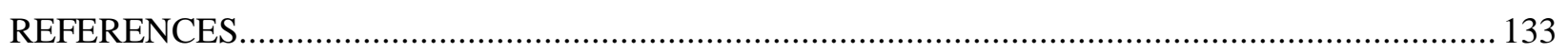




\section{LIST OF TABLES}

Table 1: Summary of common errors associated with the different landing methods. .......................... 19

Table 2: Table summarizing information correlating to indicator. ....................................................25

Table 3: Recommended color-use chart from MIL-STD-1787 ...................................................... 27

Table 4: Table of 2D orthographic display graphics and their function. ............................................52

Table 5: Summary of texture pattern tested with properties and issues experienced. ............................ 67

Table 6: Breakdown of participant experience information prior to flight testing. ................................ 88

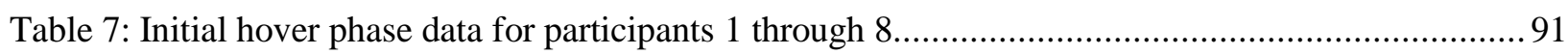

Table 8: Summary of results from the non-intrusive questionnaire results. ........................................... 109 


\section{LIST OF FIGURES}

Figure 1: A UH-1 Huey helicopter landing in Vietnam into a battlefield [2] . ................................... 1

Figure 2: Illustration of recirculation in hover and forward motion, creating a DVE situation in a dusty landing area. (a) in hover taxi (b) transition to forward flight (c) Low speed forward flight (d) Higher speed forward flight [4].

Figure 3: A figure illustrating a brownout (Ch-47 Chinook) and a whiteout (VH-3 Sikorsky) due to

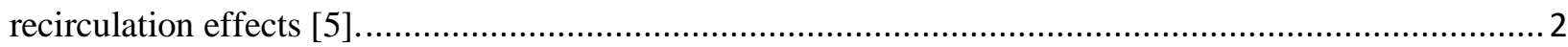

Figure 4: The CAE, Presagis HELI CRAFT helicopter simulator [13] ............................................. 5

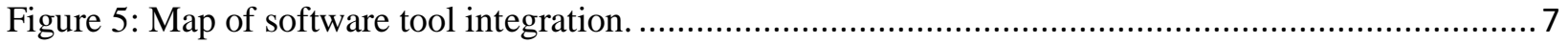

Figure 6: NATO suggested working areas for solution to solving DVE issues[8]. ............................... 8

Figure 7: Representation of Rotary wing non-Hostile losses (130 events) [9]. ....................................9

Figure 8: A helicopter completely immersed in a dust cloud [25] ................................................. 12

Figure 9: The Basic model of information processing adapted from Wicken and Flach (1988) [21]........14

Figure 10: Decision making model adapted from Wickens and Flach (1988)[21]. .............................14

Figure 11: Combined information processing and decision-making model. ...................................... 15

Figure 12:The taxonomic framework for assessing aircrew error adapted from O'Hare et al. (1994)[21]. 16

Figure 13: A rapid deceleration or quick stop landing depiction [26] ............................................. 17

Figure 14: Steep approach to hover method depiction [26] ..................................................... 17

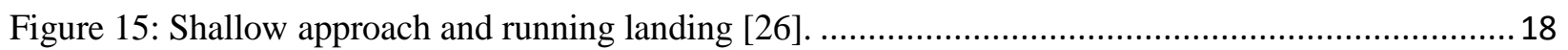

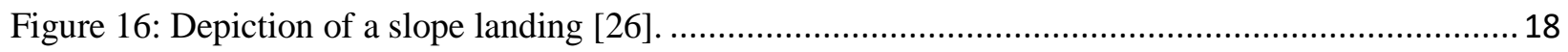

Figure 17: Picture of an old dial based UH-1B helicopter flight deck [27] ....................................... 21

Figure 18 Picture of a modern UH-1Y digital flight deck [27] ..................................................... 22

Figure 19:Depiction of a localizer setup that would be present at active airport runway [31] ................23

Figure 20: Depiction of glideslope setup that would be present at an active airport runway[31]............23

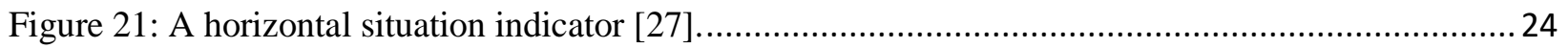

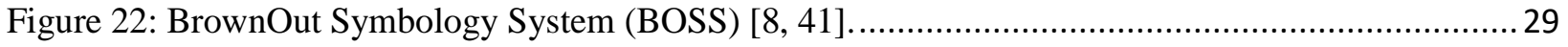

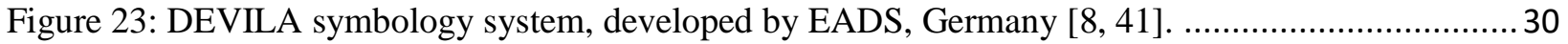

Figure 24: JEDEYE concept was developed by Elbit System together with DLR, Germany $[8,41] \ldots \ldots . . .30$

Figure 25: A 3D symbology display overlaid with helicopter information [42].................................. 31

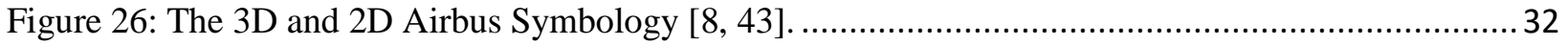

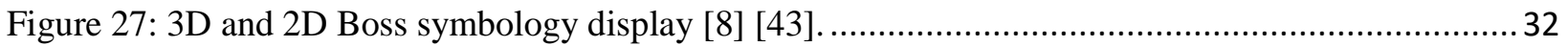

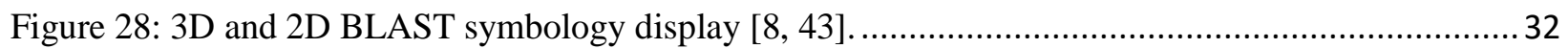


Figure 29: Visual representation of the three important factors, density, foreshortening and scaling, [45, 49]. 35

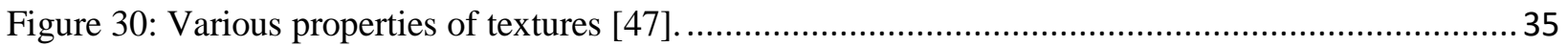

Figure 31: dual directional (D-1), uni-directional (D-2), and multi-directional(D-3) textures [50].......... 36

Figure 32:Overlaid uni-directional, dual directional and multi-directional textures pattern onto a 3D model to observe perception of detail for a 3D model with different textures from Figure 31[50]...........36

Figure 33: Texture patterns which were tested by Kim. S et al [51] ................................................. 37

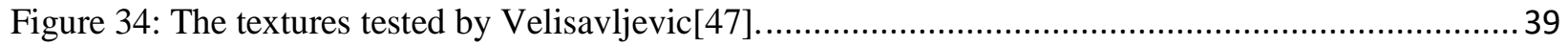

Figure 35: The Hierarchical Task Analysis Process Flowchart [54] .............................................. 42

Figure 36: Combined model of Taxonomic aircrew error and decision-making model.........................44

Figure 37: Hierarchical decision tree of the Bedford workload scale [62] ........................................ 46

Figure 38: The NASA-TLX index for pilot workload and stress by Hart and Staveland in 1988 [60]...... 48

Figure 39: The modified cooper harper display rating scale [63] ................................................ 49

Figure 40: Illustration of angle of attack, flight path angle and pitch angle derivation angles of Rotorcraft

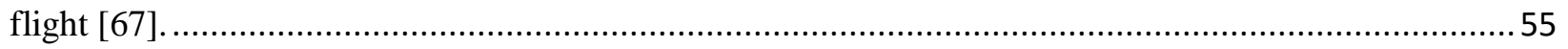

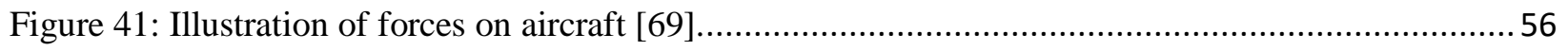

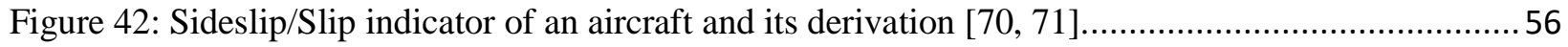

Figure 43: The taxonomic aircrew error framework used to provide solution to inability in discretizing

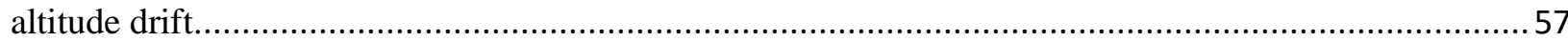

Figure 44: The taxonomic aircrew error framework used to provide solution to lack of lateral drift cue.. 58 Figure 45: The taxonomic aircrew error framework used to provide solution to inability in discretizing

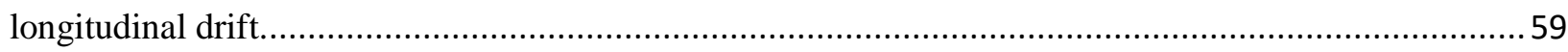

Figure 46: The taxonomic aircrew error framework used to provide solution to inability in discretize

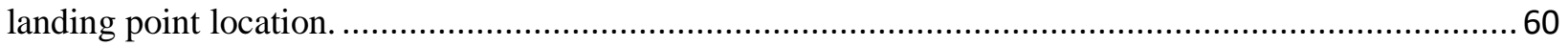

Figure 47: The taxonomic aircrew error framework used to provide solution to inability in discretize terrain information.

Figure 48: The taxonomic aircrew error framework used to provide solution to inability in discretize approach direction.

Figure 49: The Hover phase symbology developed using Presagis, CAE VAPS XT at Ryerson University.

Figure 50: The Cruise phase symbology developed using Presagis, CAE VAPS XT at Ryerson

University. 64

Figure 51: The 3-D symbology derived from the taxonomic aircrew error framework. 64 
Figure 52: The DVENS display with the forward view and top down view mode. in the forward view MODE, the rotor disk representation is show circled in red. The red squares in the Forward display correlate to the van shown in the otw. 64

Figure 53: The 3D and 2D symbology with obstacle information shown in one window, the right side shows the otw view. 65

Figure 54: Terrain texture that were tested during flight testing. .66 Figure 55: Three example textures (plaid pattern (A) two dimensional grids (B) and polka dots (C)) which underwent testing overlaid on a DEM while maintaining a low hover. 68

Figure 56: Two of the final textures selected from extensive flight tests, the SandMesh-Box and

SandMesh-Hex. .68

Figure 57: The D-2 Sandmesh Hex texture overlaid on top of the terrain at approx. $10 \mathrm{Ft}$ and 10 knots. . 69 Figure 58: The D-1 Sandmesh Box texture overlaid on top of the terrain at approx.10 Ft and 10 knots...69 Figure 59: Example of a banded rainbow (A) type contour line elevation display and the chosen display, which follows a contour banded principle in a greyscale palette $(\mathrm{B})$................................................ 70

Figure 60: Spherical triangle solved by the law of haversines [75] .................................................. 74

Figure 61: Representation of range and deviation calculation. ...................................................... 75

Figure 62: Algorithm flowchart for hover/cruise symbology transition [78] ................................... 76

Figure 63: Algorithm flowchart for Altitude source transition between barometric and radar altitude [78].

Figure 64: Algorithm logic for rotation of the landing zone symbology [78]. ................................... 78

Figure 65: Auxiliary Symbology developed using Presagis, CAE VAPS XT at Ryerson University. ...... 79

Figure 66: The complete DVENS symbology with obstacle representation, for description of the labelled components please refer to table 4 in chapter 4.2 . .... 81

Figure 67: The PFD symbology used for the flight test.

Figure 68: The Symbology testing was performed on the Ryerson Fixed Base Simulator (FBS) [53] while the texture testing was performed on Presagis HELICRAFT. ..................................................... 83

Figure 69: Map of the flight plan, showing route to be followed by the participants. .84

Figure 70: Plot of time taken to achieve hover longitudinal speed by participants 1 through 8 with the PFD and 2D displays.

Figure 71: PLOT OF TIME TAKEN TO ACHIEVE HOVER vertical SPEED BY PARTICIPANTS 1 THROUGH 8 WITH THE PFD AND 2D DISPLAYS.

Figure 72:PLOT OF TIME TAKEN TO ACHIEVE HOVER VERTICAL SPEED BY PARTICIPANTS 1 THROUGH 8 WITH THE PFD AND 2D DISPLAYS...... .94 
Figure 73: Frustration experienced survey results by participants using PFD, 2D and 3D displays from NASA TLX frustration scale.

Figure 74: Self Performance survey results by participants using PFD, 2D and 3D displays from NASA

TLX self-performance scale.

Figure 75: Effort required survey results by participants using PFD, 2D and 3D displays from NASA

TLX effort scale.

Figure 76: Temporal demand experienced survey results by participants using PFD, 2D and 3D displays from NASA TLX temporal demand scale.

Figure 77: Physical demand experienced survey results by participants using PFD, 2D and 3D displays

from NASA TLX physical demand scale. 100

Figure 78: Mental demand experienced survey results by participants using PFD, 2D and 3D displays from NASA TLX mental demand scale. 101

Figure 79: The Cooper-Harper survey results by participants using PFD, 2D and 3D displays. 102

Figure 80: The Bedford workload scale survey results by participants using PFD, 2D and 3D displays.

Figure 81: The recorded landing points using the PFD display, with lateral and longitudinal deviation grids.

Figure 82: The recorded landing points using the 2D orthographic display, with lateral and longitudinal deviation grids.

Figure 83: The recorded landing points using the 3D Conformal display, with lateral and longitudinal deviation grids...... 106

Figure 84: Feedback from participants 1 and 2 for the SandMesh-Box texture 107

Figure 85: Feedback from participants 1 and 2 for the SandMesh-Hex texture 108

Figure 86: Feedback from participants 1 and 2 for the Checkerboard pattern texture. 108 Figure 87: The recorded landing points using the PFD, 2D orthographic and 3D Conformal displays, with lateral and longitudinal deviation grids. 110 


\section{NOMENCLATURE}

CAS - Cockpit Assistant System

CDB - Common Databases

CDI - Course Deviation Indicator

COTS - Commercial off the Shelf

DEM - Digital Elevation Map

DOF - Degree of Freedom

DVE- Degraded Visual Environment

FMS - Flight Management System

FOV - Field of View

HDD - Head Down Display

HFC - Human Factor Condition

HMD - Helmet Mounted Display

HMI - Human Machine Interface

HTA - Hierarchical Task Analysis

HUD - Heads Up Display

IFR - Instrument Flight Rules

ILS - Instrument Landing System

IMC/IIMC - Instrument Meteorological Conditions/Inadvertent Instrument Meteorological Condition

LIDAR - Light Detection and Ranging

MFD - Multifunctional Display

ND - Navigation Display

OTW - Out the Window

PFD - Primary Flight Display

SA - Situational Awareness

SLZ/SLP - Safe landing zone/Safe landing Point

SVS - Synthetic Vision Systems

VFR - Visual Flight Rules

VMC -Visual Meteorological Conditions

VTOL - Vertical Takeoff or Landing 


\section{CHAPTER 1.0 INTRODUCTION}

\subsection{HELICOPTERS AND DOWNWASH}

The first active operational helicopter was the German Focke-Wulf FW 61 which flew in 1936, which was later developed into many other designs. During the Korean War, the United States Air Force used helicopters for transport, observation, and medical evacuation, signifying the advantage helicopters can bring to the battlefield. The first significant use of helicopters in the battlefield in multi-role capacity occurred in the Korean War, with the United States Army deploying many Bell UH-1 Iroquois helicopters for front line missions, including insertion/extraction, supply drops, airborne support, and air ambulance [1]. Figure 1 shows a Bell UH-1 landing in Vietnam picking up troops in a battlefield.

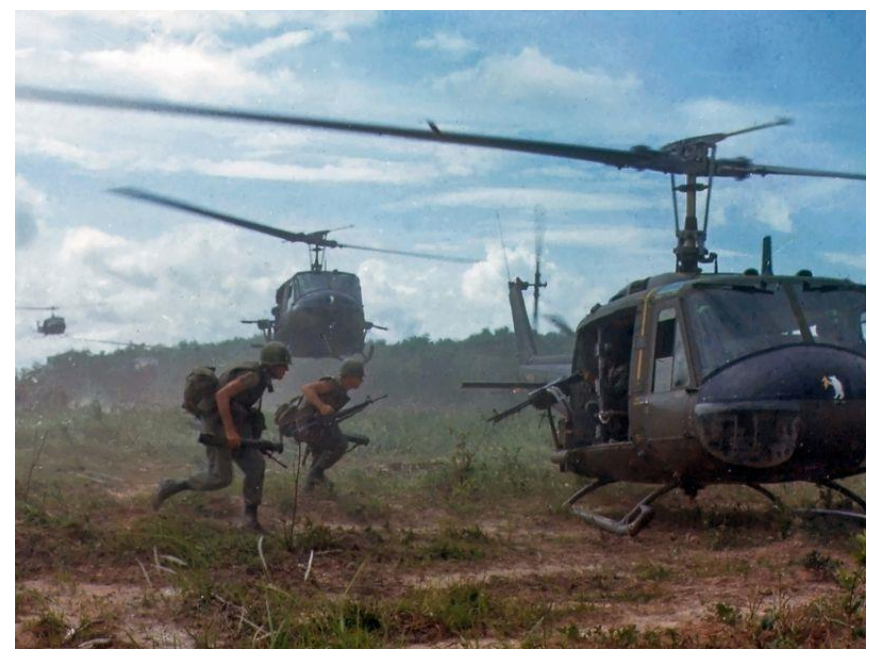

FIGURE 1: A UH-1 HUEY HELICOPTER LANDING IN VIETNAM INTO A BATTLEFIELD [2].

Helicopters were vastly used as they provided ample infiltration and exfiltration capabilities into and out of remote areas and unprepared landing zones. The use of helicopters in unprepared landing zones realized problems such as recirculation, creating brownouts and whiteouts. The primary cause of brownouts and whiteouts in helicopter operations occur in unprepared landing sites due to helicopter downwash [3]. Downwash is the downward flow of air through a rotor disk and is the same as the induced flow through a rotor disk. In hover, downwash is equal to the net upward thrust of the rotors. Air drawn in from above the rotor blades is accelerated to provide lift and thrust, the accelerated flow through the rotors spreads in all directions. Figure 2 illustrates how helicopter downwash propagates and acts in different flight conditions. When the helicopter enters recirculation zone over an unprepared landing site the loose particles of dust, sand or snow is lifted and thrown around near the rotor blades, obstructing the cockpit view of the terrain. While transitioning from forward flight to hover, the downwash on the terrain causes immediate lack of visual information about spatial awareness to the pilot. 
(a) Hover taxi

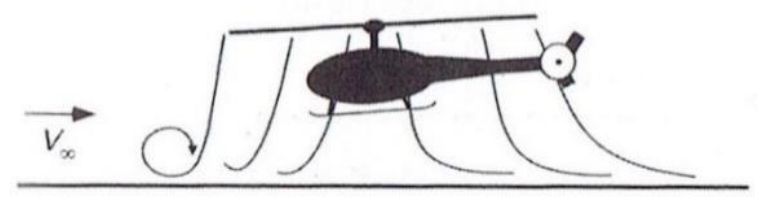

(c) Low speed forward flight

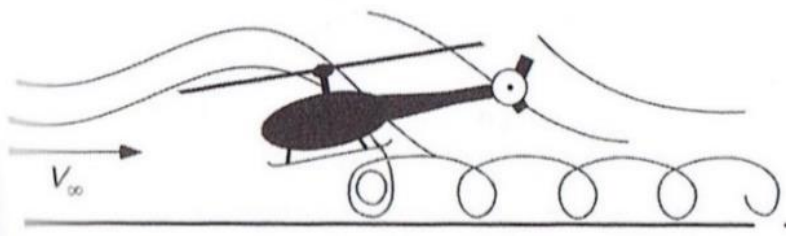

(b) Transition to forward flight

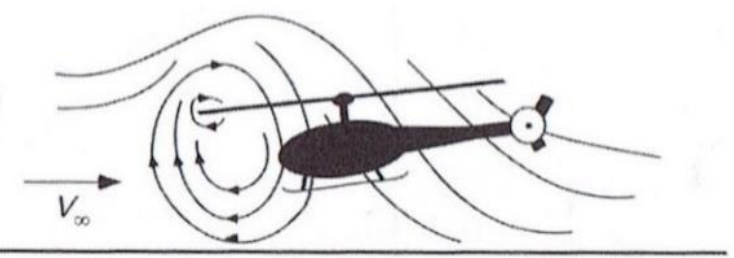

(d) Higher speed forward flight

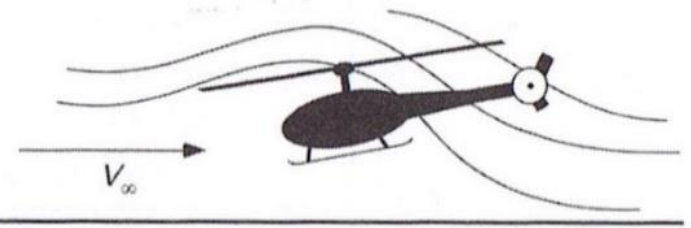

FIGURE 2: ILLUSTRATION OF RECIRCULATION IN HOVER AND FORWARD MOTION, CREATING A DVE SITUATION IN A DUSTY LANDING AREA. (A) IN HOVER TAXI (B) TRANSITION TO FORWARD FLIGHT (C) LOW SPEED FORWARD FLIGHT (D) HIGHER SPEED FORWARD FLIGHT [4].

Degraded Visual Environment (DVE) is a situation in which there is a lack of visual cues from the environment for the pilot, with partial or complete loss of visual cues. Night flights, fog, brownouts/whiteouts are all common causes of DVE [3].

\section{DEGRADED VISUAL ENVIORNMENT (DVE)}

Helicopters have been used in critical roles in proving themselves to be force multipliers in modern-day conflicts in Iraq and Afghanistan. Helicopters have seen significant use in civilian applications, whether it be policing, emergency medical evacuation or media. Emergency medical services, police and military helicopters must be available around the clock in any weather or environmental conditions and their operation cannot be hindered due to DVE or weather anomalies [3].
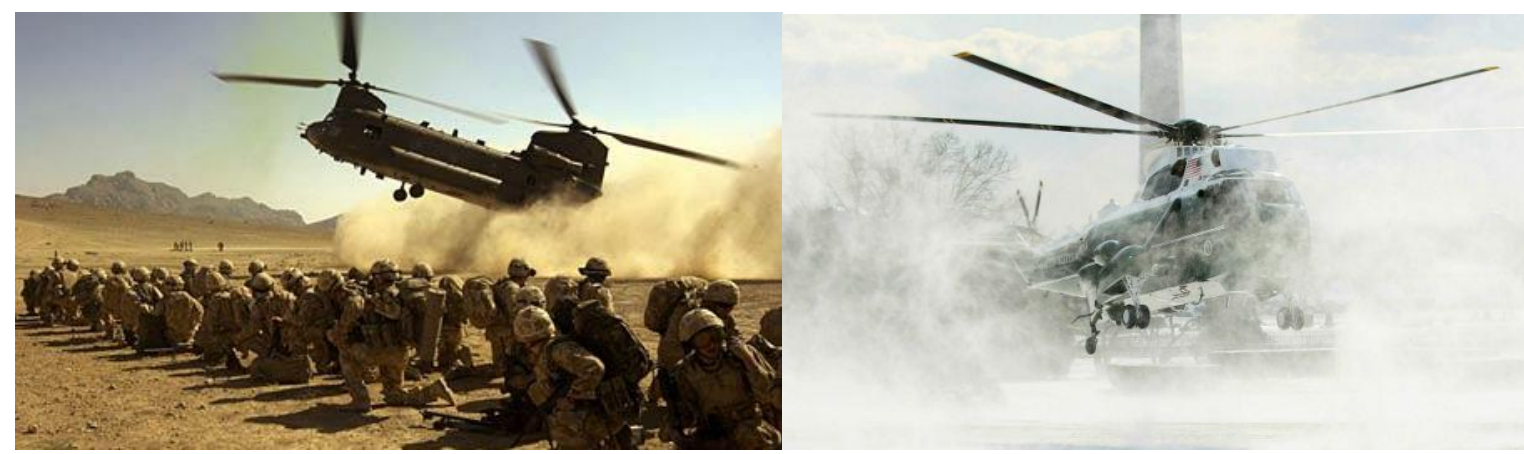

Figure 3: A Figure ILlustrating A BROWNOUT (CH-47 CHINOOK) AND A WHITEOUT (VH-3 SiKORSKY) DUE TO RECIRCULATION EFFECTS [5]. 
With their ability to hover and Vertically Take-Off and Land (VTOL), helicopters see a broader range of operational environments and mission scenarios compared to fixed-wing aircraft. Their ability to perform such dynamic roles, allow them to operate in unprepared and undeveloped areas. Helicopter downwash leads to significant issues for flight safety on unpaved landing zones and other DVE instigating environments. There are several naturally occurring and manufactured DVEs: brownout, whiteout, smog, clouds, night and flat lights. DVE accounts for many crash landings yearly due to operating in DVE situations. As reported in FlightFax by the US Army, brownouts and whiteouts alone account for $24 \%$ of Class A/B flight accidents and resulting in $80 \%$ fatalities between 2002 and 2015 with aircraft losses totaling to \$1 Billion [6]. Class A accidents are damages of property over \$2 million and class B are damages costing between $\$ 500,000$ and $\$ 2$ million suffered by the US Military [6].

\subsection{OBJECTIVE DEFINITION}

Traditionally, helicopter pilots operate at night via the use of a headlight/spotlight, mounted to the front of the aircraft as an accident mitigation method, however, headlights or spotlights are not feasible options in brownouts/whiteouts, foggy, torrential rains and snow [7]. A headlight/spotlight look forward direction at what the pilot is flying at and does not look through dust or snow to build an image. A new solution has been suggested to provide the pilot with increased situational awareness allowing them to operate safely and efficiently. A multi-organization collaboration has been undertaken to solve the helicopter DVE issue at a simulation level to learn and understand the complexities of solving the issue in real-time application. Helicopter pilots are always required to operate in any given condition, putting them in considerable risk, propagated by lack of situational awareness and excessive workload and stress. The National Research Council - Flight Research Laboratory (NRC-FRL), Defense Research Development Canada-Valcartier QC (DRDC-Valcartier), Ryerson University Department of Aerospace Engineering, Queens University, Royal Military College (RMC) of Canada, Les Instrument Optiques du Saint-Laurent inc (IOSL) and CAE, Presagis have collaborated to develop the DVENS system funded by the Consortium for Research and Innovation in Aerospace in Quebec(CRIAQ) to simulate, train pilots and study operations in DVE with such a system. The goal of the DVENS system is to simulate a scenario in which a LiDAR scans an active area to specify a zone to be safe to land on based on pre-existing conditions of the selected helicopter landing capabilities $[7,8]$. In the scenario that DVENS system signifies an area as safe to land over a specified range, the pilot can select a zone by touch capability, where $3 \mathrm{D}$ world conformal symbology would be displayed to help them land providing virtual cues for situational awareness. In the orthographic plane of the Head Down Display (HDD) the pilot is presented with helicopter performance, navigation, and control information to build a mental understanding of the helicopter body's relationship with the operating environment [9]. The combination of the LiDAR and symbology provide much needed and improved situational awareness for the pilot in DVE operations. The simulation allows for critical objectives to be set 
forward for the implementation of the technology towards helicopters, providing the ability to understand capabilities and resolve potential issues. Ryerson Aerospace department's objective in this project is the development of 2D, 3D symbology, obstacle, and terrain representation as a subject of Human Factors and Human-Machine Interfacing (HMI) study. Ryerson has been extensively advised by NRC FRL's research officers in the implementation of the symbology and terrain textures to help improve pilot perception. The LiDAR simulated for the project is a Neptec 3D Obscurant Penetration Asynchronous LiDAR (OPAL) with an increased scanning capability due to its Double Risley Pair (DRP) architecture. This project provides a fusion of incoming LiDAR data and updates it in real-time with pre-loaded Digital Elevation Map (DEM) on the backend level with a symbology displayed in the front end. Backend level consists of the logic and algorithms which drive the simulation. The front-end level consists of the representation of the graphics and display, which will be utilized by the pilot. An initial study performed at DRDC through the Multipurpose Airborne 3D Polarimetric Imaging Radar Assessment (MATPILA) project explored the possibility of integrating a see-through OPAL LiDAR with Synthetic Vision Systems (SVS) to help pilots in operating in brownouts [10]. The Double Risley Prism (DRP) LiDAR simulation was utilized for the system and is used for 3D mapping applications. Single pair Risley Prism is robust with the continuous operation; however, DRP offers scanning of a larger FOV and increased sampling point density and sampling uniformity (Enhanced Scanning agility using a second pair of Risley Prisms). The DRP architecture is part of an OPAL which is capable of penetrating dust clouds, fog, rain particles to see through it and build a point cloud image[10, 11].

\subsection{RESEARCH TOOLS: CAE, PRESAGIS SOFTWARE PACKAGE}

In the developments undertaken, Commercial-Of-the-Shelf (COTS) simulation tools were used to develop and evaluate the symbology. Presagis is a subsidiary of CAE simulation-based in Montreal, Canada. Presagis provides the COTS simulation, graphics software and services for defense and aeronautics organizations worldwide. The software suite provides rapid development high fidelity and scenario, environment management through objected-oriented programming via $\mathrm{C}++$. The architecture of how the software works together is provided in Figure 5.

\section{PRESAGIS HELI CRAFT}

The CAE, Presagis HELI CRAFT (shown in Figure 4) is a high-fidelity customizable simulator developed with the latest Presagis Modelling and Simulation (M\&S) products. HELI CRAFT is an adaptable/modifiable system with a dedicated development environment package through Software Development Kits (SDKs) for each Presagis software suite product. Capabilities of HELI CRAFT allows for modification and customization, which is ideal for a research environment. The HELI CRAFT features are three major components, (1) Helicopter Simulation, (2) Environment Simulation and (3) Cueing 
simulation along with an Instructor Operating Station (IOS) for enhanced control, management, and training on the system. The IOS can externally control all the simulation parameters. HELI CRAFT's helicopter simulation component is powered by Presagis, CAE HeliSIM. HeliSIM is a high-fidelity flight simulation tool that provides dynamic real-time simulations of rotary-wing aircraft. The platform consists the Flight Dynamic Models (FDM) of numerous helicopter systems, and the BELL 412 is used in the context of the DVENS project [12]. The FDMs are modifiable, and the software also allows for high fidelity and rapid development to change environment phenomenon, navigation parameters, etc. The HELI CRAFT system is used extensively for the research to study the motion, the pilot workload in operation to land in DVE.

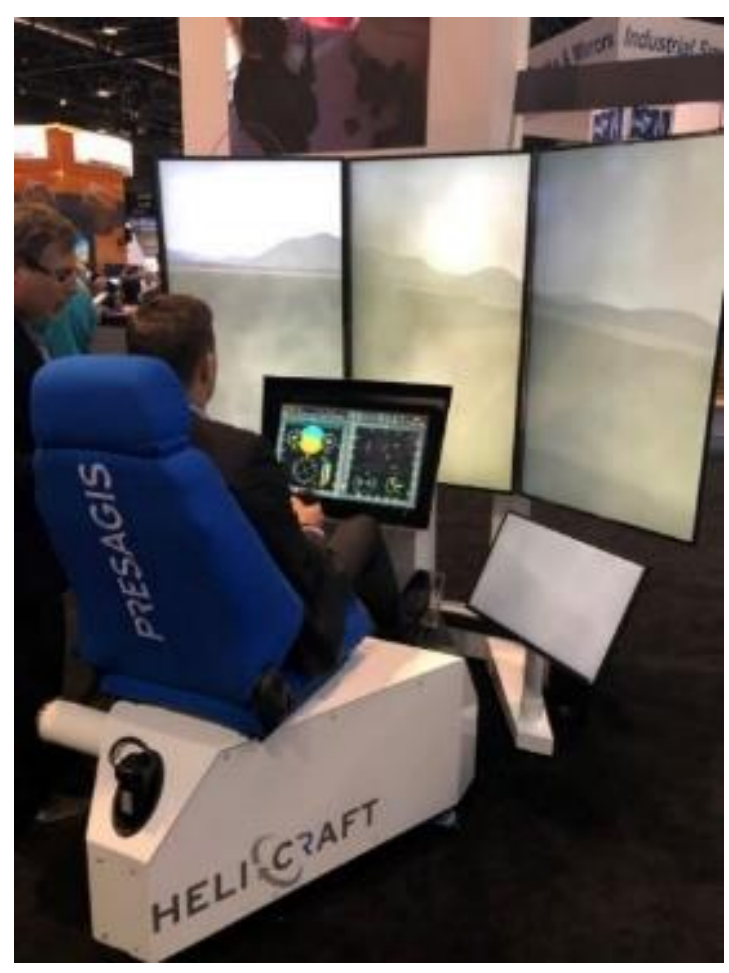

Figure 4: THE CAE, PRESAgis HELI CRAFT HELICOPTER SiMUlATOR [13].

\section{PRESAGIS VEGA PRIME}

Vega Prime provides high-quality visualization and graphics for the representation of the environment in any scenario[14]. In the case of DVE, Vega Prime provides the Out-of-the-Window (OTW) cockpit view and the implementation of a Dust Cloud representation. Vega Prime is used to display the HDD symbology for pilots to land with virtual VFR cues. Vega Prime provides the necessary means to implement the 3D cueing symbology that is developed as part of this thesis via OpenGL API. 


\section{PRESAGIS VAPS XT}

Presagis, CAE VAPS XT is a high-fidelity Human-Machine Interfacing (HMI) and embedded systems tool that is utilized to generate flight displays that are shown on Heads Up Displays (HUDs), Head Down Display (HDD), Head Mounted Displays(HMDs) [15]. The flight performance, control, navigation information displays are designed on VAPS XT.

\section{PRESAGIS ONDULUS IR AND LiDAR SIMULATOR}

Presagis, CAE's Ondulus IR is a physics-based Light Detection and Ranging (LiDAR) simulation system, with the ability to simulate a range of Radar and LiDAR systems. Ondulus IR accounts for radiation, cooling, conduction, convection and evaporation and an array of environmental scenarios and atmospheric conditions. Ondulus IR accounts for energy exchanged between the sun and a range of materials (Concrete, sand, buildings, insulation, walls, etc.) $[16,17]$. Thus, via the Ondulus IR software framework, a DRP based OPAL Lidar is simulated for looking though the dust or snow.

\section{NCOM COMMUNICATION PROTOCOL}

HeliSim and VAPS XT can be linked between each other and among other Presagis software through intercomputer communications operating on a single network. Communications are setup via $\mathrm{C}++$ programming with data transmission occurring over low-level Transmission Control Protocol (TCP) or User Datagram Protocol (UDP). A parallel connection can be set up on multiple computers to allow multi-level communications and data sharing [15]. Thus, simulation mathematics can be run in the back end, while visual simulations can be run on a different PC.

\section{OPEN GL AND GIMP}

Open Graphics Library (OpenGL) is a C++ based Application Programming Interface (API) utilized to render 2D and 3D vector graphics [18]. The API uses a computer's Graphics Processing Unit (GPU) to create advanced rendering concepts. The OpenGL concept is extensively used in the study as the designed 3D symbology and VAPS XT from CAE, Presagis is based on the API [15]3. The graphics are pre-existing for VAPS XT and Vega Prime, with customization capabilities for the addition of new features such as 3D symbology.

For texture development the open-source graphics editing software GIMP 2.8 is used to create textures that would represent the terrain curvature. The software is capable of editing and creating textures patterns that effectively provide a better representation of the terrain. Developed or edited textures are exported as .rbg format and placed into the Vega Prime architecture to define and overlay the existing CDB and terrain data. 


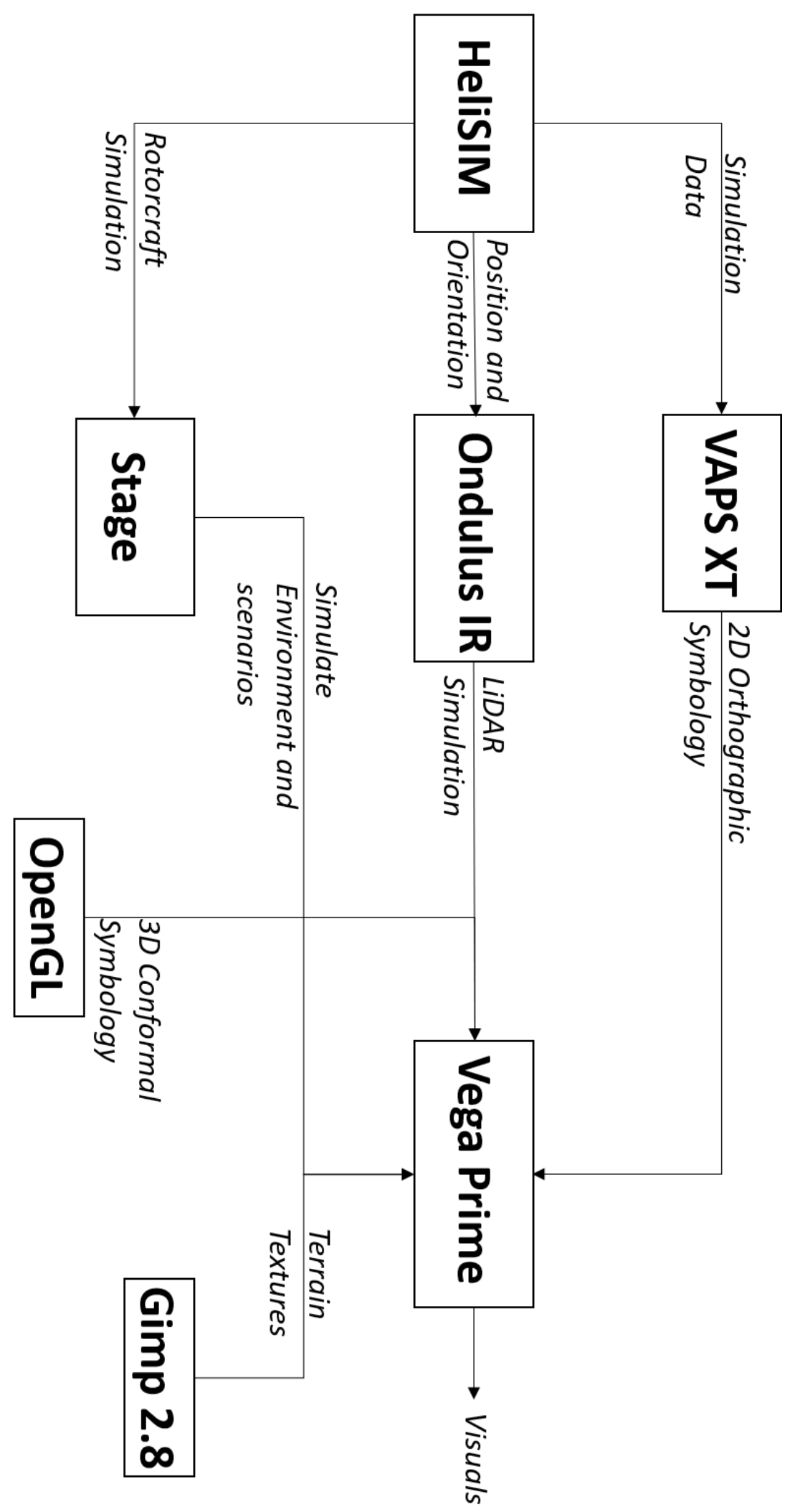

FIGURE 5: MAP OF SOFTWARE TOOL INTEGRATION. 


\section{CHAPTER 2.0 LITERATURE REVIEW}

\subsection{PROBLEM-SPECIFIC STUDY}

Rotorcraft flight in DVE has been a long-standing issue. In 2006 the National Transportation Safety Board (NTSB) of the United States reported increased accidents due to DVE flying and recommended utilizing Terrain Awareness and Warning Systems (TAWS) and other technologies to mitigate this trend. The Netherlands Aerospace Centre (NLR) has worked alongside the European Helicopter Safety Team (EHEST) to reduce accident rates and explored almost 145 technological solutions to mitigate DVE related accidents [19]. According to a report from 2014, enhanced TAWS and obstacle detection systems for helicopter lowlevel flight guidance aids are ranked as the top possible solutions for enhanced safety. Technological solutions can be used to increase pilot situational awareness, helping them understand the external environment to help improve pilot perception, judgment, and actions, this will help to reduce obstacle collisions in low visibility conditions. North Atlantic Treaty Organization (NATO) Industrial Advisory Group (NIAG) determined three possible development areas of further advancements to solve the DVE issue for flight in low altitude [8]. Figure 6 shows the suggested working areas by NATO NIAG to mitigate the DVE situation and help pilots operate in DVE. Figure 7 shows the fatalities suffered by the United States Armed Forces due to non-combat losses, where a majority of the reasons of emanate from the calamities caused by situation similar to DVE.

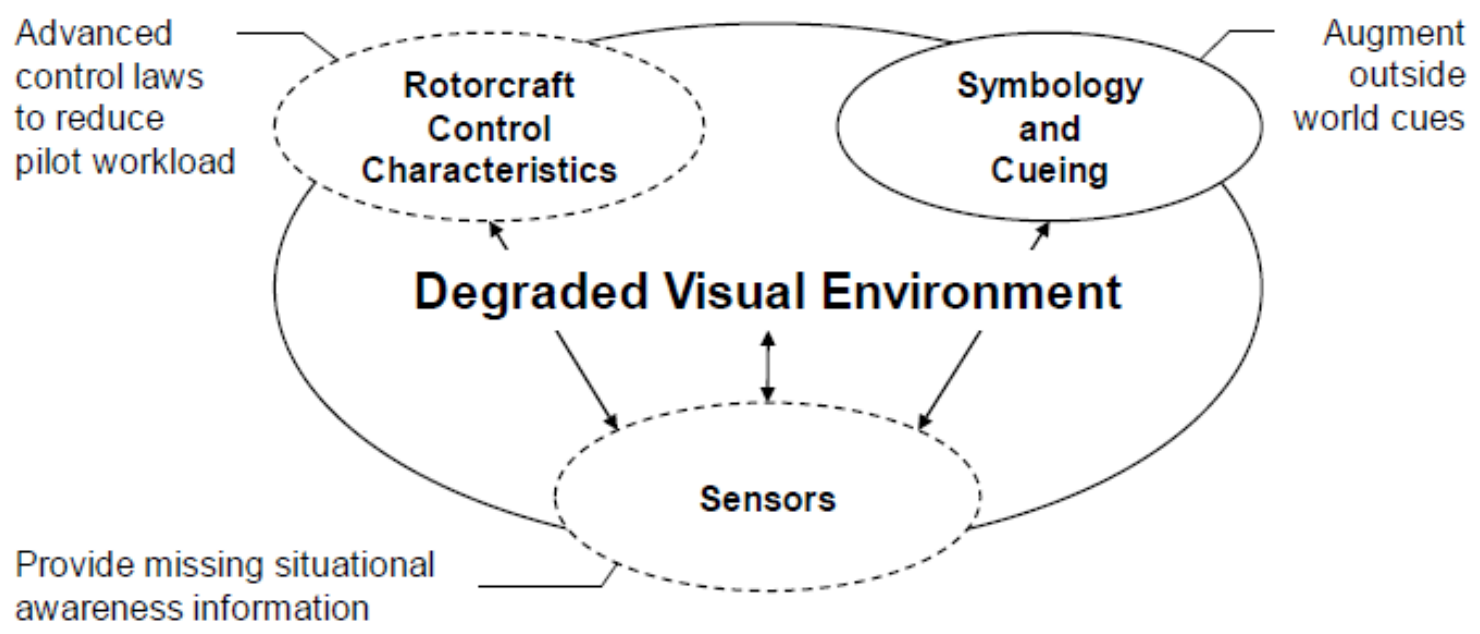

FIGURE 6: NATO SUGGESTED WORKING AREAS FOR SOLUTION TO SOLVING DVE ISSUES[8]. 


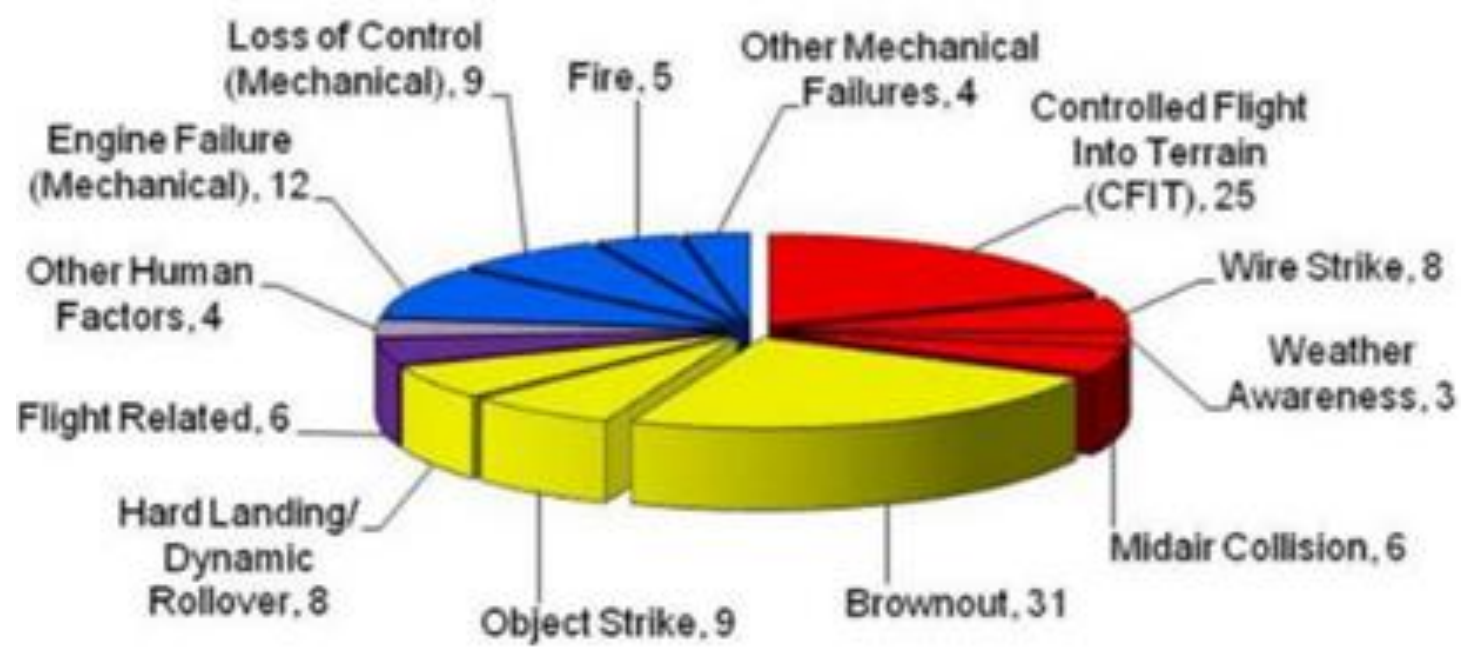

FIGURE 7: REPRESENTATION OF ROTARY WING NON-HOSTILE LOSSES (130 EVENTS) [9].

\subsection{SITUATIONAL AWARENESS AND ILLUSIONS IN AVIATION}

When piloting rotorcraft, the pilot is operating in a 6 degree of freedom environment. SA is defined as "Perception of the elements in the environment within a volume of time and space, comprehension of their meaning and projection of their status in the near future."[20]. In-flight, a pilot is exposed to three forms of Situational Awareness (SA); spatial awareness, system awareness, and task awareness[20, 21]. These are described as;

1. Spatial awareness is the position in which a pilot must be aware of the environmental and flight instrumentation [21].

2. System awareness is the case in which a pilot is kept aware of actions being performed by automated systems for upkeep of attention towards task management [21].

3. Task Awareness is a situation in which a pilot must maintain consistent awareness of the objectives of a mission at hand [21].

Workload consisting of spatial, system, and task awareness requires considerable mental capabilities [21]. SA is a crucial component of any flight scenario and can be broken into a three-stage idea, cognition, and working memory, relevance to dynamic and evolving situations, and maintaining distinctiveness in operational scenarios. Cognition and working memory are temporary information stores, which are forgotten if not rehearsed. Muscle memory is opposite to working and cognition and is engrained due to extensive practice and repetition. Working memory and cognition are relevant to dynamic situations, where a pilot observes and understands evolving situations, unlike long-term memory. A situationally aware pilot can build a mental representation of a changing environment and understand an evolving situation by combining existing information in their cognitive memory [21]. 
A situationally aware pilot can operate in an unknown environment through an unexpected scenario. Spatial awareness is a critical factor which defines the aircraft moving through 3D space populated by hazards. Flying in a DVE, the pilot must uphold numerous cognitive tasks, the motion deals with six variables that are to be monitored and controlled. The six variables are three angular orientations of yaw, roll and pitch, and three-position variables altitude, lateral deviation, and position on the designated flight path. All the six variables are also cross-coupled in flight dynamics, in which changes in the orientation variables determine the future position changes. A pilot operating in DVE must be cognizant of the orientation and position of the helicopter and spatially aware of the operating environment, which is not possible when the terrain is not visible during DVE [1].

The extent of spatial information pilots utilizes to perform landings is essential given their abilities. In new locations unfamiliar to the pilot, they completely rely on visual cues to operate. Pilots rely vastly on the terrain information to position themselves about the operating environment. To completely understand the extent of the information use models of information processing and decision-making need to be explored $[22,23]$.

Processing of information in the human brain involves the transmission of signals from the sensory system via neural pathways; the processed information is interpreted in the brain leading to decision making. Existing weak links in the process can lead to inappropriate decisions and actions. Weak links can be lack of information itself. A complete situational awareness model would look to have a spatial understanding of the terrain and the helicopter's relation to the terrain. Flight information alone cannot provide the complete picture; spatial awareness is also essential. Information sensed from the terrain or environment can be misleading via illusions perceived from the visual or vestibular systems [20, 23].

Illusions are experienced based on what the eyes see, which can perceive wrong information depending on time, location, and operating conditions. Vision is divided into two channels and provides important sensory information to the pilot during flight. Central or foveal vision helps the pilot identify objects and perception of colors. Peripheral or ambient vision is involved with the perception of movement and provides peripheral cues to maintain spatial orientation. The brain uses information attained from vision to make a judgment regarding the distance, speed and relative movement of objects $[1,20]$. The following cues are used by the visual system:

\section{Comparative size and shape of known objects at different distances}

2. The relative velocity of images moving across the retina. Objects that are close are perceived as moving faster than more distant objects. 
3. Interposition or layering of known objects. If one object is placed in front of another, it is perceived as being closer.

4. Texture and contrast of known objects, cues lost as objects move further away.

5. Changes in the illumination of objects due to light and shadows.

Vision provides a reliable source of information. In some circumstances, however, external factors can influence vision to interpret information contrary to reality. Illusions can confuse the brain into processing information that is entirely opposite to reality, causing a lapse in judgment[1]. Some common illusions in aviation are;

1. Up-sloping terrain/runways: can produce the illusion of too-high of an approach, the pilot may respond by lowering the nose to decrease altitude and can run into the ground if performed at low altitude $[1,20]$.

2. Down-sloping terrain/runways: can produce an illusion of too-low an approach, the pilot may respond by raising the nose of the aircraft to increase altitude which can lead to a stall or landing further down the runway.

3. Black-hole illusion: Occurs during final approach over water or at night or unlit terrain when the horizon is not visible beyond the lighted terrain. Lack of peripheral cues makes it difficult for pilots to orient themselves with the earth $[1,20]$.

4. Autokinetic Illusion: Gives pilot the impression that a stationary object is moving in front of the path of the aircraft, it is caused by the pilot staring at a single stationary point of light in an otherwise featureless background. Pilots are susceptible to perceive up to 20 degrees of movement in any direction in succession even though the object is fixed with no displacement [20].

5. Vection Illusion: A sensation of moving backward when a vehicle next to the pilot is moving forward.[24]

Most helicopter operations occur via Instrument Flight Rules (IFR) with assistance from air-traffic controllers and radar, however, in unfamiliar terrain, Visual Flight Rules (VFR) prove to be an important visual cue for pilots to detect and avoid obstacles. The visual flight phase conducted are very demanding and hazardous to pilots; the addition of DVE further increase the risk involved with VFR based flying. Combined research conducted between the Federal Aviation Authority (FAA) and the U.S. Army conducted flight-test to study rotorcraft flying qualities in DVE with degraded but not zero visual cueing [22]. The study found the pilot workload to maintain rotorcraft control to be very high, which left the pilot over-worked to maintain an awareness of the rotorcraft position, orientation, and flight concerning 
obstructions or the ground. This lack of situational awareness could lead to a catastrophe. Results from human factor experiments indicate the combined effects of DVE, turbulence, and basic rotorcraft handling amounted to $100 \%$ of the pilot's workload capability [22]. The NTSB found the loss of situational awareness to correlate with many of the accidents, where even experienced pilots made mistakes in flight when encountering a DVE. The NTSB found that pilots tend to drift into objects within their field-of-view, inadvertently transitioned their aircraft into side or rearward drift flights and flew into the ground. Visual conditions for control of rotorcraft for civilian operations is defined via Instrument and Visual Meteorological Conditions (IMC and VMC), which define if a pilot is flying the aircraft via looking inside or outside the cockpit. Both IMC (presently embedded flight decks) and VMC methods do not provide significant traction in being aware of rotorcraft flight in a DVE with obstacles that may lie around the aircraft [22]. One of the primary visual cues required to stabilize a rotorcraft hover and lowspeed flight is using "microtexture" (natural indicators, such as grass, small stones, etc.) [8, 20, 22] which would exist in the pilot's Field-Of-View (FOV) is obscured in DVE conditions (snow, darkness,etc.). Experiments developed in this study suggested that flight in an un-augmented helicopter account for $70 \%$ of the pilot's workload capacity, leaving only $30 \%$ to maintain any situational awareness[22]. Other factors that would require attention from the pilots are spared no attention proving leading to a lapse of attention and a stressful environment. External effects can further instigate the stress and workloads levels of a pilot, such as turbulence or high winds, obstacle (static and dynamic). Figure 8 highlights the extent to which a DVE can engulf a pilot's SA.

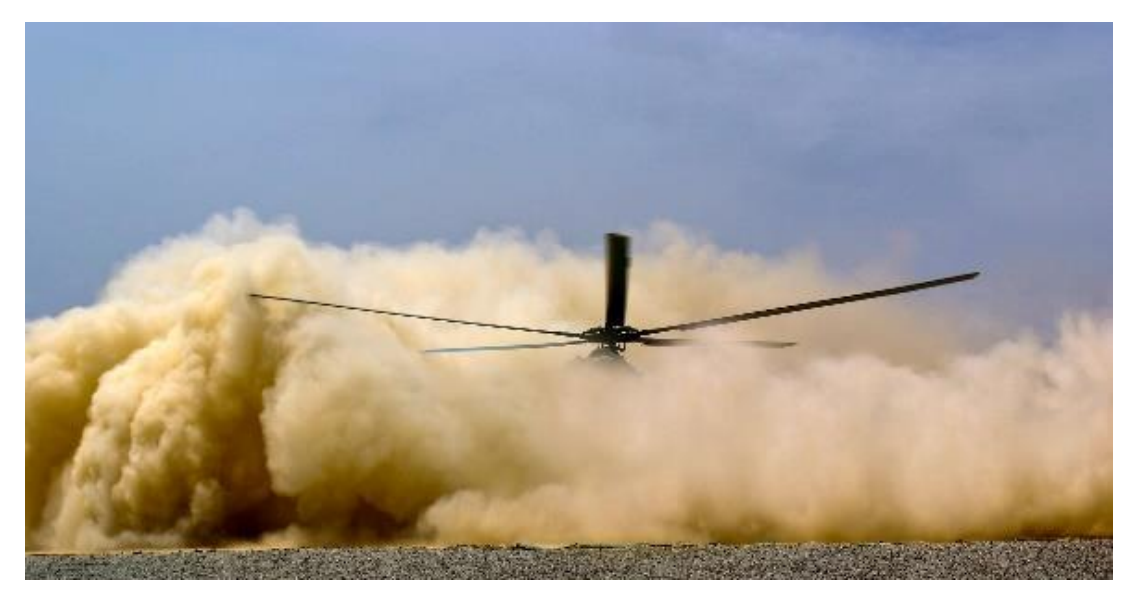

FIGURE 8: A HELICOPTER COMPLETELY IMMERSED IN A DUST CLOUD [25].

Thus, a pilot operating in DVE is subject to a high workload with minimal terrain cues in the approach and hover phases and barely any awareness while close to landing. In this critical situation, the pilot must be able to identify a Safe Landing Zone (SLZ), obtain a clear understanding of the obstacles around the SLZ, be aware of the flight performance characteristics along with the minimizing the drift characteristics of aircraft in DVE. These actions and duties must be performed while the pilot considers the navigation, 
control, and performance of the helicopter. In the case of a military operation in a hostile zone where there are threats present that will harm the helicopter, the workload, stress will increase tremendously. Thus, enhanced vision systems that maximize pilot understanding of the situation via spatial, system, and task is an immense asset proving safer operations.

\section{INFORMATION PROCESSING AND DECISION MAKING}

Human reaction to scenarios is different for everyone, but the basic model of information processing is used to describe the decision making for everyone. If the model of information processing and decision making is the same, the outcome should be predictable. However, the reactions to the information and decision taken are different from human to human. Pilots are generally trained to operate similarly throughout with strict standards and Standard Operating Procedures (SOPs). Thus, a single information processing and decision-making model can suffice for pilots in general. In an aerospace environment, there are six major human error perspectives;[21] Cognitive, ergonomic, behavioral, aeromedical, psychosocial, and organizational perspectives. Of all the perspectives the single most influencing for the DVE scenario under investigation is the cognitive perspective; studying how pilots process mental information, attention, language, memory perception, problem-solving, creativity, and thinking. The ergonomic, behavioral, aeromedical, psychosocial, and organizational perspectives are all large-scale topics whose subsets are branched in a manner that cannot be improved via the study of human factors alone.

When pilots' loose cues to what they see outside, and fly based on experience or available situational awareness, they are taught to think and act similarly via training. The introduction of new virtual cues can act to provide the necessary situational awareness that would rather be unavailable in DVE. The objective is to provide the pilot with a visual means of cueing and to not optimize their psychosocial (interrelation of social factors and individual thought and behavior), ergonomic or personal level aeromedical situation. A cognitive perspective provides the lead by generalizing the human information processing system to understand how people think and react. The cognitive perspective suggests that information from the environment is sensed through one of the senses (taste, sight, touch, smell, and sound) upon which stages or mental operations response is generated[21]. Figure 9 and 10 show the information processing and decision-making models respectively which highlight the cognitive process described above. 


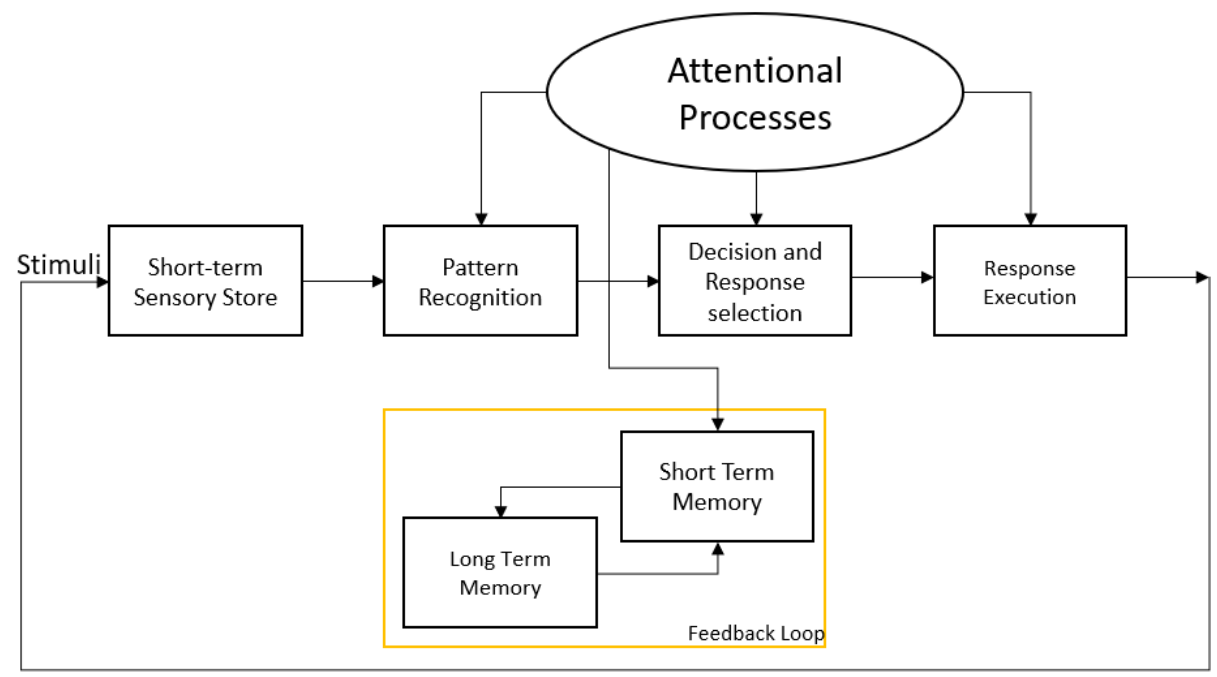

FIGURE 9: THE BASIC MODEL OF INFORMATION PROCESSING ADAPTED FROM WICKEN AND FLACH (1988) [21].

According to Wickens and Flach (1988), their four-stage model for human information processing describes stimuli from the environment are transmitted as neural impulses and temporarily stored as short-term memory. The short-term is compared to previous patterns in the long-term memory to build a mental representation concerning the present state. Once the representation is built the individual would execute a response, which can be ignored or acted upon [21].

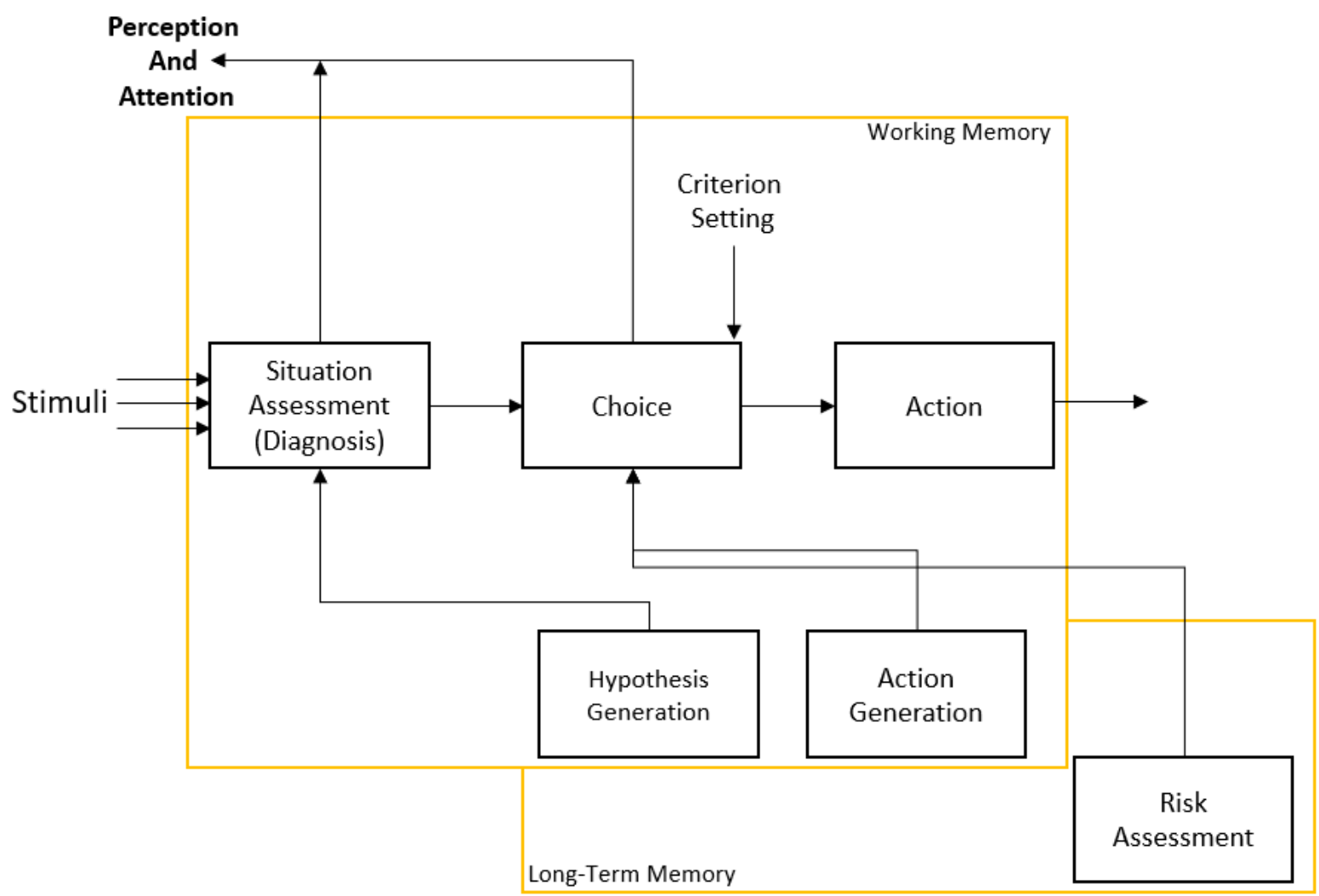

FIGURE 10: DECISION MAKING MODEL ADAPTED FROM WICKENS AND FLACH (1988)[21]. 
Wickens and Flach also suggested the Decision-making model, which suggests that pilots would sample numerous cues in the environment to assess a situation. The cues attained are then compared to pre-existing knowledge from training and mission briefings. This approach requires an evaluation of possible actions, risk assessment, and goal setting to ensure an appropriate response. Pilots at this point look for cues that would supplement their decision-making ability by increasing their perception and attention which can improve their situational awareness and enhance response [21].

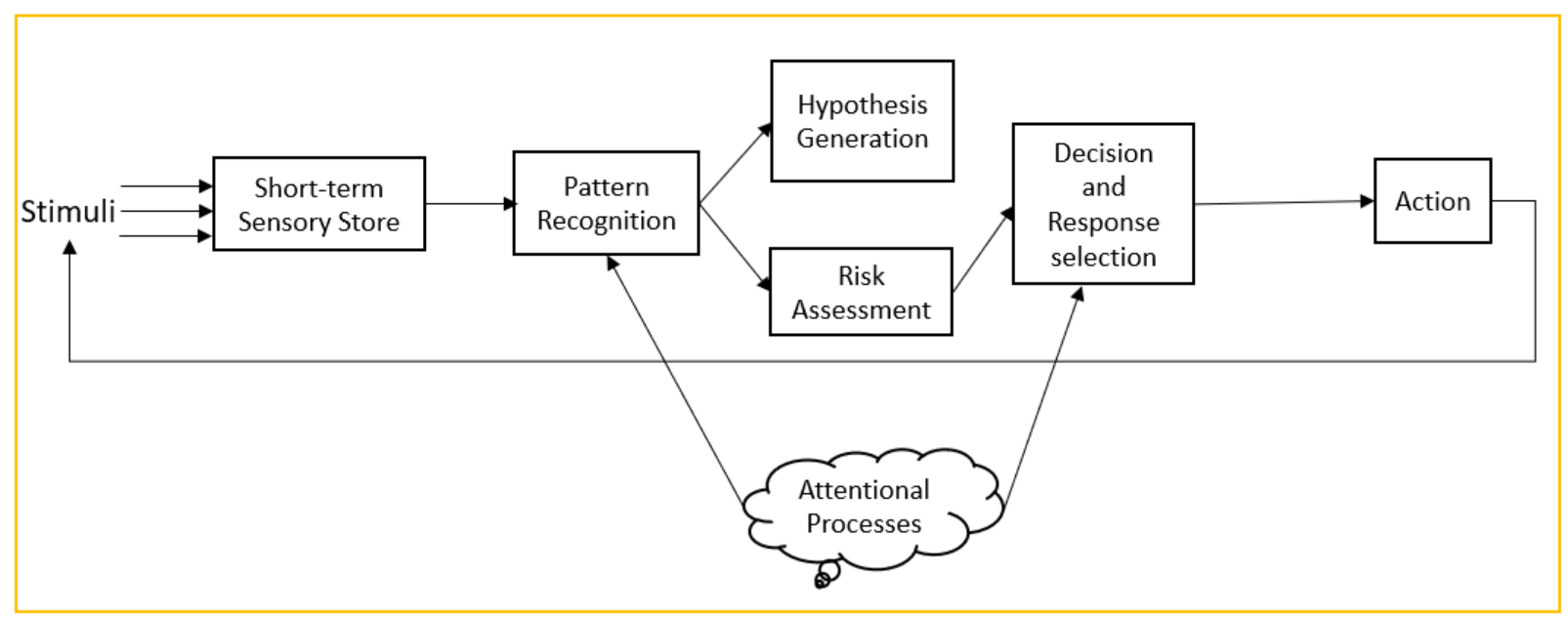

FIGURE 11: COMBINED INFORMATION PROCESSING AND DECISION-MAKING MODEL.

In the case of DVE flight in hover, the pilot losses visual cues OTW and only rely on flight instrumentation to understand their orientation and motion. The only reference of the environment they might have can come from long/short term memory. Long term memory can serve by building a mental representation of what the pilot remembers while making the approach and terrain information that may have been provided in the mission briefing. Thus, the information they remember from before entering a DVE condition is relied on to perform the landing. There is a lack of information when performing a critical landing task.

In 1982 Rasmussen developed a comprehensive and aviation crew centric algorithm (shown in Figure 12) which classifies the resulting information processing errors that may arise in the information and decisionmaking process. The resulting algorithm was a six-step indication to diagnose at what point of the combined information and decision-making processing the aircrew could have failed cognitively. The six-stage diagnostic process consists of stimulus detection, system diagnosis, goal setting, strategy selection, procedure adoption, and actions stages. A fault in any which point or together can be lead to cognitive failure. The derived model adapted by O'Hare et al. called the "A taxonomic framework for assessing aircrew error" provides a capability to not only analyze an accident incident occurring but also to scope why it has occurred in a complex environment. This taxonomy can be applied to any accident analysis to pursue the cause of an unforeseen event that may have been caused by a pilot [21]. 


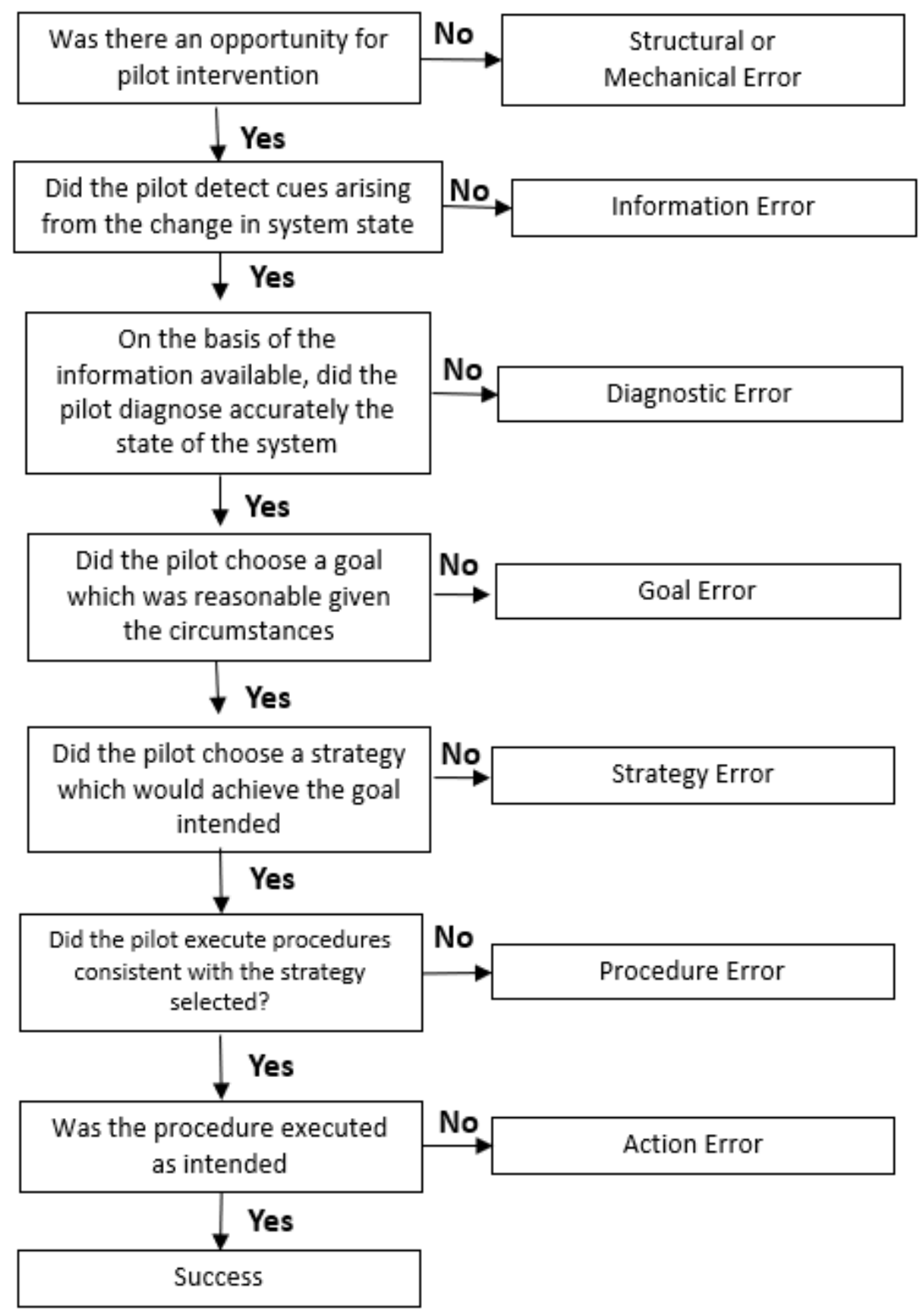

FIGURE 12:THE TAXONOMIC FRAMEWORK FOR ASSESSING AIRCREW ERROR ADAPTED FROM O'HARE ET AL. (1994)[21].

This taxonomic tool is further explored in Chapter 2.4 to provide developmental goals, for error mitigation. 


\subsection{HELICOPTER OPERATIONS AND MANEUVERS}

Helicopter pilots use several methods to perform landings. Landing techniques are briefly described in this section to highlight how quick and intense DVE conditions form around a helicopter to also provide the complexity of operations.

Rapid deceleration or Quick Stop is a method used to decelerate from forward to hover flight. This method is often used to stop during takeoff/landing if something blocks the helicopter path [26]. Figure 13 represents the landing sequence of a rapid deceleration or quick stop maneuver.

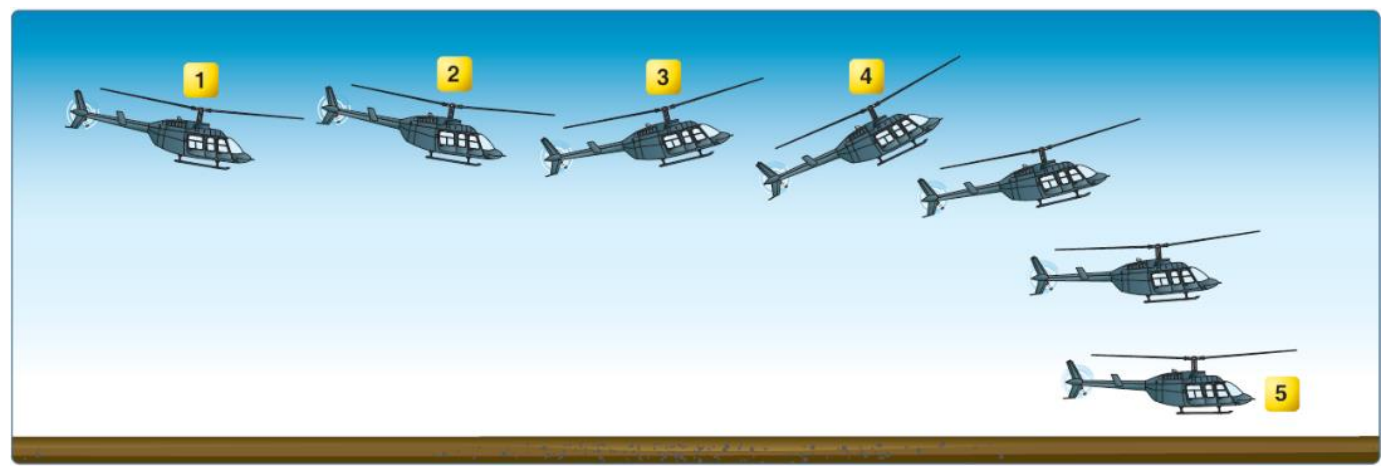

FIGURE 13: A RAPID DECELERATION OR QUICK STOP LANDING DEPICTION [26].

Another method of landing is the steep approach, which is primarily used when there are obstacles in the usual approach path. A steep approach allows entry/landing into most confined areas. Figure 14 represents the landing sequence of a steep approach.

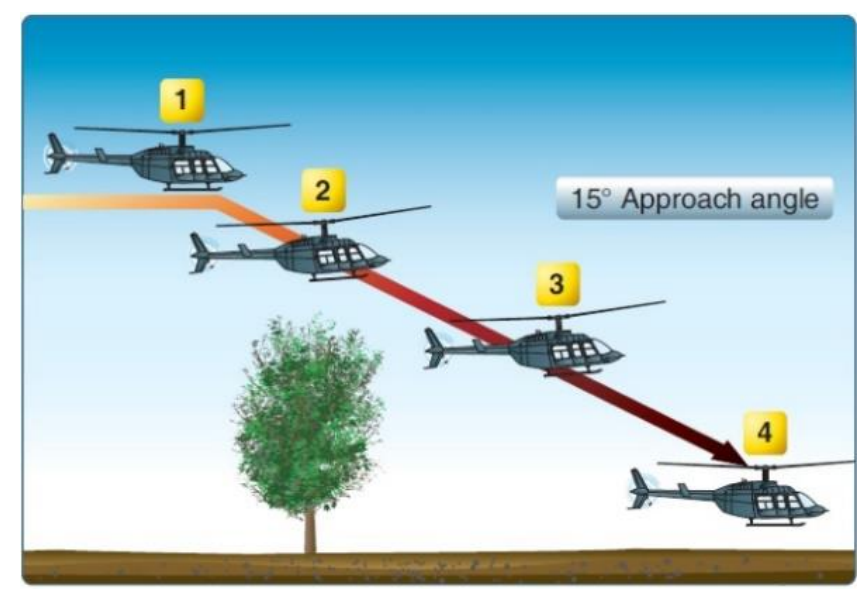

FIGURE 14: STEEP APPROACH TO HOVER METHOD DEPICTION [26].

The third method of landing is the shallow approach and roll-on landing, which uses a shallow approach and running performed in high-density altitude, or at high gross weight conditions, where a steep approach 
cannot be made due to lack of available power to maintain a hover [26]. Figure 15 represents the shallow approach or running landing sequence.

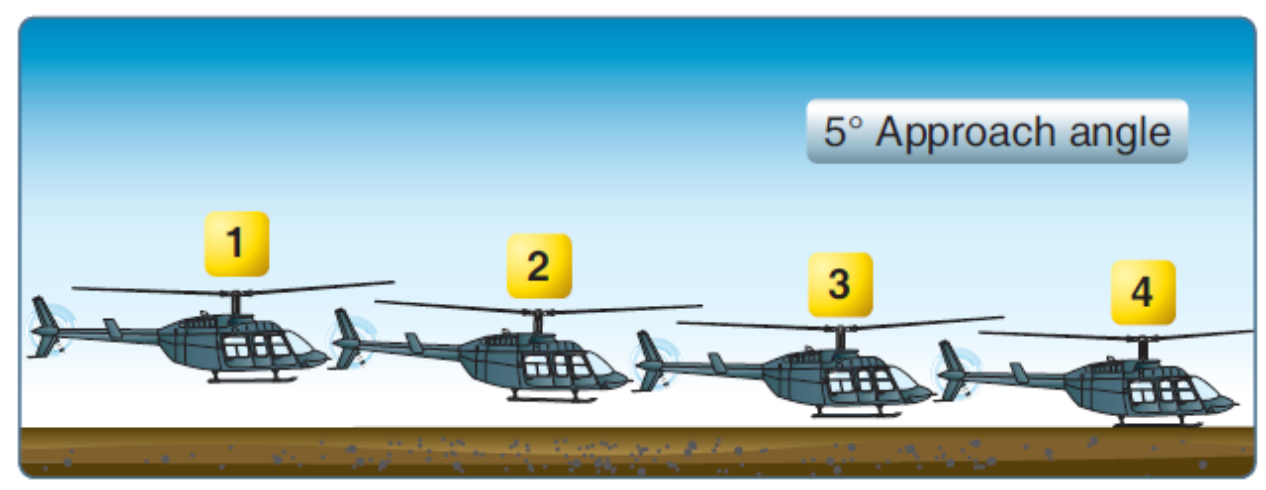

FIGURE 15: SHALLOW APPROACH AND RUNNING LANDING [26].

Landing in unprepared areas can often have slopes of various magnitudes; dynamic rollover is a common accident associated with landing on sloped surfaces if not performed correctly. In the cases of more significant slope operations, one natural phenomenon that can occur is the tendency for slopes to obstruct wind passage, which can cause turbulence. Landing a helicopter against the slope is a recommended practice as opposed to with the slope since there is a chance for the rotor striking the tail boom of the helicopter.
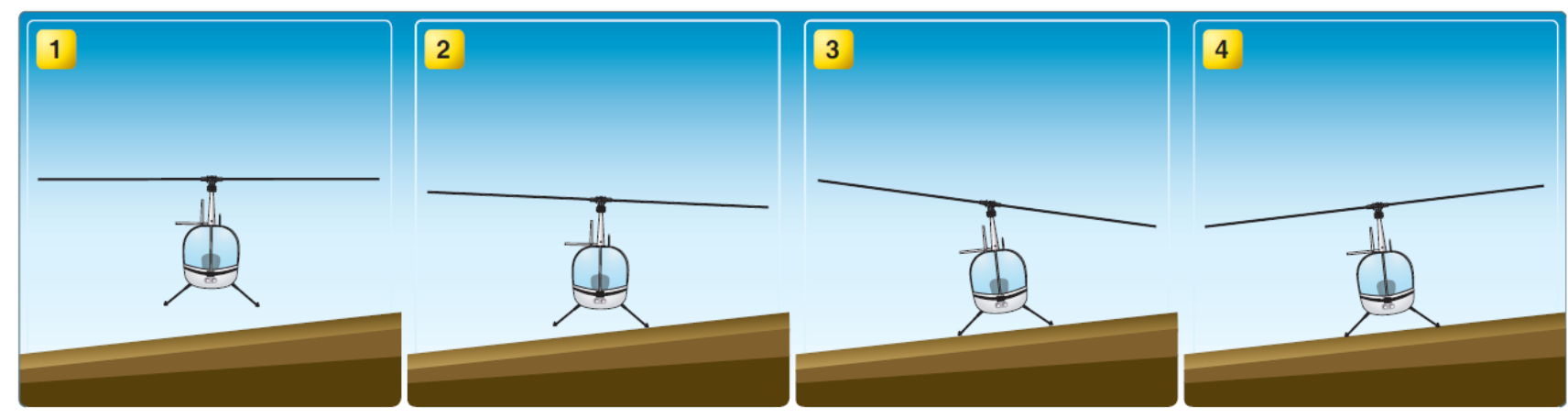

FIGURE 16: DEPICTION OF A SLOPE LANDING [26].

Landing/Takeoff in confined areas can be highly limited due to terrain variation, obstacles placement, allterrain irregularities (slopes, ditches) and confined areas (woods, tree-stumps, wires, poles, buildings) can all add excess duties to a pilot. The helicopter pilot would have to assume the roles of a surveyor, engineer, and manager when selecting this area to conduct the landing. Landing in confined areas especially mentally demanding as the pilot might have to look and think about clearances of the tail rotor from striking any objects in the operating vicinity. Simple objects such as electrical wires, lamp posts are enough to jeopardize a mission. 
Table 1 summarizes a list of possible error sources in landing via different conditions, a pilot unaware of the helicopter's control characteristics concerning the terrain can cause catastrophic errors.

TABLE 1: SUMMARY OF COMMON ERRORS ASSOCIATED WITH THE DIFFERENT LANDING METHODS [26].

\begin{tabular}{|c|c|}
\hline Landing Procedure & Common Errors \\
\hline $\begin{array}{l}\text { Rapid Deceleration and } \\
\text { quick stop (Figure 13) }\end{array}$ & $\begin{array}{l}\text { 1. Failing to effectively control the rate of deceleration. } \\
\text { 2. Allowing the helicopter to stop forward motion in a tail-low attitude. } \\
\text { 3. Using excessive nose high attitudes } \\
\text { 4. Using anti-torque pedals improperly, causing erratic heading changes. } \\
\text { 5. Using excessive nose-high attitudes. }\end{array}$ \\
\hline $\begin{array}{l}\text { Steep Approach } \\
\text { to hover (Figure 14) }\end{array}$ & $\begin{array}{l}\text { 1. Failing to make anti-torque pedal corrections to compensate for } \\
\text { collective pitch changes. } \\
\text { 2. Slowing airspeed excessively to maintain the proper angle of descent. } \\
\text { 3. Failing to achieve hovering altitude, attitude and zero groundspeed } \\
\text { simultaneously. } \\
\text { 4. Using too much aft cyclic to cause rotor strike. } \\
\text { 5. Failure to align landing gear with the direction of travel. }\end{array}$ \\
\hline $\begin{array}{l}\text { Shallow Approach and } \\
\text { running landing } \\
\text { (Figure 15) }\end{array}$ & $\begin{array}{l}\text { 1. Assuming too much nose high attitude to slow helicopter near the } \\
\text { surface. } \\
\text { 2. Failure to maintain heading resulting in a turning or pivoting motion. } \\
\text { 3. Failure to maintain a speed that takes advantage of an effective } \\
\text { translational lift. } \\
\text { 4. Touching down at excessive groundspeed. } \\
\text { 5. Failure to touch down at an appropriate attitude. } \\
\text { 6. Maintaining poor alignment with the direction of travel during } \\
\text { touchdown. }\end{array}$ \\
\hline $\begin{array}{l}\text { Slope Operations } \\
\text { (Figure 16) }\end{array}$ & $\begin{array}{l}\text { 1. Failure to maintain heading resulting in a turning or pivoting motion. } \\
\text { 2. Turning the rotor/tail into the slope. } \\
\text { 3. Lowering the downslope skid too rapidly (Roll). }\end{array}$ \\
\hline
\end{tabular}


A pilot generally is trained to perform high altitude and low altitude reconnaissance to identify any necessary threats while approaching into a potential landing zone and when close to the landing point [26]. The following high-level pilot duties are followed when landing;

1. High Reconnaissance, with the objective of determining the direction, the speed of the wind, a touchdown point, sustainability upon landing and axes of approach and departure [26].

a. Determining a landing point

b. Assessment of weather, wind direction, terrain conditions, and obstacle understanding.

c. Initiation of approach (into the wind is preferable), approach angle and speed.

d. Descent while maintaining visual on the possible landing zone while decelerating and maintaining descent angle.

e. Check for Go/No-Go for landing based on obstacle clearance.

f. Descend into the landing area.

2. Low reconnaissance, is performed while approaching the landing area, verifying what was observed in high reconnaissance. Observe and detect any new or smaller obstacles that may have missed in high reconnaissance (wires, towers, slopes, ditches etc.) [26, 28].

a. Once in hover phase or low altitude approach depending on which landing sequence is chosen, the pilot must look for smaller obstacles.

b. Obtain a clearer idea of any small obstacles or irregularities in a landing zone.

c. Decide on Go/No-Go before the effective translational lift limit.

d. If Go landing is confirmed, final descent must commence with the minimizing the amount of lateral, longitudinal and height drift.

When a helicopter is in the landing phase, numerous performance factors affect the creation of a brownout/whiteout. The intensity of brownouts and whiteouts depend on the disk loading of the helicopter, approach direction, and method of landing. In the presence of DVE, the dangers associated with common errors that occur can be greatly heightened.

\subsection{HELICOPTER FLIGHT DECKS, PAST, PRESENT AND THE FUTURE}

The 1940s was the introduction to helicopters operations into the aviation industry, mainly in the military context. The use and operations grew extensively over the next two decades [24]. Cockpit instrumentation 
for helicopters like fixed-wing aircraft started primitive with increased digitalization over time with advanced system integration. There has been a considerable divergence in the cockpits of fixed-wing vs. helicopters as their operational necessities were utterly different. As it is for aircraft, the basic required instruments for helicopter flight operation includes; attitude, altitude, airspeed, engine and rotor RPM, turn and slip indication, vertical speed, manifold pressure, magnetic heading information. To support navigation purposes of a helicopter, the radio control panel would contain Course Deviation Indicator (CDI). In older aircraft, all these instruments are presented as their independent entities in the traditional helicopter flight deck [27] via mechanical dials. Figure 17 displays an older Dial based UH-1B helicopter flight deck.

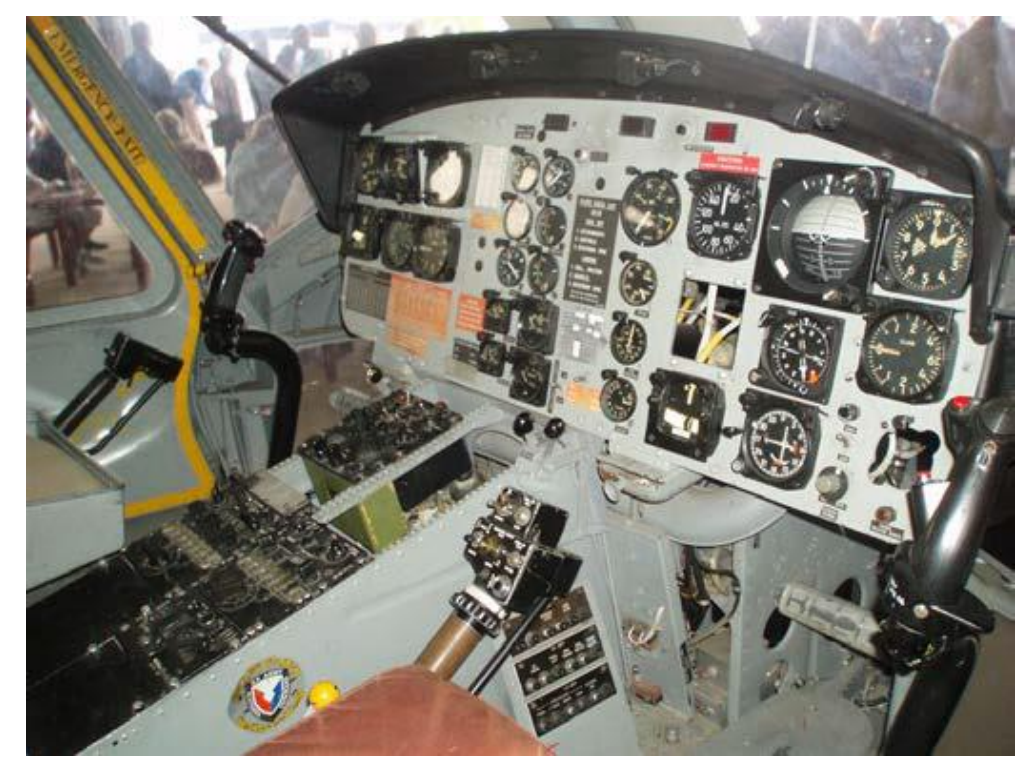

FIGURE 17: PICTURE OF AN OLD DIAL BASED UH-1B HELICOPTER FLIGHT DECK [27].

More modern glass cockpit systems (First introduced in the OH-58 Kiowa) would include the likes of Primary Flight Display (PFD), Multi-Function Display (MFD), Navigation Display (ND) and Cockpit Assistant System (CAS) Display. Digitalization in helicopter flight deck has generally been slower compared to fixed-wing aircraft, as the operational environment is entirely different. Helicopter undergo considerably more vibrations compared fixed-wing due to the main rotor and tail rotor, thus integrating sophisticated avionics and filtering the corresponding noise in the display systems can be challenging given the sheer number of systems and desired accuracies. Helicopters also require more complex avionics systems with higher levels of software certification and robust Line Replaceable Units (LRU) [27]. Figure 18 shows a more modern helicopter flight deck with digitalized displays. 


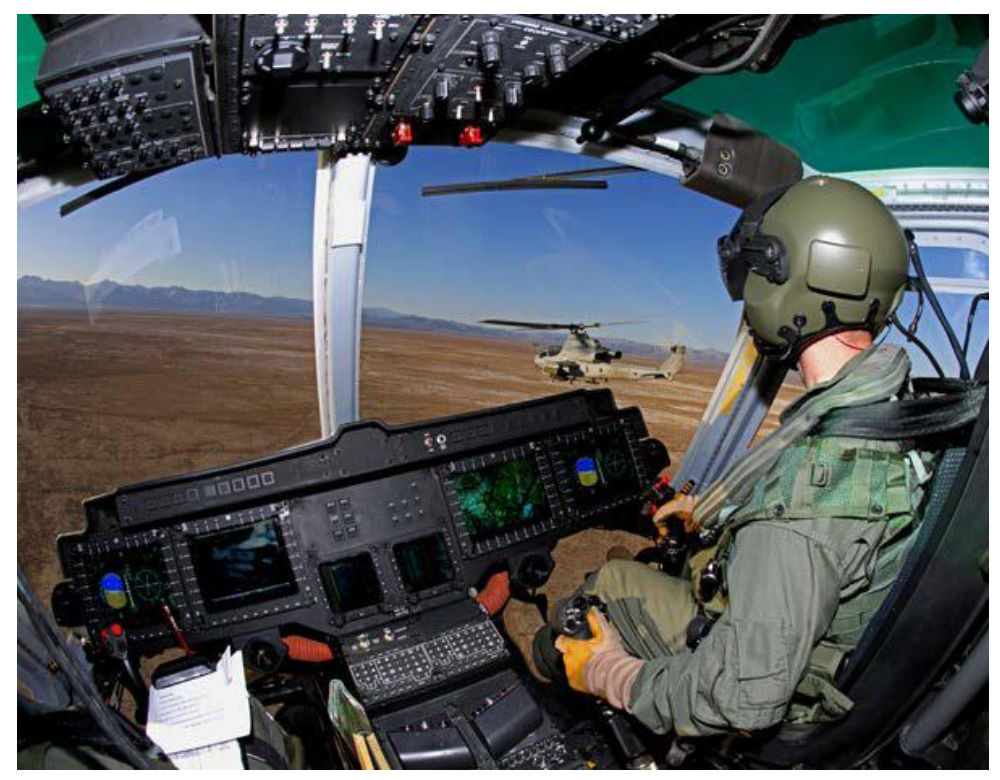

FiguRE 18 PiCTURE OF A MODERN UH-1Y DigITAL FLIGHT DECK [27].

In digitalized helicopters the flight deck is entirely different to pre-existing models, typically featuring two MFD for horizontal and vertical situational awareness a Multi Control and Display Unit (MCDU) for management of FMS for flight and mission planning. PFDs used in present day flight decks only consist of minimum requirement of airspeed, altimeter, turn, magnetic compass indicators [27]. The overall speed of the aircraft is shown with no breakdown in the lateral and longitudinal directions making it difficult to predict direction of drift. More display units are present for a more custom approach to show information that is required by the pilot and the mission, i.e., video surveillance, Radar/LiDAR information, target tracking, extended communication platforms. Helicopter human factor research revolves around the idea of increased safety of rotorcraft operations, all culminating as avionics systems which would be integrated on the helicopter. A comprehensive study by NLR highlighted 145 different technologies which can be mitigated helicopter accidents [27, 29]. Some of the vision-based systems are;

1. Digital range image algorithms for visual guidance in low-level flight

2. New terrain following guidance algorithms for rotorcraft displays (including SVS and EVS concepts)

3. LiDAR obstacle and terrain avoidance systems

4. Predictive ground collision avoidance using digital terrain reference navigation like the flight path.

5. Engine Backup systems 
6. Practical regime prediction approach for health usage monitoring system (HUMS) applications

7. Helicopter pilot assistant systems featuring flight planning and 4D trajectory optimization.

One of the critical components on any aircraft system is the presence of an Instrument Landing System (ILS). In aircraft landing sequences pilots use ILS to guide them to the runway with bearing and glideslope deviation representation between the aircraft and the runway. The ILS system, at any major airfield, consists of two critical components, the glideslope, and the localizer. Both the systems cast beams at frequencies between 108.1-111.95 MHz and 329.15-335.0 MHz respectively. The localizer transmits two lobes down the length of the runway to a certain distance that is varied based on the traffic around the airport [30]. Figure 19 depicts how the localizer deploys the signal lobes.

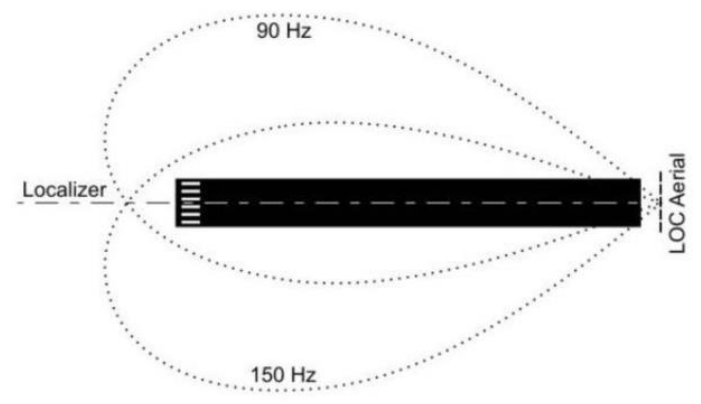

FIGURE 19:DEPICTION OF A LOCALIZER SETUP THAT WOULD BE PRESENT AT ACTIVE AIRPORT RUNWAY [31].

The Glideslope is generally not used far away from the runway; glideslope antenna is placed at a distance from the touchdown zone markers on the runway, offset by a small amount. Two lobes of signal like the localizer are transmitted at a 3-degree slope for aircraft. However, this depends on the installation requirements. Figure 20 depicts how the glideslope signals are deployed.

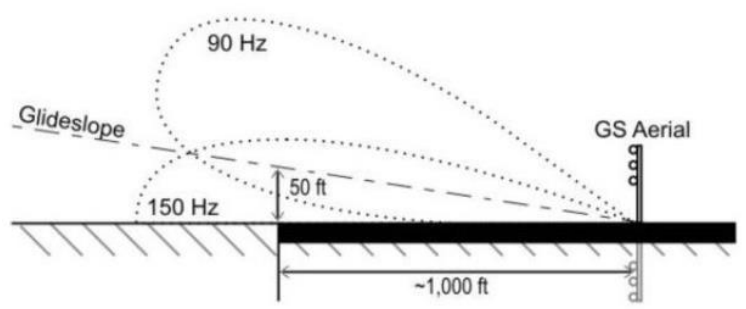

FIGURE 20: DEPICTION OF GLIDESLOPE SETUP THAT WOULD BE PRESENT AT AN ACTIVE AIRPORT RUNWAY[31].

The antenna, radio, and navigation equipment on the aircraft is responsible for the "catching" of the signal based on the prescribed frequencies and are selected by the pilot. An onboard antenna receives the localizer signal, from the Very High Frequency (VHF) and Omni-directional Range (VOR) radios to calculate the difference between the systems [27]. The incoming signal track is compared to that of the aircraft and is 
displayed on a 2D instrument (shown in Figure 21) in the cockpit which represents the heading and elevation deviation between the aircraft and the runway.

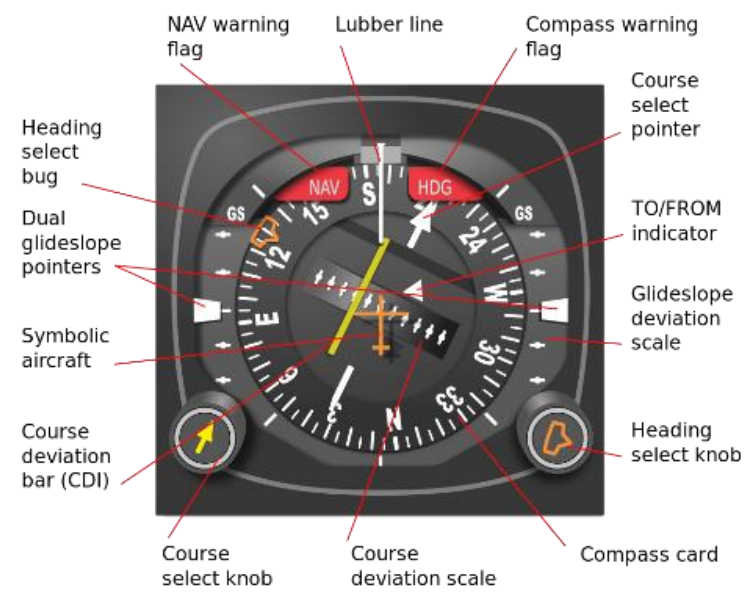

FIGURE 21: A HORIZONTAL SITUATION INDICATOR [27].

Similarly, in the case of DVENS, the development and final implementation of the symbology (2D and 3D, terrain texture representations and obstacle representation) are displayed on a glass MCDU for reference and providing higher situational awareness to the pilot.

\section{AUTOMATION IN AEROSPACE}

Aviation has grown to be highly automated, whether it be Autopilot systems or Full Authority Digital Engine Control (FADEC). Automation is an essential tool in reducing pilot workload and stress. A level of automation is also essential for pilots operating in DVE along with augmentation of missing VFR cueing. Automation can lead to substantial decrease in workload, however it can also be detrimental. Automation can harm pilots, both psychologically and psychosocially [32,33]. With excessive automation, pilots tend to become complacent and miss cues and duties. Without it, pilots are overloaded with tasks. Paired with a DVE operating scenario causes for very high workloads and stress levels to be induced. In odd occasions, pilots who are not fully trained for the automation embedded onto aircraft can make fatal mistakes, without information present about the system.

\subsection{SPECIFIC TO THE DVE SOLUTION}

\subsubsection{D SYMBOLOGY}

Helicopter flight instrumentation like any other flying vehicle shows a wide array of grouped information. Instrument grouping is significant to pilots and taught in ground school. It allows the pilot to compartmentalize the displays into three major areas of flight information. The three existing functional groups include control, performance, and navigation [34, 35, 36]. Table 2 provides a summary of different instruments and what groups they belong to. 
TABLE 2: TABLE SUMMARIZING INFORMATION CORRELATING TO INDICATOR [36].

\begin{tabular}{|c|c|c|c|}
\hline Indicator & Control & Performance & Navigation \\
\hline Power & $\mathrm{X}$ & & \\
\hline Attitude & $\mathrm{X}$ & & \\
\hline Angle of attack & & $\mathrm{X}$ & \\
\hline Airspeed & & $X$ & \\
\hline Altimeters & & $\mathrm{X}$ & \\
\hline Turn and Slip & & $\mathrm{X}$ & \\
\hline Vertical Velocity & & $\mathrm{X}$ & \\
\hline Horizontal Situation & & $\mathrm{X}$ & $\mathrm{X}$ \\
\hline Radio Magnetic & & $\mathrm{X}$ & $\mathrm{X}$ \\
\hline Bearing distance heading & & $\mathrm{X}$ & $\mathrm{X}$ \\
\hline Course & & & $\mathrm{X}$ \\
\hline Range & & & $\mathrm{X}$ \\
\hline
\end{tabular}

The military was the first user of HUD/HDD and 2D/3D symbology technology[30], to provide their fixed-wing aircraft pilots with immediate information they would need to make in quick decisions. The graphic elements and their proper function is based on research and experience. All the instruments can be compartmentalized into three different groups, which are listed below;

1. Control Instruments: These instruments display aircraft attitude and power; they directly reflect the response of the aircraft to the control column, rudder, and throttle inputs. These also reflect the "requested" values to attain specific performance or attitude conditions, like requested vertical speed, heading rate change etc [36].

- The pitch-and-roll rotational motions resulting from the stick movement are displayed via the attitude indicator.

- Either the heading indicator or the inclinometer displays the yaw rotational motion.

- Aircraft power is displayed via an engine instrument, usually either a tachometer or rpm gauge or a temperature gauge that reflects thrust capabilities. 
2. Performance Instruments: Performance instruments display the aircraft's response to control inputs (i.e., attitude and power. etc.), there is generally a latency after the commanded control input before they reflect the current state of the aircraft. The category of the performance instruments traditionally includes, the altimeter, vertical speed indicator, the turn and slip indicator, the heading indicator and the angle of attack indicator[36].

3. Navigation Instruments: Includes various types, of course, range and glideslope indicators, bearing pointers and waypoint depictions. These displays indicate the position of the aircraft relative to a selected navigation facility or a physical waypoint over the ground, with respect to a given coordinate system such as longitudinal or latitude. These are primarily associated with geographic orientation [36].

Early HUDs presented symbology like head-down instruments [37]. Generally, they displayed some form of the pitch reference with background scale. Eventually, other flight information was added to improve pilot performance and situational awareness (Information such as Angle of Attack (AOA) indicator, G indicator, Mach indicator, Roll) in time-critical situations [37].

The MIL-STD 1787C is a standard that ensures the primary information is displayed during all maneuvers and minimally on how they are displayed [37, 38]. Further considerations by the display designers must include features; color, size, movement, location, and arrangements, including the metrics with which to measure the display's effectiveness. There are some time-tested human factor principles of instrument design were established by Fitts.

1. Every display should be designed for quick perception.

2. Every display should be designed so that the meaning of its indication is immediately apparent.

3. A display should provide information that is precise and complete

4. Single and entire display systems should be as simple as possible

5. Different displays and controls should be easily distinguishable.

6. Displays that provide related information, or to which are referred to in rapid succession, should be grouped.

7. Controls and instruments should be designed so that they move in the "expected" direction.

8. Equipment should be designed so that continued operation for a number of hours results in minimum fatigue or loss of operator efficiency.

9. Principals' aspects of design, arrangement, and location of displays and control equipment should be standardized.

Military standard MIL-STD-1787B recommends color schemes for military display application [34, 37, 39]. Selective tones, and changes in color intensity or density have been employed on several attitude displays, and techniques were observed to improve the pilot's ability to quickly recognize the aircraft 
orientation during unusual attitude recoveries. As the pitch wither increases or decreases, the shade becomes lighter or darker, respectively, alerting the pilot to a pitch magnitude change. This intuitive presentation appears to reduce cognitive processing time compared to that required to read and interpret the numbers next to the pitch lines [34].

Table 3 presents a list of the colors used as per MIL-STD 1787, it recommends red be used for any warnings, system limits or threats going with the general human association with red representing stop or beware. The color amber or yellow be utilized for any warnings and situations which require the pilot's immediate attention. White or green is used for any case that is nominal and functioning accordingly, such as ground speed indicator, attitude indication. Blue is suggested to be utilized on the sky-on-attitude indicator, and brown or black as the earth-on-attitude indicator. Magenta is recommended for use with pilot-selected navigational information or subsystem values [34].

TABLE 3: RECOMMENDED COLOR-USE CHART FROM MIL-STD-1787 [34].

Function

Color Required

Warnings, warning- level flight envelope or system limits, Red

and dangerous military threats.

Cautions and other information with cautionary level of Yellow and Amber

impact, such as unknown aircraft tracks, TCAS threat

advisories, and scale marks indicating approach to

abnormal/out of tolerance conditions.

Sky-on-attitude indicator

Blue

Earth-on-attitude indicator

Brown or Black

Advisories

Blue or green

Selected menu options or captured modes, question marks White or Green

for missing data and status "normal"

Pilot-selected navigational information or subsystem Magenta

values (i.e. joker fuel, bingo fuel or altimeter setting)

Heads up Display (HUD) and Head down display (HDD) 2D symbology is a means to display important flight performance information to the pilot while maintaining eye outside the cockpit. They are generally transparent displays that present data not requiring the user to look away from usual viewpoints. HUDs 
provide the pilot with a mixed view of onboard flight information on a transparent display with real-world cues. Some of the significant advantages of using HUDs are;

1. Reduced Pilot workload, the overall piloting tasks require heads-up, outside-the-cockpit flight references.

2. Increased flight precision, the expanded scale of the HUD data and its overlay on the external visual scene allow the pilot to fly more precisely.

3. Direct visualization of trajectory, a conformal display allows the pilot to assess the a/c performance directly.

4. Increased flight safety essential flight information presented on the HUD reduces eyes-in -the cockpit during critical flight maneuvers.

\section{CLUTTER}

One of the issues with HUD display design is the inclusion of all the information that the designer thinks a pilot would possibly need; however, this leads to a cluttered display. Traditionally, display engineers sought expert and user opinion for guidance during the development of new flight displays, pilots (users) tend to have firmly held opinions and may have limited knowledge is display evaluation often limiting the design of the novel system to those concepts with which they are familiar with [40, 41]. The following set of questions highlight the design process and underline the justification of a new display or components to a new display;

1. Are pilot information needs satisfied by the display?

2. What data does the pilot need that has not been provided?

3. Can the average pilot obtain what is required easily?

4. Does the display conform?
a. To the real world?
b. To other cockpit displays?
c. With the pilot habits and skills?
d. With the required decisions and actions?

Every information element shown on a HUD must serve a purpose and lead to improved performance. While all displays need to minimize clutter, it is more critical for see-through displays. HUD symbols are presented in the pilot's view of the real world; obtrusive symbology should be kept to an absolute minimum. The general arrangement (location within the field of view) and the algorithms driving the symbols are more important than the details of the symbols themselves.

\section{EXISTING 2D SYMBOLOGY FOR DVE}


To overcome operations in DVE, numerous 2D brownout and whiteout symbology sets have been developed. The most well-known symbology sets were developed for $2 \mathrm{D}$ views forward viewing. The symbology sets were developed to assist pilots in landing during DVE; they present the pilot with basic flight parameters, speed, attitude, and acceleration cues.

Some of the existing 2D symbology sets are the non-conformal Brown Out Symbology System (BOSS), developed by the U.S. Army Aviation and Missile Research Development and Engineering Center (ARMDEC) (shown in Figure 22) and modifications [8, 41]. DEVILA (shown in Figure 23) was developed by European Aeronautic Defence and Space (EADS) of Germany and JEDEYE (shown in Figure 24) was developed by Elbit Systems in conjunction with German Aerospace Center (DLR) [8, 41]. These symbology sets were developed to provide the pilot with control, performance, and navigation parameters of the helicopter. Research performed at DLR presented suggested the 2D concepts alone does not provide sufficient guidance quality as they may lead to attention tunneling, cluttering and confliction of optical flow in the near and far-field domains.

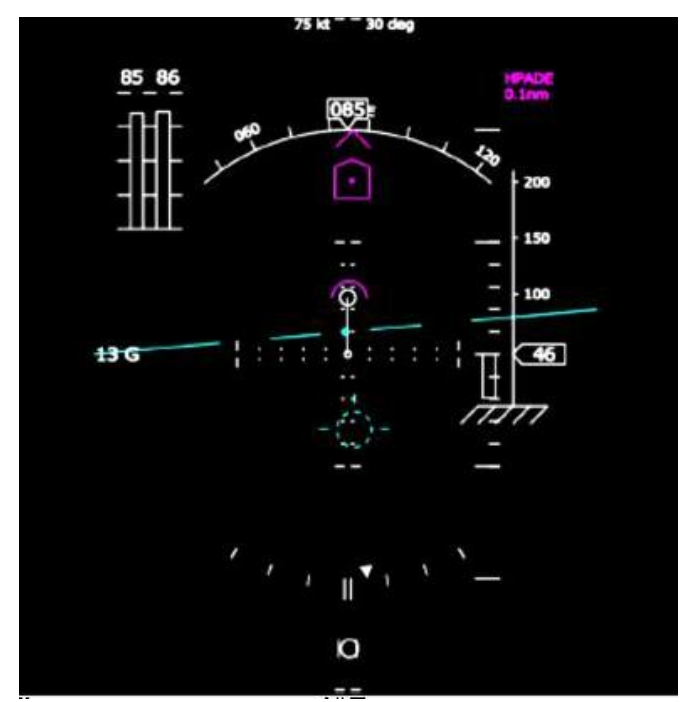

FiguRE 22: BRownOUt SyMBology SYSTEM (BOSS) [8, 41]. 


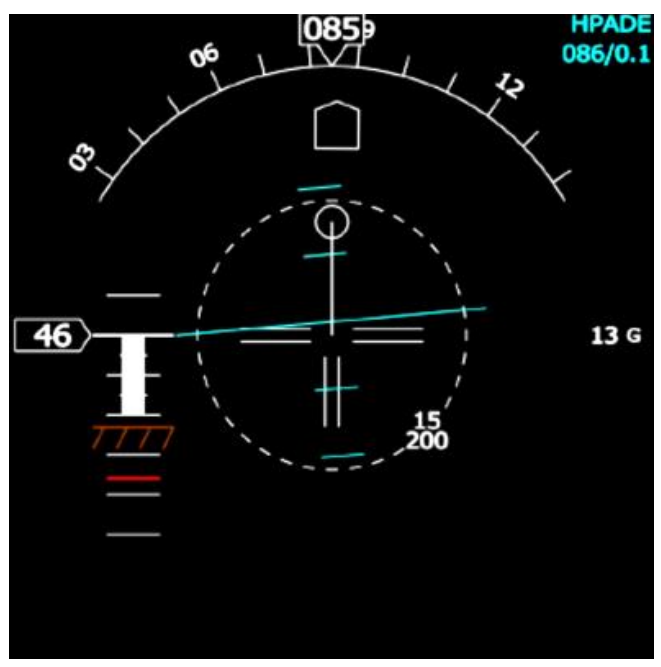

FIGURE 23: DEVILA SYMBOLOGY SYSTEM, DEVELOPED BY EADS, GERMANY [8, 41].

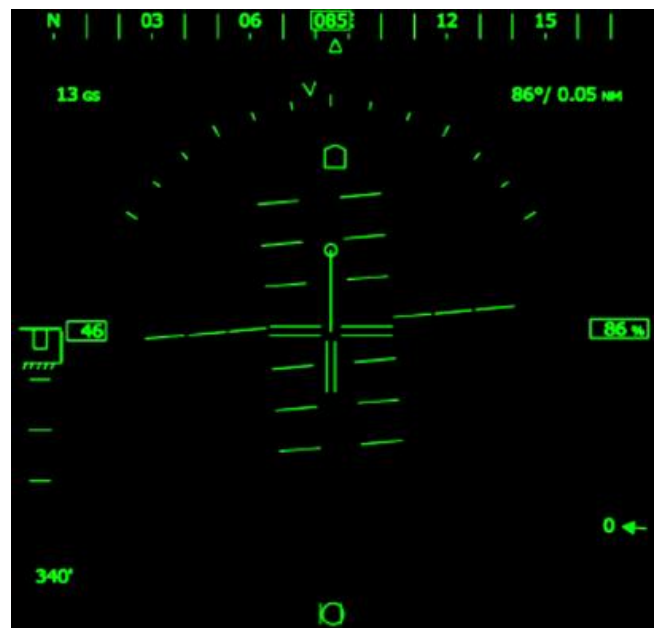

FIGURE 24: JEDEYE CONCEPT WAS DEVELOPED BY ELBIT SYSTEM TOGETHER WITH DLR, GERMANY [8, 41$].$

The three different DVE orthographic symbology highlighted above are developed by different organization but are based on solving the same issues of pilots SA. The basis of the symbology is very similar, they aim to provide the pilot with the helicopter's control, performance and navigation information. The major differences are the extent of graphical elements shown, the BOSS is a more cluttered graphical footprint, over the DEVILA and JEDEYE symbology. The BOSS consists of a roll indicator at the bottom quadrant of the symbology, whilst DEVILLA and JEDEYE embedded the roll indicator into their heading indicator. The roll indicator in the lower quadrant can be disturbing on indistinguishable when overlaid with sensor data and images. However, the cohesion of roll and heading indicator in DEVILLA and JEDEYE can be hard to differentiate, the respective roll and heading tape. The JEDEYE concept consists of a pitch indicator along with the lateral/longitudinal draft cueing, while the BOSS symbology is composed of two stages of hover and cruise symbology distinguishing the information required in the two phases of flight. 


\subsubsection{D SYMBOLOGY}

Traditionally 2D orthographic symbology is displayed in the pilot's field of view while looking out of the cockpit. However, with technology advancing the integration of 3D conformal images and 2D orthographic HDD symbology are being a common norm to create non-transparent fused displays.

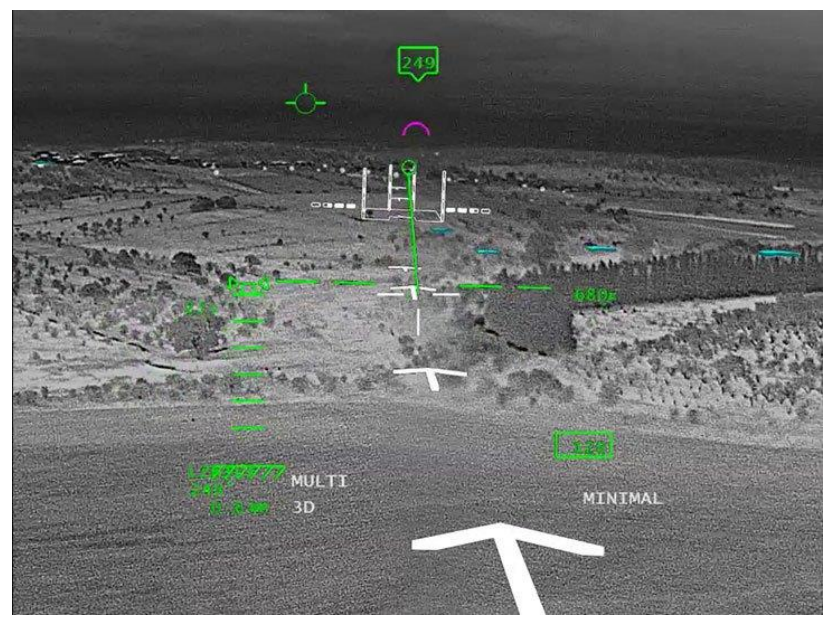

FIGURE 25: A 3D SYMBOLOGY DISPLAY OVERLAID WITH HELICOPTER INFORMATION [42].

Figure 25 illustrates an example of 2D orthographic symbology overlaid over a 3D conformal thermal image. Integration of such sources of information provides an exceptional level of situational- awareness to the pilots in DVE. A 3D thermal image can be used to identify obstacles in a fixed field of view, overlaying it with a 2D symbology would present the pilot with the required helicopter control, performance, and navigation. 3D symbology is a crucial component as it provides a pilot of their natural flying tendency, out of the cockpit view or VMC, simulating a heads up to eyes out flight [35]. During a typical landing sequence, the pilot perceives necessary information required for landing from outside world cues to understand the helicopter's landing zone, approach speed, and drift, attitude, and altitude. Pilots use simple cues like micro-textures of grass, rocks, etc. to judge and perceive their motion/drift respective to the terrain. In DVE such understanding is not possible due to loss of all visual cues, 3D cueing symbology would be placed in a conformal manner in the real world. 3D symbology is generally used in conjunction with 2D symbology to provide an idea of both the operating environment and information of the helicopter's control, performance and navigation. There have been numerous previously developed 3D cueing symbologies, as seen in Figures 26, 27, 28. There are three popular 3D Safe Landing Zone (SLZ) symbology, 3D Brown Out Symbology System (BOSS) developed by the U.S. Army Aviation and Missile Research Development and Engineering Center (ARMDEC). Brownout Landing Aid System Technology (BLAST) developed at the U.S. Army Aeroflightdynamics Directorate (AFDD) along with BAE systems [18]. The Sferion and Airbus Defense and Space developed 3D conformal HMD Symbology. The three highlighted 3D symbology sets serve to provide various cues to the pilot. 


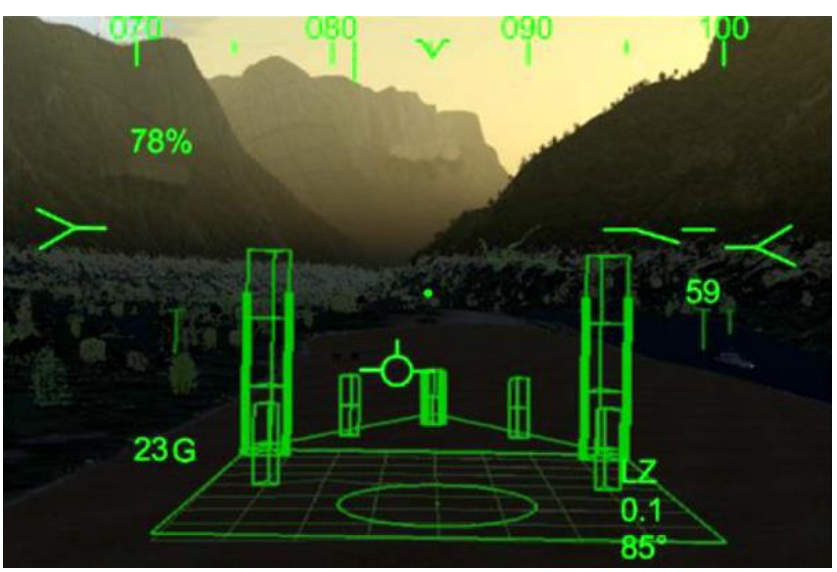

Figure 26: THE 3D AND 2D Airbus Symbology [8, 43].

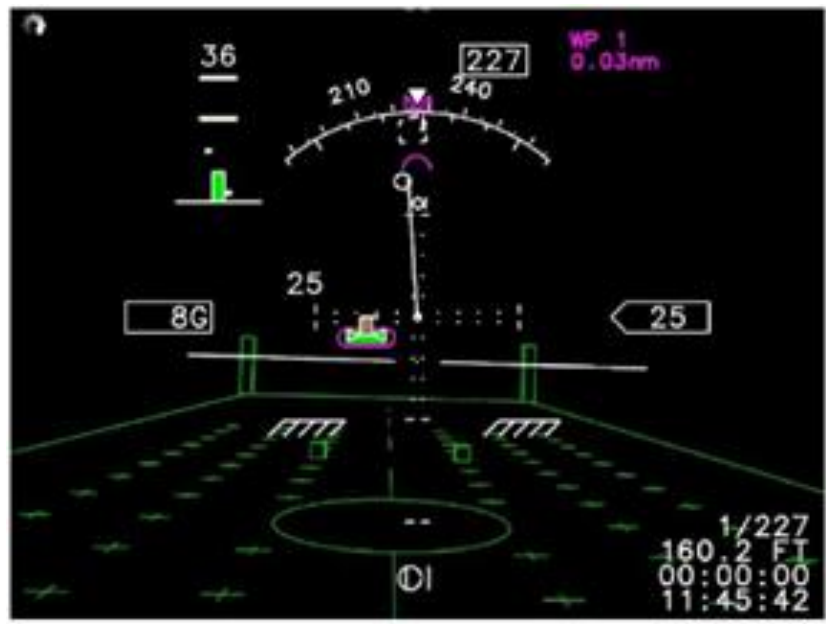

FIGURE 27: 3D AND 2D BOSS SYMBOLOGY DISPLAY [8] [43].

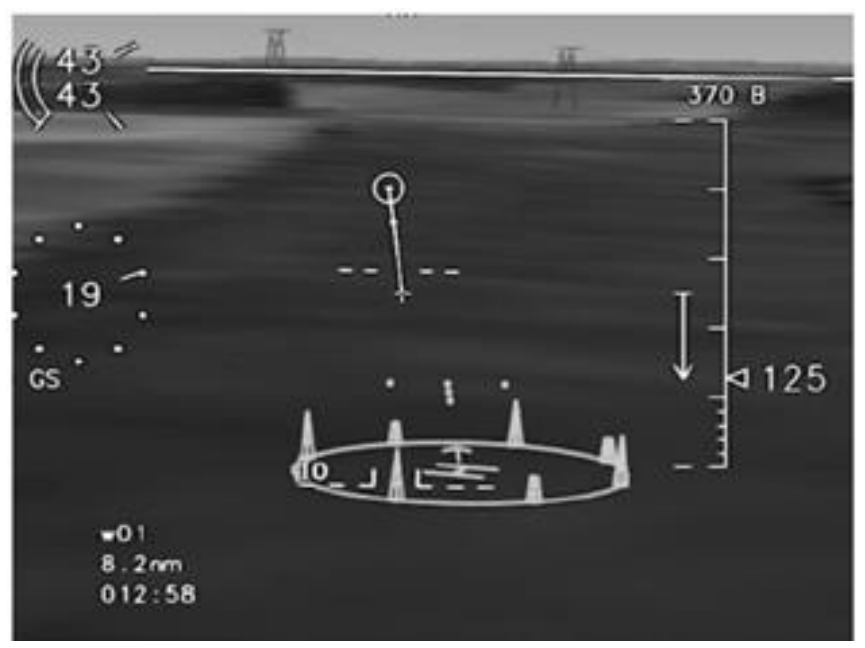

FIGURE 28: 3D AND 2D BLAST SYMBOLOGY DISPLAY [8, 43]. 
Similar to the 2D symbology highlighted, the three-3D symbology presented show distinct differences. The Airbus symbology in Figure 26 is and 3D BOSS symbology in Figure 27 are very similar in components. The Airbus 3D consists of more vertical pillars which help the pilot understand the lateral and longitudinal deviations. Another distinguishable difference between the Airbus and other 3D symbology are, the pillars at the corners are dynamic and populate with color to represent the radar altitude of the helicopter. The BOSS symbology consists of only two pillars which the pilot would use to perceive lateral and longitudinal deviation. The 3D BLAST symbology is completely different to the BOSS and Airbus symbology. BLAST is represented via radial symbology, with respective pillars for various drift cues. The BLAST symbology consists of an ' $\mathrm{H}$ ' to signifying the center of the LZ. An arrow is used to represent the wind direction, and all three symbology sets contain a circle in the middle of the LZ to represent the slope of the terrain.

\subsubsection{SECONDARY VIEW MODES AND TERRAIN TEXTURES}

The basis of Situational Awareness (SA) is built around the idea of a pilot having enough information to successfully perform a task or objective in the event of something unforeseen occurring. Thus, when a pilot is flying into a new area, updating SA information is highly beneficial. Extra view modes can help the pilot attain more SA about surrounding terrain, dynamic/static obstacles, passed landing zones, and cultural information that help make sound mission-centric and operation centric decisions. Addition of a new view mode emulates a map/chart of operation area that would be used on any mission and presenting real-time updating information about the environment around the pilot of safe/unsafe landing zones.

A study conducted by the Airforce Research Laboratory, "Landing an H-60 helicopter in brownout conditions using 3D-LZ displays", 2010 at the Yuma Proving Grounds in the United States explored the effects of using multiple view-modes or angles to study the improvement in landing operations of helicopter pilots [44]. The study saw four pilots accomplishing 23 successful landings out of 31 . The test cases consisted of three different types of displays; single, dual, and switched displays. The single display presented a forward view, the dual-display presents a top-down or planform view around the helicopter, and the switched display toggles between forward and planform views. The switched platform transition would occur based on pre-determined conditions of helicopter altitude or speed or pilot choice. Each display view was subjected to its orthographic $2 \mathrm{D}$ symbology, that would show the pilot required information at the event state. The following results were gathered in the testing;

1. In terms of measured landing accuracy, the dual display format performed best, followed by a switched display. With 6 and 3 landings within a 50ft radius desired landing zone respectively [44].

2. In terms of measured vertical speeds in low altitude, the dual display and switched display performed well. The dual display was well within the desired vertical speed range, as opposed to single and switched displays which were within the desired zone and adequate zones[44]. 
3. In terms of measured lateral speed, most desirable and adequate landings were made with the switched display, followed by the dual display [44].

4. In terms of measured forward speeds, Dual display provided the most desired forward speed variations followed by a switched display [44].

5. Two out of the 4 pilots preferred to fly with the single display and 1 with dual and 1 with switched displays [44].

6. The handling quality ratings showed a dual display to provide better performance. Dual display mode has the lowest pilot performance demand (mental, temporal, physical, performance, and frustration) [44].

Thus, this study provides a means of showing which type of display system is most beneficial for DVE operation, and it was concluded that the dual display type is best suited in provided overall SA [44].

\section{TERRAIN REPERSENTATION VIA TEXTURES}

Pre-existing terrain Common Databases (CDB) are used in the simulation to portray terrain developed with Digital Elevation Maps (DEM). DEM provide and visualize vector data which represents the relief of a surface between points of known elevation. DEM CDB provide the perception of geographical entities and not the necessary perception of terrain that pilots would retrieve flying in the real world. Pilots can deduce altitude, attitude, velocity, slope, and cultural information from the terrain while flying in real-time. Simulation DEM/CDBs fail to provide an accurate representation of changes in terrain, DEM data is expensive with mounting costs for higher resolution information. Computer-generated "synthetic" terrain from CDBs can be improved through texturing [45]. Synthetic Vision Systems (SVS) or Enhanced Vision System (EVS) are utilized to provide pilots with the necessary perception of terrain information and flight instrumentation (navigation, control and performance) [46]. Providing the necessary detail to a structure that allows for the proper perception of altitude, attitude, slope, velocity, and cultural information relatively are essential for improved performance. An alternative method to texturing is to detail via shadowing with precise and high resolution data [45]. However, this lacks detail for smaller elevation changes which would be embedded within the shadow of a more significant elevations and would require higher calculations of system. The method of shadowing also takes a larger amount of computing power drawn from performance dedicated to other components of the development. LiDAR, though, can identify zones as being safe/unsafe, cannot in real-time update scanned terrain without the expense of substantial graphic and computational power [7]. The initial representation of the terrain is fixed and thus needs to provide an optimal perception of surface attitude.

Pilots must perceive terrain slope, altitude, and attitude while flying, landing, or takeoff of a helicopter. Under/overestimation of the slope, altitude, and attitude can cause rollovers in critical flight scenarios such as hover and descent. Thus, the main terrain characteristics outline the design goals for texture pattern use, 
textures that allow for the precise perception of slope, attitude, and altitude will provide the pilot with the necessary understanding of terrain characteristics. Gibson was the first researcher to propose the idea of shape perception from textures in 1950 [47]. Gibson suggested that the human visual system uses the texture density to estimate orientation relative to the viewer's viewpoint on corresponding surfaces. The effectiveness of texture in helping perceive the terrain can allude to factors such as the inline patterns, texel geometry (Shape of individual pattern element), symmetry, scale type and regularity of texture [48].

The perception of a slope can be attributed to three major types of distortions that show changing the slant of a drawn surface normal. Scaling of the perspective of texels in texture, can increase/decrease the size to show change from a reference texel, can be useful in showing slope change [44]. Foreshortening is a change in the ratio of vertical and horizontal dimensions of a texel, can be used similarly to scaling to show perspective [48]. The density of texels is the number of texels within a specified area and can show a highly dense texelated zone to be a high slope [45, 49]. Figure 29 visualizes the three distortions which affect perception. Figure 30 shows different examples of textures properties.
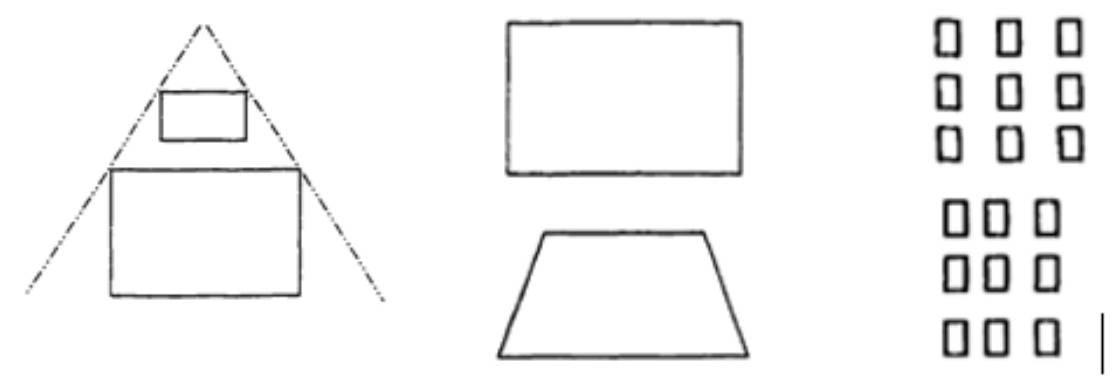

FIGURE 29: VISUAL REPRESENTATION OF THE THREE IMPORTANT FACTORS, DENSITY, FORESHORTENING AND SCALING, $[45,49]$.

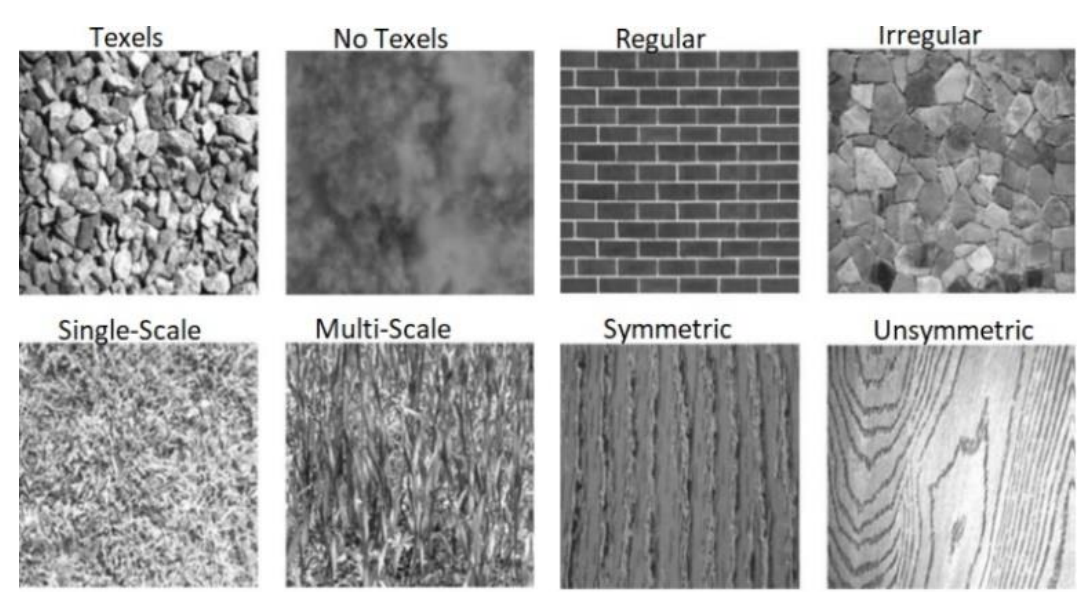

FigURE 30: VARIOUS PROPERTIES OF TEXTURES [47]. 
Rosenholtz found that testing results showed that the subject makes use of both deviations from isotropy and texture gradient/distortion as cues to recover shape. Thus, subjects used distortion of texture, and deviation from isotropy both equally [48]. A study conducted by Sunghee Kim et al, explored the effect of orientation in textures has on perception, they tested singly oriented (D-1 in Figure 31), doubly oriented (D-2 in Figure 31) and single orientation line with integral line convolution (Lic-like) ( (D-3 in Figure 31)) type textures. It was found the doubly oriented two-directional and lic-like textures provided significantly higher performance in judging attitude over singly oriented textures while no-texture shading seemed to significantly decreased judgment [50]. Figure 32 shows the 3 different textures overlaid onto a 3D saddle model to visualize the effects of perception from textures in Figure 31 with corresponding labelling.

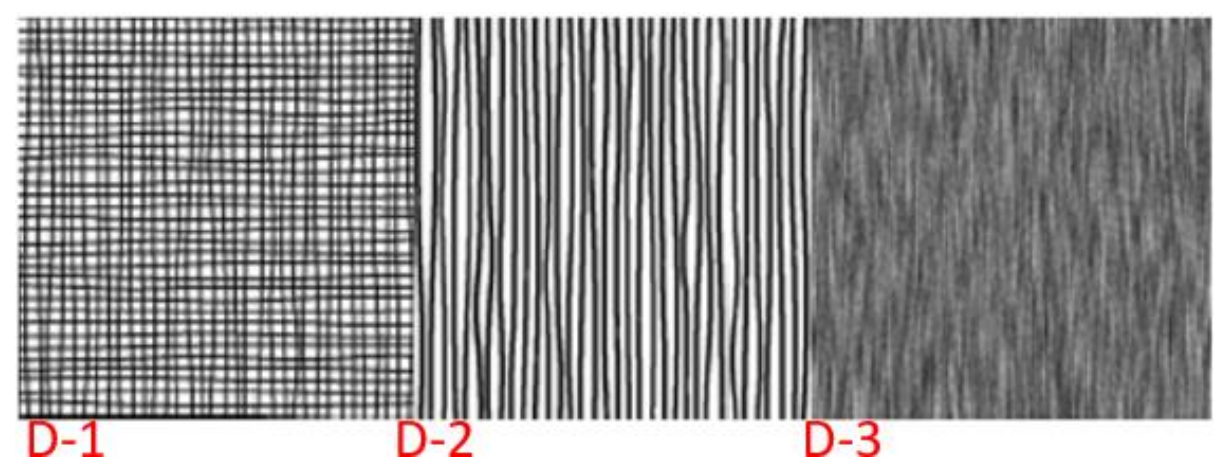

FigURE 31: DUAL DIRECTIONAL (D-1), UNI-DIRECTIONAL (D-2), AND MULTI-DIRECTIONAL(D-3) TEXTURES [50].
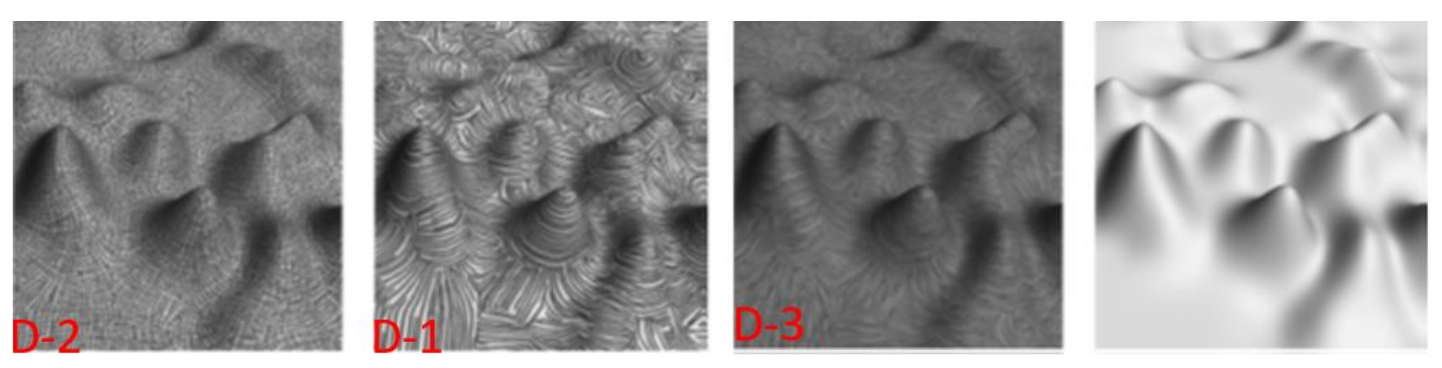

FIGURE 32:OVERLAID UNI-DIRECTIONAL, DUAL DIRECTIONAL AND MULTI-DIRECTIONAL TEXTURES PATTERN ONTO A 3D MODEL TO OBSERVE PERCEPTION OF DETAIL FOR A 3D MODEL WITH DIFFERENT TEXTURES FROM FIGURE 31[50].

It was found that performance was better in cases where textures carry information about distance along principal direction than in cases where patterns only carry information about the direction [50].

A study conducted by Kim. S et al. looked at designing an ideal texture pattern to an arbitrarily curving surface to perceive its shape accurately. Several stimuli were used, 1-directional, 1-directional clockwise 45, 1 directional 90 deg., 2 directional, 2 directional 45 deg., 3 directional (first and second principal directions), 2 directions plus a diagonal direction), in-swirling, and filtered white noise [51]. 


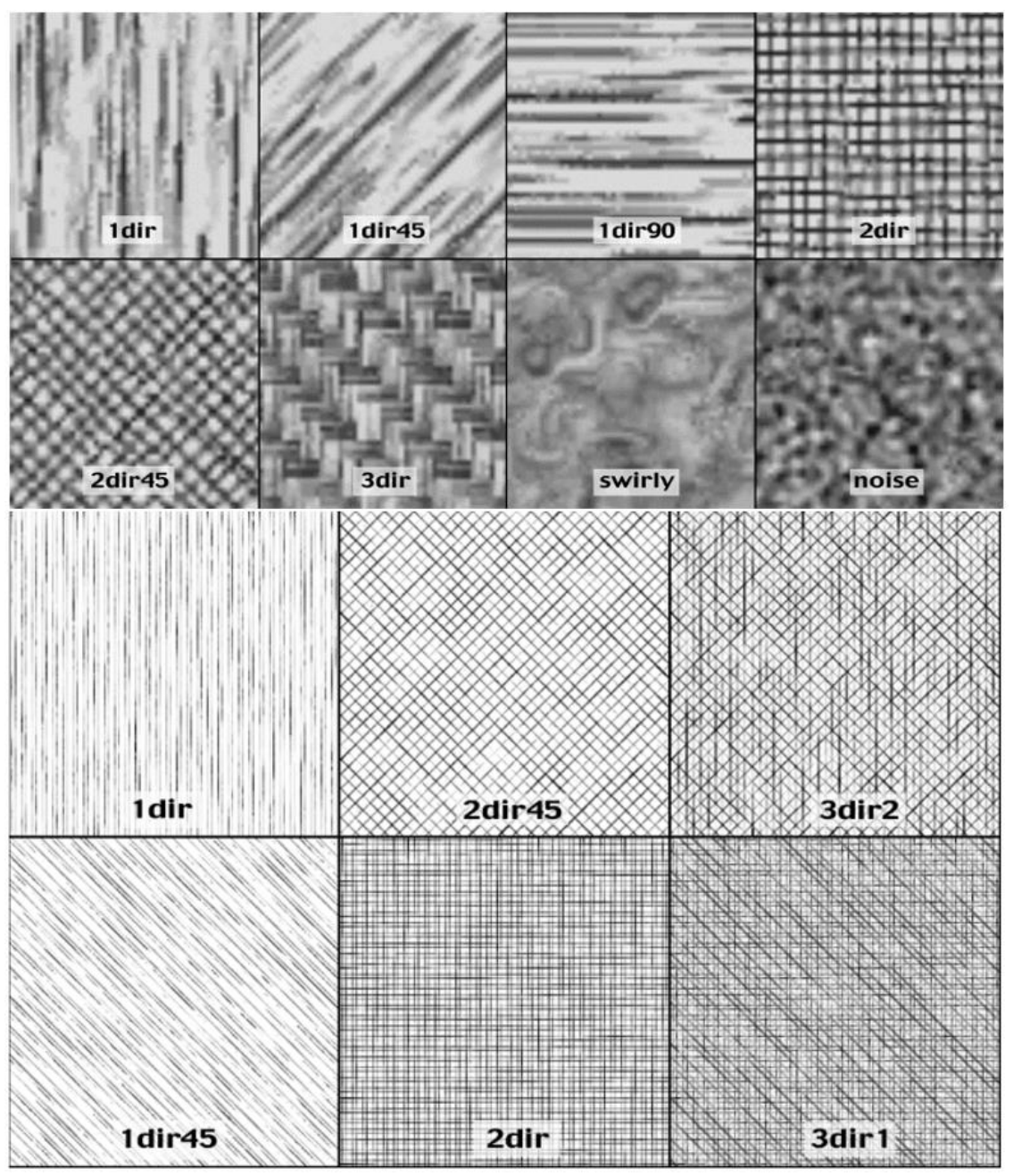

FigURE 33: TEXTURE PATTERNS WHICH WERE TESTED By KIM. S ET AL [51].

This testing sequence asked participants to perceive a shape to its actual shape; participants were asked to report on a perceived shape of a 3D structure. Deviation in the reported results was used to classify the effectiveness of texture in representing the actual shape of the 3D figure. Saddles, spheres, and ellipsoids were considered shapes as they provide a variety and variation in curves, slopes, and altitudes. The following results were observed in the performance of the testing.

1. Shape perception was better facilitated in bi-directional "principal direction grid" pattern than other patterns [50].

2. The perspective projection was required for accurate surface orientation classification.

3. Shape classification accuracy was higher when viewed at an oblique (perspective) than "head-on" (orthographic) [50].

4. Shape classification accuracy was equally good under for test patterns where components followed either first or first and second principal direction grids [50]. 
5. Extra information along more than the principal directions does not seem $100 \%$ safe.

6. Better surface orientation judgments are associated with strong directional texture patterns (1dir, 2dir, 2dir45, 3dir, and 1 dir90 in that order, which are shown in Figure 33) [50].

The second set of experiments showed that not all principal direction textures were equally useful for showing shape, and that subtle differences in texture characteristics could have a significant impact on the shape perception judgments.

A study by Thomas. B and Andrew H. S identifies a realistic scenario, Contrary to previous studies of using contour lines and grids in static environments; this study explores human factor experiments designed to study the relative effectiveness in dynamically changing surfaces. Evidence indicates that there are differences in shape perception between static and dynamic environments. The overall goal is to convey relatively 3D shape, absolute and relative sizes and rates of change. To represent busy scenes in which small changes are not overlooked, the stability of the derived textures as the surface deforms, pre-existing features can turn to be erratic in the presence of motion of change for the environment around it. In a regular grid texture, the cues can come from angles between the lines of two principal directions. By use of contour lines, motion cues appear from the expanding and contracting lines about a deformation. Contour lines are useful in obtaining attention to subtle changes[52]. The following discoveries were made in the performance of experiments;

1. Shading, though a strong cue it can be prone to error and applying texture has been found to be more effective[52].

2. Grids excel at providing the three cues; density, foreshortening and scaling due to consistency and regularity in texels. Grids were found to be powerful enough to overcome motion parallax cues when they conflict with one another. Contour lines provided similar cues however had serious ambiguity as to whether is it a result of a change in distance/orientation of a surface, or merely changing slope[52].

3. Sweet and Ware found grids to be more effective than contour lines for perceiving surface shape and curvature; except in top-down plan view mode, contours were found to be far more effective[52].

4. Lines are drawn horizontally (relative to the view direction) across the surface provide excellent curvature information but can only be used in the case where the scene is viewed from a static location[52].

5. In a second experiment, the relative attention capturing abilities between grids and contour lines was studied, it was found that contours performed best for changes with larger widths. For small/medium width changes, their advantage over plain shading and grids diminished[52]. 
A third experiment conducted determined that modifying a traditional rainbow color scale to be banded into discrete color bands instead of smoothly interpolated to produce color-based contours. It was found that the banded rainbow texture performed significantly better than the smooth rainbow texture. For changes with small widths, smooth rainbow texture performed significantly better than the banded rainbow texture for large and small depression[52]. In summary, from 4D visualization, contour lines performed strongly over grids and plain shading, indicating they were well suited for conveying sizes of changes in the dynamic surface. This study contradicts their findings of grids being superior to contours via the application in dynamic surfaces. It showed that structured cues could perform differently in static vs. dynamic situations[52]. The study involving the findings in a top-down or planform view is highly beneficial for an extended view mode.

A study by Velisavljevic and Elder looked at isolating roles of texels, scale structures, global symmetries and regularity with respect to the surface texture. The study concentrated in the perception of static surface's tilt/slant and found orthogonal linear structures lead to the most accurate judgment of surface tilt/slant [47]. This further supports the excellent performance of grids as applied to static surfaces. Researchers found that orthogonal linear structures are an essential for accurate surface attitude judgment giving more support to using grids for surface shape perception [47]. The textures testing by Velisavljevic and Elder are shown in Figure 34.
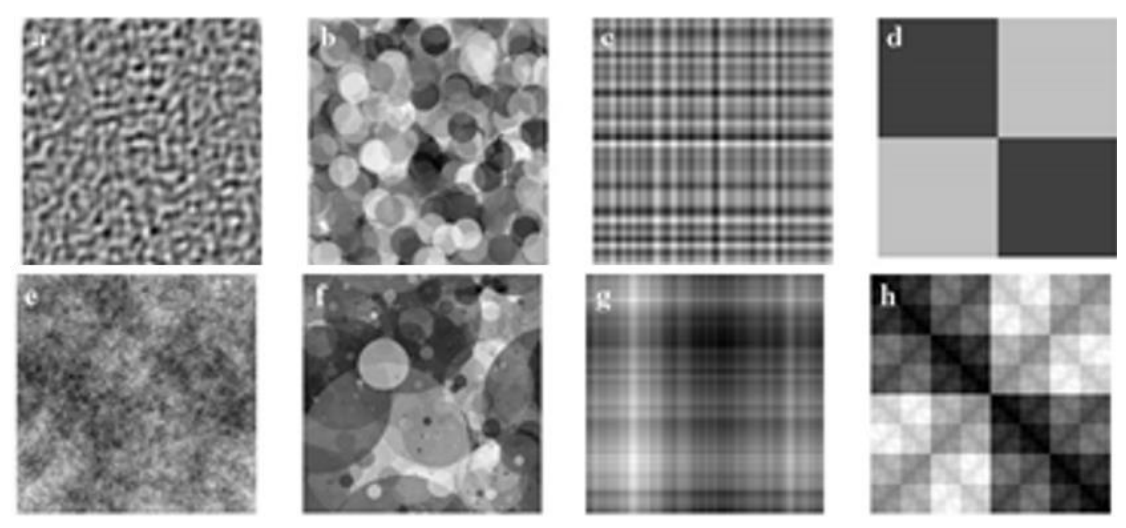

Figure 34: THE TEXTURES TESTED By VeLISAVLJEVIC[47].

Studies show that attitude perception for textures composed of well-defined texture elements may be more accurate than for textures with less well-defined texels. Surface textures comprised of single-scale texels would only provide useful information over a limited range of viewing distances. Multiple scale textures may support surface judgments over a broader range. Newman, Whinham, and Mac-Raw found that regular textures provided more accurate impressions of slant similar to Kraft and Winnicl and Gibson significantly over irregular textures [47]. The experimental results showed that;

1. Judgment of surface attitude based on featureless textures was found to be very poor [47]. 
2. Performance improves dramatically with the introduction of texels, random frequency structure or orthogonal linear structure [47].

3. For symmetric texture containing orthogonal structure, the operational range of viewing distances is greatly extended when its structure is present in multiple scales [47].

4. Optimal performance is obtained with symmetric textures containing orthogonal linear structure, whether or not the textures are spatially regular or contain well-defined texels: Orthogonal linear structure appears to be a necessary and sufficient property for accurate surface attitude judgment [47].

The studied resources summarize the following factors improve surface perception, and they lead to be the design features for the formation of a new texture [47].

1. Doubly oriented two directional provided greater performance in judging attitude.

2. Textures carry information about distance along the principal direction.

3. Symmetry seems to be an important character, with radial symmetry providing greater understanding.

4. The perspective projection was required for accurate surface orientation classification

5. Grids were found to be powerful enough to overcome motion parallax cues

6. in top-down plan view mode, contours were found to be far more effective.

7. Optimal performance is obtained with symmetric textures containing orthogonal linear structure Development of a new texture would be based on the outlined principals found over different studies. 


\section{CHAPTER 3.0 HUMAN FACTORS AND HUMAN MACHINE INTERFACING}

Human Factors is a study that accounts for a multi-disciplinary understanding of the interaction between

operators and instruments or operators and equipment. Human Factors is researched to improve the safety of systems, flexibility, and efficiency in operational environments $[53,54,55,56]$. Consideration of Human Factors increased in aviation with the rapid growth in aircraft in World War II. The importance of the human aspect of flying became evident in aviation with the advent of more situational representation instruments. The development in aviation also started to concentrate on the instrumentation and human-oriented design principles like Human-Centered Design [54].

\subsection{HUMAN CENTERED DESIGN}

Human-Centered Design concentrates on the development of interactive systems via application of human factors, ergonomics, cognitive studies, and usability techniques. According to ISO:210:2010 standard [55] to accomplish a Human-Centered Design and development, four major entities are important. A usable system allows increased productivity, reduced errors, reduced training and support, improved acceptance and enhanced reputation [55]. The first element of human-centered design is understanding the context of use and specification of the user domain, including the consultation of domain specialists and primary users. The second component is the involvement of users in the design process, a detailed plan to specify userrequirements $[54,55]$. The third aspect is the application of specialized human factors techniques to produce iterative design solutions followed by the fourth, which is the proper evaluation of design to validate the process $[54,55]$.

An application method popularly used for analysis is the Hierarchical Task Analysis (HTA), HTA is used as an initial step for a majority human factor application for the detailed objective breakdown. HTA calls for a detailed breakdown of objectives into many sub-objectives to build a plan for design or performance $[57,58]$. In the context of design, it is a formidable tool, able to provide detailed outlines of a design process in hierarchical order. A significant objective can be broken into simpler steps and algorithms for accomplishment. Clear overall goals at the system architecture level can be well defined. Individual goals can be further described by using flow charts highlighting an algorithm which would need to be implemented as seen in Figure 35. In the case where a clear definition does not exist, and a problem statement is available the HTA is not necessarily the best available option. 


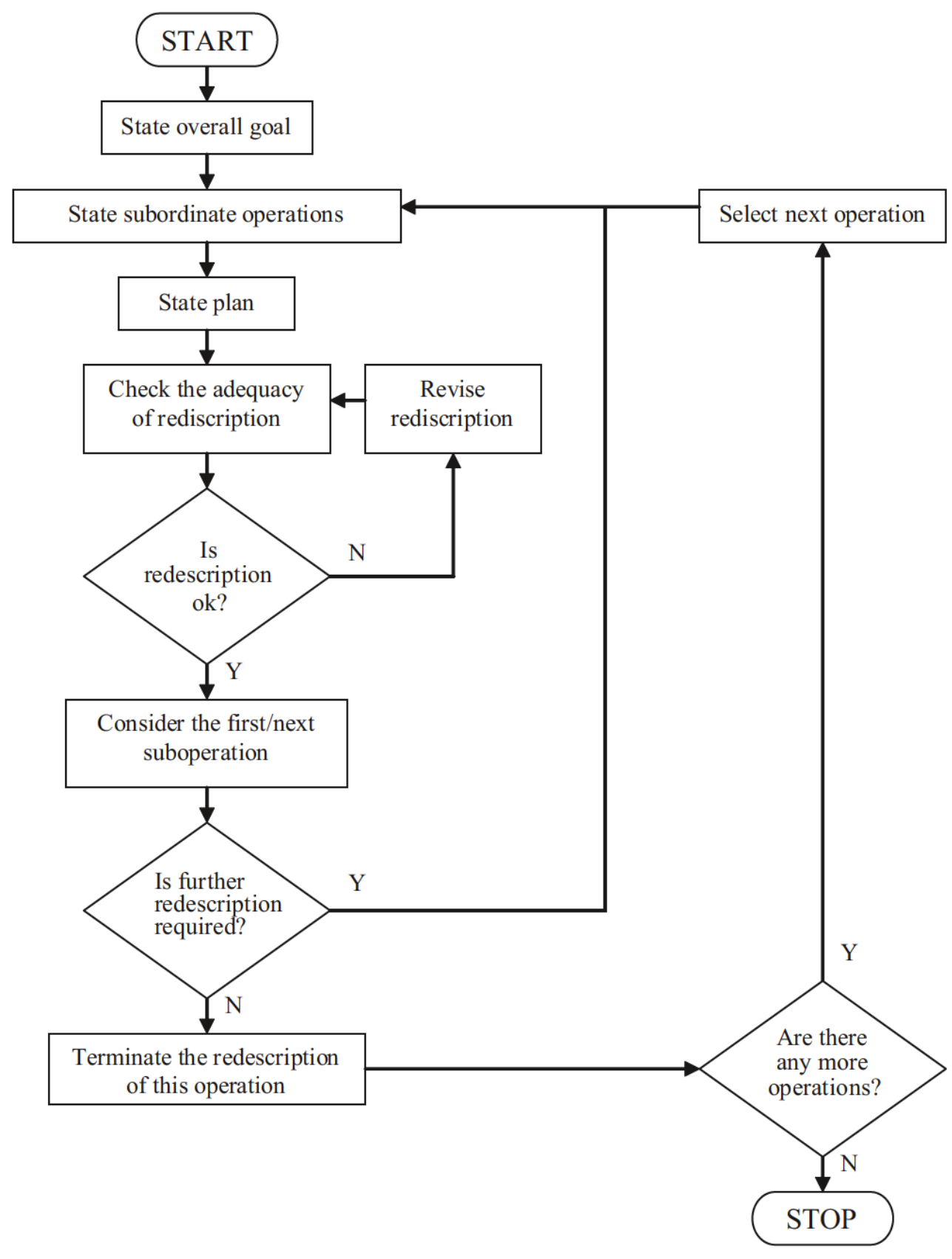

Figure 35: The Hierarchical TASK ANalysis Process Flowchart [54].

A general problem statement can revolve around an array of conditions that need to be represented. The developmental goals can remain unclear; thus, a reversed workflow can be utilized to identify apparent problems from which proper objectives can be formed. The Taxonomic Aircrew index is utilized to find where a pilot could have made a mistake that led to catastrophe, using the taxonomy to work in reverse to develop an overall solution and its several procedural steps can be developed. Therefore, the thesis presents an alternative method to HTA under which a problem can be better highlighted, to point out where aircrew cognitive failure can occur and to provide the necessary resources to minimize the potential problem aspect. 
An alternative method can be developed from the taxonomic framework and decision-making models explained in Chapter 2.2. This taxonomy can also be utilized to provide a constructive role or design solutions to mitigate issues and systematically designate scenarios that can lead to aircrew error. The step by step process can be utilized to observe and understand where pilot action may lead to an accident and design a solution to tackle the potential issue. In the case of a DVE, a pilot has lack of information due to low visibility (brownout, whiteout, night flight, fog, etc.) and being able to fill this void with the required virtual symbology can build the foundation to successful flight operation. With the addition of new information in the scope of the work, the validity of the information presented can be determined via its ability to help a pilot diagnose an event, if the pilot is not able to effectively diagnose an issue then the information step will be revisited. If an appropriate diagnosis is performed, the pilot will go on to set a goal required to complete an objective.

An error taxonomy analysis can be utilized to set the appropriate goals for accomplishing an objective, to see and validate if any goal is relevant to achieving the final objective. The validity of the goal can be judged based on the strategy and procedure of achieving the goal. The taxonomy can be utilized iteratively to provide the necessary developmental goals and to validate along the process about the sustainability and use of the implementation. The overall information processing, decision making and taxonomic models in cohesion can inform where aircrew could have failed cognitively and what can be done to not repeat it. The taxonomic framework is shown in Figure 36. New information can be presented to the pilot and the information can successfully be validated through a process to check the ability to diagnose the changing situations, setting goals, strategy setting, and procedure, action implementation. The development of the 2D, 3D symbology is thus derived using a taxonomic framework to produce the necessary cues that pilots would require to operate in DVE. In Figure 36 it can be seen that elements of the taxonomic framework correlate with the nodes on the combined information processing and decision-making model. 


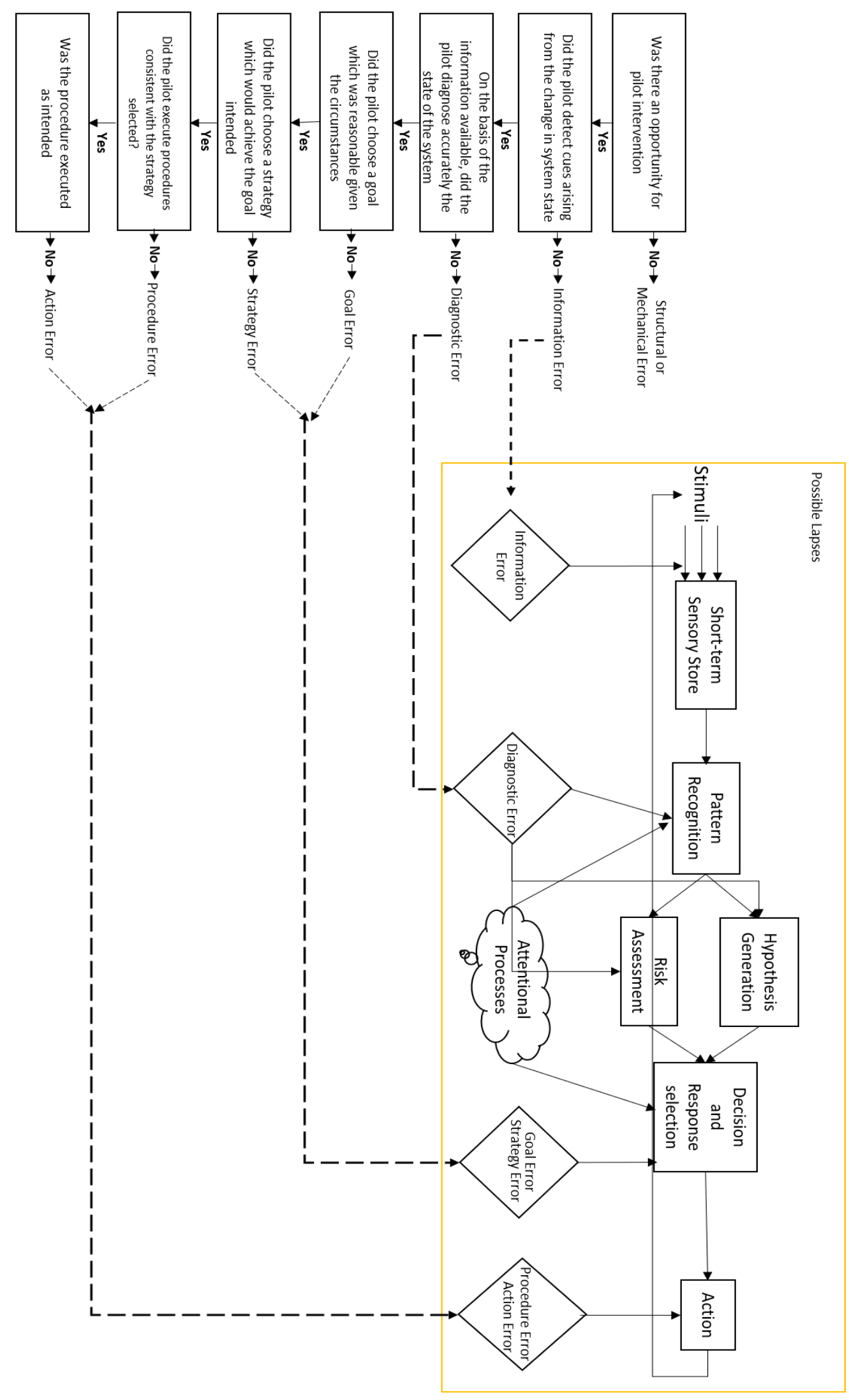

FIGURE 36: COMBINED MODEL OF TAXONOMIC AIRCREW ERROR AND DECISION-MAKING MODEL. 


\subsection{EVALUATION METHODS}

Development of new symbology is aimed to provide advanced capabilities to pilots. As an end user, a pilot will use highly capable and safety-oriented system to better serve their mission purpose. Evaluating how the developments actually improve pilot's performance is measured through non-intrusive questionnaires. Excessively low workload has been associated with a lack of attention leading to higher error rates, frustration, boredom, fatigue and lack of awareness with drifting attention and complacency. High workload environments cause pilots to rush their objectives, commit errors, ignore information, be frustrated, fatigued, and lack in effectiveness due to overburdened scenarios. Conditions such as workload, stress, and performance experienced using new symbology can be evaluated to understand usability and how they improve pilot operability. The questionnaire method of evaluation is based on pilot opinion in a simulated environment without the use of any intrusive devices.

Intrusive devices such as monitoring systems for heart rate, Blood Pressure, Electrocardiogram (ECG) and Electromyography (EMG) can be used to test body performance[33, 42, 58]. Using intrusive devices researchers can correlate quantitatively and qualitatively how well the human body performs and reacts to situations[57]. Utilizing evaluation methods rated on pilot opinion and experience provides qualitative results on the adaptability and usability of the displays. The combination of opinion-based evaluations and intrusive devices provide a greater understanding of the meaning behind the measurements. However, subjective measures are low cost, immediate with minimal post processing and non-intrusive. Intrusive testing costs are high and require immense post-processing efforts to use data.

For thE research, four different evaluation questionnaires are used. The tests serve to validate the findings of results of each other as they are overlapping with certain differences providing research to observe the improvement in implementing a visual cueing system for flight in DVE. The four rating scales are the Bedford scale, NASA TLX scale, Modified Cooper-Harper (MCH) and a generic opinion rating scale.

\section{BEFORD}

The Bedford scale was developed for the application in a flight environment and is based on the judgment of spare information processing capacity and workload and was developed by Roscoe, A.H. (scale shown in Figure 37)[59]. The Bedford scale is a subjective rating scale designed to identify pilot's spare mental capacity while completing a given task. The decisions are assessed using hierarchical decision making with a ten-point rating scale. Each point is assisted with a description of the associated level of workload. The Bedford method is considered simple and does not contain diagnostic capabilities. To complete the scale the pilot would explore the hierarchy narrowing down to the experienced rating by reading and utilizing the descriptions. The Bedford scale's capability to provide descriptions allows for a descriptor association to each numerical scale entity, allowing pilot interpretations [60, 61]. 
This method associates a description rather than a numerical value, whose scale might be different from subject to subject. The meaning of a numerical rating can be different for any one individual, providing a descriptor allows for a more accurate overall representation of opinion. A disadvantage with the use of the Bedford scale is the inability to obtain pilot feedback while performing the flight. The feedback would only be obtained once the flight is complete, this causes the results to be based on memory and may not convey what the pilot felt during a phase of flight. The numbers assigned on the scale are non-interval where the description varies on the pilot's response on the completion of the task. The scale arranges the difficulty of a task completion based on binary (Yes or No) objective completion. i.e. the rating or description for Level 3 does not represent half the workload of Level 6 numerically or descriptively. Based on the success or failure on the completion of a task, the pilot would rate the adequacy of workload that led to successful completion of an objective or failure [60]. The idea of spare capacity can be interpreted as where the operator has spare time, additional mental capacity to spare for the completion of another task at hand.

\section{Bedford Workload Scale}

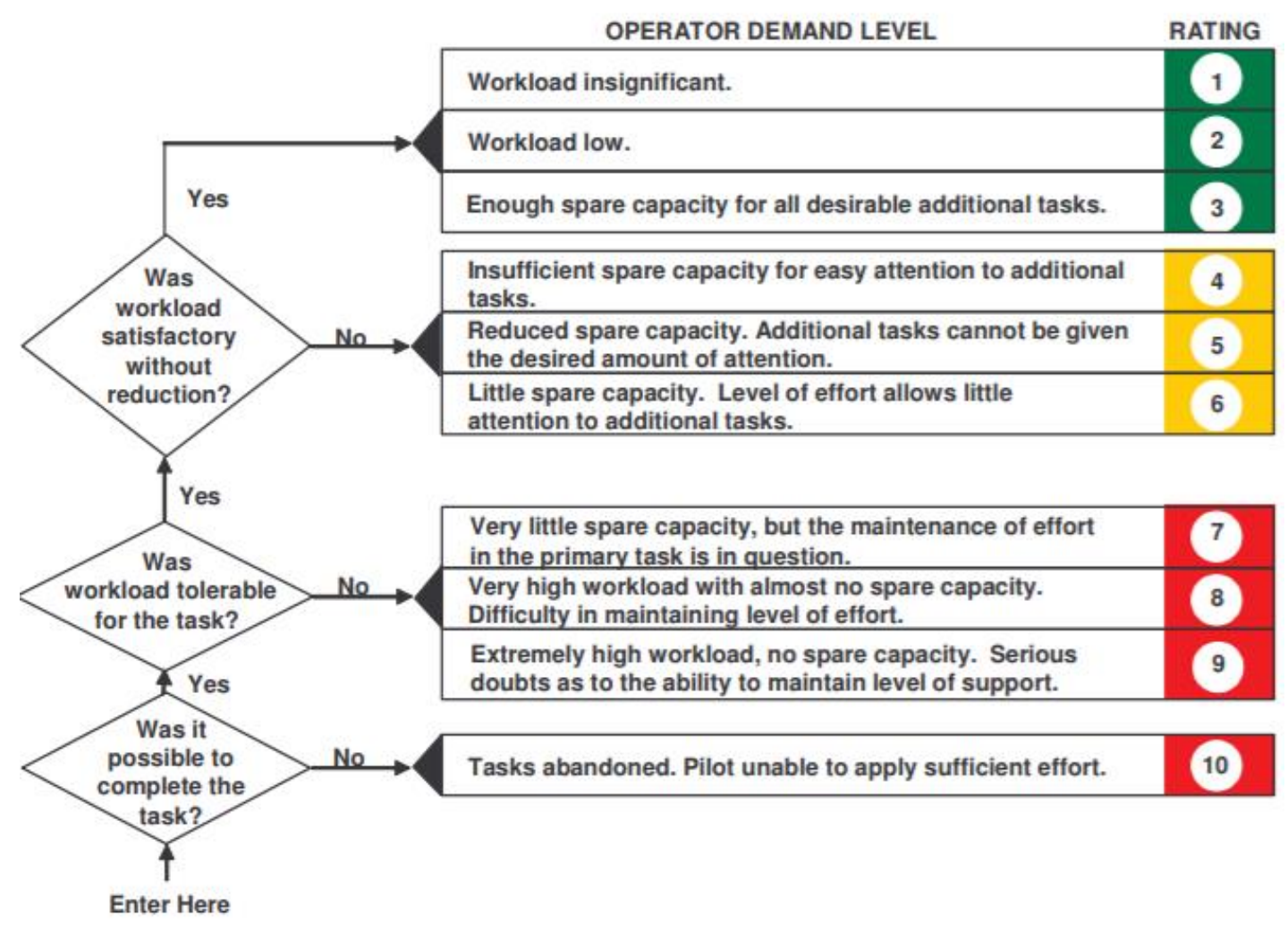

FIGURE 37: HIERARCHICAL DECISION TREE OF THE BEDFORD WORKLOAD SCALE [62]. 


\section{NASA TLX}

The NASA Task Load Index (TLX), shown in Figure 38 was developed by Hart and Staveland in 1988 is a multi-dimensional scale which rates the overall pilot workload and stress based on six major factors. The Mental, Physical, Temporal demands, Performance, Effort, and Frustration exerted for the completion of a task are collected [59].

The definition of each subscale is;

1. Mental demand (Low/High): How much mental and perceptual activity is required for thinking, deciding, calculating, and remembering or searching certain information that is being presented[60].

2. Physical Demand (Low/High): How much physical activity was required, pushing, pulling, turning, controlling activating, etc. to ideally plot to see whether a task was easy or strenuous [60].

3. Temporal Demand (Low/High): How much pressure does the operator feel due to the rate or pace at which the tasks or task elements occurred [60].

4. Performance (Good/poor): How successful was the operator in accomplishing the goals of a given task set by the experimenter [60].

5. Effort (Low/High): How hard did the operator have to work both mentally and physically to accomplish the level of performance[60].

6. Frustration level (Low/High): How insecure, discouraged, irritated stressed, and annoyed versus secure, gratified, content, relaxed, and complacent did the operator feel with the information presented [60].

The NASA TLX rating can be utilized as a subjective rating of an individual pilot to assess the applicability of symbology developments. Once a pilot has flown a certain mission profile, the index can be utilized to define subjectively how the pilot felt about the use of the symbology in terms of workload and stress. The mental, temporal, and physical demands are aspects of the workload, while performance, effort, and frustration levels can be associated with the level of stress. NASA TLX ratings do not provide an average of the pool of pilots, and the scale can mean different levels for different test subjects. 

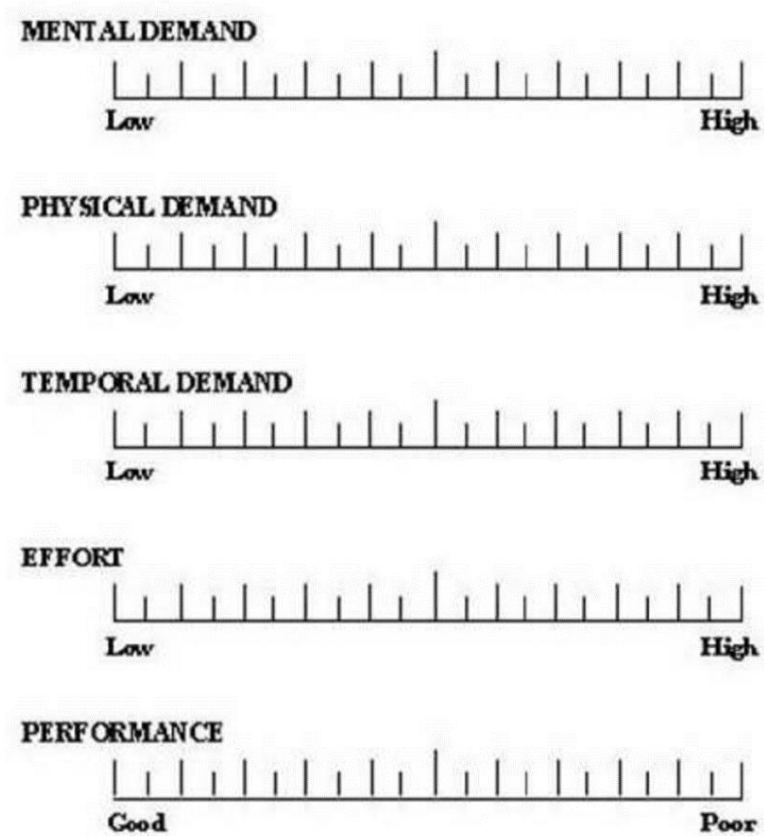

FRUSTRATION

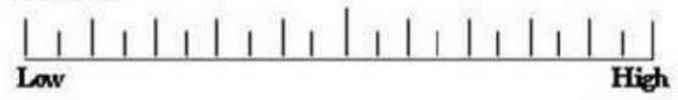

FIGURE 38: THE NASA-TLX INDEX FOR PILOT WORKLOAD AND STRESS BY HART AND STAVELAND IN 1988 [60]. MODIFIED COOPER-HARPER SCALE FOR DISPLAY EVLUATION

The Cooper-Harper scale helps researchers to evaluate aircraft handling qualities based on controllability, workload, and ability to attain adequate performance [61]. Like the Bedford rating scale, the Cooper- Harper scale follows a flowchart that helps analyze quantitatively and qualitatively the amount of workload or stress a pilot feels in the completion of a task. The pilots would follow the flowchart and choose which descriptions of aircraft characteristics and demands best represent their handling experience. The selection of appropriate descriptions on the scale associates a quantitative value between 1 and 10 . The original Cooper-Harper Scale has been used to test the handling qualities of an aircraft, and it has been modified to yield a similar use for assessing the UAV displays. Cummings, Myers, and Scott (2006) adapted the Cooper Harper scale to produce an assessment tool that can be used to assess the effectiveness and usability of a display system created for UAVs known as the Modified Cooper-Harper for Unmanned Vehicle Displays (MCH-UVD)(shown in Figure 39)[63]. MCH UVD is capable of judging the information acquisition, information analysis and decision-making elements of a four-stage information processing model proposed by Parasuraman et al. Displays which receive a rating between 1 and 2 means it was acceptable. Between 3 to 4 means there were deficiencies and warrants improvement. A rating between 5 and 8 means there are deficiencies and requires improvement. A rating of 9 or 10 means the display requires a mandatory redesign [63]. 


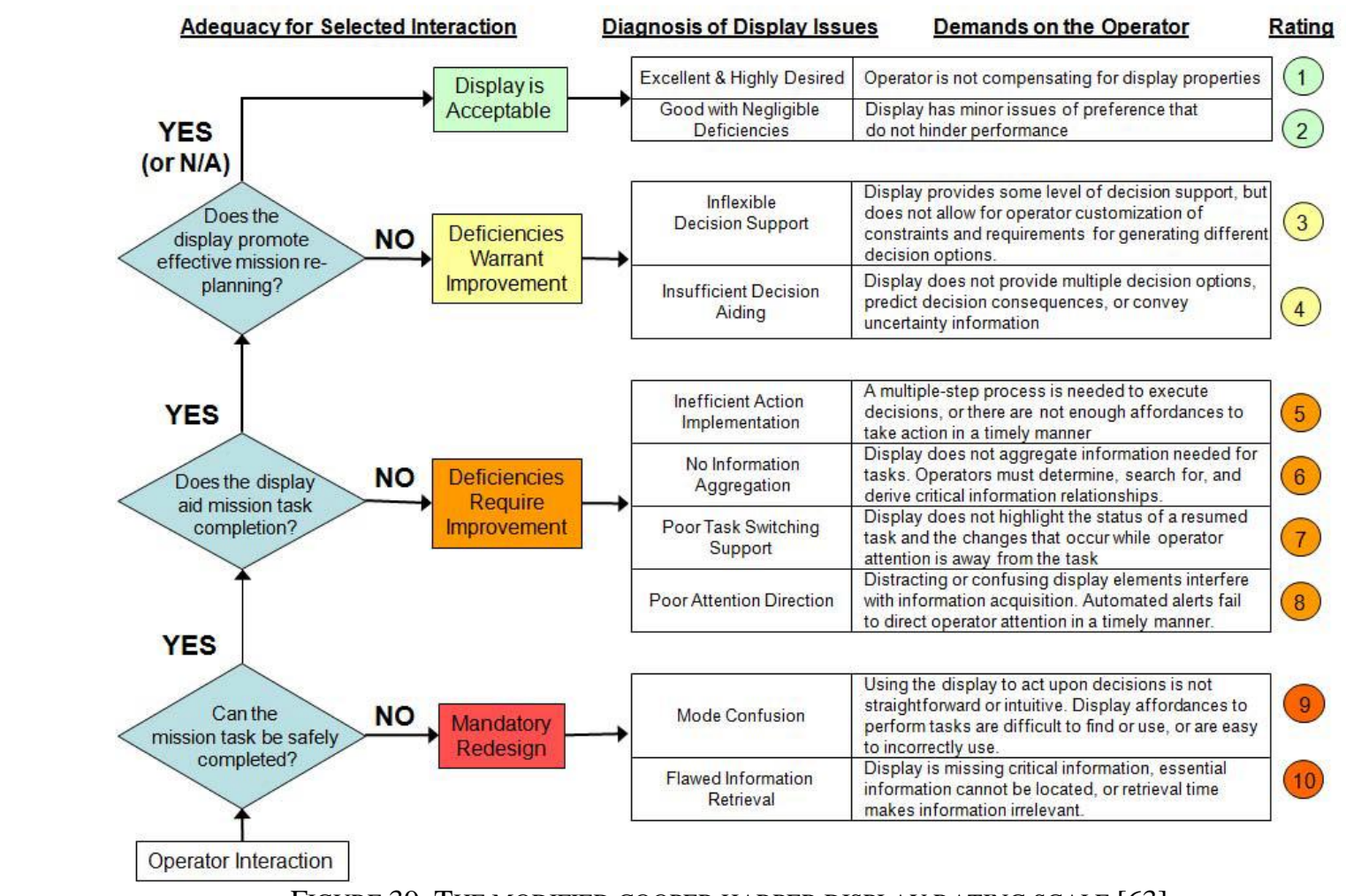

THE OPINION RATING SCALE

For the evaluation of textures present in the system, a different evaluation method had to be used due to a different testing standard and number of available participants. The Bedford Scale, NASA TLX, and MCH scale could not be used. The two available participants for the testing were asked to use a different satisfaction-based rating system. When evaluating a terrain texture, the rating system should encompass both pilot satisfaction for visual comfort and perceptibility of information. Thus, a different satisfactionbased scale must be used for the evaluation of perception. A satisfaction scale used in the medical field is utilized to obtain evaluations on participant satisfaction of visual comfort and perceptibility of information. The National Committee for Quality Assurance (NCQA) approved satisfaction survey for Health Plan Employer Data and Information Set is used to produce the necessary evaluation setup for textures. The satisfaction rating scale is a measure of a patient's satisfaction with the level of provided service towards a patient for medical needs and services [64].

The participant would be asked to attain and maintain a certain flight speed and altitude and asked to rate the texture between Bad (0), Poor (1), Fair (2), Good (3) and Excellent (4). The participant would have to rate the texture based on visual comfort and the perception of information from the texture. The participant would be asked to fly based on looking at the texture; once the flight condition is achieved, the actual altitude and speed are compared, a value between 0 and 5 is assigned. This rating system allows both the texture visual comfort and perceptibility to be evaluated. 


\section{CHAPTER 4.0 SYMBOLOGY DEVELOPMENT AND DESIGN}

The development of symbology was based on an iterative approach with initial developments being tested and changes made based on findings and pilot responses in preliminary flight tests. Thus, for the $2 \mathrm{D}$ symbology, 3D symbology and textures that were created, they were tested to observe validity in different unrecorded flight tests. There were no recorded data in the initial flight tests, the results were only pilot comments on the system at the point in time to warrant improvement and further development. This section outlines the design methodology and the preliminary results from initial testing and pilot feedback on developed aspects that led to the final symbology.

\subsection{SYMBOLOGY REQUIREMENTS BASED ON FLIGHT CONDITIONS}

3D symbology (virtual, conformal) is used to provide the necessary cues to a pilot about spatial awareness. When a pilot is in DVE, they must gain an understanding of the operational environment. The pilot needs information about the locality when landing in DVE. Pilots are not able to detect or perceive the drift of the helicopter, height above terrain, descent rate, ground speed, attitude, ground slope, terrain features, landing point location, obstacle clearance and identification of moving obstacles while trying to maintain a hover $[29,42]$. Visual cues are used to obtain a quick mental understanding of the listed information. Accurate ground speed and attitude can be represented via 2D orthographic symbology (system awareness). Virtual landing symbology and other terrain and spatial data can be represented via 3D symbology and other view modes to provide a different perspective to the pilot. 2D symbology cannot represent the spatial understanding; it is task-oriented and does not provide necessary visual cues that pilots would extensively use. Studying the requirements pilots would need, and existing 3D symbology sets like BOSS and Airbus provide necessary foundations for the development of the new conformal 3D cueing system. 2D Orthographic symbology similarly is derived based on existing systems, navigation and guidance improvements implemented.

\section{PILOT LOSS OF VISUAL CUES}

Pilots landing and approaching hover to require the cues indicating drift, ground slope, terrain features, landing point location, obstacle clearance and moving obstacle detection, and landing direction [29, 42].

\section{Drift:}

Helicopter pilots operate over several flight regimes, loosely classified to be low altitude (landing/hovering), climb/descent altitude and high altitude (cruise). When landing, and maintaining a low or high hover, drift is a crucial cue. Pilots would use an external cue to perceive the drift of the helicopter relatively. In high hover, a tower, trees, light poles etc. in a 
low hover, micro-textures are used for drift perception. However, with no outside visuals present, the task is rather tough. Highlighting one of the design goals.

\section{Ground slope:}

When a helicopter pilot is landing, they would justify the safety of a landing zone based on the slope gradient. If the slope gradient is larger than the operational limits of the helicopter, a dynamic rollover can occur on the lateral axis or a tail strike of the main rotor in the longitudinal axis. Pilots operating in DVE can also land in ditches, one skid on a top rock or simply too close to a large slope that leads to rotor strike with the ground.

\section{Terrain Features:}

In landing a helicopter, the longitudinal and lateral limit angles of the helicopter need to be accounted for when landing as this can lead to rollover or rotor strike. Thus, small stones or minimally elevated rocks can be devastating for landing as they can initiate rollovers. Larger terrain features, such as ditches, etc. near the landing zone are also of great importance.

\section{Landing Point Location:}

In the hover over a landing zone, the helicopter needs to land on a point. There should be no lateral or longitudinal drift. Helicopter pilots generally use windows at the bottom to view the exact point of touch down of a helicopter, however with blowing dust or snow or night operations without a spotlight; it is not possible. In prepared landing zones for a helicopter, the zone is marked by a large letter ' $\mathrm{H}$ '.

\section{Obstacle Clearance/Moving obstacle detection:}

Operating pilots need to be situationally aware of all obstacles, whether static or dynamic in a landing zone. When low altitude maneuvers are required, the placement and SA of what is around the pilot is critical.

\section{Landing direction}

Pilots tend to land in the direction of the wind. However, pilots choose to perform landings and takeoff in any direction they choose based on prevailing conditions. Pilots can be operating in low altitude scenarios, restricting specific approaches and extraction routes. Thus, a clear indication of approach direction or guidance/navigation is imperative.

Pilots use the environment to retrieve the cues specified in points 1 through 6 as a part of landing and takeoff operations in clear VMC conditions. A pilot who is not situationally aware of the above cue characteristics can make catastrophic combination of incorrect diagnosis, goal setting, strategy, and procedure engagements. In DVE a pilot is not supplemented with such visual cues. Thus, they can be provided virtually to provide the pilot with a necessary indication of a LiDAR identified landing zone. The pilot would use the virtually presented cues to land or takeoff. 


\subsection{HELICOPTER REFERENCED INFORMATION}

The developed 2D orthographic symbology is overlaid in cohesion with the 3D symbology to provide pilots with required information of the helicopter's performance, navigation, and control. 2D symbology provides redundancy and understanding to the pilot on how the helicopter relates to the environment. 2D symbology acts as the system awareness tool, providing cues on how the system is interacting with the spatial environment. 3D symbology is developed as a virtual representation of the environment to provide a pilot with VMC cues after inadvertently entering IMC. The requirement of elements of the orthographic representation is developed on existing standards. Previous works performed and with adding a level of redundancy to the information being retrieved from the 3D symbology. 2D symbology also acts profoundly in representing task-based information. The 2D symbology would be presented orthographically on the HDD while the $3 \mathrm{D}$ is represented in a conformal/perspective view.

TABLE 4: TABLE OF 2D ORTHOGRAPHIC DISPLAY GRAPHICS AND THEIR FUNCTION.

\begin{tabular}{|c|c|c|c|c|}
\hline Entity & $\begin{array}{l}\text { Cruise } \\
\text { or } \\
\text { Hover }\end{array}$ & Explanation & Reason & $\begin{array}{c}\text { Reference } \\
\text { Image }\end{array}$ \\
\hline Torque & Both & $\begin{array}{l}\text { A graphical representation of the } \\
\text { mast torque, showing a trend, for } \\
\text { instantaneous torque a digital } \\
\text { readout is provided. }\end{array}$ & $\begin{array}{l}\text { Engine torque acts to show } \\
\text { the "throttle/thrust" of the } \\
\text { helicopter. }\end{array}$ & B \\
\hline $\begin{array}{l}\text { Compass } \\
\text { Rose }[35, \\
36]\end{array}$ & Both & $\begin{array}{l}\text { Aircraft heading tape provides a } \\
\text { sense of direction to the pilot. } \\
\text { Provides trend to the pilots. }\end{array}$ & $\begin{array}{l}\text { The scale is used as opposed } \\
\text { to just readouts, as pilots } \\
\text { require trend and rate } \\
\text { information of heading. }\end{array}$ & $\mathrm{H}$ \\
\hline $\begin{array}{c}\text { Current } \\
\text { Heading } \\
\text { Digits [35] }\end{array}$ & Both & $\begin{array}{l}\text { The digital readout presents an } \\
\text { instantaneous heading of the } \\
\text { helicopter. }\end{array}$ & $\begin{array}{l}\text { For an instantaneous reading } \\
\text { of heading. }\end{array}$ & $\mathrm{H}$ \\
\hline $\begin{array}{l}\text { Roll Pointer } \\
\qquad[36]\end{array}$ & Both & $\begin{array}{l}\text { Presents the roll tendency of the } \\
\text { helicopter, the scale provides a } \\
\text { trend. }\end{array}$ & $\begin{array}{l}\text { Provides trend and instant } \\
\text { numerical understanding of } \\
\text { roll. }\end{array}$ & $\mathrm{G}$ \\
\hline $\begin{array}{c}\text { Side Slip } \\
\text { Indicator } \\
{[36]}\end{array}$ & Hover & $\begin{array}{l}\text { Presents the pilot with an } \\
\text { indication as to whether the } \\
\text { helicopter is slipping or } \\
\text { skidding. Skidding if the ball is }\end{array}$ & $\begin{array}{l}\text { Helps keep the pilot in } \\
\text { coordinated flights, } \\
\text { understanding of } \\
\text { slipping/skidding in turning. }\end{array}$ & I \\
\hline
\end{tabular}


on the opposite side of the turn and slipping if on the same side of the turn.

Horizon Both The horizon line presents the

Line [35]

Lat/Lon

Acceleration

Cue

Lat/Lon

Velocity

Vector

Central

Aircraft

Marker

(CAM) [29,

36]

Altitude

Indicator

[35]

Vertical

Velocity

Indicator

[29]

Vertical

Acceleration

Cue [29]

position of the horizon to the pilot.

Hover Presents the instantaneous acceleration of the helicopter, shows where the velocity vector will eventually be.

Hover Represented via a line, velocity direction and magnitude are presented. CAM is used to scale and perceive the velocity.

Hover Serves as a reference scale for velocity vector.

Both Displays the digital readout of altitude. the helicopter, referenced with the CAM.

Both Presents the leading indicator for vertical velocity of the helicopter. velocity vector, RGS, vertical information. current altitude based on Radar altimeter and barometric

\section{Both Presents the vertical velocity of}

$\mathrm{O}$

Provides a reference to where the horizon is in flight.

$\mathrm{O}$

Provides the pilot an idea of where the aircraft is "accelerating" to. It is useful F in guidance, can be placed to in the $\mathrm{DH}$ to decelerate accurately to an SLZ.

Provides trend of the helicopter lateral and F longitudinal velocity.

Provides a reference for trend When $<600 \mathrm{ft}$ radar altitude is shown. When >600ft, D barometric altitude is shown.

Shows trend of rate of climb to pilot, trends help provide more situational awareness than instantaneous values.

Shows instantaneous acceleration in vertical $\mathrm{E}$ direction, helps with keeping steady hover. 


\begin{tabular}{|c|c|c|c|}
\hline $\begin{array}{c}\text { Air/ } \\
\text { Groundspeed } \\
{[35]}\end{array}$ & Both & $\begin{array}{l}\text { Presents the current ground and } \\
\text { airspeed, transitions between } \\
\text { ground speed and airspeed } \\
\text { measurements. }\end{array}$ & Provides the rate information \\
\hline $\begin{array}{c}\text { Rising } \\
\text { Ground } \\
\text { Symbol } \\
\text { (RGS) [35] }\end{array}$ & Hover & $\begin{array}{l}\text { Presents the position of the } \\
\text { ground relative to the helicopter, } \\
\text { is driven by the indicated } \\
\text { altitude. }\end{array}$ & $\begin{array}{l}\text { Represents the ground in } 2 \mathrm{D} \\
\text { symbology to reference with } \\
\text { CAM for landing, provides } \\
\text { rate and location. }\end{array}$ \\
\hline $\begin{array}{c}\text { Pitch Ladder } \\
\text { [36] }\end{array}$ & Cruise & $\begin{array}{l}\text { Presents the current aircraft } \\
\text { pitch of the helicopter, also } \\
\text { rotates to present roll of the } \\
\text { helicopter. }\end{array}$ & $\begin{array}{l}\text { Provides a rate and trend of } \\
\text { helicopter pitch and roll } \\
\text { relative to the horizon line }\end{array}$ \\
\hline $\begin{array}{l}\text { Dog House } \\
\text { LZ, (DH) } \\
{[8]}\end{array}$ & Hover & $\begin{array}{l}\text { Provides a geo-referenced } \\
\text { location of the landing zone. } \\
\text { CAM used to reference altitude } \\
\text { above ground. provides } \\
\text { guidance to LZ. }\end{array}$ & $\begin{array}{l}\text { Navigation cue in } 2 \mathrm{D} \\
\text { symbology to provide non- } \\
\text { VFR reference LZ. }\end{array}$ \\
\hline $\begin{array}{c}\text { Waypoint } \\
\text { Marker [57] }\end{array}$ & Both & $\begin{array}{l}\text { 2D waypoint marker embedded } \\
\text { in the Compass rose provides the } \\
\text { bearing guidance to waypoints. }\end{array}$ & $\begin{array}{l}\text { Provides directional bearing } \\
\text { cues in } 2 \mathrm{D} \text {, can be combined } \\
\text { with } \mathrm{DH} \text {. }\end{array}$ \\
\hline $\begin{array}{c}\text { Localizer } \\
{[30]}\end{array}$ & Both & $\begin{array}{l}\text { Provides guidance to the SLZ } \\
\text { once selected. Driven by bearing } \\
\text { deviation between helicopter } \\
\text { and SLZ. }\end{array}$ & $\begin{array}{l}\text { Provides correction in } \\
\text { heading maintenance for the } \\
\text { pilot. }\end{array}$ \\
\hline $\begin{array}{l}\text { Flight Path } \\
\text { Marker [65, } \\
\text { 66] }\end{array}$ & Both & $\begin{array}{l}\text { Presents the pilot with a } \\
\text { prediction of where the aircraft } \\
\text { will travel given direction of } \\
\text { travel. }\end{array}$ & $\begin{array}{l}\text { Navigation cue in } 2 \mathrm{D} \\
\text { symbology provides the } \\
\text { location of where the } \\
\text { helicopter fly to. }\end{array}$ \\
\hline
\end{tabular}

The hover phase symbology is composed of many individual components which are referenced on a central scale, known as the Central Aircraft Marker (CAM) (Refer to Table 4 and Figure 66 for reference). The CAM acts as a scale from which pilots can reference other components such as Vertical velocity indicator (E in Figure 66), velocity vector(F in Figure 66), acceleration cue, RGS(A in Figure 66), and LZ marker(A in Figure 66). The pilot refers to the scale, depending on what information they are looking for. The RGS 
symbol is referred to the CAM, such that each set of ticks represents 25 feet off the ground for the RGS. Similar to the RGS, the LZ marker follows the same scaling to the CAM. The velocity vector is referred to as 5 knots for each tick mark of the CAM. The vertical velocity indicator is referenced to the CAM such that each tick represents $100 \mathrm{ft} / \mathrm{min}$ rate of climb. Data conversion was used to represent information to the pilot, converting from SI to the meteorology aviation maritime unit system (MAM) and conversion of coterminal angles that pilots would easily understand (Ex. -90 deg is 270 deg bearing). The sideslip angle represents aircraft slip (shown in Figure 42), in a coordinated turn, the ball stays in the center. When slipping the ball moves towards the direction of the slip and while skidding it moves in the opposite direction of the turn.

The flight path marker is used to show where the helicopter will fly based on the climb angle and track or slip of the aircraft. It essentially shows where the aircraft will fly to. The flight path angle is calculated using Equation 2.1 and is visualized in Figure 40.

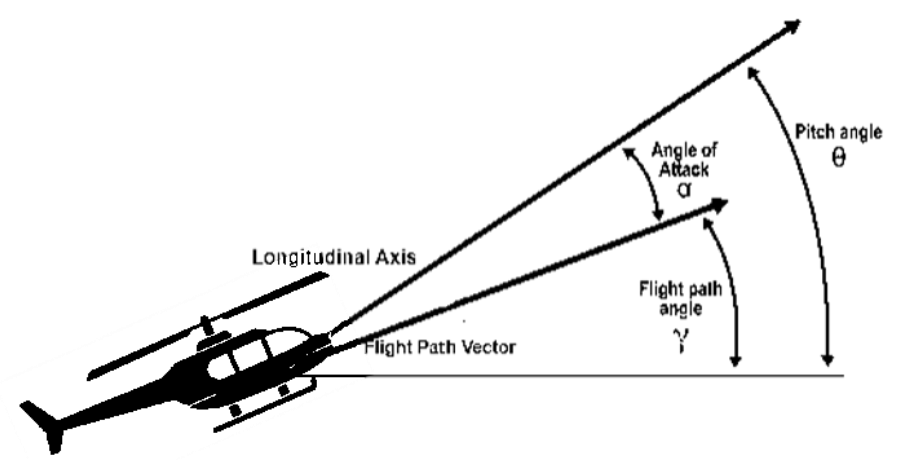

FIGURE 40: ILLUSTRATION OF ANGLE OF ATTACK, FLIGHT PATH ANGLE AND PITCH ANGLE DERIVATION ANGLES OF ROTORCRAFT FLIGHT [67].

$$
\gamma=\tan ^{-1}\left[\frac{-V_{D E}}{V_{G R}}\right] \ldots E q 2.1[68]
$$

$\boldsymbol{V}_{\boldsymbol{G} \boldsymbol{R}}$ is the Ground speed of the rotorcraft.

$-V_{D E}$ is the vertical speed of the rotorcraft

The calculated ground speed and vertical speed yield the resultant flight path of the aircraft which can be approximated to see where they end up. 


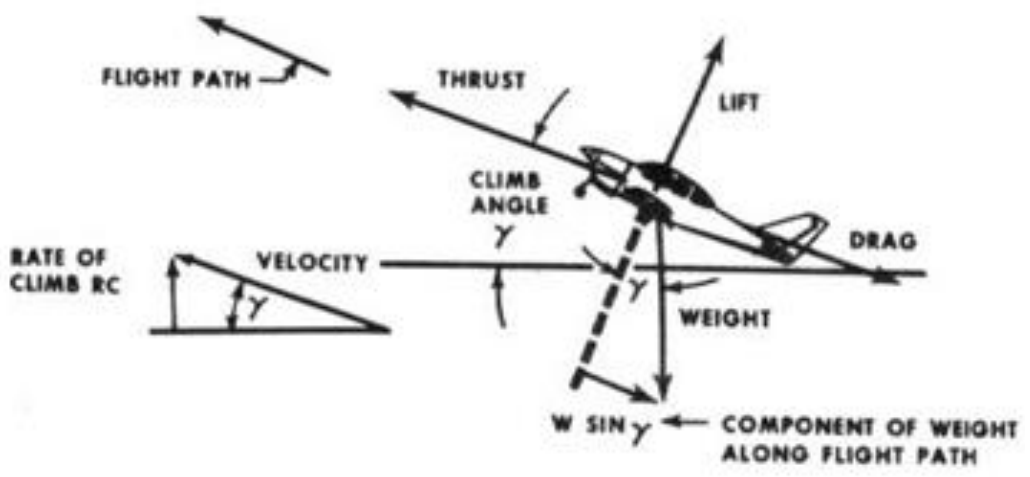

FIGURE 41: ILLUSTRATION OF FORCES ON AIRCRAFT [69].

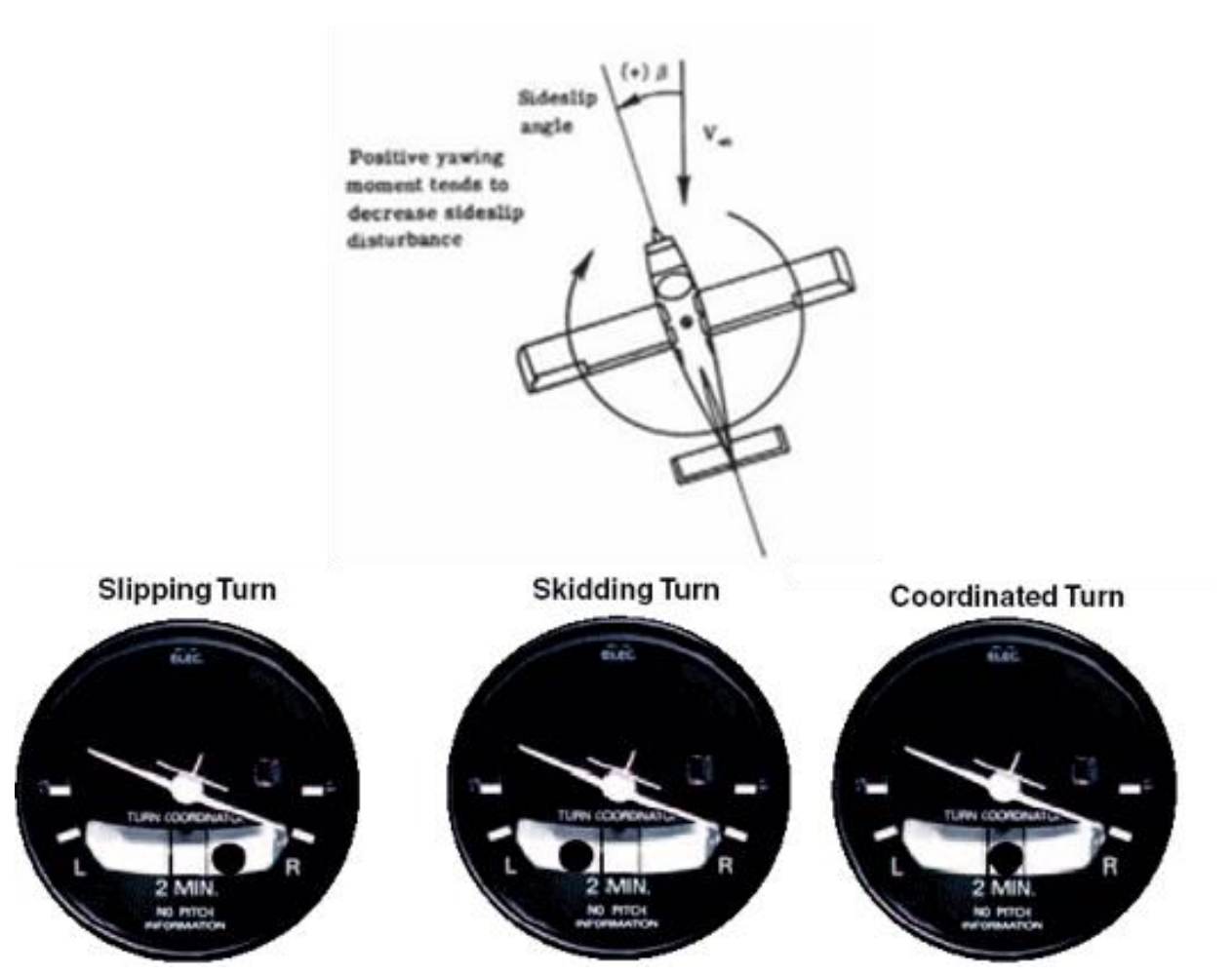

FIGURE 42: SIDESLIP/SLIP INDICATOR OF AN AIRCRAFT AND ITS DERIVATION [70, 71].

$$
\begin{gathered}
\boldsymbol{\beta}=\tan ^{-1}\left[\frac{\boldsymbol{V}_{\boldsymbol{B}}}{\left(\boldsymbol{V}_{\boldsymbol{B}}^{\mathbf{2}}+\boldsymbol{W}_{\boldsymbol{B}}^{\mathbf{2}}\right)^{\frac{1}{2}}}\right] \text { or } \boldsymbol{\beta}=\sin ^{-1}\left[\frac{\boldsymbol{V}_{\boldsymbol{B}}}{\boldsymbol{V}_{\text {Tas }}}\right] \ldots E q 2.2 \text { [68] } \\
\boldsymbol{V}_{\text {Tas }} \text { is the True Airspeed of the rotorcraft. }
\end{gathered}
$$

$\boldsymbol{V}_{\boldsymbol{B}}$ is the rotorcraft velocity in the body axis. (Lateral direction)

The calculated velocity of the rotorcraft in the body axis and the true airspeed of the aircraft yields the sideslip angle of the helicopter, which represents the slip of the aircraft in turns. 


\subsection{DESIGN OF SYMBOLOGY BASED ON ERROR TAXONOMY}

As described in Chapter 2.2, the Aircrew error taxonomic framework, along with the models of human information processing and decision making, can be utilized to develop the solutions necessary to prevent pilot-induced accidents. The taxonomic framework is a seven-tier process that deduces where a pilot can make a mistake based on information present and the situation at hand. Thus, given a situation where a system state change exists with a deficient/limiting visibility at play (such as a dust/snow cloud), an immediate solution can be introduced to solve the risen issue. The following set of flowcharts describe the possible solution which would be introduced to solve each cue pilots need when they inadvertently enter IMC.

A vertical dynamic height marker that correlates and traverses vertically with respect to the helicopter has been implemented and is placed in the pilot's FOV such that motion of the helicopter in the vertical direction be understood. As an orthographic representation, the radar altimeter and vertical velocity components are present in the 2D symbology to supplement the pilot instantaneous understanding of altitude drift. Figure 43 shows the process implemented with the Taxonomic Aircrew Framework to create the necessary solution to lacking altitude drift information.

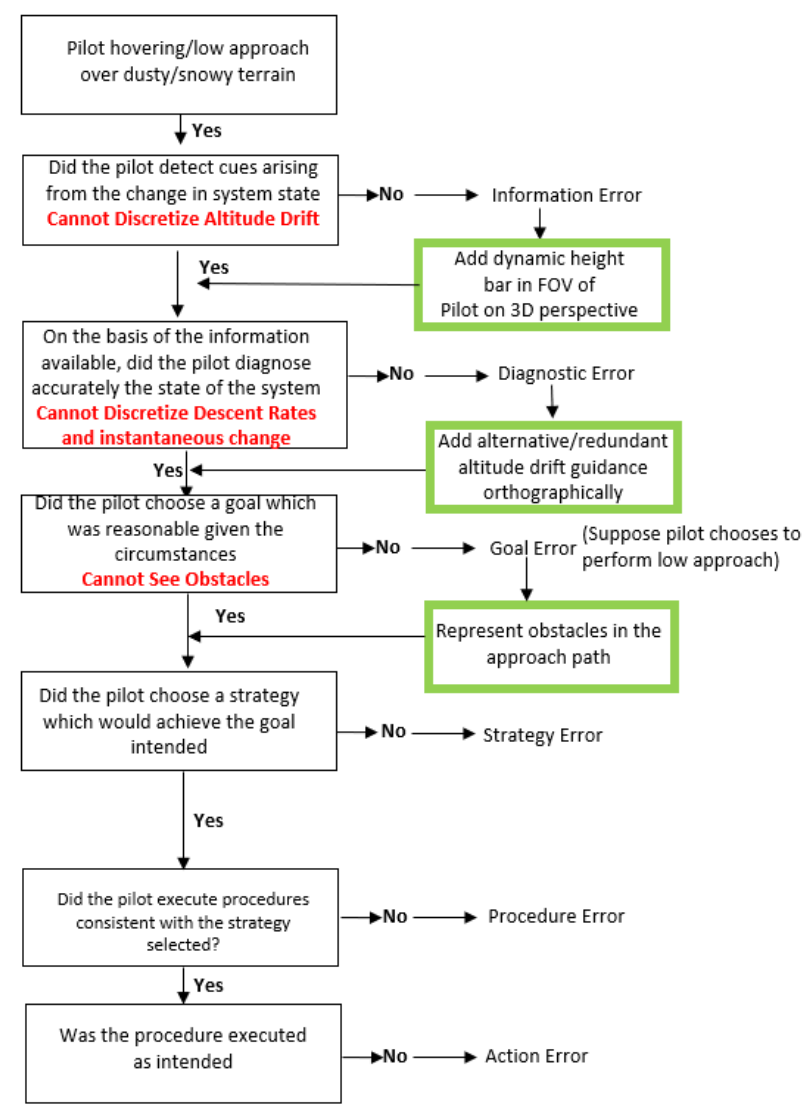

FIGURE 43: THE TAXONOMIC AIRCREW ERROR FRAMEWORK USED TO PROVIDE SOLUTION TO INABILITY IN DISCRETIZING ALTITUDE DRIFT. 
Similarly, to provide an understanding of the lateral and longitudinal drift cue, the concept of Relative Motion was replicated. When stopped at a traffic light, the motion of the one's vehicle can confuse that their neighbor's car is moving or vise-Versa. The implementation of this concept via the placement of two stationary structures (Pillars) in the view of the pilots helps perceive their motion with respect to the columns in front of them. The two stationary structures replicate trees or light poles that pilots would use in nature in more clear VFR conditions to help them judge drift. The two pillars would be placed at 45 degrees from the center. In the orthographic 2D symbology, the velocity vector which traverses in the longitudinal and lateral directions helps the pilot understand minor motions when the aircraft is too close to observe and deduce primary, such that they are present in the peripheral and do not obstruct other LiDAR information. In addition, a top-down view mode is added such that pilots can determine their position and minor motions of the helicopter with respect to the currently selected landing zone. Figure 44 highlights the depicts the use of the Taxonomic framework for introduced solution to discretize lateral drift.

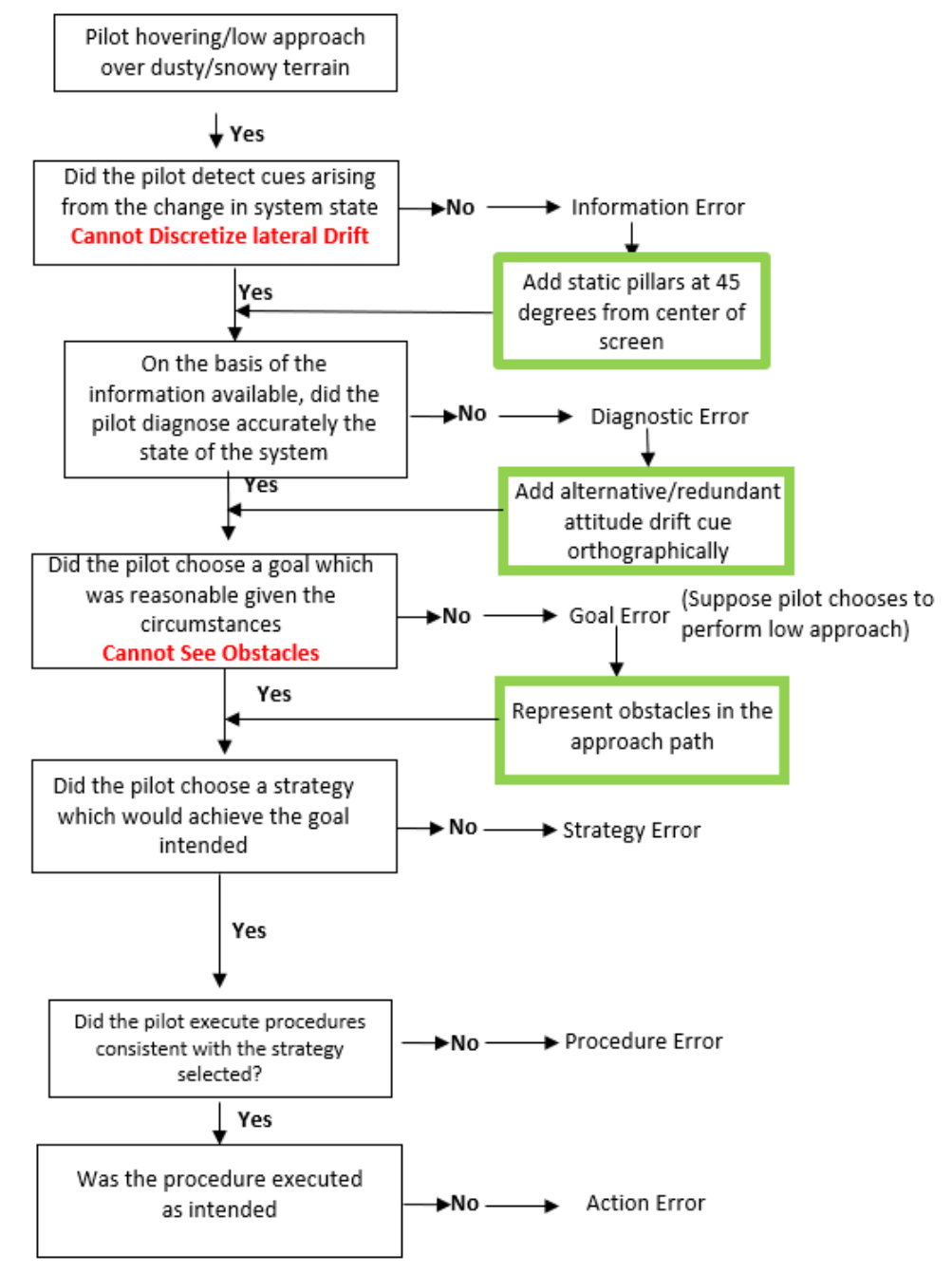

FIGURE 44: THE TAXONOMIC AIRCREW ERROR FRAMEWORK USED TO PROVIDE SOLUTION TO LACK OF LATERAL DRIFT CUE. 
The DVENS system scans a FOV and ranges and presents safe zones. Within this zone, the pilot can select a point of interest to land. When a point is located, the pilot would want to land there. A traditionally used symbol " $\mathrm{H}$ " is used to signify the center of the selected landing point. A triangle would be added on top of the 'H'; the pilot would land the helicopter skids at the base of the triangle. The tip of the triangle can be used to orient themselves and acts as a foreshortening cue. In the orthographic symbology, the LZ marker is used to represent the LZ. Once a pilot selects a landing zone, the LZ marker is presented in 2D symbology and is used to decelerate to the LZ by placing the acceleration cue at its center. Figure 45 highlights the depicts the use of the Taxonomic framework for introduced solution to discretize longitudinal drift.

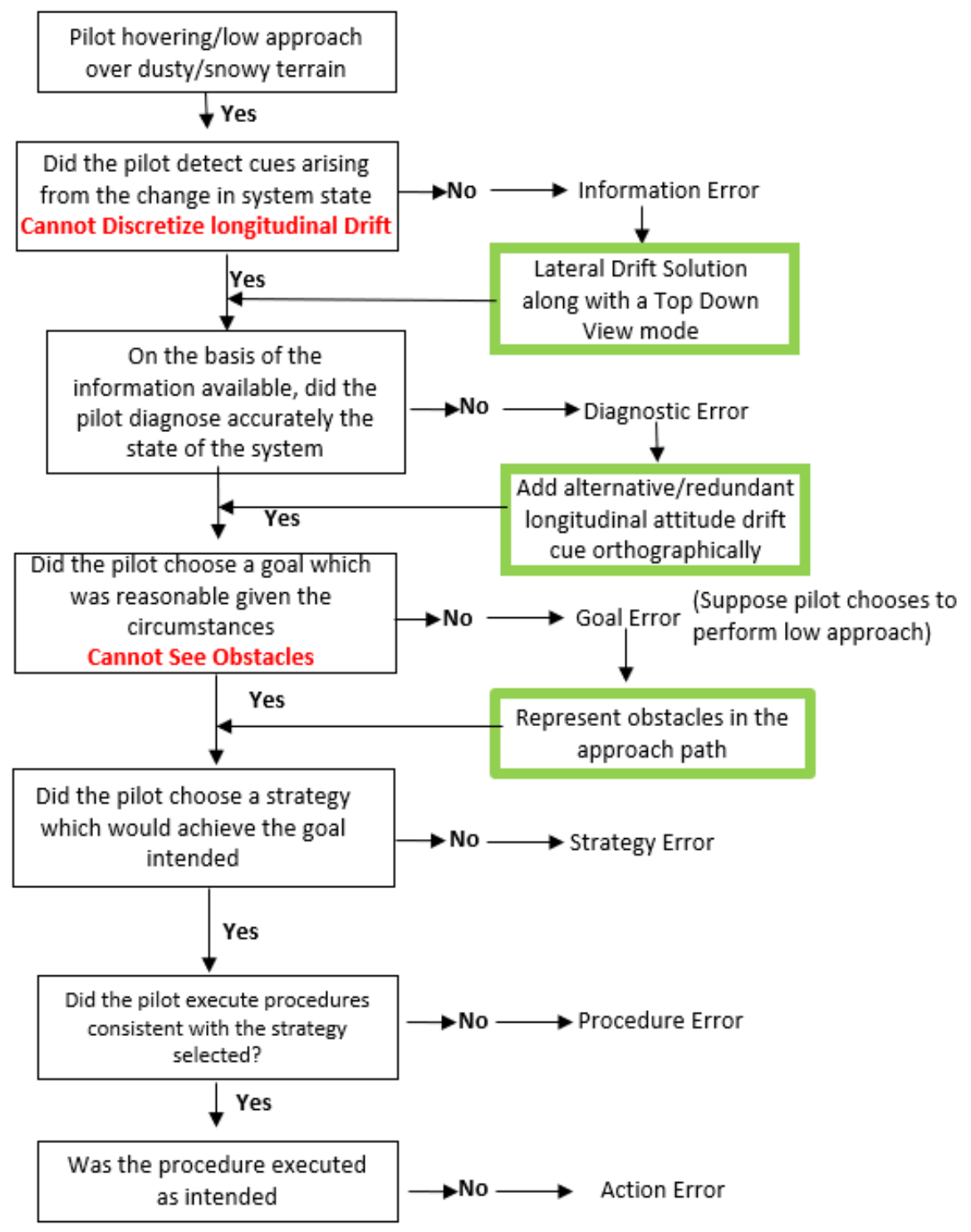

FIGURE 45: THE TAXONOMIC AIRCREW ERROR FRAMEWORK USED TO PROVIDE SOLUTION TO INABILITY IN DISCRETIZING LONGITUDINAL DRIFT. 
The representation of navigation information has been implemented at multiple levels to aid pilots. Pilots are provided with LZ orientation. Pilots would be allowed to change the orientation of the 3D symbology to fit their approach direction and make changes as required. There are limited navigation cues in 3D symbology except for 3D waypoint markers. There are more dedicated resources in 2D orthographic symbology. A replicated ILS method is used to provide bearing deviation and altitude deviation indication between the aircraft and LZ. A waypoint marker embedded in the compass rose provides the bearing deviation between waypoints to help navigate the pilot through a course. When in landing, once a zone is selected, the LZ marker is highlighted to show the position of the landing zone, the pilot can place the acceleration cue in the middle of the marker to decelerate to the LZ. In the meantime, the pilot can use the ILS system to position themselves on the course and descent rate. Figure 47 highlights the depicts the use of the Taxonomic framework for introduced solution to discretize landing point.

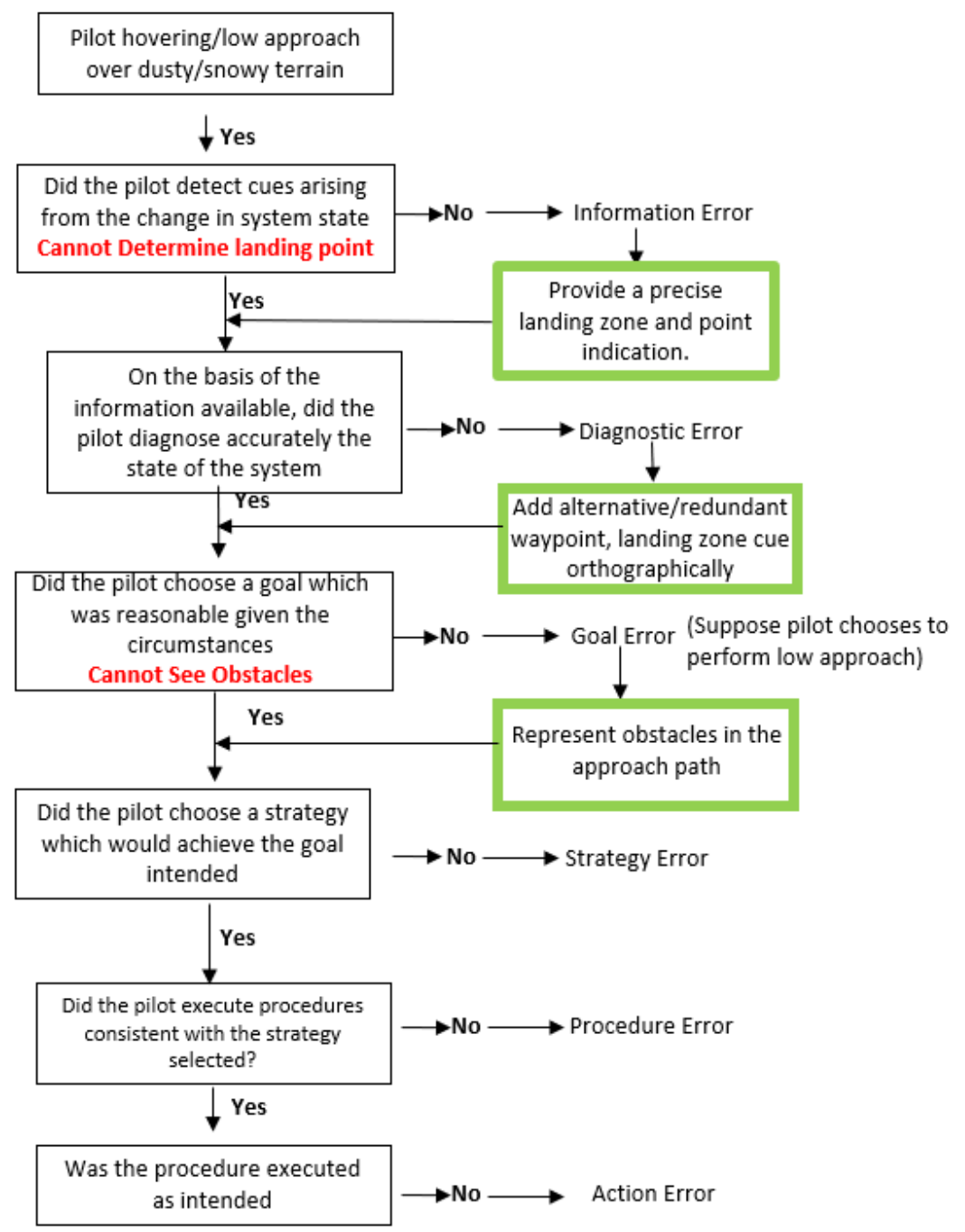

FIGURE 46: THE TAXONOMIC AIRCREW ERROR FRAMEWORK USED TO PROVIDE SOLUTION TO INABILITY IN DISCRETIZE LANDING POINT LOCATION. 
The representation of terrain information, to give a perception of altitude and slope while in flight, hover, landing or takeoff can be better augmented and represented via the use of terrain textures. The use of texture to show surface attitude have been extensively covered in Chapter 2.5.3. The secondary or top-down view mode also presents a greyscale color palette that shows pilots the elevation changes of the terrain around them, lighter meaning higher elevation and darker meaning lower elevation. Figure 47 highlights the depicts the use of the Taxonomic framework for introduced solution to discretize terrain information.

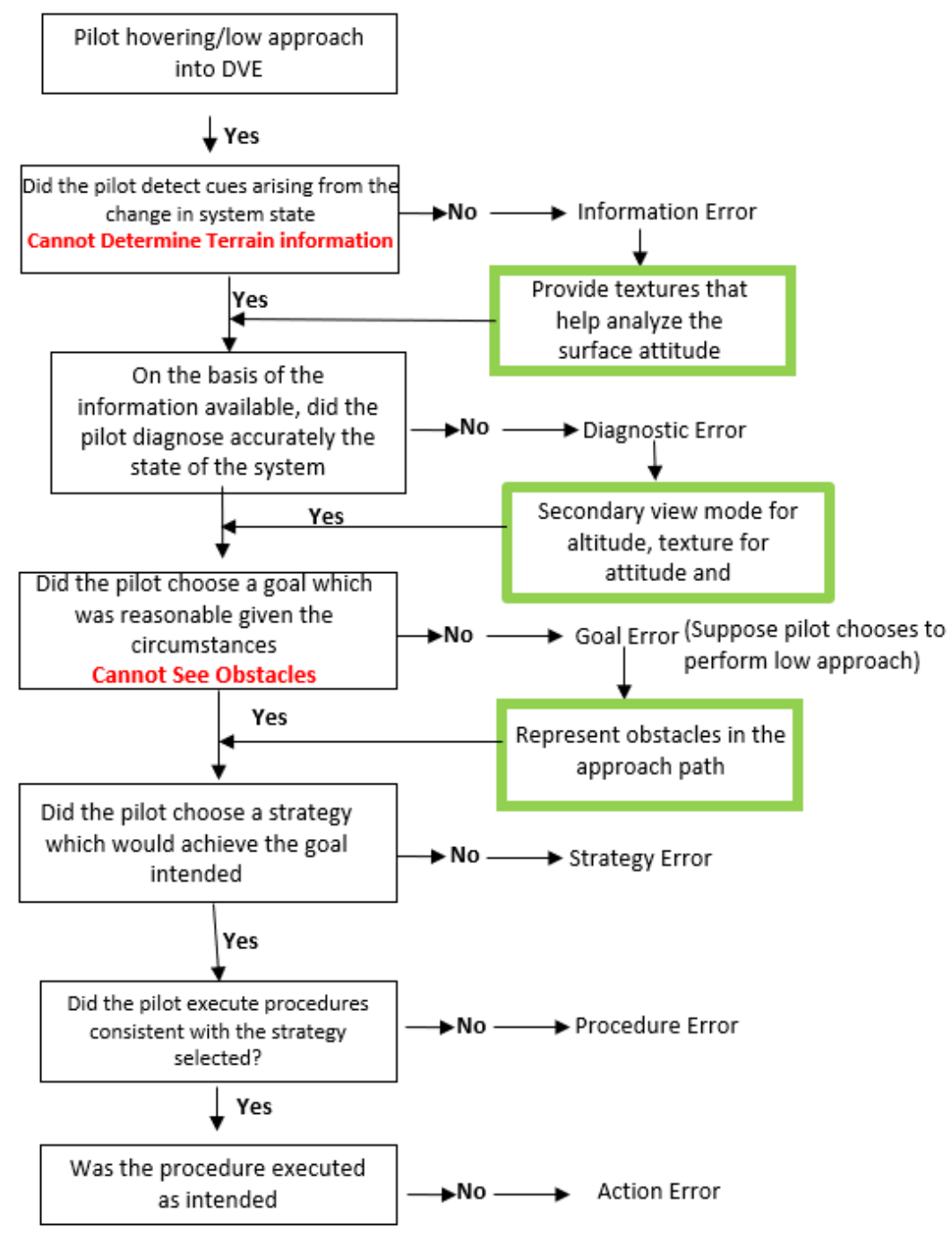

FIGURE 47: THE TAXONOMIC AIRCREW ERROR FRAMEWORK USED TO PROVIDE SOLUTION TO INABILITY IN DISCRETIZE TERRAIN INFORMATION.

When the pilots choose the landing zone and point, they can land in the area; however, there can be obstacles. Thus, obstacle representation can be used to show the pilot where the exact obstacles exist in relation to the 
helicopter. The obstacles such as buildings, ditches, trees, etc. can all be represented, however the pilot decision to land in the area may not always be correct. The idea of decision making, and execution plays a big role in this aspect. Given mission circumstances, the pilot may or may not make the best decision, to provide them the correct amount of information to make sound decisions is key. In addition to showcasing static and dynamics obstacles to the pilots, an 'operating perimeter' can be greatly helpful, to signify their maneuvering limits in the landing zone. When pilots land in obstacle dense areas such as cities or urban environments, they would use rotor spacing to situate the helicopter from the obstacles. Rings which signify rotor diameter can be placed at ground level to show how far from the closest obstacle the helicopter rotors are. Such information would be provided in the forward view and top-down view. A safety factor approach can be used to show the rings at two times the rotor diameter such that pilots are well aware of the tolerances and distance to the obstacles. Pilots land and takeoff into the wind, thus, symbology that shows them environmental wind conditions are represented via the auxiliary symbology. Figure 48 highlights the depicts the use of the Taxonomic framework for introduced solution to discretize approach direction. Figure 52 shows the rotor spacing cueing implementation in concept.

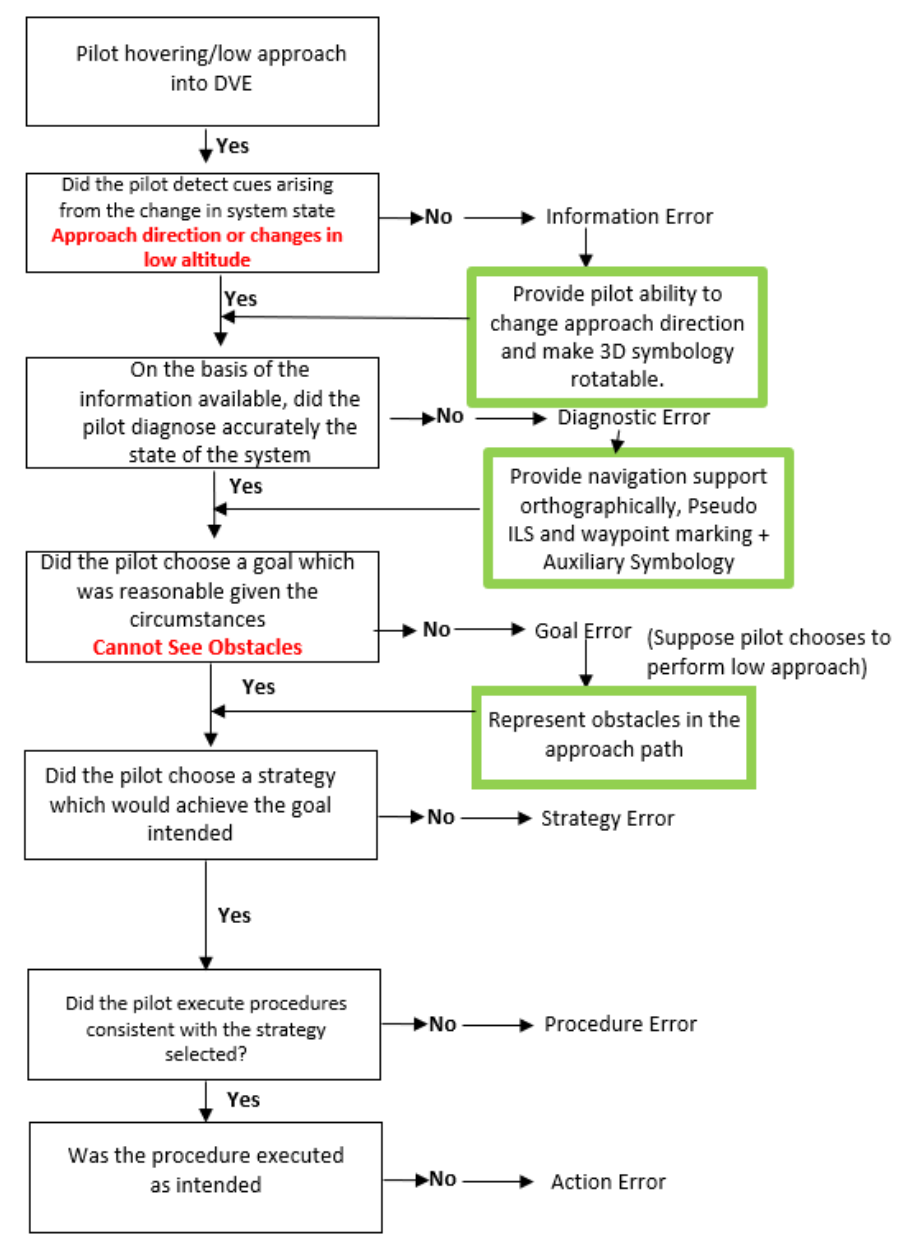

FIGURE 48: THE TAXONOMIC AIRCREW ERROR FRAMEWORK USED TO PROVIDE SOLUTION TO INABILITY IN DISCRETIZE APPROACH DIRECTION. 
Figure 49 shows the hover and cruise symbology respectively that was derived using the Taxonomic Framework and the literature review. Figure 51 shows the 3D symbology which was developed using the Aircrew Error Taxonomy. Figure 52 shows the complete display in deployed. Figure 53 is the shows the LiDAR detection, 2D symbology, 3D symbology in action.

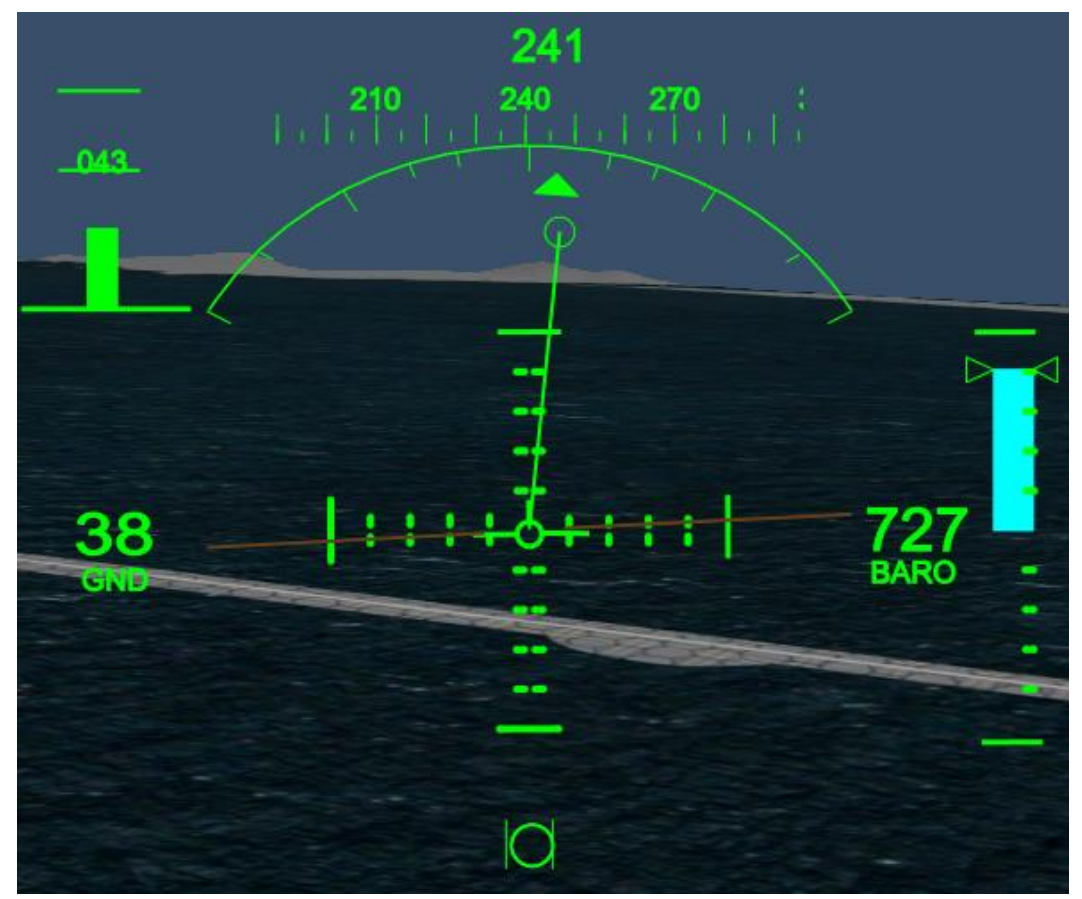

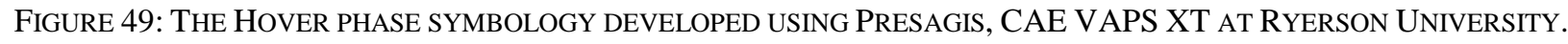

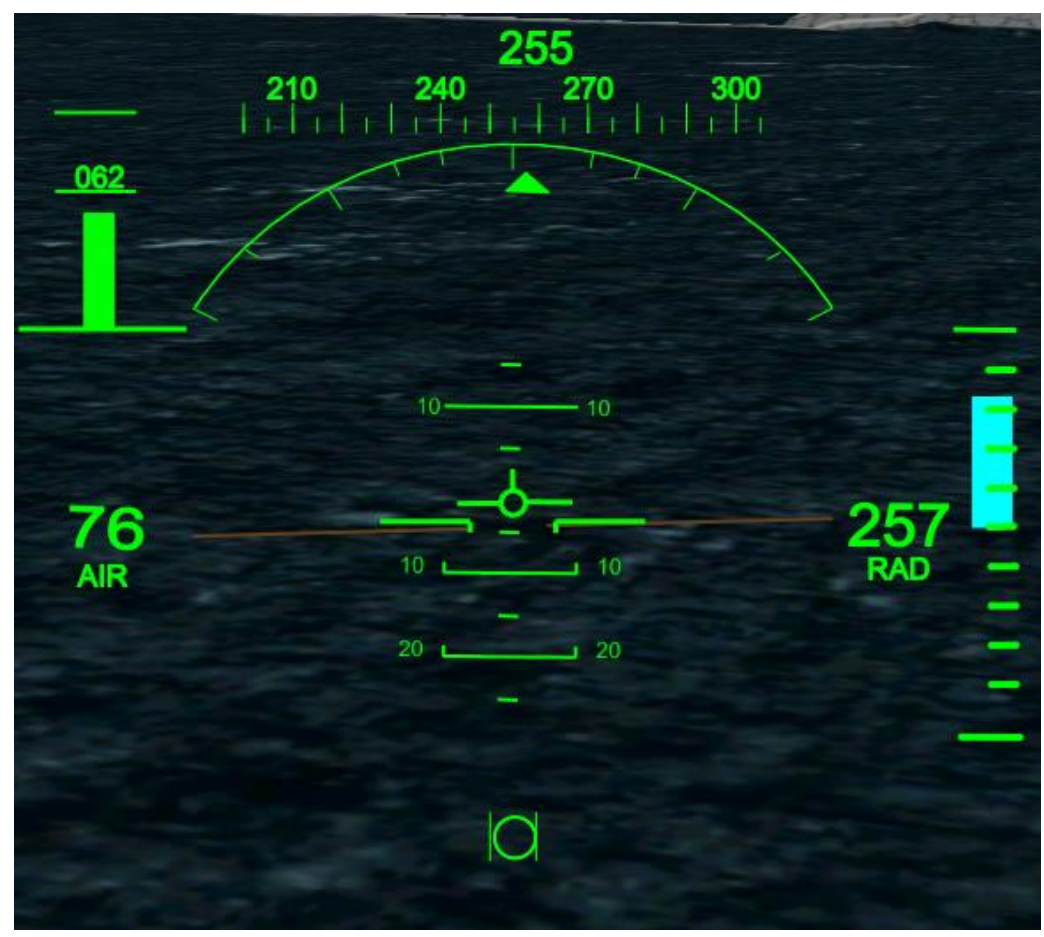


Figure 50: The CRUISE PHASE SYMBology DEVELOPED using PRESAgIS, CAE VAPS XT AT RYERSON UNIVERSITY.
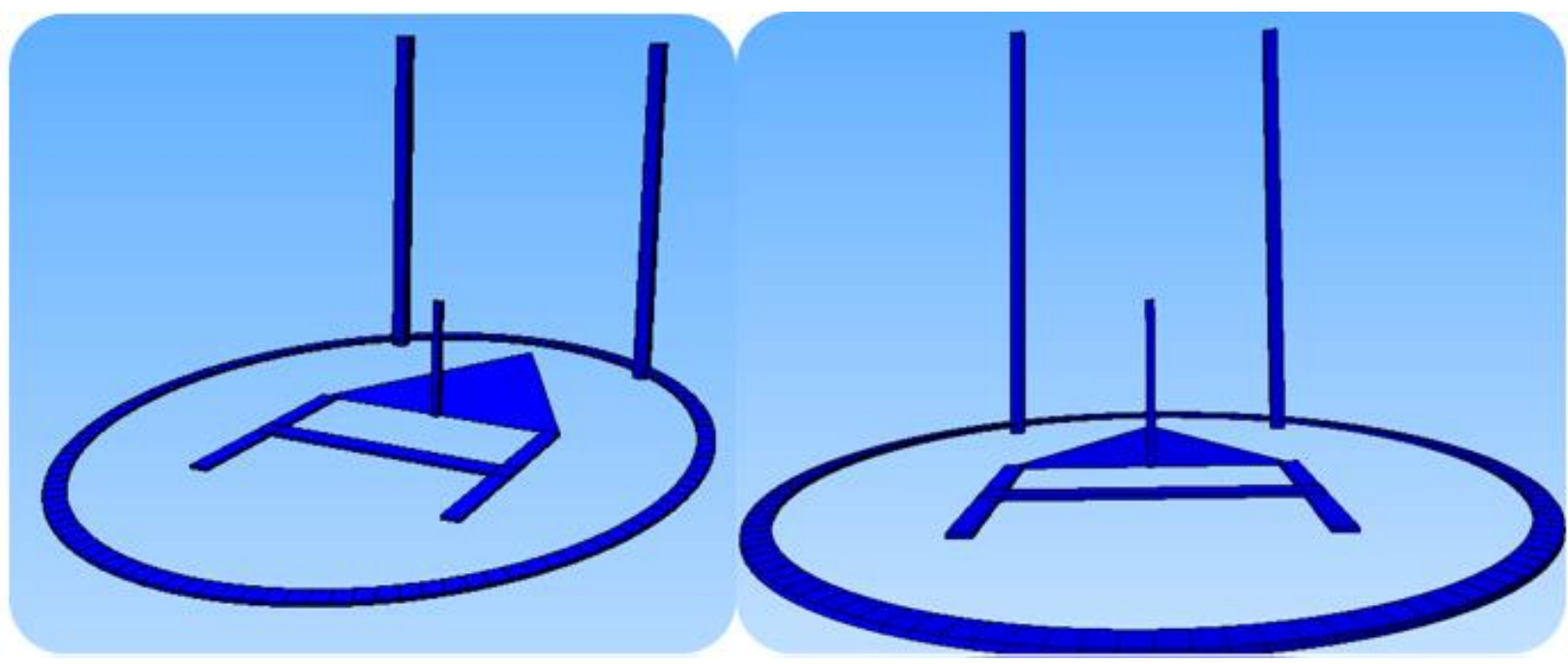

FIGURE 51: THE 3-D SYMBOLOGY DERIVED FROM THE TAXONOMIC AIRCREW ERROR FRAMEWORK.

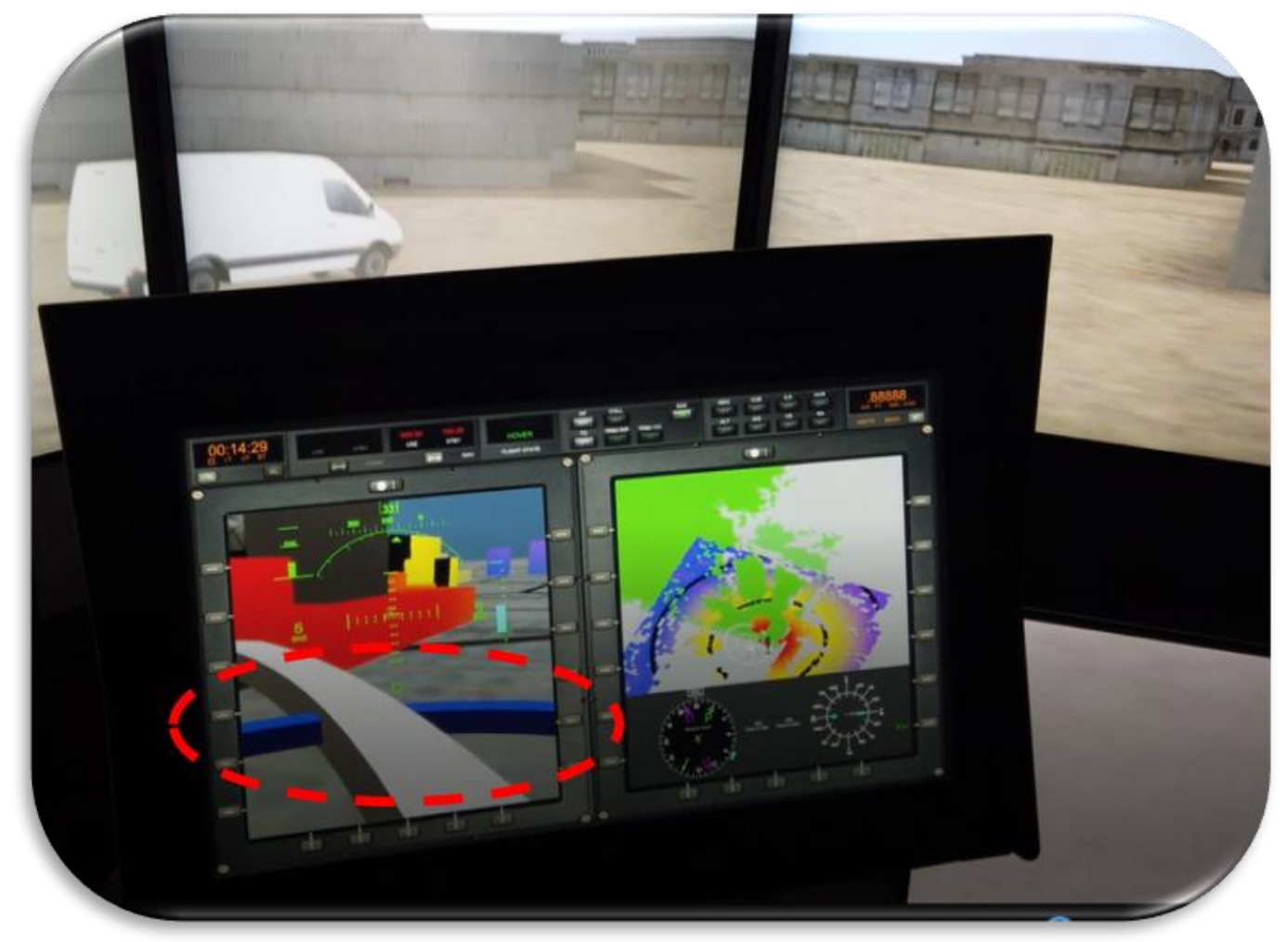

FIGURE 52: THE DVENS DISPLAY WITH THE FORWARD VIEW AND TOP DOWN VIEW MODE. IN THE FORWARD VIEW MODE, THE ROTOR DISK REPRESENTATION IS SHOW CIRCLED IN RED. THE RED SQUARES IN THE FORWARD DISPLAY CORRELATE TO THE VAN SHOWN IN THE OTW. 


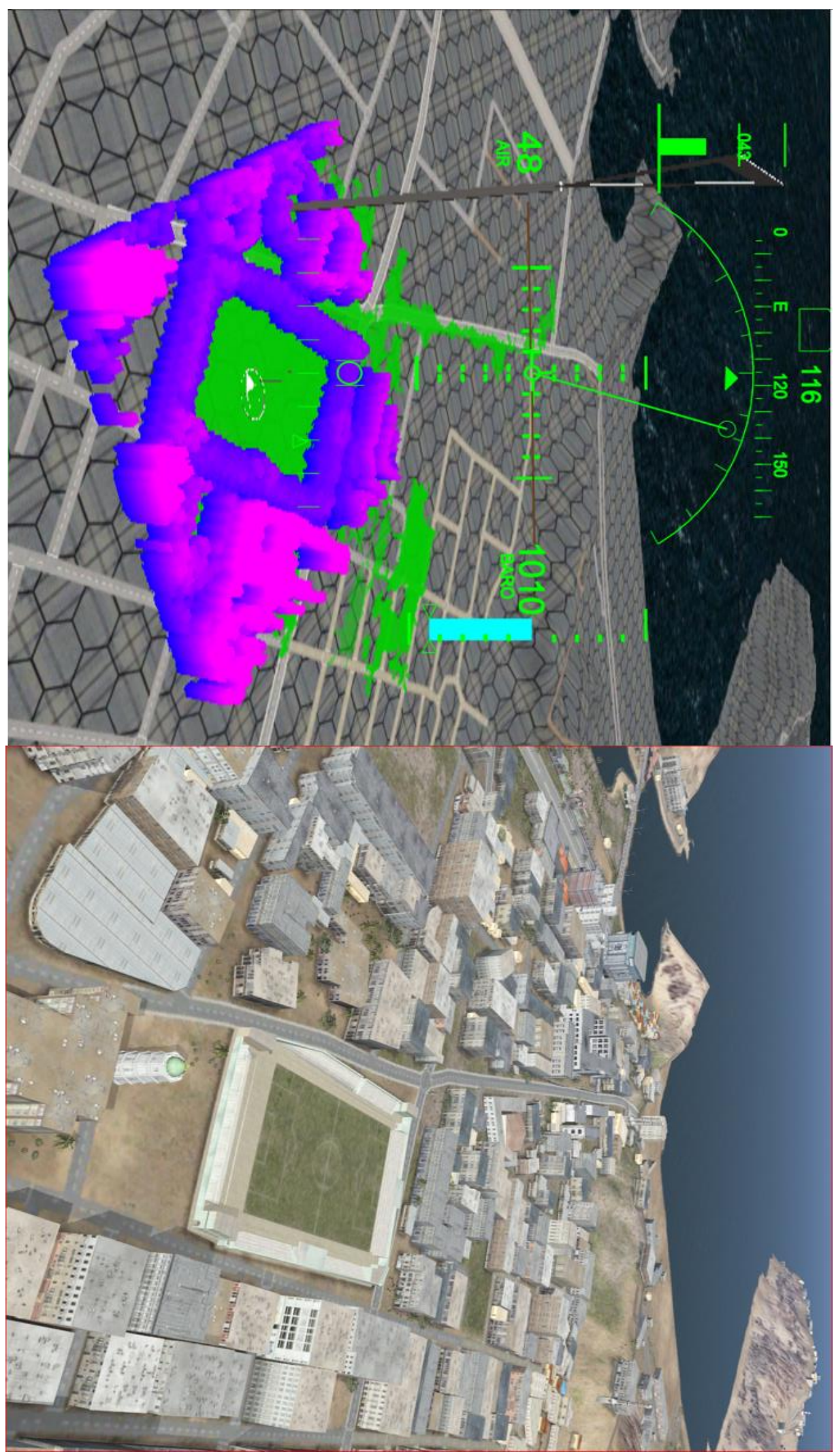

FIGURE 53: THE 3D AND 2D SYMBOLOGY WITH OBSTACLE INFORMATION SHOWN IN ONE WINDOW, THE RIGHT SIDE SHOWS THE OTW VIEW. 


\subsection{TERRAIN TEXTURE REPRESENTATION}

Chapter 2.5 provides a basis for the need to use textures to represent the terrain and augment the DEM/DTED data. The DEM is developed into CDB which contains minimally shaded models that highlight the terrain. Textures are used to perceive this terrain better. Numerous factors have been found to influence the perception of surface attitude and speed of the helicopter relative to the ground. A brief summary of the findings are highlighted below;

1. Doubly oriented two directional textures provided greater performance in judging attitude.

2. Textures carry information about distance along the principal direction

3. Symmetry seems to be an important character, with radial symmetry providing greater understanding.

4. The perspective projection was required for accurate surface orientation classification

5. Grids were found to be powerful enough to overcome motion parallax cues

6. In top-down plan view mode, contours were found to be far more effective.

7. Optimal performance is obtained with symmetric textures containing an orthogonal linear structure.
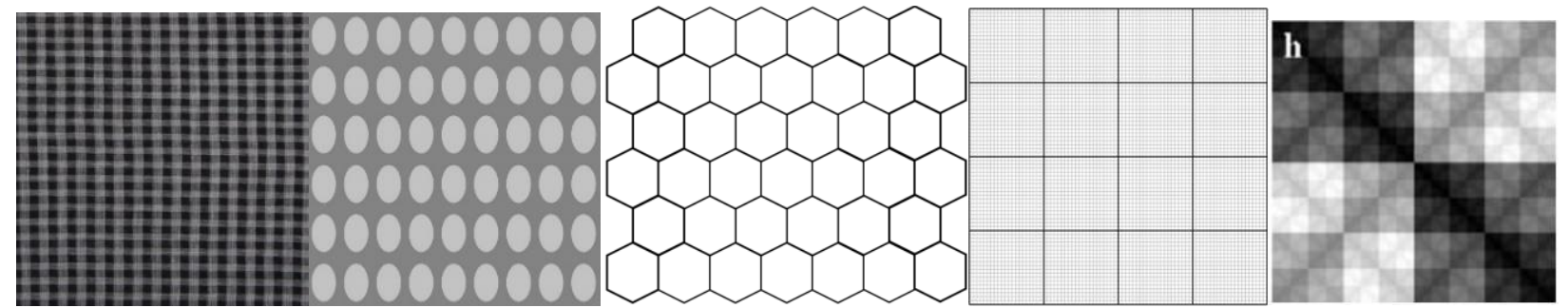

FIGURE 54: TERRAIN TEXTURE THAT WERE TESTED DURING FLIGHT TESTING.

The textures were tested in static and dynamic environments, static testing of the created textures. The patterns/textures above (Figure 54) were all the first set of developments from which the following observations were made.

1. Irregular patterns indeed provided very disturbing perception quality.

2. Regular patterns with use of highly prominent texels was damaging for perception.

3. Radial symmetry acted to provide a better perception for both small and large attitude changes.

4. Texel size played a crucial role, small texels were very beneficial in showing attitude over small changes. Larger texels were prominent in showing a larger surface attitude.

5. Texel shape and sharpness also made a significant difference in the perception of speed and attitude.

6. Major and Minor lines were highly beneficial in identifying attitude changes from high altitudes and low altitudes respectively. The use of major lines acts as contour lines, providing perception at larger viewing distance. 
7. When in low altitude hover, texel sizes played a crucial role, small texels were good at low altitude and large texels were good at higher altitudes.

8. Introduction of individual textures helped perception at low altitudes, that was otherwise less helpful with texels alone. (Microtextures).

9. Circular or radial texels provided a good representation of slope, however, were found too disturbing while over flat terrain.

TABLE 5: SUMMARY OF TEXTURE PATTERN TESTED WITH PROPERTIES AND ISSUES EXPERIENCED.

\begin{tabular}{ll}
\multicolumn{1}{c}{ Name/Figure } & \multicolumn{1}{c}{ Issues Faced } \\
Checkerboard (A) & $\begin{array}{l}\text { Fuzzy characteristics, bad in } \\
\text { forward motion flight } \\
\text { perception, good at long distance } \\
\text { attitude perception. }\end{array}$ \\
'H' Pattern (B) & $\begin{array}{l}\text { Too active as is to present terrain } \\
\text { attitude, good as microtextures. }\end{array}$ \\
Polka Dots (C) & Good slope perception, however \\
& very uncomfortable for viewing \\
in dynamic flight scenarios. & The radial shape helps slope and \\
Hexagonal Major, Minor and & attitude perception like polka \\
Grids Major, Minor (D) & dots, but lack of solid color is \\
more comfortable.
\end{tabular}

From the static testing of initial textures shown in Figure 55, they substantiated findings from Chapter 2.5. It was found from static testing that hexagonal radial pattern and the grid texture pattern were better in understanding surface attitude over other textures. The checkboard texture was good over larger viewing distances, however, confusing in low altitude flight, due to its 'fuzzy' characteristics. The polka-dots texture was tested to understand the effects of radial patterns in shape perception. The round texels provided right slope and attitude perception 'flowing' with the terrain and highlighting all the curvatures. However, it was uncomfortable to the eyes in both a static and dynamic environment. The findings of using are highlighted in Table 5. 


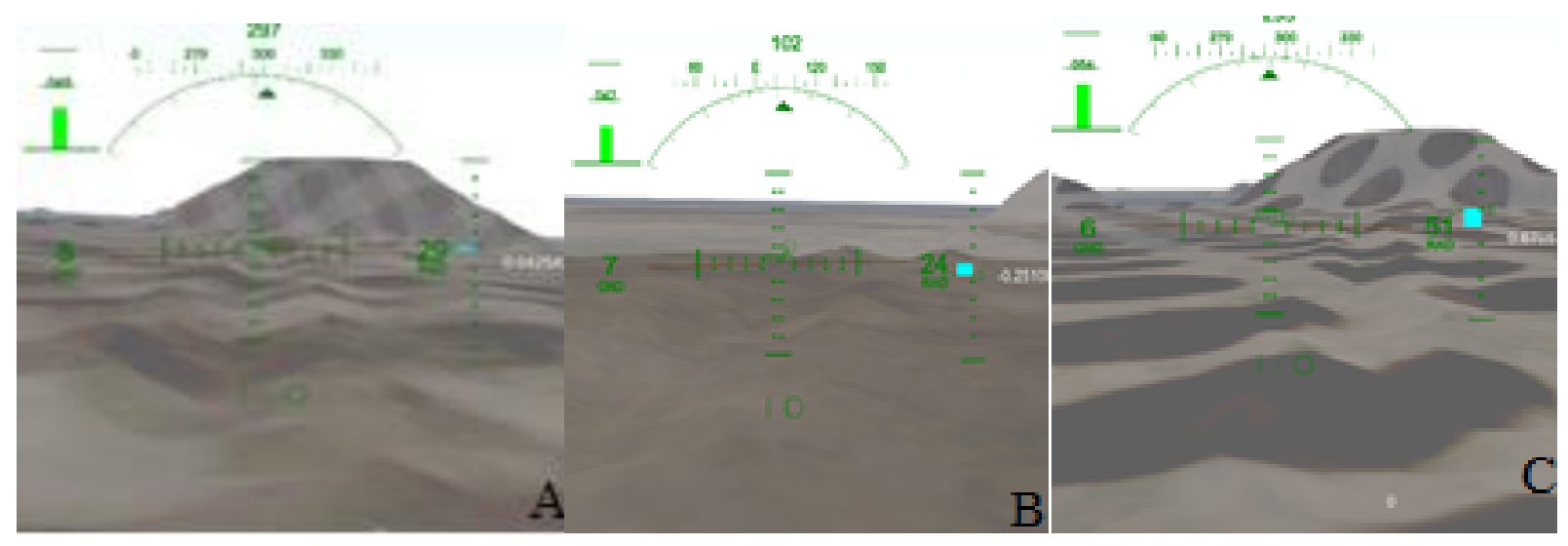

FIGURE 55: THREE EXAMPLE TEXTURES (PLAID PATTERN (A) TWO DIMENSIONAL GRIDS (B) AND POLKA DOTS (C)) WHICH UNDERWENT TESTING OVERLAID ON A DEM WHILE MAINTAINING A LOW HOVER.

As can be seen from Figures 55 (A, B, and C), different levels of perception of attitude, altitude, and slope can be attained with different texture types. The ditch is more clearly visible in Figure 55 (laid with texture A), as opposed to Figures 55 (laid with textures B and C respectively). Other terrain features are also highlighted with the use of terrain textures. It was found while hovering at low altitude the drift perception was reduced with the tried textures, the texels were too big for low hover, and small drifting characteristics were not easily perceived.

Upon initial testing, it was found that the hexagonal, checkerboard and grid pattern showed promising results with good perception in both larger altitudes $(>100 \mathrm{ft})$ and low-level flight (10-50 ft). The textures however did not provide the necessary perception while in low hover. When in low hover, the texels were too big to provide precise drift information. Referring to Chapter 2.3, pilots use microtextures to perceive drift and motion. Texture D-1 and D-2 from Figure 56 is underlined with a secondary micro-texture showing fine details. Thus, overlaying textures $\mathrm{D}$ from Table 5 over a grainy micro-texture yields a formidable tool for both high altitudes and low altitude flight (shown in Figure 57 and 58). This overlay of grid and hexagonal patterns with a 'mesh', enables distinction at very low and high altitudes.

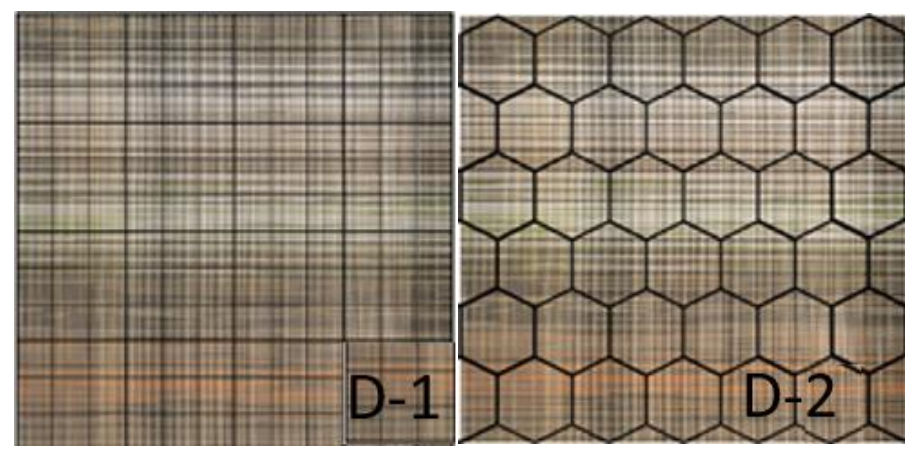

FIGURE 56: TWO OF THE FINAL TEXTURES SELECTED FROM EXTENSIVE FLIGHT TESTS, THE SANDMESH-BOX AND SANDMESH-HEX. 


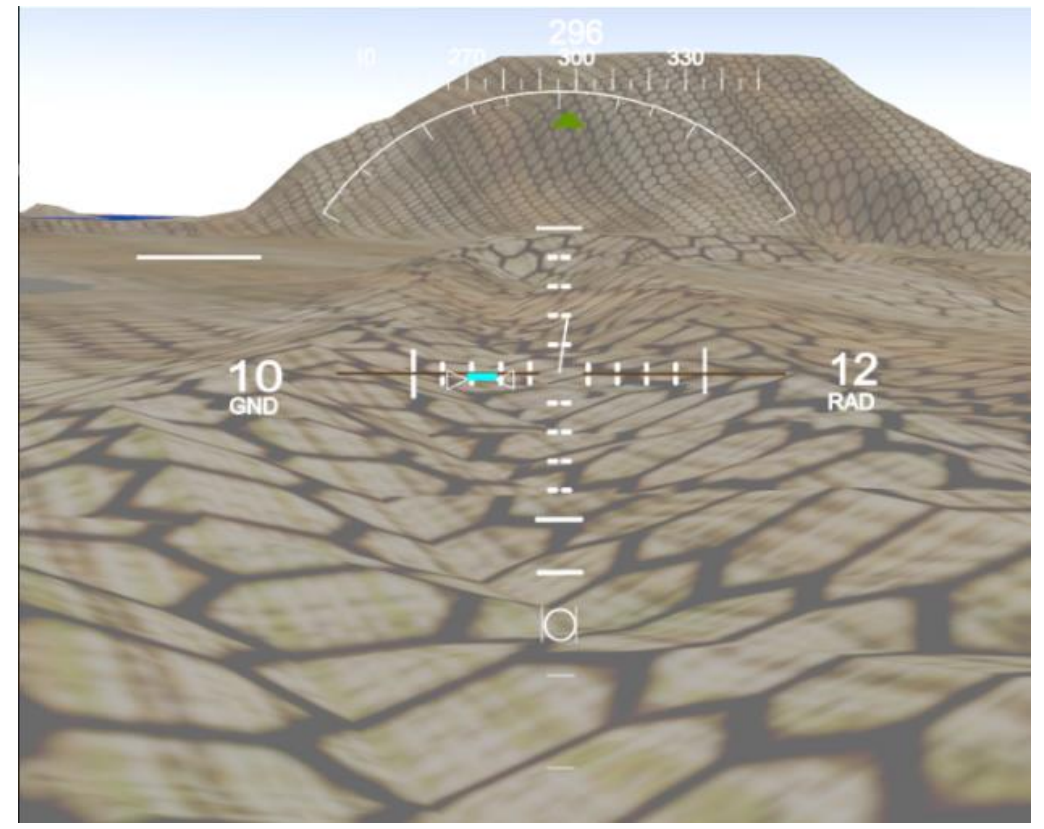

Figure 57: THE D-2 SANDMESH HEX TEXTURE OVERLAID ON TOP OF THE TERRAIN AT APPROX. 10 FT AND 10 KNOTS.
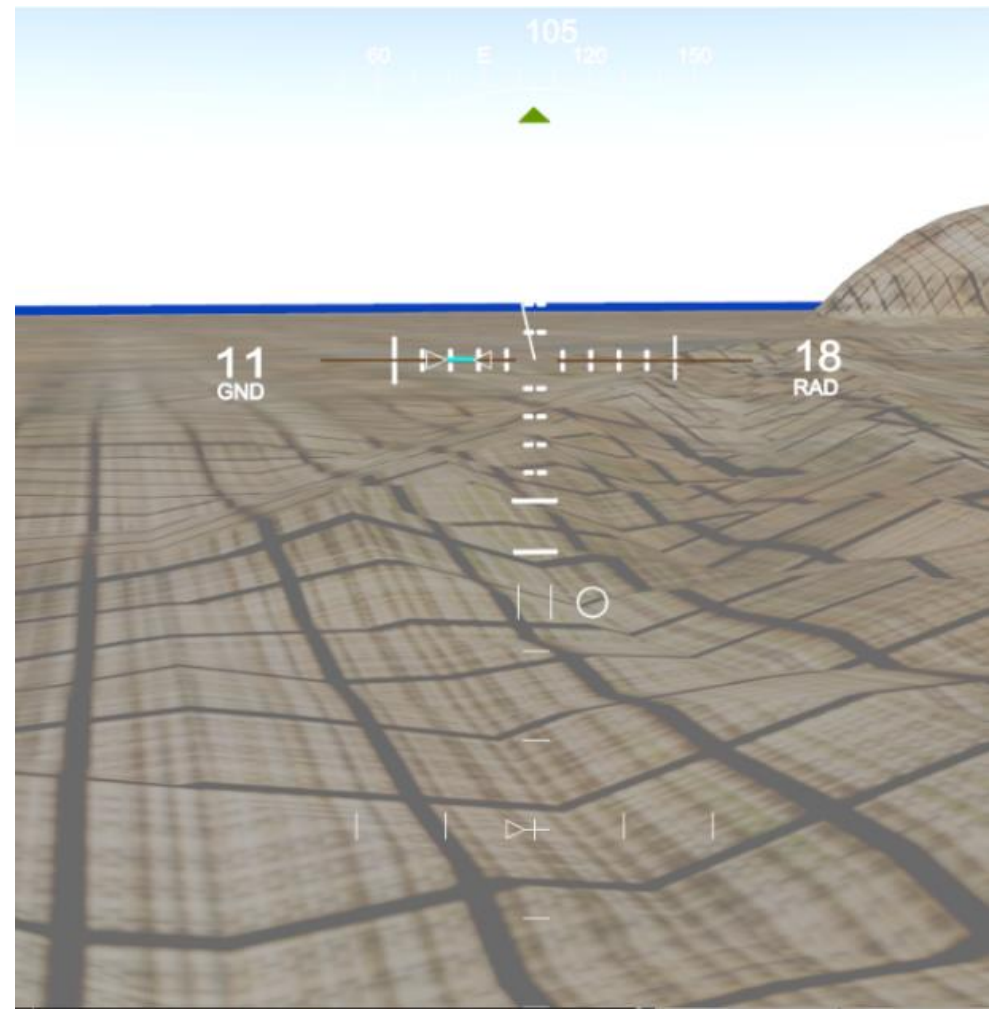

FigURE 58: THE D-1 SANDMESH BOX TEXTURE OVERLAID ON TOP OF THE TERRAIN AT APPROX.10 FT AND 10 KNOTS. 
Figures 57 and 58 shows the final chosen textures (Figure 56 D-1 and D-2) overlaid on top of the DEM/DTED data. Figure 57 exhibits the SandMesh HEX and Figure 58 exhibits the SandMesh Box textures patterns overlaid on the Yemen Terrain Database from CAE, Presagis.

The texture development is utilized for a forward-facing view mode. The SA requirements call for a secondary view mode, a planform perspective. Planform mode mainly acts to provide overall situational awareness around the helicopter and provide necessary cultural information along with elevation information, something like charts available to pilots in cockpits. Referring to Chapter 2.5, a banded contour line rainbow texture is found to be the most beneficial for relaying elevation information. However, the color palette leads to issues; red, green amber is used to signify different elevation levels. In the DVENS context, this color palette is used to represent the near and far-field of the LiDAR reach. Thus, a different color palette and contour system are used to represent elevation information. A simpler, greyscale-based color palette was chosen with black contour lines providing the necessary elevation understanding. The lighter (white) means higher elevation while darker (gray/black) means lower elevation. Individual contour lines are spaced 100-meter each.
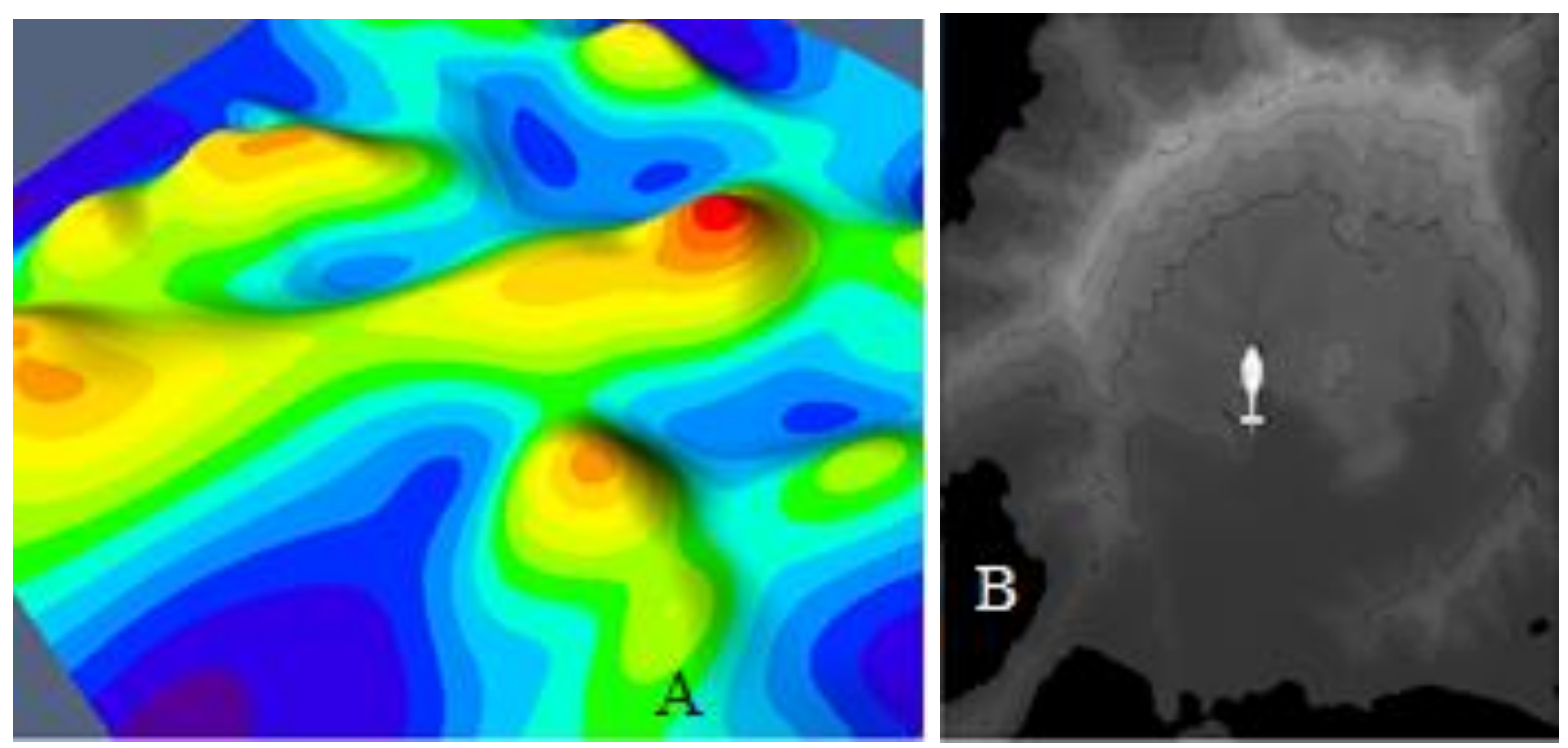

FIGURE 59: EXAMPLE OF A BANDED RAINBOW (A) TYPE CONTOUR LINE ELEVATION DISPLAY AND THE CHOSEN DISPLAY, WHICH FOLLOWS A CONTOUR BANDED PRINCIPLE IN A GREYSCALE PALETTE (B).

The overall testing and implementation showed that the chosen two textures in Figures 56 (D-1 and D-2) are for the forward view modes (seen in Figure 57 and 58), while Figure 59 (A and B) highlights the texture chosen for the planform 'top-down' view mode. The choice of both these textures underlines the requirements to show what the pilot needs. 


\subsection{FILTERING DATA SOURCES}

A filter is an electric circuit capable of communicating, changing, or amplifying values from a source (sensor) to a display between origin and destination points. Filters are essential to process a signal, where the origin signals are modified or attenuated to isolate necessary signals and frequencies [72]. Signal processing and attenuation are essential to environments with high vibratory and noise infested operations like those experienced in helicopters. There is considerable signal noise due to rotors in a helicopter, and it is imperative to filter the noise to read and provide accurate information that is presentable. The information presented must be damped by limits and not too sensitive, as it will lead to overcorrections and pressure on the pilot.

There are four major types of filters; low pass filters, high pass filters, bandpass filters, and notch filters. Low pass filters are a circuit that allows low-frequency signals and attenuates high-frequency signals. High pass filter functions opposite to a low pass filter by allowing high frequencies to pass and attenuates low frequencies. A Bandpass filter is used for particular needs to be met, where bounds are utilized to make use of signals of a specific spread. A bandpass filter is utilized by a combination of properties of low pass and high pass filters into a single filter in series. A notch or band-stop filter is utilized to use signal frequencies in a specified range. It is also composed of the low pass and high pass filters connected in parallel.

As all helicopter operation require attenuation of signals at a certain point to filter the noise generated by the main rotors and other peripherals, filtering techniques like low-pass, high-pass, bandpass, and notch are used to pick specific or ranges signals. In helicopters such as the Bell 412, filters are used in the electrical systems to process incoming signals. The exact nature of filter system integrated into the Bell 412 helicopter system is unknown, however, with the collaboration of NRC FRL and utilization of their BELL 412/BELL 205 helicopter data system, it was retrieved the transmitting or operating frequency is $133.3 \mathrm{~Hz}$ [73]. The data system uses low pass second order Butterworth filtering to attenuate high signal frequencies, to allow signals of specified ranges to be passed. The Presagis HeliSIM operates at $100 \mathrm{~Hz}$; however, this operating frequency can be changed to any value.

Digital filters allow signal filtering in the time domain. Digital filtering performs mathematical operations on a sampled, discrete-time signal to reduce or enhance certain aspects of that signal. A Butterworth filter is a signal processing filter designed to have as flat frequency response as possible in the passband. It is also referred to as a maximally flat magnitude filter [72]. The Butterworth design is one of the best known and easy to implement filters, with a smooth roll-off and monotonic.

A generalized design principle can be utilized to produce the transfer function necessary to describe the filters which are utilized in the processing, attenuation of signals produced. The generalized set of equations 
can be formulated for the design of second-order low pass filters. As with any other filter design procedure, the designed characteristics of the filter are required.

$$
\begin{gathered}
f_{c} \text { or } \omega_{c} \text { : Cutoff frequency } \\
f_{s} \text { or } \omega_{s} \text { :Sampling frequency } \\
ב: Q \text { factor or Damping ratio } \\
\text { G:Boost or cut dB gain value at } f_{c} \text { (Peaking and shelving filters) }
\end{gathered}
$$

To create a $2^{\text {nd }}$ order low-pass filter is described by the transfer function in Equation 3.1.

$$
H(s)=\frac{\omega_{n}^{2}}{S^{2}+2 \beth \omega_{n} S+\omega_{n}^{2}} \ldots E q 3.1
$$

$\omega_{n}$ is the natural frequency and $S$ is a mathematical frequency domain and is transformed from the time domain to the frequency domain via a Laplace transform.

The Cutoff frequency is given by equation 3.2 and is described to be the frequency at which output to input ratio has a magnitude of 0.707 [72].

$$
\begin{gathered}
\omega_{c}=2 \pi\left(\frac{f_{c}}{f_{s}}\right) \ldots E q 3.2 \\
\omega_{f}=2 \pi\left(f_{s}\right) \ldots E q 3.3 \\
\text { Damping ratio }(\beth)=\frac{1}{2 Q} \ldots E q 3.4
\end{gathered}
$$

Equation 3.1 describes the transfer function utilized for filtering the data sources in the simulation system. For certain data sources, like the accelerations in lateral, longitudinal and vertical directions a notch filter is utilized. The notch filter is described via the transfer equation 3.5.

$$
H(s)=\frac{S^{2}+\omega_{n}^{2}}{S^{2}+2 \beth \omega_{n} S+\omega_{n}^{2}} \ldots E q 3.5
$$

The Presagis helicopter simulator package HELI SIM has the capability of defining its user-defined modules and outputs. This feature allows the researcher to define custom modules with the addition of transfer functions that describe the signal processing input. Such transfer functions are implemented in the program to take simulation data and apply custom filters to attenuate the source values to reduce vibratory effects or filter towards real world conditions. 


\subsection{NAVIGATION VIA WAYPOINTS AND LANDING ASSIST}

The ILS system is an essential and useful navigation system that helps pilot perform precise landings. In the context of the study, the ILS system was replicated on a simulation basis, creating a pseudo "runway". When the LiDAR identified a zone as being safe to land, and the pilot selects it, a similar architecture to ILS is implemented. When a zone is selected, a proxy ILS system is placed at the zone within the frequencies mentioned navigating and representing to the pilot the elevation and bearing deviations into land at the selected zone. This setup configuration led to some unforeseen issues in the development and initial flight tests, listed below;

- The system reacted erratically as the helicopter got closer to the LZ, the needle pointer moved too fast for the pilot to deduce information. When the range was too small and the deviation indication too sensitive, the Localizer presented limited positive capability. The pilot had to select, fly afar again, turn around to land with the use of ILS. Due to the wide casts angle of the specified frequency (18 degrees), as the helicopter got close to the landing zone, the CDI indicator seemed to be extremely sensitive, often confusing the pilot with erroneous data and representations as to how to maneuver.

- A high-fidelity simulator represents an accurate flight model along with functions, capabilities, and realism to the actual scenarios and environments. For ILS, the placement of virtual beacons is not feasible nor realistic in-flight scenarios i.e., not how an actual helicopter or landing system would function. The idea of the project is to prove methods that would help solve present a complete solution to the DVE issue. Thus, an alternative using Area Navigation (RNAV) is utilized in cohesion with CDI representation to achieve the solution.

With the presence of three issues that question the application onto a real helicopter, a different RNAV approach has been taken. This method does not require the use of external placements of beacons and frequency transponders in aircraft. The RNAV system works by navigating between two points on earth, with the inputs being the helicopter location and waypoints or destination's latitude, longitude and altitude. The implementation is performed using the Haversine method of navigation between two points.

Calculation of properties between two points of the earth can be conducted neglecting ellipsoidal effects, based on an assumption the earth is a sphere through the Haversine formulas, which are accurate up to $0.3 \%$ [74]. The Haversine methodology is used to calculate properties about navigation between two points on the surface of the earth while not treating it as a plane but rather a plane of a degree of curvature. 


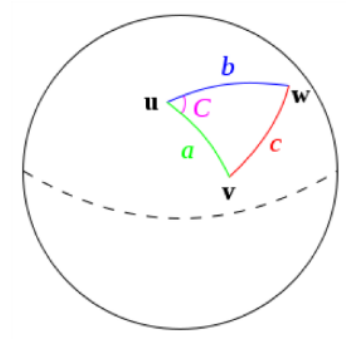

FIGURE 60: SPHERICAL TRIANGLE SOLVED BY THE LAW OF HAVERSINES [75].

Haversine algorithm establishes the direct distance between points that can be stretched in a triangular form such that the values of $a, b$ and $c$ can be calculated[74].

$$
a=\sin ^{2}\left(\frac{\Delta \varphi}{2}\right)+\cos \left(\varphi_{1}\right) \cos \left(\varphi_{2}\right) \times \sin ^{2}\left(\frac{\Delta \varphi}{2}\right) \ldots E q 4.1
$$

Angular distance in Radians : $c=2 \times \operatorname{atan} 2(\sqrt{a}, \sqrt{(1-a)}) \ldots E q 4.2$

$$
\text { distance: } d=R \times c \ldots E q 4.3
$$

$\varphi_{1,2}:$ Latitude of points 1 and 2

$$
\gamma_{2} \text { : Longitude of points } 1 \text { and } 2
$$

\section{$R:$ radius of the sphere.}

To compute the bearing that needs to be maintained in order to navigate to a location, the haversine equation can be used [74]:

$$
\theta=\operatorname{atan} 2\left(\sin \Delta \gamma \times \cos \varphi_{2}, \cos \varphi_{1} \times \sin \varphi_{2}-\sin \varphi_{1} \times \cos \varphi_{2} \times \cos \Delta \gamma\right) \ldots E q 4.4
$$

A midpoint between the $\mathrm{LZ}$ and helicopter is retrieved by;

$$
\begin{gathered}
\mathrm{B}_{\mathrm{x}}=\cos \varphi_{2} \times \cos \Delta \gamma \ldots \mathrm{Eq} 4.5 \\
\mathrm{~B}_{\mathrm{y}}=\cos \varphi_{2} \times \sin \Delta \gamma \ldots \mathrm{Eq} 4.6 \\
\varphi_{\mathrm{m}}=\operatorname{atan} 2\left(\sin \varphi_{1}+\sin \varphi_{2}, \sqrt{\left(\cos \varphi_{1}+\mathrm{B}_{\mathrm{x}}\right)^{2}+\mathrm{B}_{\mathrm{y}}^{2}}\right) \ldots E q 4.7 \\
\gamma_{\mathrm{m}}=\gamma_{1}+\operatorname{atan} 2\left(\mathrm{~B}_{\mathrm{y}}, \cos \left(\varphi_{1}\right)+\mathrm{B}_{\mathrm{x}}\right) \ldots E q 4.8
\end{gathered}
$$

In the DVENS application of navigation, the helicopter is the initial location, and the pilot chooses the SLZ as the destination. When a pilot selects from the helicopter HDD, the Haversine method is implemented to provide the respective bearing, elevation to reach the waypoint. In the context of real helicopter operations, 
a pilot would enter the respective latitude, longitude and altitude and navigation provided to a waypoint/landing site through trajectory. The difference in the current helicopter heading and the heading prescribed by the haversine results is the bearing deviation to a waypoint or SLZ. It is represented via the $\mathrm{CDI}$ or localizer, a 2D representation of bearing deviation. Similarly, the simple difference in the elevation of the helicopter and the elevation at which the waypoint or SLZ are located drives the Glideslope representation vertically.

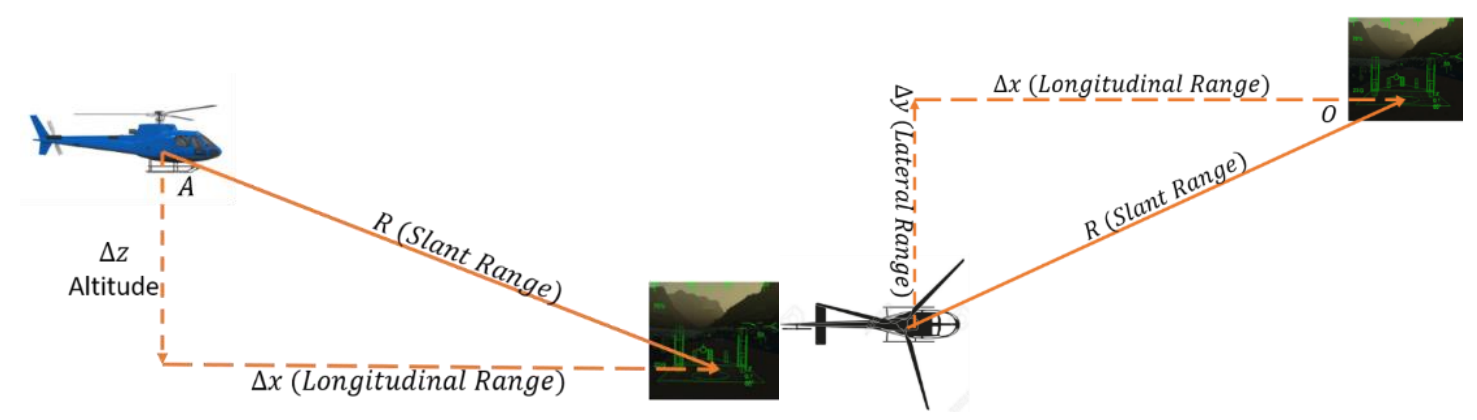

FIGURE 61: REPRESENTATION OF RANGE AND DEVIATION CALCULATION.

\section{SCALING OF THE LZ MARKER}

The LZ marker is displayed when the pilot would select a landing point and is displayed according to scale based on the range between the helicopter and chosen landing point. The LZ marker is displayed at the top edge of the screen signifying maximal distance and at the center when the helicopter is in hover/landed at the selected point. A scaling calculation is performed to accommodate and provide the pilot with a continuous understanding of the acceleration required to maintain accurate descent characteristics.

$$
Z_{i}=\frac{x_{i}-\min (x)}{\max (x)-\min (x)} \ldots E q 4.9[76]
$$

The maximum selectable distance is $\max (x)$ and the minimal selectable distance is $\min (x)$, and the current distance/range from the landing point is denoted by $x_{i}$. This allows the selected LZ or landing point to be represented as a graphic on the display. This capability allows the pilots to place the acceleration or velocity vector cue in the $\mathrm{LZ}$ marker to accelerate/deaccelerate the helicopter to the landing point. Scaling can be performed to set the target speeds to provide deceleration cueing or for waypoint speed cueing. The target speed is calculated via Eq 4.10

$$
V_{T}=D_{C}\left(\frac{V_{I}}{D_{I}}\right) \ldots E q 4.10[77]
$$

$V_{I}$ is the current speed, $D_{I}$ is the distance to the landing site when speed guidance has started, $D_{C}$ is the current distance to landing site. $V_{T}$ is the current target speed established. 


\subsection{FLIGHT PARATMETERS AND TRANSISIONS}

The developed displays are suitable for multiple regimes of helicopter flight, as a helicopter can hover and cruise the respective symbology must be displayed at the right times. The hover symbology implemented is only useful for slower and low altitude flight, while cruise symbology is more suited for faster flight scenarios. When switching between the two different flight display modes, the system is susceptible to switching between the displays rapidly and flicker when the pilots are operating close to transition. Flicker is eliminated via the use of hysteresis. For the Bell 412, the transition condition for hover to cruise symbology and viseVersa at 60 knots [78]. Figure 62 describes via flow chart the display conditions on how to switch between the two symbology.

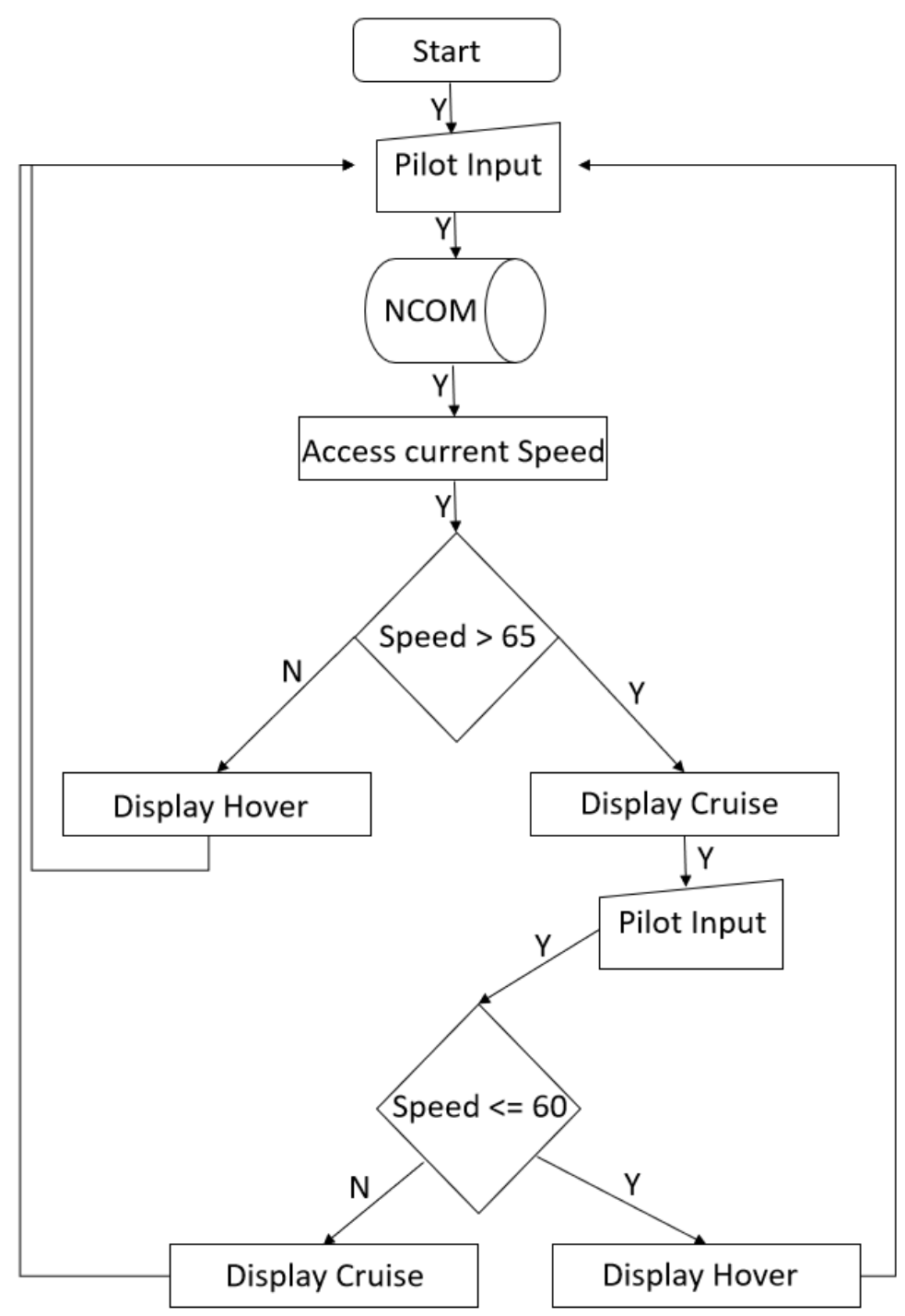

FIGURE 62: ALGORITHM FLOWCHART FOR HOVER/CRUISE SYMBOLOGY TRANSITION [78]. 
Similarly, at different stages of flight, transitions must occur that present to the pilot different flight data. Above a dictated altitude, the reference altitude and speed also change; the changes are described via flowcharts in Figures 62 and 63. The torque representation of the helicopter is represented via a bar graph which changes color, based on threshold values dictated by helicopter operational capabilities.

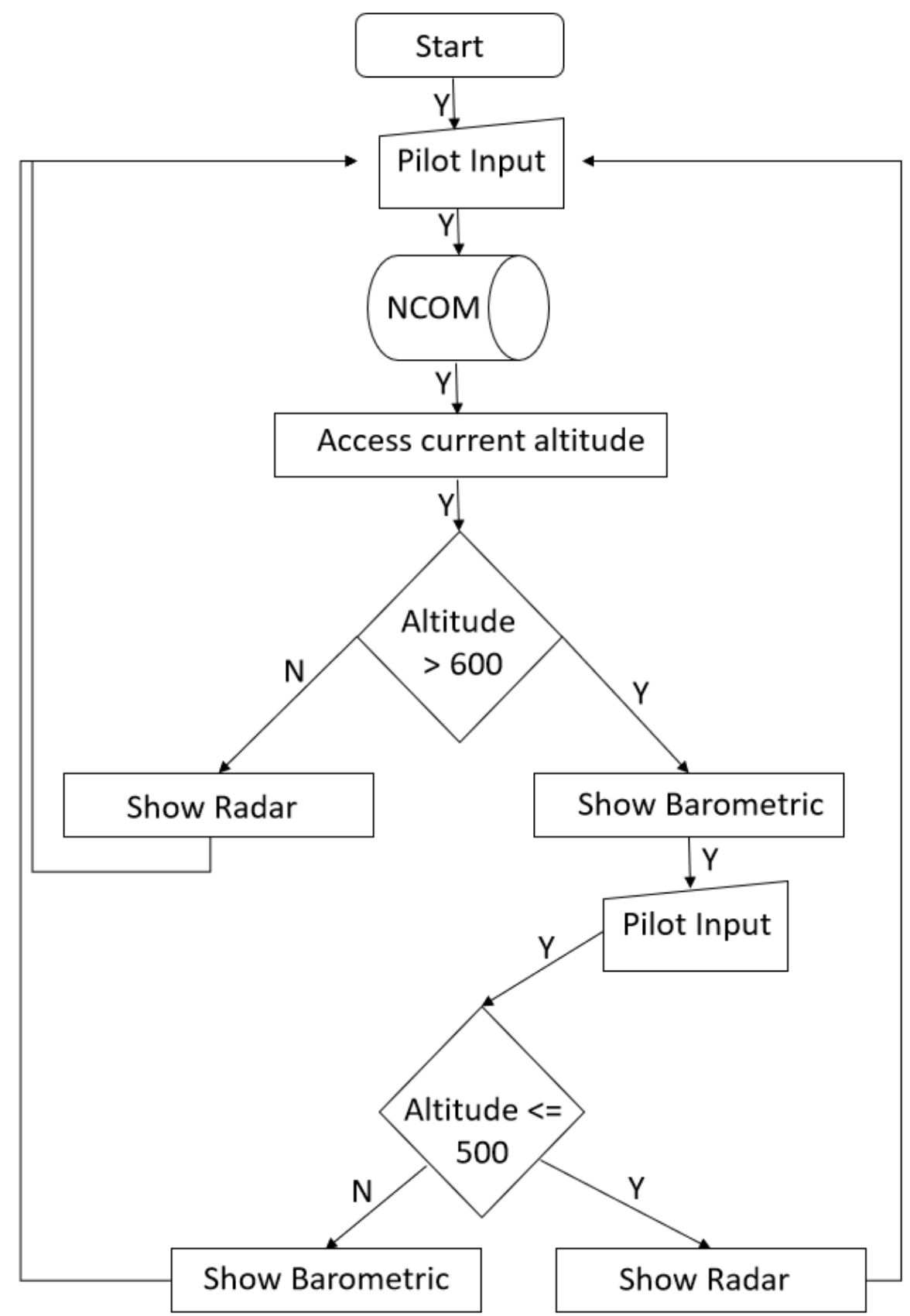

FIGURE 63: ALGORITHM FLOWCHART FOR ALTITUDE SOURCE TRANSITION BETWEEN BAROMETRIC AND RADAR ALTITUDE [78]. 
A semi-autonomous approach is used to help the pilots rotate the 3D symbology. In a previous design iteration, pilots are given full authority to choose the orientation of the 3D conformal symbology through a rotary knob. Pilots reported excessive physical and mental workload due to last-second changes to the orientation to align with the ground track. The pilots had to operate the cyclic, collective, pedals while trying to change orientation to match that of the helicopter. The combined task of landing and orientation selection was extremely difficult for the pilots. Thus, more automated approach was selected. Since the pilots were always trying to match the ground track of the aircraft or landing into the wind direction, a lock/unlock feature was established. When toggled to 'Lock' the rotation stops following the aircraft ground track and assumes the orientation of the wind. When toggled to 'unlock' the symbology rotates to orient with the aircraft ground track while outside a 500-meter/1604-feet range. Thus, a pilot performing a hover or roll-in landing can set the orientation into the wind or maintain the ground track orientation on the 3D symbology. The semi-auto approach allowed the pilot to have control and over the orientation. Figure 64 highlights the algorithm used to for the classification of rotation/no-rotation of the symbology. Figure 65 shows the interactive button (yellow helicopter representation) which is used to toggle between the heading source.

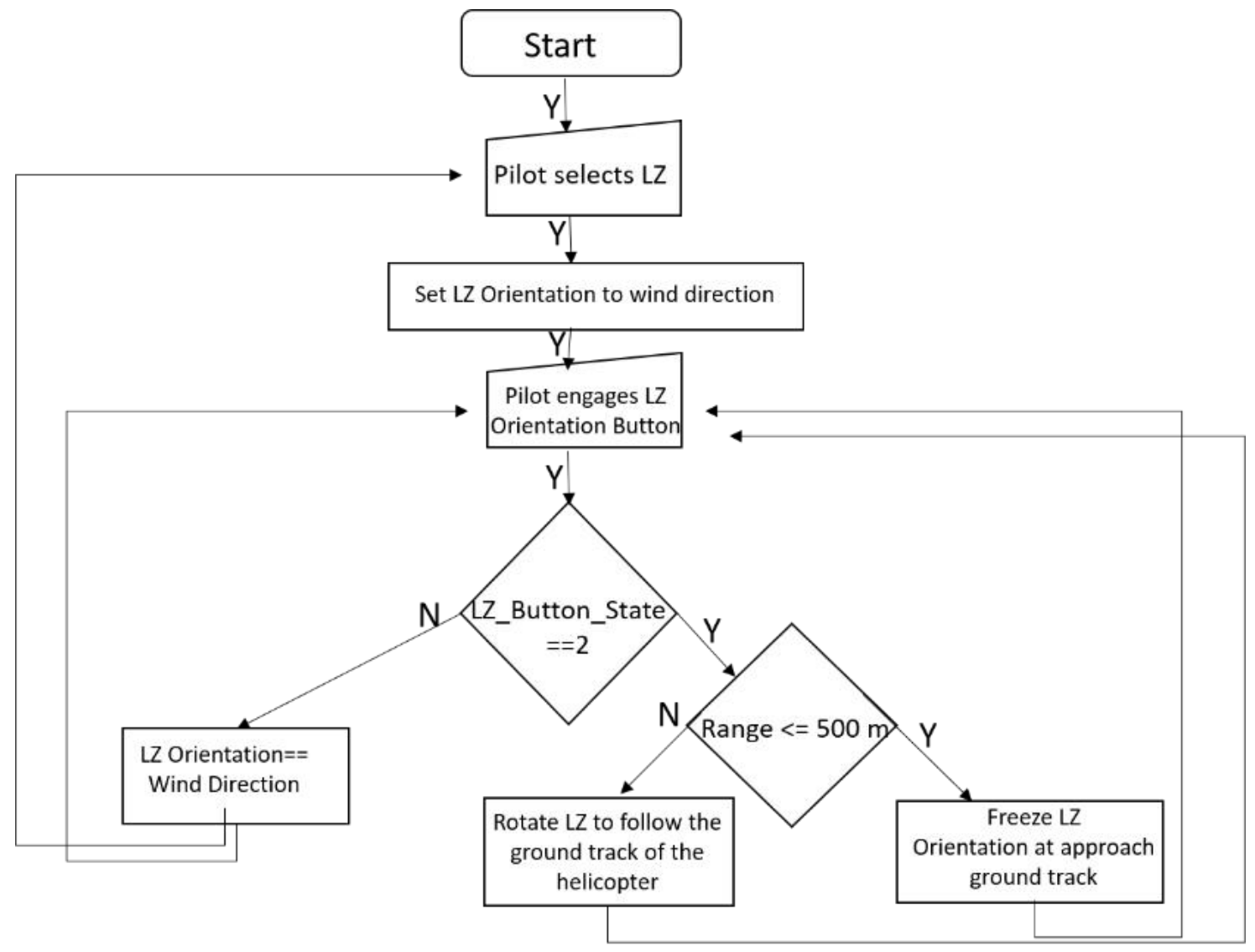

FIGURE 64: ALGORITHM LOGIC FOR ROTATION OF THE LANDING ZONE SYMBOLOGY [78]. 


\subsection{AUXILLIARY SYMBOLOGY}

Auxiliary symbology enhances overall understanding of the scenario, it is not a part of the primary symbology, it is a tertiary source of information which provides more task and spatial understanding. Task awareness is a critical component of the SA cluster; the pilot must beware of the task at hand with accurate objective and time to objective information. To provide the pilot with information on the task, time to objective/waypoint, and time to mission/destination is provided. Thus, the pilot would have a task and objective awareness based on time. As part of reducing the workload and providing the pilot with capabilities in DVE, the 3D symbology rotation was implemented. A section of the 2D orthogonal display is the Auxiliary symbology (shown in Figure 65), which displays the present ground track of the helicopter and the locked/unlocked state of the 3D symbology rotation. Wind direction is a critical factor which affects the hover, take-off, and climb characteristics of a helicopter. Relative wind causes a translational lift in the rotor disk, with changing intensities and directions of winds, the helicopter is susceptible to oscillations and tailwinds, which require tail corrections to maintain heading [26]. When facing into the wind (headwinds), the translational lift increases, and less power is required to take off and hover.

Thus, the pilot must obtain an understanding of the wind conditions; they retrieve wind information from the environment, such as blowing trees, grass, or other markers. A wind direction and speed indicator were introduced to supplement the pilot's understanding of the environmental conditions and spatial awareness.

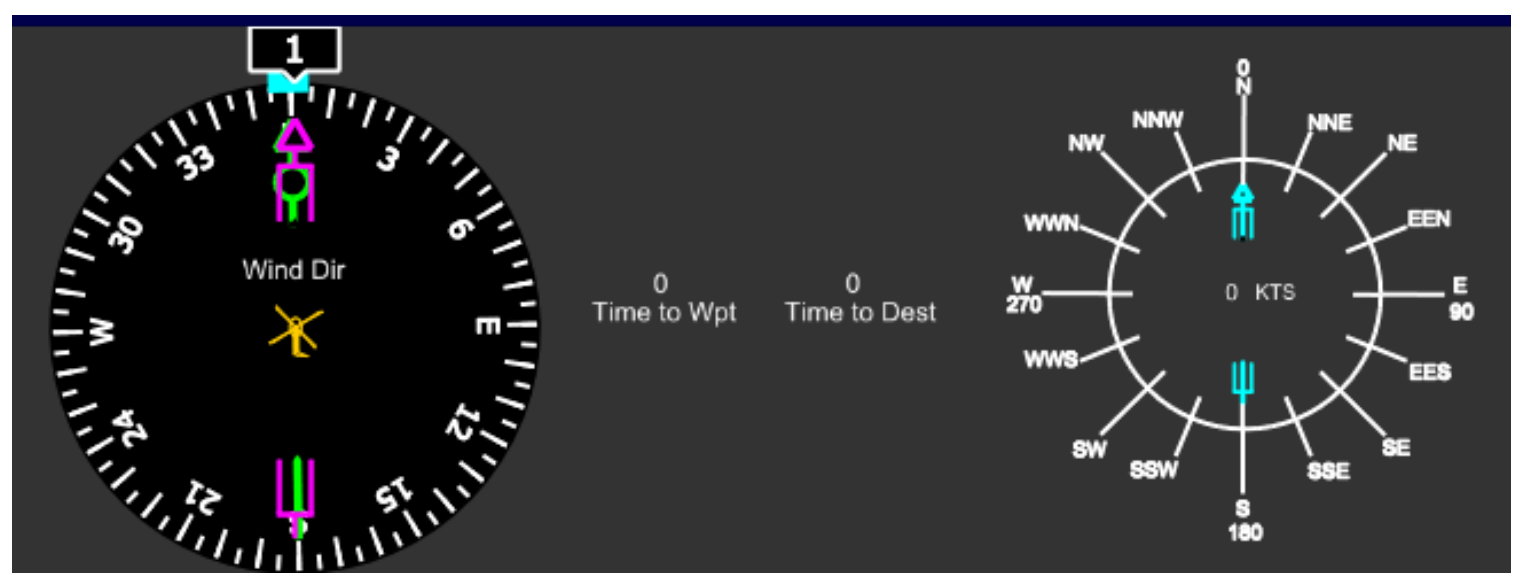

Figure 65: AUXILIARY SyMbOLOGY DEVELOPED USING PRESAGIS, CAE VAPS XT AT RYERSON UNIVERSITY. 


\subsection{FINAL SYMBOLOGY}

Based on the original design decisions undertaken, valuable initial feedback by the pilots was provided through initial flight tests that provided qualitative information as to changes that should be made to improve pilot perception. The pointers provided by the pilots suggested the removal and or repositioning of symbology to accommodate visual attention in time-critical situations. Prior to the final flight test, various versions of the symbology have been developed and tested in-flight demonstrations. No metrics or data were collected. However, suggestions as to how the symbology can be improved have been collected. The changes are based on pilot input of how the symbology components helped, and why it was or was not needed. There was no flight test data on the particular components that were removed, but the suggestions and comments from the test pilots explain their lack of importance in the scenario, which goes on to highlight important design considerations for future works.

1. The pilots suggested the removal of the altitude drift indication bar. They reasoned that the vertical velocity indicator, radar altitude reading, LZ marker, and Glide Slope (Altitude Deviation) as part of ILS provide a significant number of cues. An additional 'pillar' was however added at the center of the $\mathrm{H}$, so the pilots have better alignment when in low hover or low approach. The pilot would use the third pillar and position themselves about the pillar before touching down.

2. $3 \mathrm{D}$ waypoint representations are used to show the pilots where they need to fly, this is in addition to the ILS method for landing, ILS would be specifically used for landing while 3D waypoint is used to navigate to a staging/landing area or zone.

3. New symbology was added, which the pilot would not need 'at all times' information that would provide global objective information or situationally required information. During landing, pilots would need information about the wind conditions, waypoint information on the way to the objective location, etc. Thus, the following information was added as symbology on the other view mode.

a. Wind direction and wind speed indicator

b. Time to next waypoint information, absolute information for next waypoint.

c. Time to landing zone based on current aircraft orientation and position.

The suggested changes were implemented and have been the result of the final form of the symbology for both 3D conformal and 2D orthographic. The final flight tests which were conducted and from which the results were obtained are drawn from this form of symbology. Figure 66 shows the complete 2D, 3D and Auxiliary symbology that the pilot would use in addition with obstacle representation. 


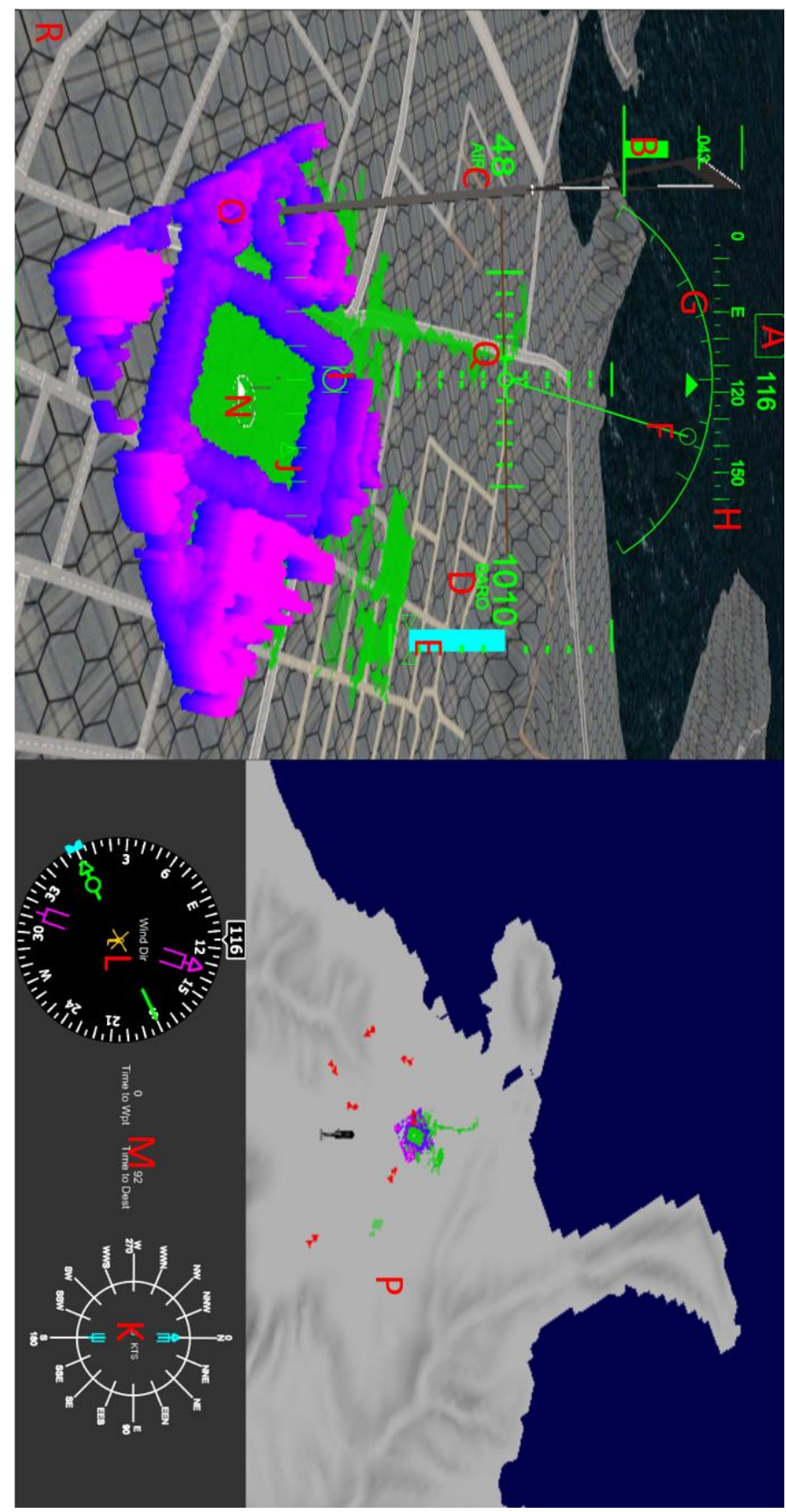

FIGURE 66: THE COMPLETE DVENS SYMBOLOGY WITH OBSTACLE REPRESENTATION, FOR DESCRIPTION OF THE LABELLED COMPONENTS PLEASE REFER TO TABLE 4 IN CHAPTER 4.2.

The labelling in figure 66 of individual components of the symbology are referenced in Table 4 for the 2D symbology. For the those not found in Table 2, they are listed here:

1. $\mathrm{O}$ is the Obstacle representation

2. $\mathrm{R}$ is the terrain texture representation

3. $\mathrm{N}$ is the 3D symbology

4. $\mathrm{P}$ is the top-down/planform view mode

5. $M$ is the waypoint time information
6. $\mathrm{K}$ is the wind direction and speed indicator

7. $L$ is the interactive touch $3 D$ symbology rotation button. 


\section{DEVELOPMENT AND EVALUATION OF CUEING SYMBOLOGY FOR ROTORCRAFT OPERATIONS IN DEGRADED VISUAL ENVIRONMENT (DVE)}

\section{Vamshi Chittaluri}

Master of Applied Science, Aerospace Engineering, Ryerson University, Toronto (2019)

\section{CHAPTER 5.0 EVALUATION AND RESULTS}

\subsection{FLIGHT AND TEST PLAN}

The testing of the developments of the DVENS project from a display standpoint, the development is composed of three major components. These are the 3D symbology, 2D symbology, and Terrain Textures. The goal of the test is to test the overall importance of the improvement the system brings about in DVE operations. Thus, the testing was divided into three respective components. Test subjects were asked to fly missions using a generic standard helicopter PFD (shown in Figure 67), 3D symbology and 2D symbology. The Terrain textures were individually tested as they required a high precision flight test. The following test cases were used to test the terrain textures, 3D symbology, and 2D symbology.

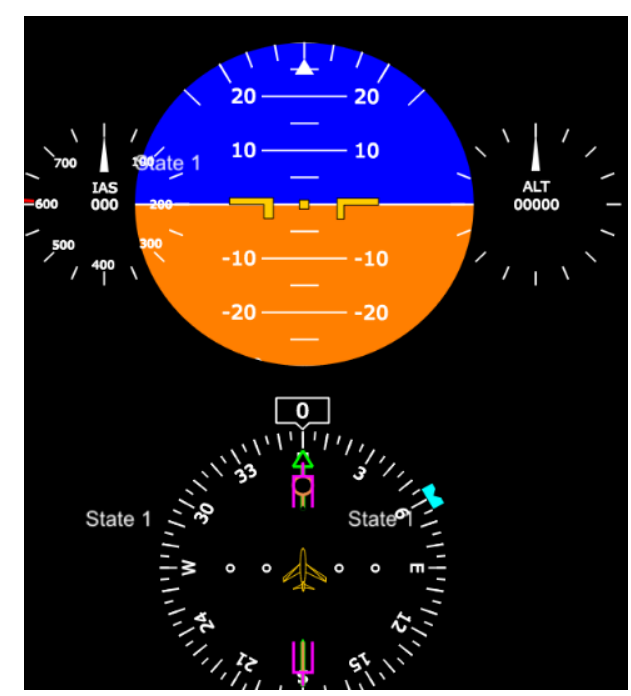

FIGURE 67: THE PFD SYMBOLOGY USED FOR THE FLIGHT TEST. 


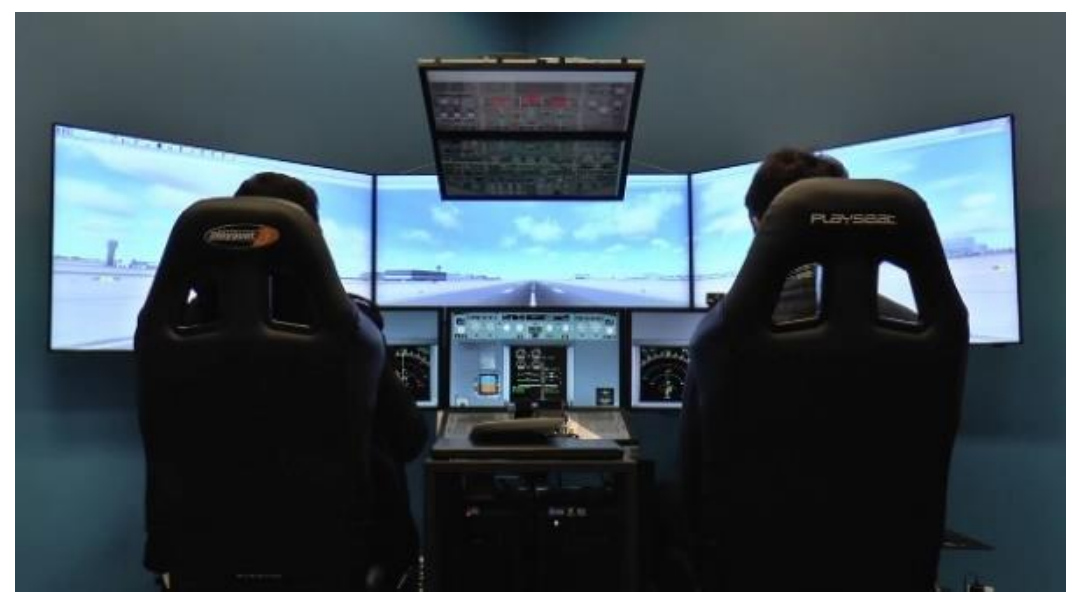

FIGURE 68: THE SYMBOLOGY TESTING WAS PERFORMED ON THE RYERSON FIXED BASE SIMULATOR (FBS) [53] WHILE THE TEXTURE TESTING WAS PERFORMED ON PRESAGIS HELICRAFT.

\section{FLIGHT PLAN}

The testing calls for a full flight envelope to be performed, a takeoff, maneuver, cruise, approach, and landing are all performed. For the test case, a takeoff is performed at the Aden City airport, Yemen and fly towards the Aden City stadium in the outskirts of the city to perform a landing at the center of a soccer field as illustrated in Figure 69. The following flight plan was chosen to test specific helicopter operations in multiple scenarios along with testing pilot capability. The flight plan is followed by an explanation of the reason specific routes and maneuvers were chosen.

1. Take off at Aden City Airport (Runway) and maintain hover close to 0 knots ground speed and $40 \mathrm{ft}$ radar altitude for 10-15 seconds. Use the Runway markings and lines to position self at hover visually.

2. Increase altitude and ground speed to reach end of runway maintaining a course of 80 degrees.

3. At the end of the runway bank/turn (about turn) to the right to course 250 degrees.

4. Maintain course and fly parallel to the runway and bank/turn right to course 360 degrees and achieve visual of the stadium.

5. Maintain course and altitude below $500 \mathrm{ft}$ radar and speed no greater than 100 knots (airspeed).

6. Continuously maintain high reconnaissance of the landing area, which is the stadium.

7. Fly to the stadium while slowing down as required to keep visual contact of landing target OTW.

8. Once over the stadium maintain hover and constant heading (approx. True North, 360 Deg.)

9. Maintain hover over the soccer field and hover down slowly.

10. Use the 2D symbology and 3D symbology as required. 
11. For the test involving the 3D symbology, use the waypoint marker to navigate and reach landing zone.

The stated flight plan was selected to highlight all the problems specific to the helicopter operating environment from a pilot perspective. The chosen landing zone/point is at the center of a soccer stadium which requires the participants to maneuver the helicopter into the landing zone accurately. This scenario allows for the testing of how much symbology helps in landing. The test subjects will be asked to follow the flight plan in three different scenarios. The first scenario is operating without the $2 \mathrm{D}$ symbology, and a representation of present-day operating scenarios pilots use, with flight instrumentation such as a PFD. The second scenario is operating with the 2D symbology (i.e., Hover and Cruise symbologies), the pilots would be asked to use the $2 \mathrm{D}$ symbology to assist them in flying the flight plan. The third test case utilizes the 2D symbology and the 3D (conformal) symbology to assist the pilot in completion of the task. As shown in Figure 69, the chart map layout shows the flight plan described above.

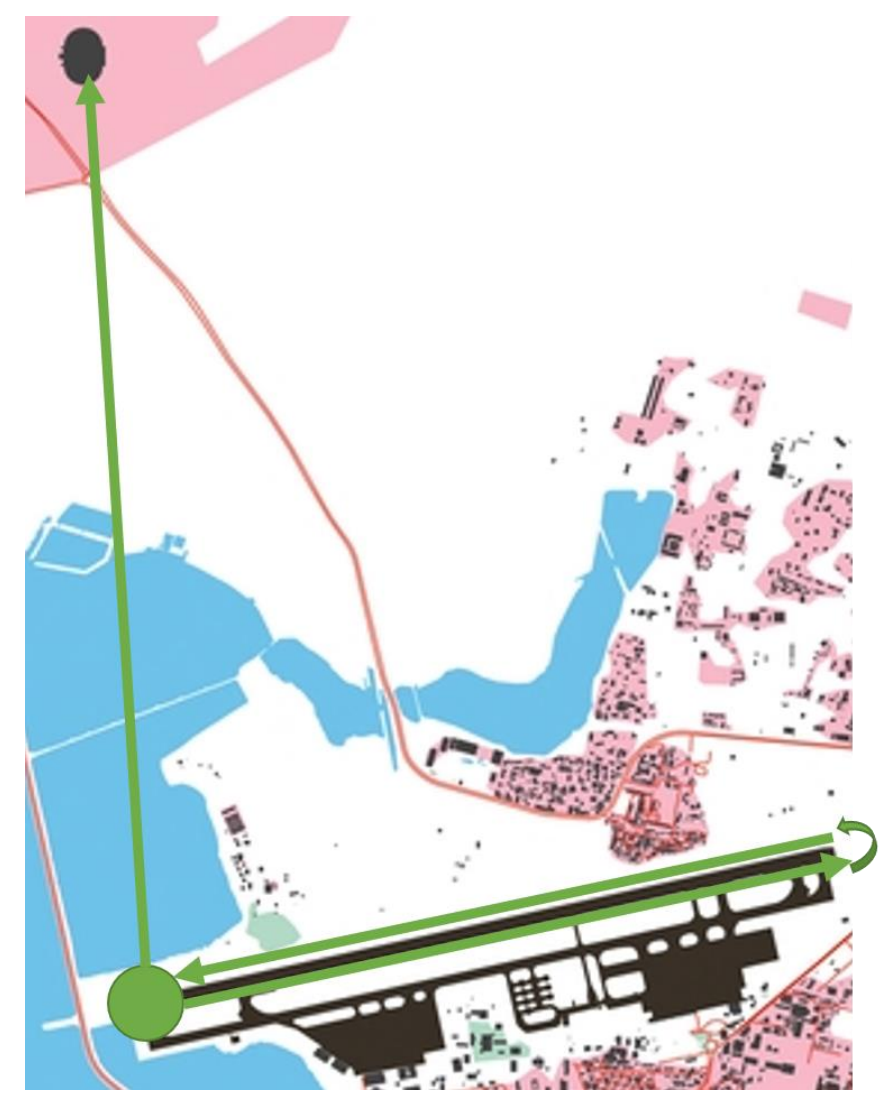

FIGURE 69: MAP OF THE FLIGHT PLAN, SHOWING ROUTE TO BE FOLLOWED BY THE PARTICIPANTS.

\section{TERRAIN TEXTURE}

The following test scenario was used to shortlist the textures. The ideal texture should be able to help perceive the attitude of terrain in several operating conditions. The texture should be able to provide the 
necessary perception in low altitude, high altitude flight, varying degrees of slopes, and terrain elevations. The number of participants available for testing terrain textures is smaller due to the higher complexity of flying required and detail in testing required. Two participants with higher flight (helicopter) experience were tasked to obtain metrics on texture usability. The following maneuvers were requested, and participants replied with the either "great" "good" "nominal" "poor" and "bad" for what they perceived with respect to the requested flight maneuver. Such a rating scale was a better approach since it did not put pressure on the pilots to choose an exact perceived value or a range while flying. The camera angle was at 30 degrees down, and there was always a bias in the value being ill estimated. Providing descriptors was a better option since it was more efficient than the pilot trying to correlate the number they perceive. A more significant emphasis was based on a low-level flight than higher altitude due to the goal being addressed is based on landing, hovering and low-level flight maneuvers.

1. A level overflight for 20 seconds of a flat region near the mountains at $100 \mathrm{ft}$ AGL and 100 knots.

2. Same as 1 but vary the altitude to $50 \mathrm{ft}$ and $10 \mathrm{ft}$.

3. Same as 1 but vary the terrain texture.

4. Same as 1 but vary the speed to 50 knots, 30 knots, 10 knots and 0 knots. A significant component of the flight testing was the testing of textures for use in different conditions. Two test subjects were available, as the testing called for more experienced pilot who were familiar with understanding and reading from the terrain. The terrain information must be used to gather speed/drift altitude and attitude information. A participant with longer rotorcraft flying experience and ability to distinguish subtle terrain changes are important. Two participants with greater than 100 and 80 hours of rotorcraft flying experience (simulation) were requested for flight testing of terrain textures. They were asked to perform take-offs and maintain precise flight parameters. Once attaining the parameters requested the participants were asked to rate in terms of Bad (1), Poor (2), Fair (3), Good (4) and Excellent (5).

\section{D SYMBOLOGY}

The purpose of 2D symbology development was to provide precise correlation and information of the aircraft with relation to the environment. The test criteria would call for testing of both phases, Hover and Cruise symbology. Though there are many components, the major tested articles are those that provide the necessary situational awareness to the participant. This test case assumes participants only have access to the cruise and hover symbology to perform the necessary landing. Referring to chapter 2.3 on pilot operations, a pilot must perform a high and low reconnaissance and approaches to land at the soccer field where they encounter a brownout. The participant must land the helicopter at the center of the soccer field.

\section{HOVER}


The IIMC conditions force the pilot to perform the following maneuvers and for testing the cases necessary for this study.

1. Using the $2 \mathrm{D}$ symbology is the pilot able to enter the landing zone premises at high altitude and descend to low hover over the stadium?

2. Hover above the chosen LZ and perform a descent facing a north end to promote easier exfiltration.

3. The pilot should be able to rely on the $2 \mathrm{D}$ hover symbology, turn and rotate and maneuver within the soccer field while in low hover.

Thus, the pilots would initially look to land the aircraft looking out the window and then with the Hover display. Their response on their performance and what they felt would be recorded in a means to understand the improvement in performance. The pilot may use any visual cues exterior to the cockpit that are available to them.

\section{CRUISE}

In the cruise condition, the pilots would be tasked to take off and fly from the airport to descent portion of the landing. The participants will use the same flight path mentioned above, and they will be provided with the flight plan, which they will be asked to follow based on the available symbology in the cruise component. The pilots were thoroughly provided with a mission briefing which they shall follow on the simulator using the available cruise symbology.

\section{D SYMBOLOGY}

The purpose of the 3D symbology is to allow for landing while acting as conformal environmental objects. The pilots would use these to position the aircraft in place and land it, as a normal pilot operating under VMC would.

1. The participants would be asked to use the 3D symbology to perform a landing completely based on virtual VMC cues attained from using the 3D symbology.

2. In addition to the landing, the pilots would be asked to maintain hover utilizing the 3D symbology.

3. Ability to perceive helicopter drift from the symbology.

4. Ability to land precisely on the landing point.

5. Ability to understand the orientation and direction of landing.

The 3D symbology component is also composed of waypoint markers placed conformally in the terrain acting as a navigation method to direct pilots to a landing zone. 
The overall test plan aims to measure the workload, stresses, and capabilities the participants felt during the testing process. 8 participants were requisitioned to fly the selected flight path and land inside a stadium, using three different symbology sets via a generic PFD, 2D and 3D symbology. Moments of the flight plan were analyzed to see how the accuracy of flight changes with using the three different symbology sets.

During the flight of each individual participant, data was collected with respect to various flight parameters that would allow the observation of flight stability. The following information was collected in real time and plotted

1. Time

Allows to see how the time differences are in the landings with the three different symbology sets.

2. Altitude

Provides an insight into the changes in altitude, allows for judging how well the participant was able to perform station-keeping duties with the display. Pilots would not be able to obtain precise altitude understanding looking out the window, thus providing altitude restrictions forces station keeping jobs. The accuracy in the altitude can be used to understand how the displays facilitated for good station-keeping ability.

3. Velocity in $\mathrm{X}$ (Longitudinal)

Observance of the Velocity in the longitudinal direction allows how stable/unstable pilot accelerations and decelerations are in achieving landing. The trends will be observed for erratic oscillations to see how often pilots had to make corrections. In an optimal scenario, the pilot would make a smooth decelerating approach and landing. This can, however, change when the pilot is not sure or confused with their operating speeds and locations.

4. Velocity in Y (Lateral)

Allows for the observation of the velocity in the lateral direction, can understand how stable/unstable the pilot accelerations and decelerations are in achieving landing. The trends will be observed for lateral stability to observe how much the pilot had to correct based on display information. In an optimal scenario, once a pilot chooses the approach direction, their landings are straight with minimal lateral corrections. This parameter will be used to observe how the pilot would correct laterally.

5. Velocity in Z (Vertical)

Allows for the observation of the changes in vertical velocity, when close to landing Vertical Velocity parameter can be used to observe how much the pilot had to correct themselves vertically. This allows for observation of changes in altitude, and pilots 
tend to use external cues for judging altitude and vertical velocity. Thus, this parameter would prove to be effective in discretizing the help provided by the display in providing enough information to the pilot about the altitude and vertical velocity.

The collected data can be utilized to observe how stable a participant flies with three different display systems. Each of the three displays shows an array of information that the pilot would need. Thus, it is effective to observe how the pilot uses this information and how it helps them land more effectively and safely. This collected information can also be used to verify and compare the responses qualitatively from participants on the workloads, display ratings and NASA TLX demands on the mental, physical, temporal, frustration levels experienced.

\section{TEST PARTICIPANTS}

The participants in the flight test were all students known to the primary investigator and have varied flight experience, simulated and real flight experience. The participant subjects have limited flight experience with helicopter operations. The participants were trained in maintaining a hover and flying characteristics that would allow them to perform for the designed flight test. Students had to be taught on flying the helicopter simulator as an experienced pilot were unavailable for testing purposes. Table 6 summarizes the participant flight and simulation experience. All participants are volunteers; recruiting was done via email and recruitment letters. The Ryerson Ethics Board (REB) approval was obtained for human testing.

TABLE 6: BREAKDOWN OF PARTICIPANT EXPERIENCE INFORMATION PRIOR TO FLIGHT TESTING.

\begin{tabular}{|c|c|c|c|}
\hline Participant \# & Flight hrs (\#) & Fixed wing simulator & Helicopter Sim Experience \\
\hline Participant 1 & N/A & $15 \mathrm{hrs}$ & $<5 \mathrm{hrs}$ \\
\hline Participant 2 & N/A & $100 \mathrm{hrs}$ & $<5 \mathrm{hrs}$ \\
\hline Participant 3 & N/A & $20 \mathrm{hrs}$ & $<5 \mathrm{hrs}$ \\
\hline Participant 4 & N/A & $50 \mathrm{hrs}$ & $<5 \mathrm{hrs}$ \\
\hline Participant 5 & N/A & $6 \mathrm{hrs}$ & $<5 \mathrm{hrs}$ \\
\hline
\end{tabular}


Participant 6

Participant 7

Participant 8

Participant 9
N/A

$50 \mathrm{hrs}$

N/A

N/A
$55 \mathrm{hrs}$

$60 \mathrm{hrs}$

$40 \mathrm{hrs}$

100
$<5 \mathrm{hrs}$

$<7 \mathrm{hrs}$

$<80 \mathrm{hrs}$

$<100 \mathrm{hrs}$ 


\subsection{TABULATION AND EVALUATION OF RESULTS}

As described in Chapter 5.1, several flight parameters were collected in real-time, that can be used to judge the participant performance with different display types. Thus, in the following chapter, the vertical, longitudinal, lateral are compared between the three different display types to understand how the performance has improved in the flight regime. Each figure of the graphed parameter concerning the display is explained with the individual conclusion being drawn. The results from flight tests and participant responses are substantiated by landing accuracy of the helicopter to the requested landing spot. This result can be used to judge how the displays improved landing accuracy and performance.

The first measured flight parameter analyzed is the initial hover case requested from the participants in the flight plan. In the first step of the flight plan, the participant was asked to take off at the runway and maintain hover for about ten seconds. The vertical velocity, altitude, and Longitudinal velocities will be observed to understand and distinguish accuracy between the three displays. The testing consisted of 8 participants. Thus 24 different figures will be used to describe the mentioned parameters among of the three displays eight participants and three observed flight parameters. The performance of each participant is discussed below.

The most important parameter to define if a helicopter is in hover or not is the longitudinal/lateral speed of the helicopter, the closer it is to zero the closer the helicopter is to a maintained hover. Observing the longitudinal/lateral speed of the helicopter the maintenance of hover can be attested.

Three displays were tested, using a generic PFD display, 2D symbology variation, and Virtual-VFR (3D and 2D) display. The participants were asked to maintain a 10-second hover for the first two flight tests, using a PFD and 2D symbology. For the Virtual-VFR symbology, the hover case was relaxed as the same 2D symbology was overlaid on top of the Virtual-VFR display. Thus, the virtual VFR (labeled 3D DVE) results for hover are not shown.

Followed by results of the hover test condition, participant submitted non-intrusive questionnaire results would be presented and discussed which highlight the mental, physical, temporal demands and frustration, level of effort and self-evaluation of the performance of task completion. The questionnaires also capture the workload, stress and experienced by the participants.

Finally, the accuracy of landing points is an essential indicator of how the displays improve landing accuracy and how they allow for mission re-planning. All participants were requested to land at the center of the stadium, thus, based on where participants landed using the symbology can provide information on how the accuracy has improved using symbology. 


\section{FLIGHT TEST DATA}

TABLE 7: INITIAL HOVER PHASE DATA FOR PARTICIPANTS 1 THROUGH 8.

\begin{tabular}{|c|c|c|}
\hline \multirow[t]{3}{*}{ Altitude (Avg-ft) } & Lateral Velocity Vy & Longitudinal \\
\hline & $(A v g-$ & Velocity Vx (Avg- \\
\hline & Knots)/requested & knots) /requested \\
\hline
\end{tabular}

Time Required $(\mathrm{Sec})$

(P1) PFD Display

$50 \mathrm{ft} / 40 \mathrm{ft}$

$-3.5 \mathrm{knts} / 0 \mathrm{knts}$

$2 \mathrm{knts} / 0 \mathrm{knts}$

Altitude: 40

Vz: 25, Vx: 25

(P1) 2D Display

$43 \mathrm{ft} / 40 \mathrm{ft}$

$1 \mathrm{knts} / 0 \mathrm{knts}$

$1.75 \mathrm{knts} / 0 \mathrm{knts}$

Altitude: 40

Vz: 15, Vx: 15

(P1) 3D Display

N/A

N/A

N/A

N/A

(P2) PFD Display

$42 \mathrm{ft} / 40 \mathrm{ft}$

$1.0 / 0(\mathrm{ft} / \mathrm{s})$

$1 \mathrm{knts} / 0 \mathrm{knts}$

Altitude: 25

Vz: 18, Vx: 18

(P2) 2D Display

$35 \mathrm{ft} / 40 \mathrm{ft}$

$0.5 / 0(\mathrm{ft} / \mathrm{s})$

2 knts/0 knts

Altitude: 15

Vz: 18, Vx: 18

(P2) 3D Display

N/A

N/A

N/A

N/A

(P3) PFD Display

$30 \mathrm{ft} / 40 \mathrm{ft}$

$1.0 / 0(\mathrm{ft} / \mathrm{s})$

3 knts/0 knts

Altitude: 15

Vz: 18, Vx: 15

(P3) 2D Display

$40 \mathrm{ft} / 40 \mathrm{ft}$

$1.0 / 0(\mathrm{ft} / \mathrm{s})$

$1 \mathrm{knts} / 0 \mathrm{knts}$

Altitude: 15

Vz: 18, Vx: 15

(P3) 3D Display

N/A

N/A

N/A

N/A

(P4) PFD Display

$30 \mathrm{ft} / 40 \mathrm{ft}$

$4.0 / 0(\mathrm{ft} / \mathrm{s})$

$12 \mathrm{knts} / 0 \mathrm{knts}$

Altitude: 30

(P4) 2D Display

$40 \mathrm{ft} / 40 \mathrm{ft}$

$2.5 / 0(\mathrm{ft} / \mathrm{s})$

$10 \mathrm{knts} / 0 \mathrm{knts}$

Vz: 45, Vx: 30

(P4) 3D Display

N/A

N/A

N/A

N/A 
(P5) PFD Display

Altitude: 18

$25 \mathrm{ft} / 40 \mathrm{ft}$

$4.0 / 0(\mathrm{ft} / \mathrm{s})$

2 knts/0 knts

Vz: 18, Vx: 45

(P5) 2D Display

Altitude: 10

$38 \mathrm{ft} / 40 \mathrm{ft}$

$2.5 / 0(\mathrm{ft} / \mathrm{s})$

1 knts/0 knts

Vz: 12, Vx: 30

(P5) 3D Display

N/A

N/A

N/A

N/A

(P6) PFD Display

$30 \mathrm{ft} / 40 \mathrm{ft}$

$2.0 / 0(\mathrm{ft} / \mathrm{s})$

$4.0 \mathrm{knts} / 0 \mathrm{knts}$

Altitude: 15

(P6) 2D Display

$40 \mathrm{ft} / 40 \mathrm{ft}$

$1.0 / 0(\mathrm{ft} / \mathrm{s})$

N/A

(P6) 3D Display

N/A

(P7) PFD Display

$50 \mathrm{ft} / 40 \mathrm{ft}$

$4.0 / 0(\mathrm{ft} / \mathrm{s})$

$2.5 \mathrm{knts} / 0 \mathrm{knts}$

Vz: 30, Vx: 20

(P7) 2D Display

$45 \mathrm{ft} / 40 \mathrm{ft}$

$2.5 / 0(\mathrm{ft} / \mathrm{s})$

$1.0 \mathrm{knts} / 0 \mathrm{knts}$

Altitude: 10

(P7) 3D Display

N/A

N/A

N/A

N/A

(P8) PFD Display

$41 \mathrm{ft} / 40 \mathrm{ft}$

6.0/0 (ft/s)

$5.0 \mathrm{knts} / 0 \mathrm{knts}$

Altitude: 15

(P8) 2D Display

$41 \mathrm{ft} / 40 \mathrm{ft}$

$3.0 / 0(\mathrm{ft} / \mathrm{s})$

$1.0 \mathrm{knts} / 0 \mathrm{knts}$

Vz: 15, Vx: 5

(P8) 3D Display

N/A

N/A

N/A

N/A 


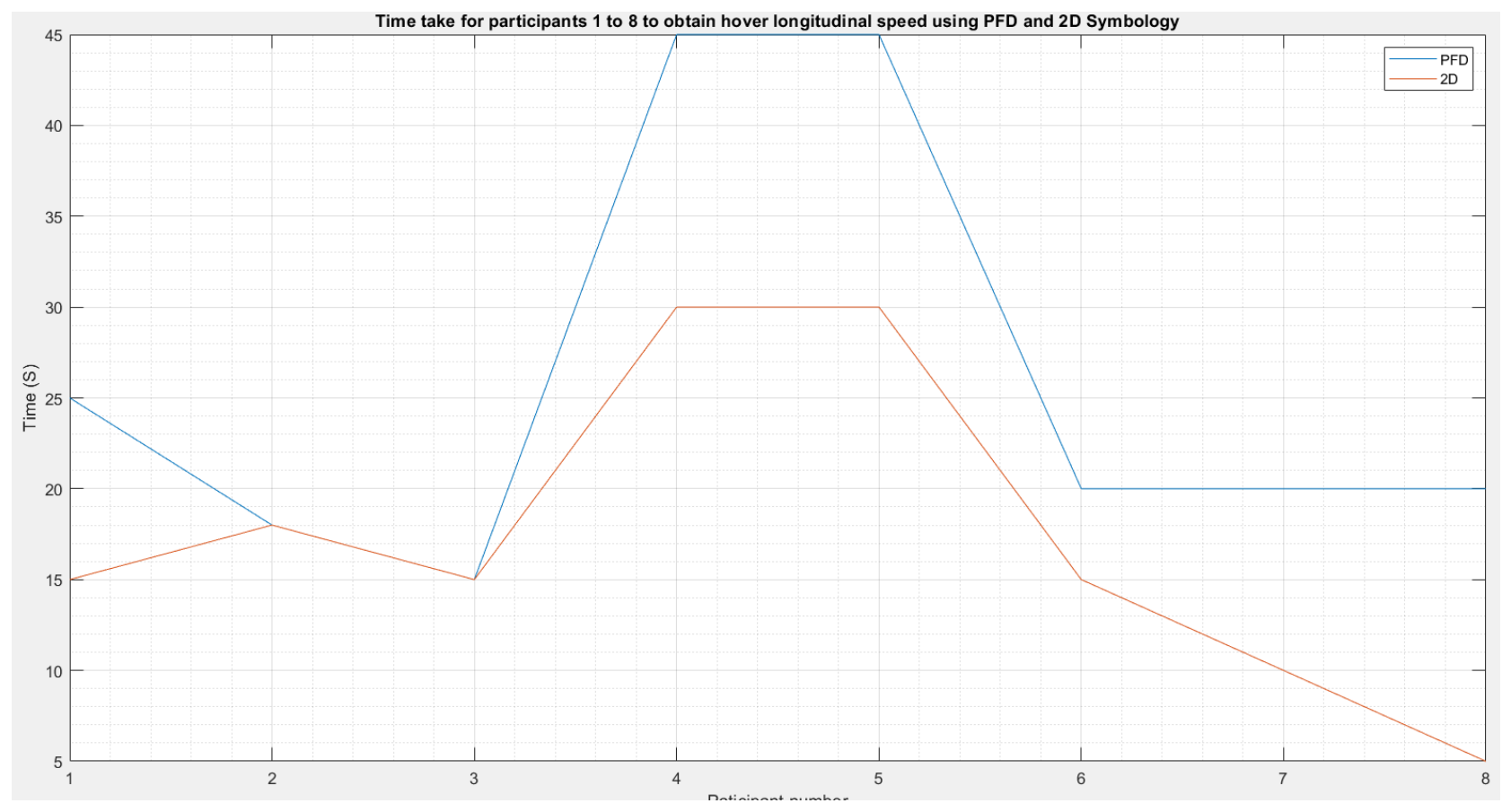

FIGURE 70: PLOT OF TIME TAKEN TO ACHIEVE HOVER LONGITUDINAL SPEED BY PARTICIPANTS 1 THROUGH 8 WITH THE PFD AND 2D DISPLAYS.

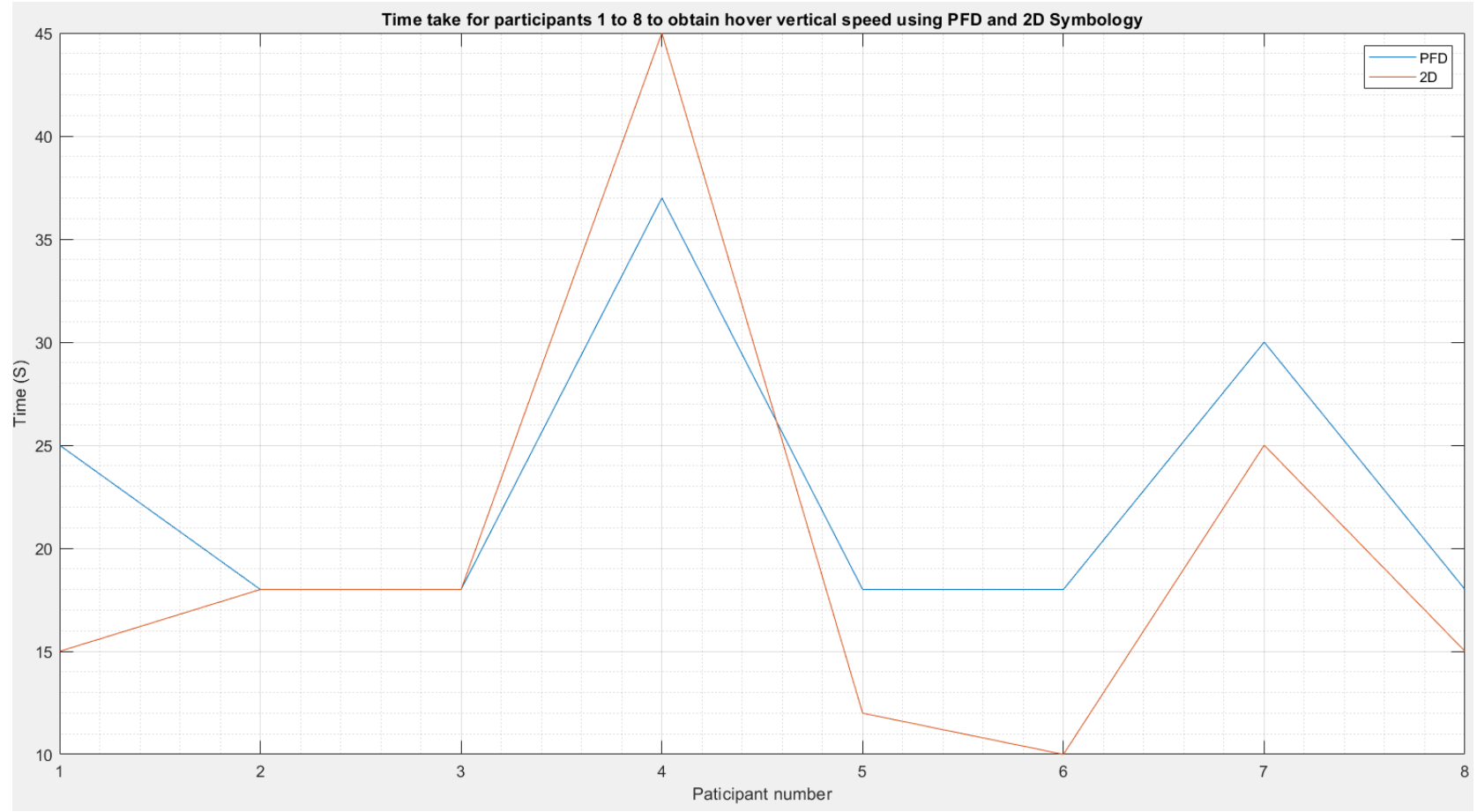

FIGURE 71: PLOT OF TIME TAKEN TO ACHIEVE HOVER VERTICAL SPEED BY PARTICIPANTS 1 THROUGH 8 WITH THE PFD AND 2D DISPLAYS. 


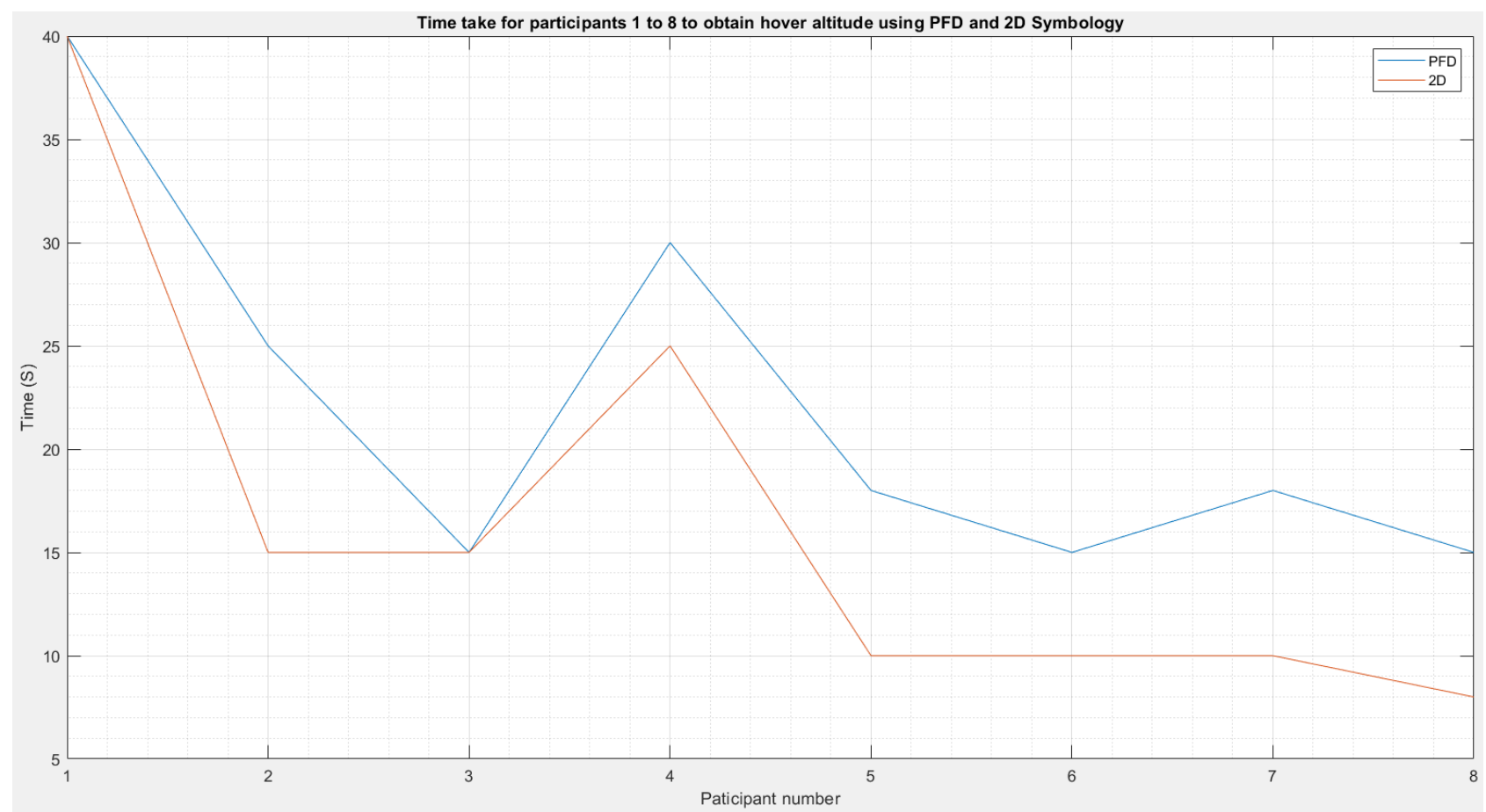

FIGURE 72:PLOT OF TIME TAKEN TO ACHIEVE HOVER VERTICAL SPEED BY PARTICIPANTS 1 THROUGH 8 WITH THE PFD AND 2D DISPLAYS.

Figures 70, 71 and 72 visualize the data summarized in Table 7, the time taken to achieve hover longitudinal speed, hover vertical speed and hover altitude prescribed. These figures are present to visualize the data (Table 7) and the discussion below.

\section{Participant - 1}

In the collected data from the flight test, the first participant is analyzed in two different phases, initially a hovering phase and then the landing phase. In the initial phase, where a hover was requested upon take-off, it was observed for participant one; the hover was achieved faster with the use of orthographic (Represented as 2D) DVE symbology. The hover was achieved about 10 seconds quicker with the $2 \mathrm{D}$ symbology over the PFD. After observing the lateral and longitudinal velocity, the average drift speeds were higher for the PFD as opposed to the 2D orthographic symbology. Thus, using the 2D orthographic symbology provided for more accurate maintenance of requested hover condition, with an accurate hover profile and accuracy of requested altitude. Table 7 contains the data of participant one's hover for the PFD, 2D, and 3D displays.

\section{Participant -2}

The second participant from the flight test showed that a more accurate flight was maintained with the 2D display, with minimal drifts in the vertical and longitudinal directions and very close to requested altitude. The times taken to achieve the required hover conditions show the 2D display performing better to its PFD counterpart. The longitudinal speed was slightly higher for the 2D display. Table 7 contains the data of participant two's hover for the PFD, 2D, and 3D displays. 


\section{Participant -3}

The tests conducted with the third participant showed the results to be similar for both PFD and 2D orthographic displays. The times required to achieve hover (altitude, vertical velocity, and longitudinal velocity) were similar. The vertical velocity on average was the same with both displays, and the longitudinal drift velocity was higher for the PFD display (3ft/s) over the 2D orthographic display (1ft/s). The altitude maintained on average was very accurate to requested altitude value with the $2 \mathrm{D}$ orthographic symbology. Table 7 contains the data of participant three's hover for the PFD, 2D, and 3D displays.

\section{Participant - 4}

The fourth participant's flight test results showed the 2D orthographic symbology performing better to its PFD counterpart. Using the PFD display, the participant achieved to maintain an altitude of 30ft while maintaining the required $40 \mathrm{ft}$ altitude requested with the $2 \mathrm{D}$ orthographic display. The lateral and longitudinal drift velocities were also smaller with the 2D orthographic display compared with PFD display. The time taken to maintain the requested conditions were quicker with the $2 \mathrm{D}$ orthographic display. Overall the $2 \mathrm{D}$ orthographic data proved to be of better at maintaining a less erratic hover. Table 7 contains the data of participant four's hover for the PFD, 2D, and 3D displays.

\section{Participant -5}

The fifth participant's data showed similar results to the other participants, with an overall performance recorded to be better with the $2 \mathrm{D}$ orthographic display. More accurate altitude to the requested was maintained with the $2 \mathrm{D}$ display, on average a $38 \mathrm{ft}$ of $40 \mathrm{ft}$ requested, while with the $\mathrm{PFD}$, an average altitude of $25 \mathrm{ft}$ was maintained. Similarly, there were more significant drifts/deviations in the vertical and longitudinal directions with the PFD compared to 2D orthographic display. The time taken to obtain the requested altitude, vertical and longitudinal drift velocities were attained faster with the $2 \mathrm{D}$ orthographic symbology. Table 7 contains the data of participant five's hover for the PFD, 2D, and 3D displays.

\section{Participant - 6}

The results from the flight test of participant six showed consistent results with the other participants. The results highlight a hovering phase that was achieved faster and exact maintenance of altitude at 40ft, the vertical and longitudinal drift velocities were also smaller for the 2D orthographic symbology compared to the PFD display. Table 7 contains the data of participant six's hover for the PFD, 2D, and 3D displays.

\section{Participant - 7}

The results from the flight test of participant seven showed consistent results with the other participants. The results highlight a hovering phase that was achieved faster and closer maintenance of altitude at $45 \mathrm{ft}$ 
to the requested $40 \mathrm{ft}$, the vertical and longitudinal drift velocities were also smaller for the $2 \mathrm{D}$ orthographic symbology compared to the PFD display. Table 7 contains the data of participant seven's hover for the PFD, $2 \mathrm{D}$, and 3D displays.

\section{Participant - 8}

The results from the flight test of participant eight showed consistent results with the other participants. The results highlight a hovering phase that was achieved faster. The altitude maintained was the same for both display types (PFD and 2D orthographic) at 41ft. The vertical and longitudinal drift velocities were smaller for the $2 \mathrm{D}$ orthographic symbology compared to the PFD display. Table 7 contains the data of participant eight's hover for the PFD, 2D, and 3D displays.

\subsection{NON-INTRUSIVE QUESTIONNARIE RESULTS}

The following section presents the information gathered from non-intrusive questionnaires, the NASA TLX frustration, self-performance, effort, temporal, physical and mental demand ratings for the PFD display, 2D Orthographic display and 3D DVE display. In Figures 73 to 80, the green bars represent the individual responses while the red bars represent the average value of participant response.

\section{NASA TLX}

\section{Frustration Experienced}

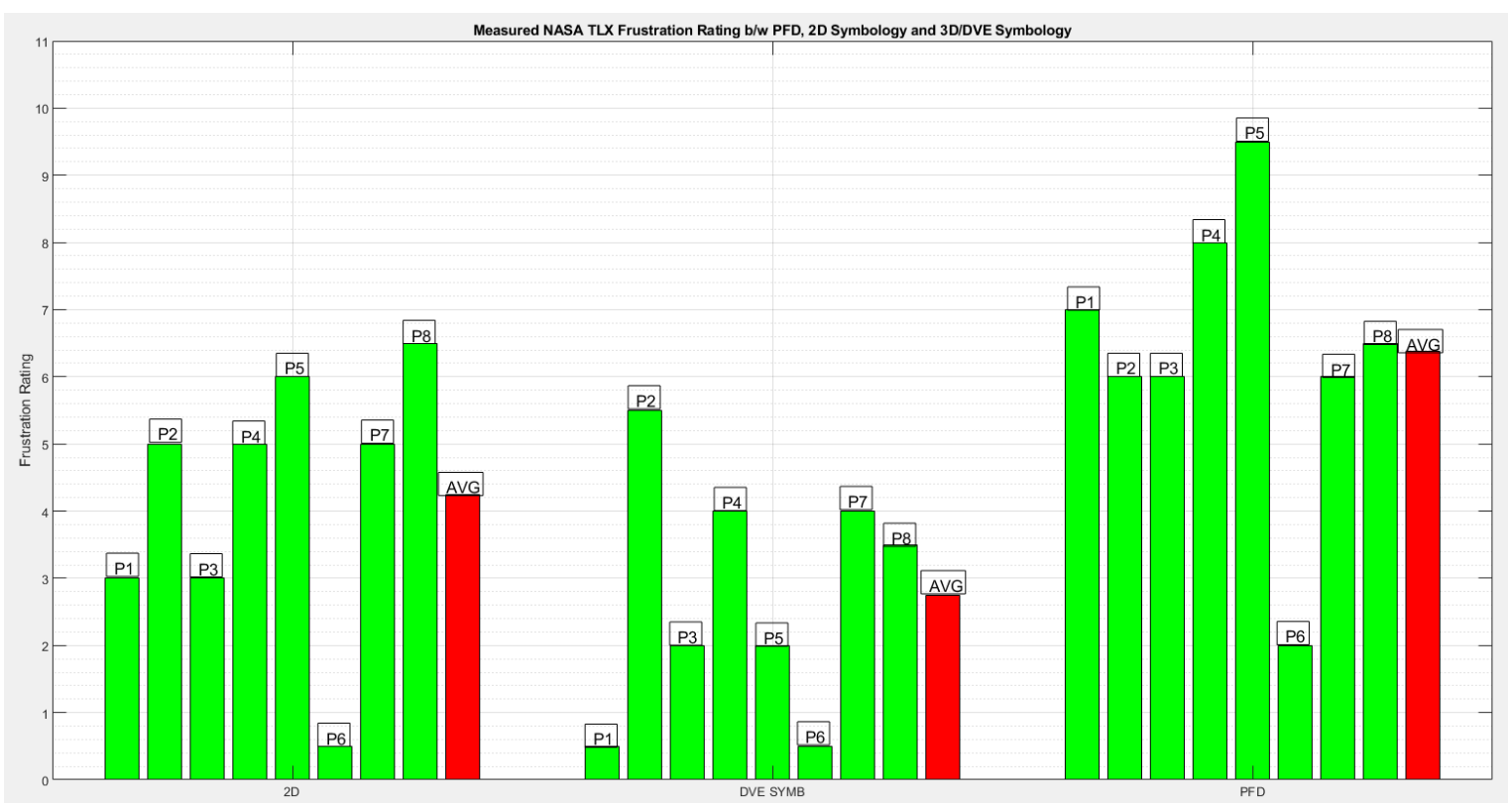

FIGURE 73: FRUSTRATION EXPERIENCED SURVEY RESULTS BY PARTICIPANTS USING PFD, 2D AND 3D DISPLAYS FROM NASA TLX FRUSTRATION SCALE. 
The participants reported through the NASA TLX rating scale for the frustration that on average, the frustration level decreased when going from using the PFD display to the $2 \mathrm{D}$ orthographic display to the 3D DVE symbology. As can be seen in Figure 73, in the PFD response, the average frustration experienced was 6.4 or $64 \%$. The $2 \mathrm{D}$ orthographic response highlights the average frustration value experienced was $43 \%$, showing a decrease in the frustration by about $20 \%$. Similarly, using the 3D DVE symbology, the average frustration decreased to $27 \%$, showing a significant decrease in the frustration experienced by participants who were using the PFD display to fly and land.

\section{Self-Performance rating}

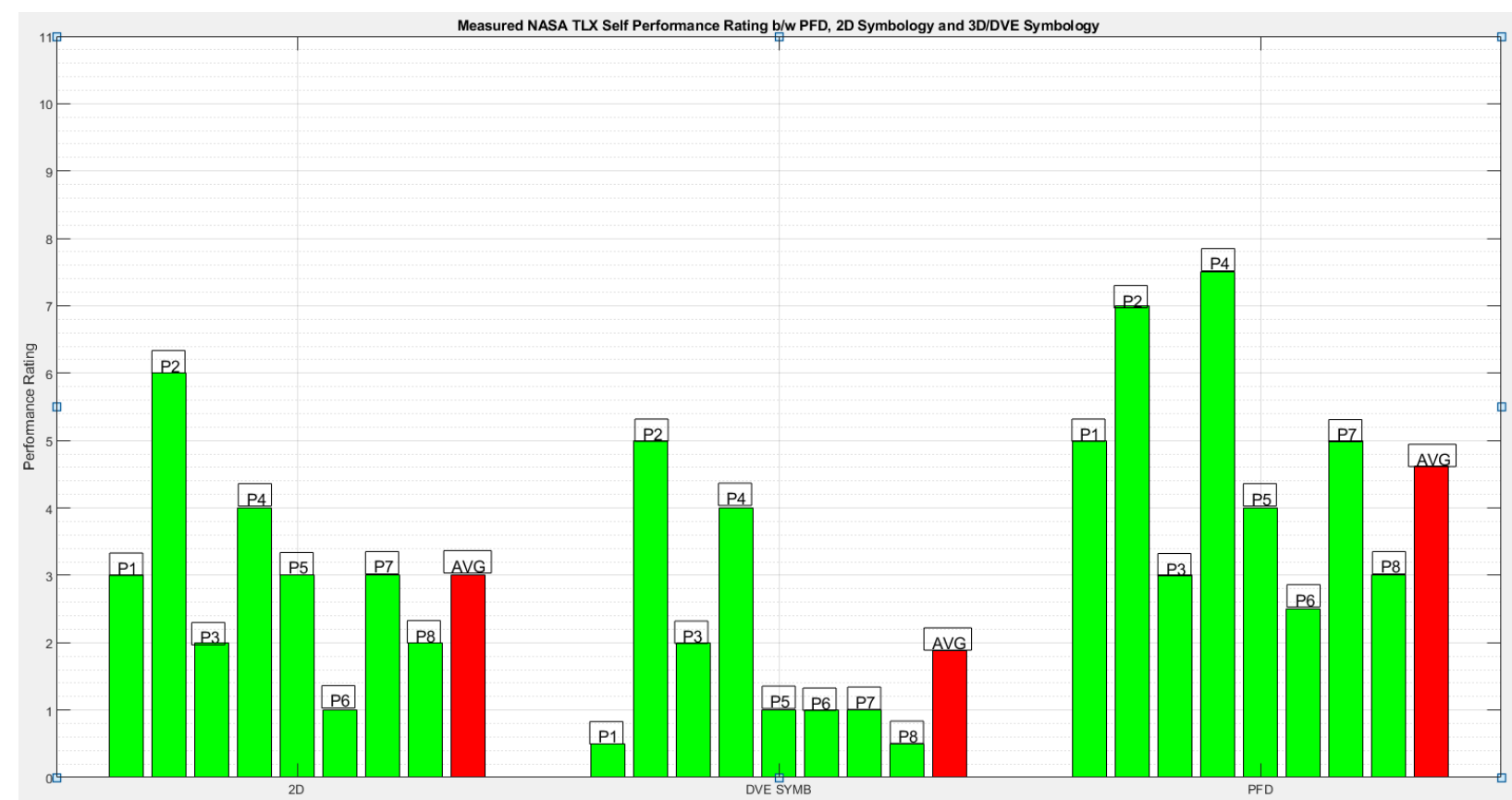

FigURE 74: SELF PERFORMANCE SURVEY RESULTS BY PARTICIPANTS USING PFD, 2D AND 3D DISPLAYS FROM NASA TLX SELF-PERFORMANCE SCALE.

The rating scale for self-performance is reversed to the other scales on the NASA TLX rating scale, the lower the number, the better it is. Suppose a rating of ' 1 ' means the participant rated their performance as 'Good' a rating of '10' means 'Poor.' The participants reported through the NASA TLX rating scale for self-performance that on average, their performance level increased when going from using the PFD display to the 2D orthographic display to the 3D DVE symbology. As can be seen in Figure 74, in the PFD response, the average self-performance rating was 4.3 or $43 \%$. The 2D orthographic response highlights the average performance value experienced was $30 \%$, showing an increase in performance by about $13 \%$. Similarly, using the 3D DVE symbology, the average performance was 19\%, showing a significant performance increase in participants who were using the $3 \mathrm{D}$ symbology and 2D symbology display to fly and land over the PFD. 


\section{Effort Required}

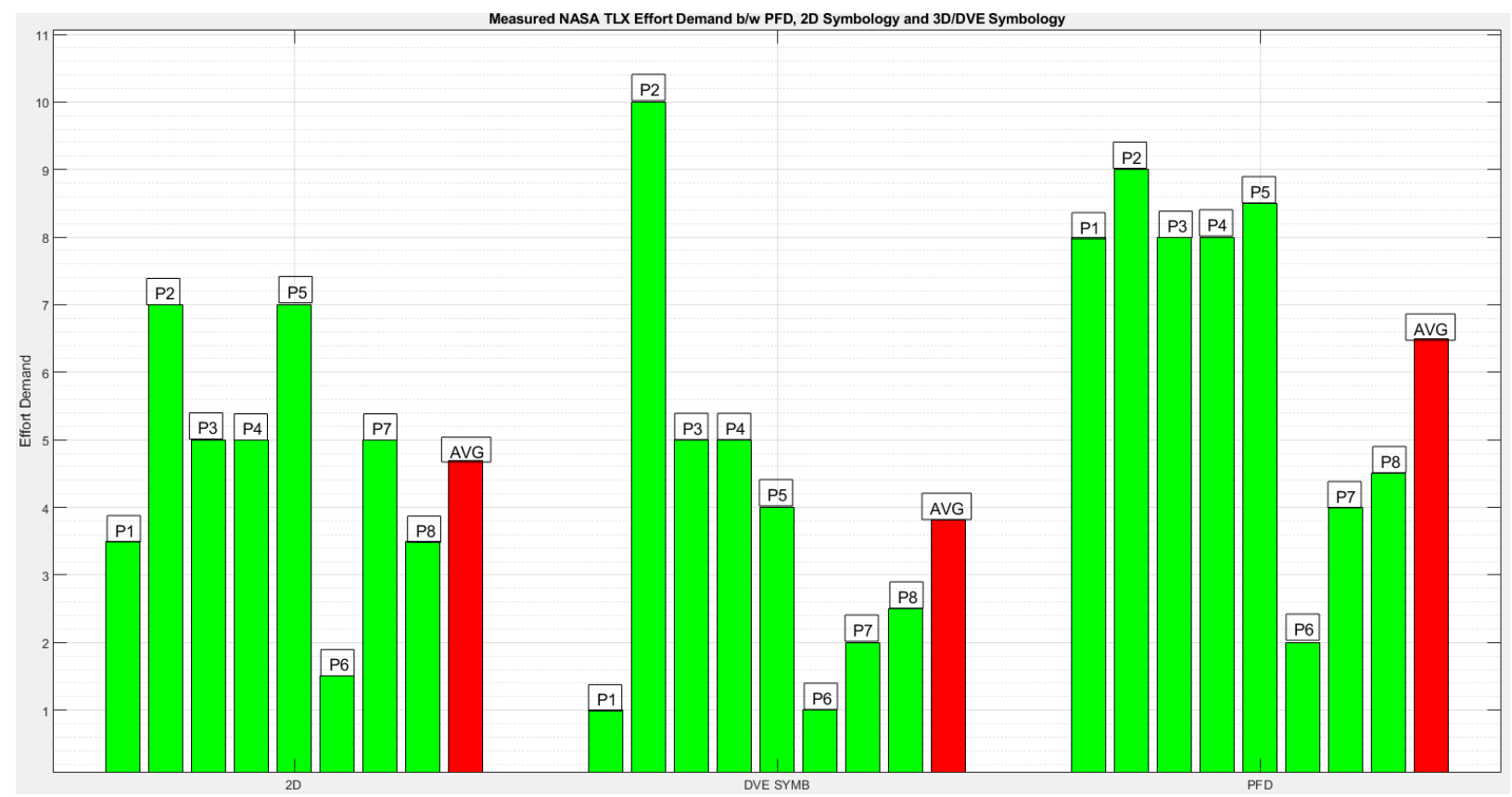

FIGURE 75: EFFORT REQUIRED SURVEY RESULTS BY PARTICIPANTS USING PFD, 2D AND 3D DISPLAYS FROM NASA TLX EFFORT SCALE.

The participants reported through the NASA TLX rating scale for the effort required that on average, the effort level decreased when going from using the PFD display to the 2D orthographic display to the 3D DVE symbology. As can be seen in Figure 75, in the PFD response, the average effort experienced was $65 \%$. The 2D orthographic response highlights the average effort value experienced was $47 \%$, showing a decrease in the required effort by about $20 \%$. Similarly, using the 3D DVE symbology, the average effort decreased to $38 \%$, showing a significant decrease in the effort experienced by participants who were using the PFD display to fly and land. 


\section{Temporal Demand}

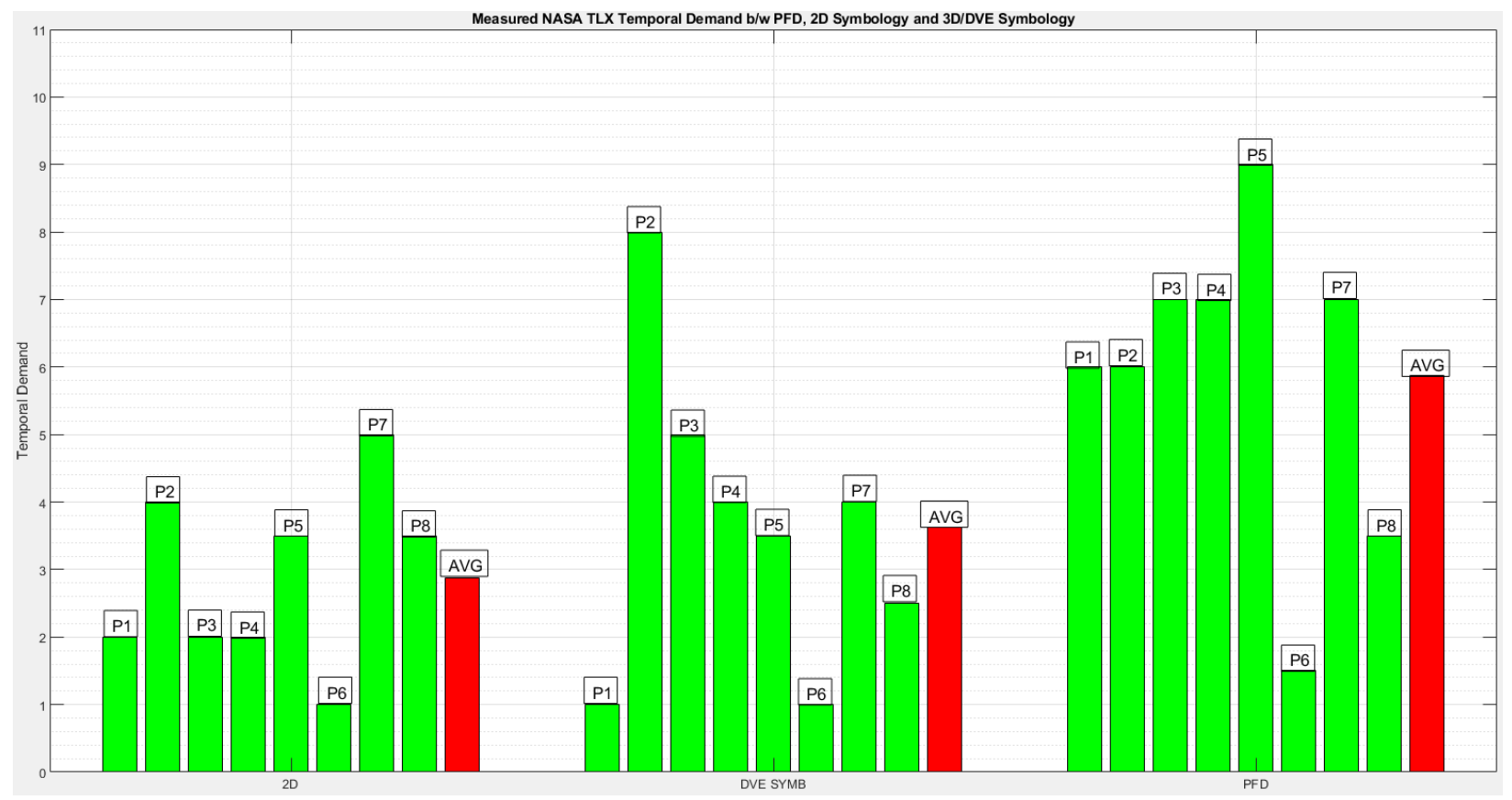

FIGURE 76: TEMPORAL DEMAND EXPERIENCED SURVEY RESULTS BY PARTICIPANTS USING PFD, 2D AND 3D DISPLAYS FROM NASA TLX TEMPORAL DEMAND SCALE.

The participants reported through the NASA TLX rating scale for temporal demand required that on average the temporal demand level decreased and then increased when going from using the PFD display to the 2D orthographic display to the 3D DVE symbology. As can be seen in Figure 76, in the PFD response, the average temporal demand experienced was $59 \%$. The 2D orthographic response highlights the average temporal demand value experienced was $29 \%$, showing a decrease in the required effort by about $30 \%$. Similarly, using the 3D DVE symbology, the average temporal demand increased to $36 \%$, showing a significant decrease in the temporal demand experienced by participants who were using the PFD display to fly and land. The temporal demand decreased between the PFD display and 2D orthographic display and increased between the 2D orthographic display and 3d DVE display. 


\section{Physical Demand}

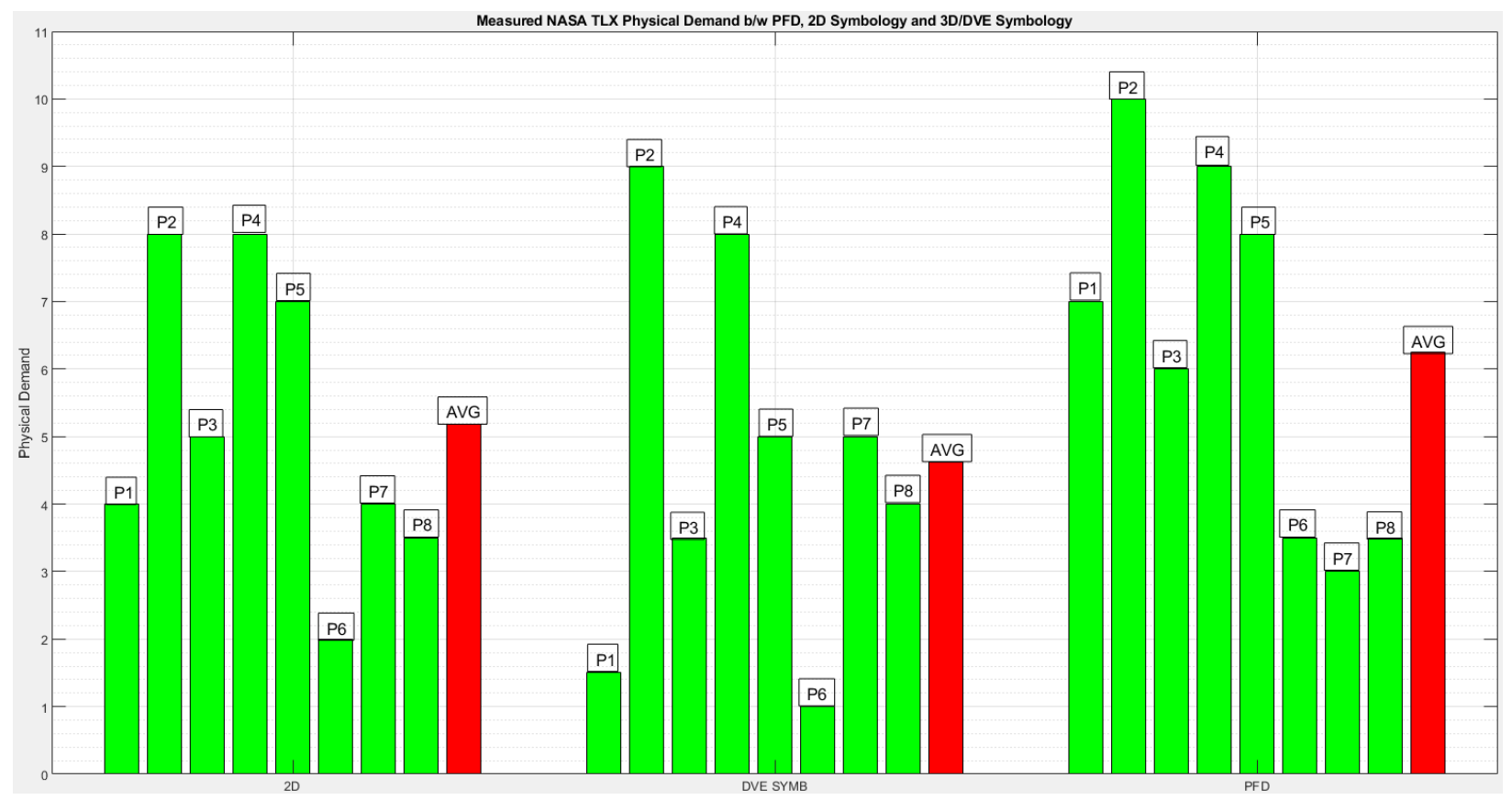

FIGURE 77: PHYSICAL DEMAND EXPERIENCED SURVEY RESULTS BY PARTICIPANTS USING PFD, 2D AND 3D DISPLAYS FROM NASA TLX PHYSICAL DEMAND SCALE.

The participants reported through the NASA TLX rating scale for Physical demand required that on average the physical demand level decreased when going from using the PFD display to the 2D orthographic display to the 3D DVE symbology respectively. As can be seen in Figure 77, in the PFD response, the average physical demand experienced was $62 \%$. The $2 \mathrm{D}$ orthographic response highlights the average effort value experienced was $52 \%$, showing a decrease in the required physical demand by $10 \%$. Similarly, using the 3D DVE symbology, the average effort decreased to $46 \%$, showing a significant decrease in the effort experienced by participants who were using the PFD display to fly and land. 


\section{Mental Demand}

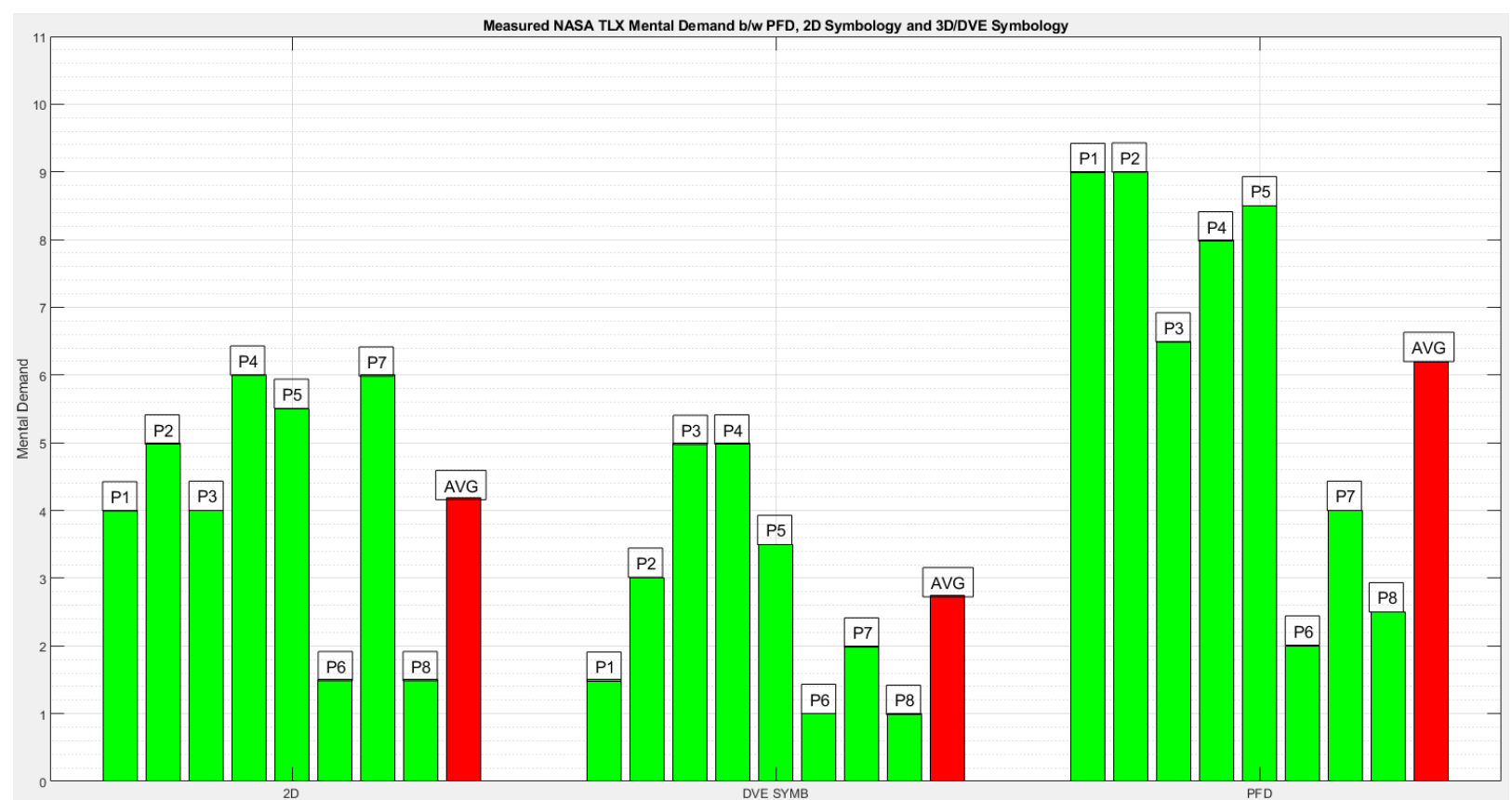

FIGURE 78: MENTAL DEMAND EXPERIENCED SURVEY RESULTS BY PARTICIPANTS USING PFD, 2D AND 3D DISPLAYS FROM NASA TLX MENTAL DEMAND SCALE.

The participants reported through the NASA TLX rating scale for mental demand required that on average, the mental demand level decreased when going from using the PFD display to the 2D orthographic display to the 3D DVE symbology. As can be seen in Figure 78, in the PFD response, the average mental demand experienced was $62 \%$. The $2 \mathrm{D}$ orthographic response highlights the average mental demand value experienced was $42 \%$, showing a decrease in the required effort by about $20 \%$. Similarly, using the 3D DVE symbology, the average mental demand decreased to $38 \%$, showing a significant decrease in the effort experienced by participants who were using the PFD display to fly and land. 


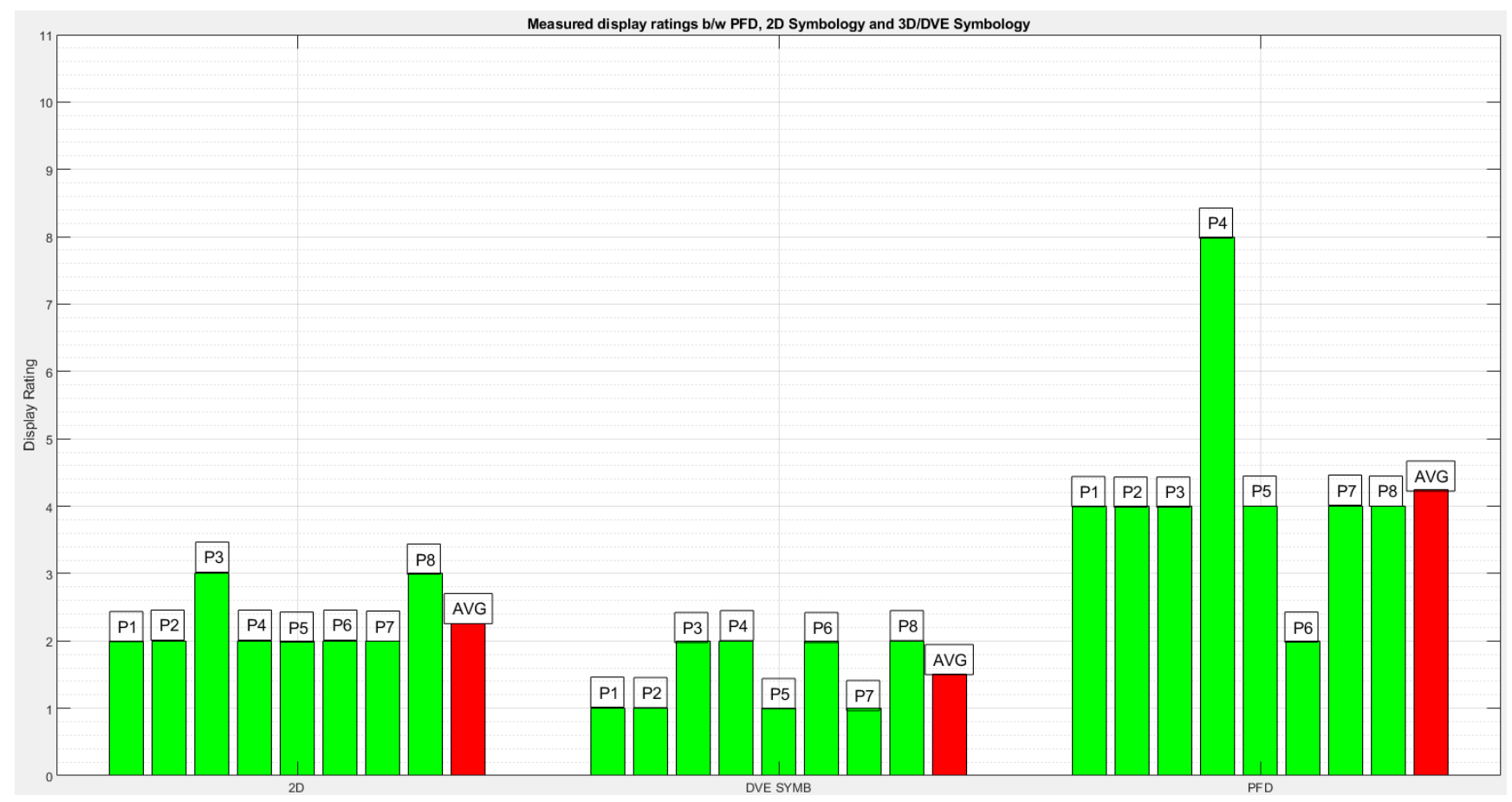

FIGURE 79: THE COOPER-HARPER SURVEY RESULTS BY PARTICIPANTS USING PFD, 2D AND 3D DISPLAYS.

Figure 79 shows the measured display rating shows the average rating for Cooper Harper scale decrease with the use of PFD, orthographic 2D symbology and 3D conformal DVE symbology. The average rating given for the PFD display was 4.3, which meant it provided for insufficient decision aiding. Insufficient decision making means the participant was not provided with multiple decision-making options, does not predict decision consequences nor convey information uncertainty. The $2 \mathrm{D}$ orthographic display ratings were about 2.3, which means the display provided right information with negligible deficiencies. Accurate and right information with negligible deficiencies means the display has minor negligible issues not hindering operating performance. Finally, the 3D conformal DVE symbology was given an average rating of 1.5, which means the display is highly desired. A highly desired display does not cause the operator to compensate for display irregularities. A 1.5 rating places the 3D DVE conformal symbology in the middle, between highly desired and good with negligible deficiencies. 


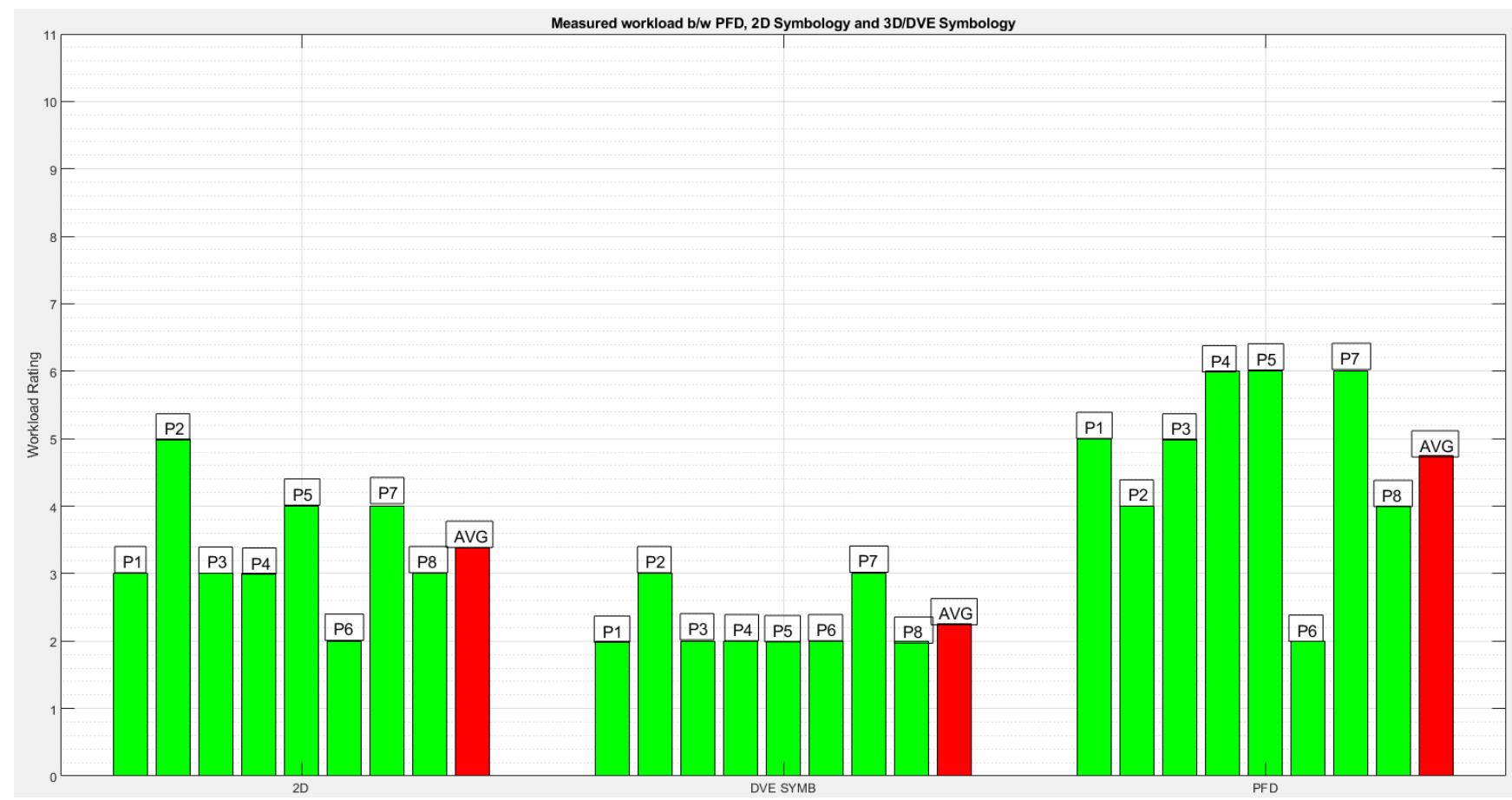

FIGURE 80: THE BEDFORD WORKLOAD SCALE SURVEY RESULTS BY PARTICIPANTS USING PFD, 2D AND 3D DISPLAYS.

Figure 80 shows the Bedford workload index scale was another non-intrusive questionnaire method used to analyze the workload experienced by the participants in using the three different displays (PFD, 2D Orthographic, and 3D conformal DVE). The ratings provided on average showed that using the PFD required higher workload to be maintained by the pilot. The PFD required, on average a 4.7 rating on the Bedford scale, which means there was insufficient or reduced spare capacity, with an inability to provide attention to additional tasks. The $2 \mathrm{D}$ orthographic display symbology on average had a Bedford workload rating of 3.4, which corresponds to enough spare mental capacity being available for desirable additional tasks. The 3D conformal symbology was given a Bedford workload rating index of 2.3 on average, which corresponds to low workload scenarios, with spare capacities for other additional tasks and actions. 


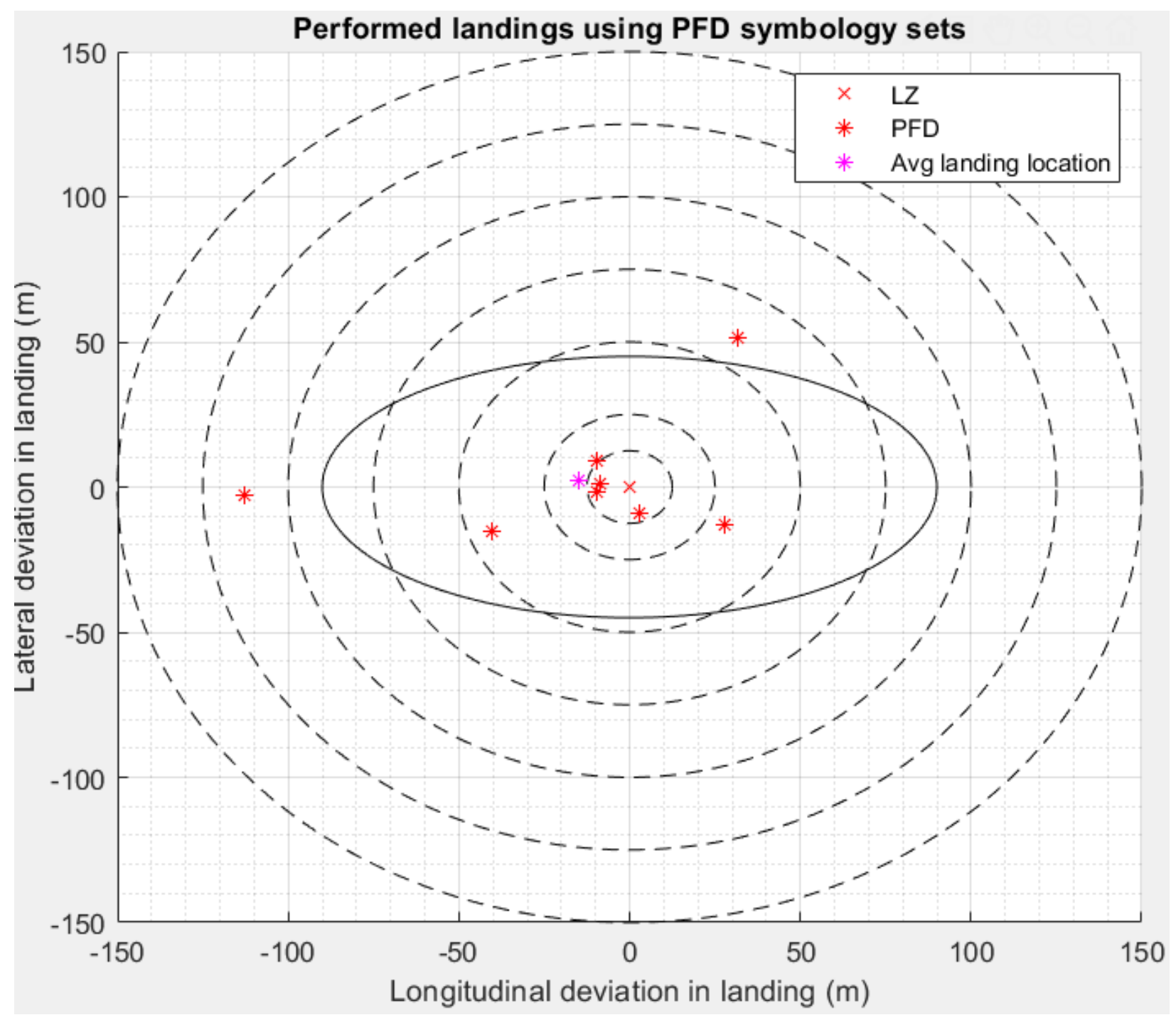

FIGURE 81: THE RECORDED LANDING POINTS USING THE PFD DISPLAY, WITH LATERAL AND LONGITUDINAL DEVIATION GRIDS. THE OVAL ABOUT THE CENTER REPRESENTS THE STADIUM.

Upon the completion of the flight test task, the participant was asked to land in the center of the football stadium. Using the PFD as cueing, all eight participants were able to land. Two participants landed outside the stadium, took off again, and landed inside the stadium again. By plotting the location of each participant's landing location, it was found the landings were further from the requested landing location. It was found three landings were made within a 12.5 -meter radius, one landing at 12.5 -meters from the requested location. Four landings were made outside a 25-meter radius with the furthest landing at a 112.5meter distance from the requested landing point. The average landing deviation was 14.8-meter from the center using the PFD. Figure 81 shows the locations of landings performed by the participants using the PFD display. 


\section{Landing Accuracy Results for 2D Symbology landings}

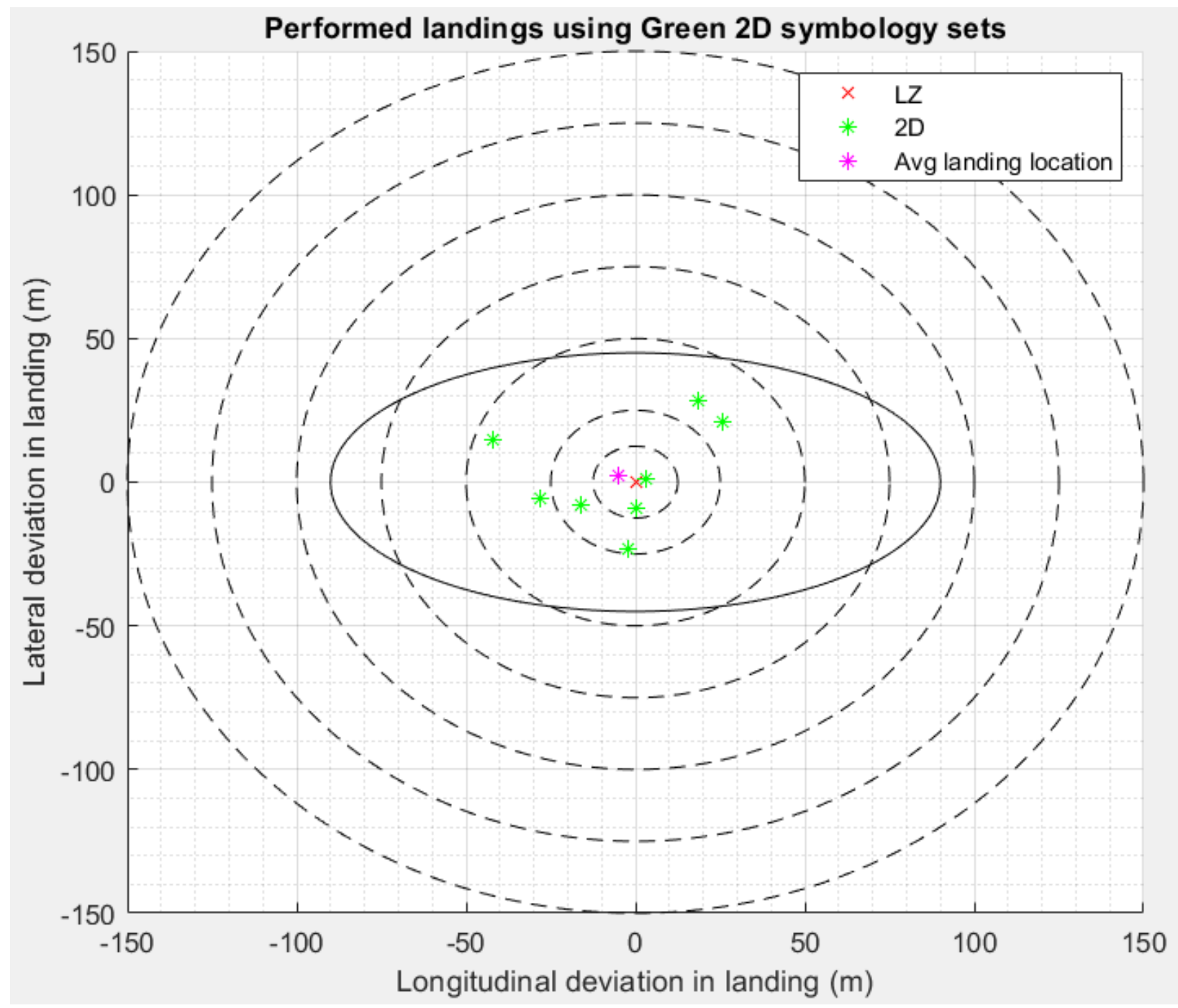

FIGURE 82: THE RECORDED LANDING POINTS USING THE 2D ORTHOGRAPHIC DISPLAY, WITH LATERAL AND LONGITUDINAL DEVIATION GRIDS. THE OVAL ABOUT THE CENTER REPRESENTS THE STADIUM.

Like the PFD test case, the participants were asked to perform the same landing using the 2D orthographic symbology. Using the 2D orthographic symbology as cueing, all eight participants were able to land. All the participants were able to land inside the stadium. By plotting the location of each participant's landing location, it was found the landings were grouped closer with the 2D orthographic symbology. It was found that two landings were made within a 12.5-meter radius; two landings were made between 12.5-meters and 25 -meters radiuses from the requested location. Four landings were made outside a 25 -meter radius with the furthest landing at a 42-meter distance from the requested landing point. Though there were fewer landings within the 12.5-meter radius circle, the landings were closer to the center without large deviations. The average landing deviation was 5.6-meter from the center using the 2D orthographic symbology. Figure 82 shows the locations of landings performed by the participants using the $2 \mathrm{D}$ display. 


\section{Landing Accuracy Results for 3D Symbology landings}

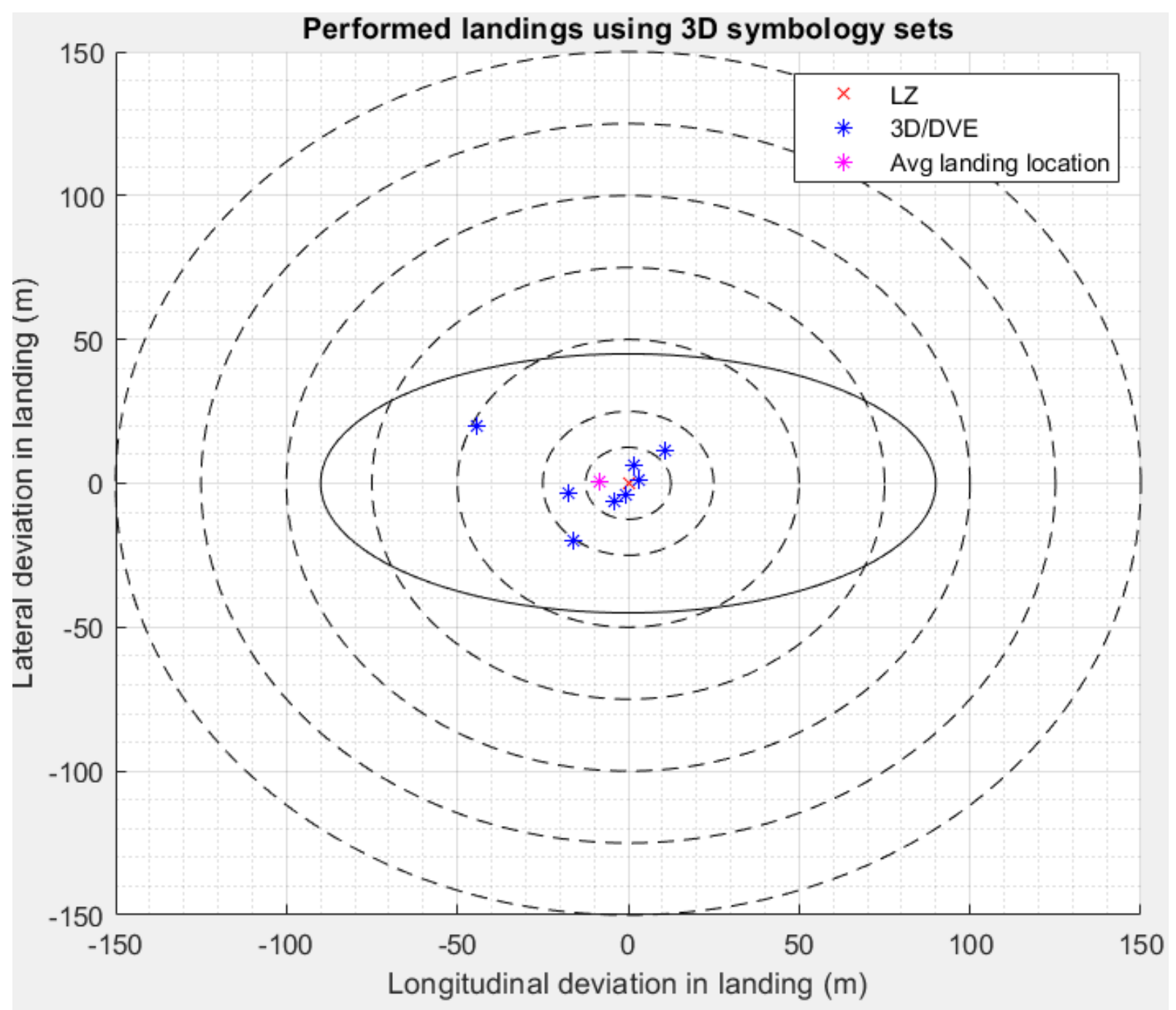

FIGURE 83: THE RECORDED LANDING POINTS USING THE 3D CONFORMAL DISPLAY, WITH LATERAL AND LONGITUDINAL DEVIATION GRIDS. THE OVAL ABOUT THE CENTER REPRESENTS THE STADIUM.

Just as with the PFD and 2D orthographic test case, the participants were asked to perform the same landings using the 3D conformal symbology. Using the 3D conformal symbology as cueing, all eight participants were able to land inside the stadium. By plotting the location of each participant's landing location, it was found the landings were grouped closer with the 3D conformal symbology. It was found that four landings were made within a 12.5-meter radius. Three landings were made between 12.5-meters and 25 -meters radiuses from the requested location. One landing was made outside a 25 -meter radius with the furthest landing at a 50-meter distance from the requested landing point. The landings were closer to the center without large deviations. The average landing deviation was 8.4-meter from the center using the 3D conformal symbology. Figure 83 shows the locations of landings performed by the participants using the $3 \mathrm{D}$ and $2 \mathrm{D}$ display. 


\section{Texture Test Results}

The flight test from both the participants was performed on three different textures, the mesh-based hexagonal, mesh-based square, and the checkerboard. The rating was based on its ability to help perceive information from the texture. The perception of altitude and speed were asked to be maintained. The participant was asked to maintain a certain altitude and speed (the X-axis in Figures 84-86) by flying utilizing the texture as cueing for altitude and speed. Altitudes of only up to 50 feet were used as the testing criteria, as this is the general altitude at which brownout/white-outs start forming.

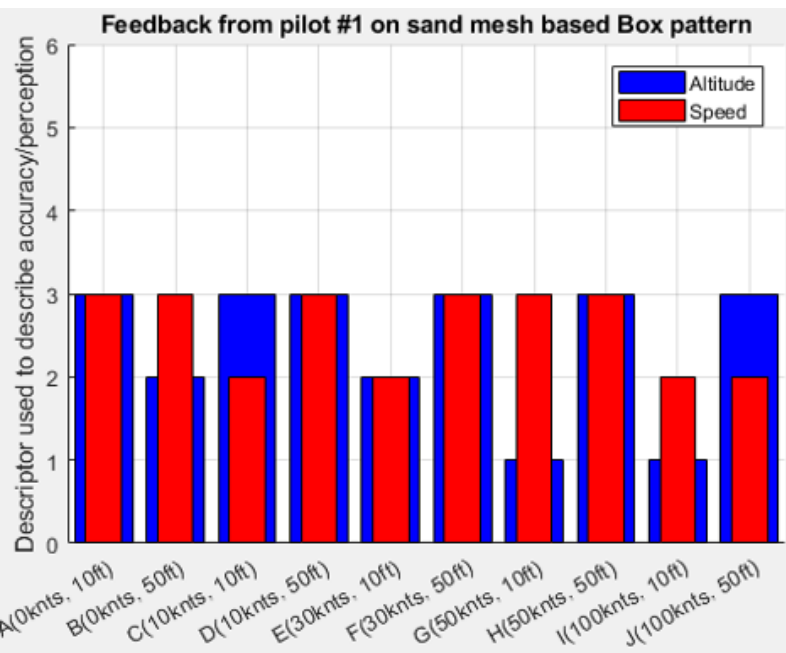

Speed and Altitude combinations

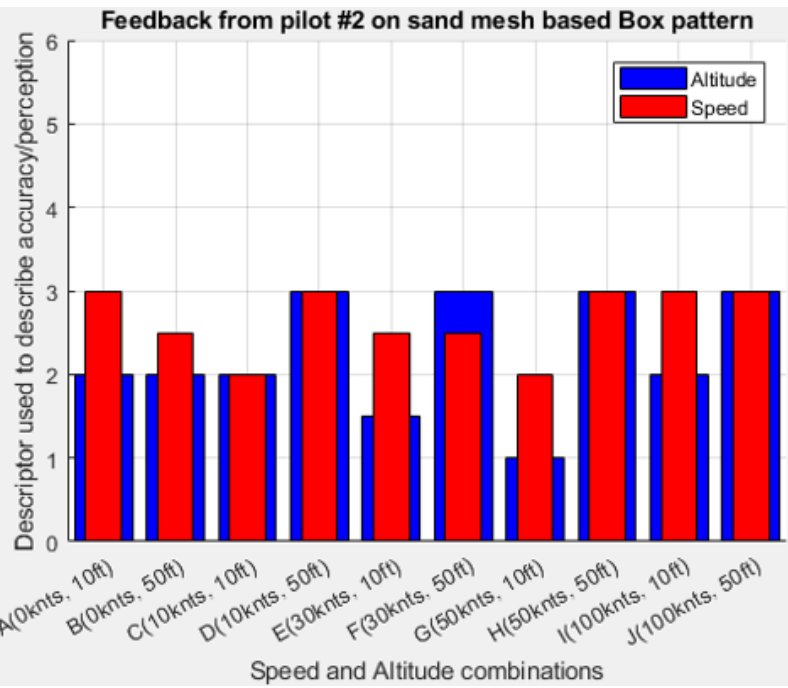

Speed and Altitude combinations

FIGURE 84: FEEDBACK FROM PARTICIPANTS 1 AND 2 FOR THE SANDMESH-BOX TEXTURE.

The results of the first flight test, which used the sand mesh-based box pattern showed that among both participants, the altitude perception was rated bad to poor at low altitudes and low speeds. The perception was rated fair to good at higher speeds and higher altitudes. The higher speeds/altitudes in statements are at about 50 feet and greater than 50 knots. The lower speeds and altitudes stated are speeds less than or equal to 30 knots and less than 50 feet. 

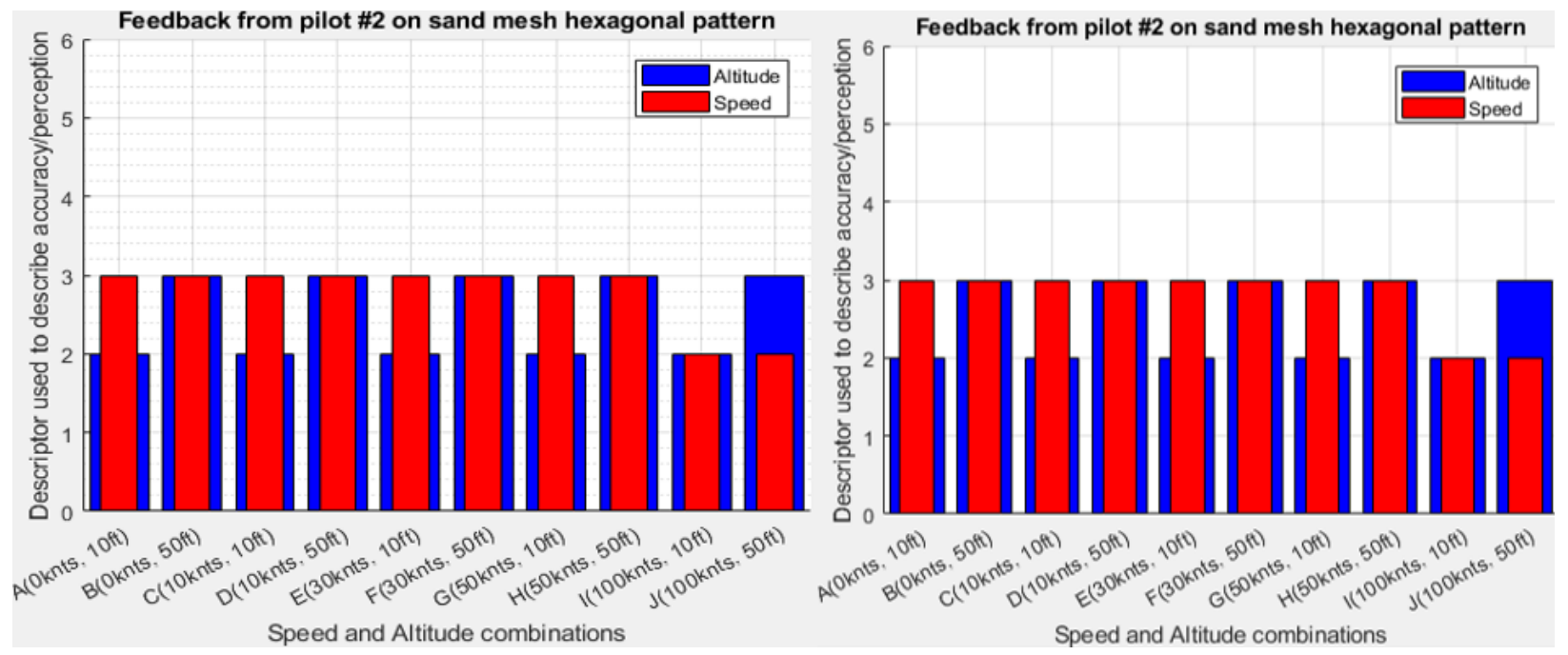

FIGURE 85: FEEDBACK FROM PARTICIPANTS 1 AND 2 FOR THE SANDMESH-HEX TEXTURE.

The results of the first flight test which used the sand mesh-based hexagonal pattern showed that among both participants the altitude perception was rated mostly good by participant one at both low altitude, low speed and higher altitude and higher speed conditions. The second participant echoed a similar response but rating the texture as fair in higher speed conditions at both low and higher speed conditions. The higher speeds/altitudes in statements are at about 50 feet and greater than 50 knots. The lower speeds and altitudes stated are speeds less than or equal to 30 knots and less than 50 feet.
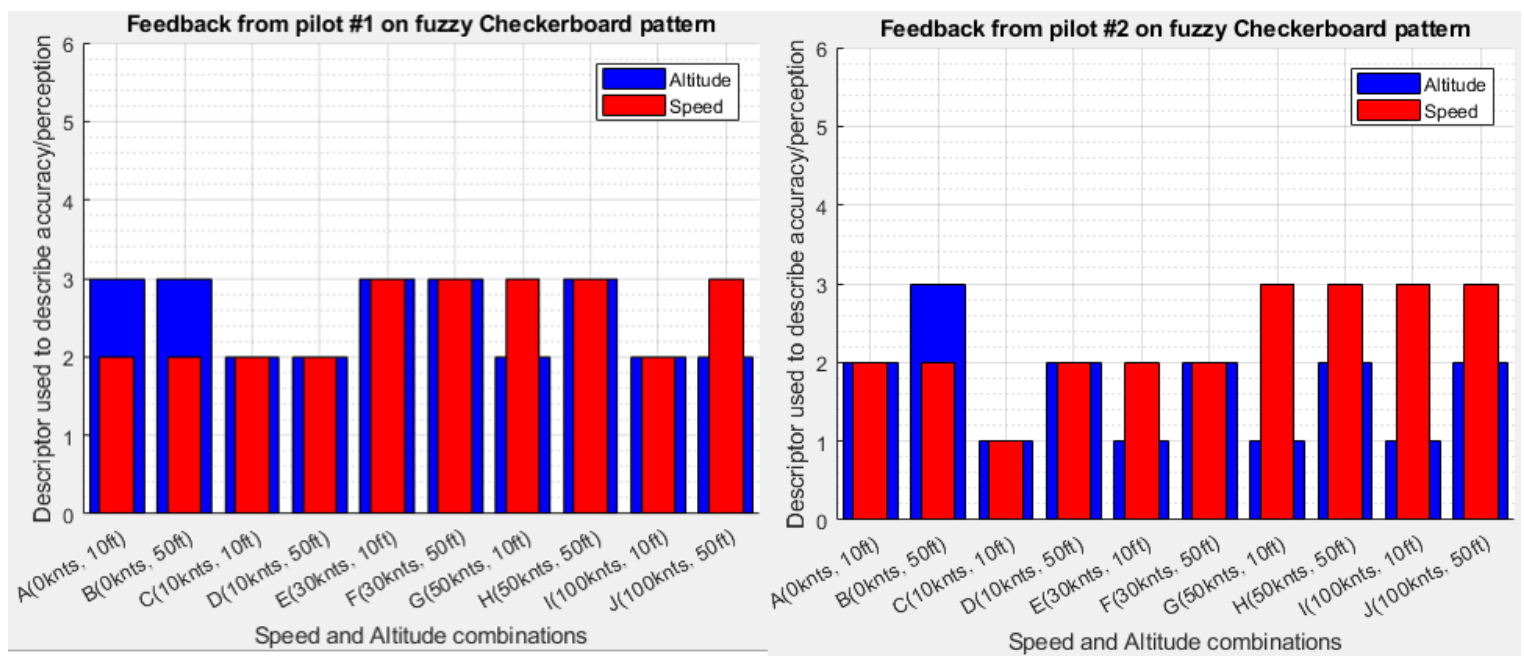

FIGURE 86: FEEDBACK FROM PARTICIPANTS 1 AND 2 FOR THE CHECKERBOARD PATTERN TEXTURE.

The results of the first flight test, which used the checkerboard pattern showed that among both participants, the perception was rated fair to good at higher speeds and lower altitudes. The perception was rated poor at low altitudes and lower speeds. The higher speeds/altitudes in statements are at about 50 feet and greater than 50 knots. The lower speeds and altitudes stated are speeds less than or equal to 30 knots and less than 50 feet. 


\subsection{SUMMARY AND DISCUSSION}

The results from the flight test can be summarized through Table 8 and figure 87 .

TABLE 8: SUMMARY OF RESULTS FROM THE NON-INTRUSIVE QUESTIONNAIRE RESULTS.

$\begin{array}{cccc}\text { Measurement/Symbology } & \text { PFD (Avg) } & \begin{array}{c}\text { Orthographic Symbology } \\ (\text { Avg })\end{array} & \begin{array}{c}\text { 3D Conformal Symbology \& } \\ \text { Orthographic (Avg) }\end{array} \\ \text { NASA TLX: Mental } & 6.2 & 4.2 & 3.8 \\ \text { NASA TLX: Physical } & 6.2 & 5.2 & 4.6 \\ \text { NASA TLX: Temporal } & 5.9 & 2.9 & 3.6 \\ \text { NASA TLX: Effort } & 6.5 & 4.7 & 3.8 \\ \text { NASA TLX: Frustration } & 6.4 & 4.3 & 2.7 \\ \text { NASA TLX: Performance (Self) } & 4.6 & 3.0 & 1.9 \\ \text { Workload (Bedford) } & 4.7 & 3.4 & 2.3 \\ \text { Cooper-Harper Display } & 4.3 & 2.3 & 1.5 \\ \text { Ratings } & & & \end{array}$

Through flight testing and non-intrusive questionnaires, it was found the mental, physical, effort, frustration demand experienced by the participants decreased as they went using the PFD display to 2D orthographic display to the $3 \mathrm{D}$ conformal symbology display as a cue to perform the requested flight operations and landings. It was found the temporal demand decreased between using the PFD cueing display and the 2D orthographic symbology, however, increasing again using the 3D conformal symbology. 


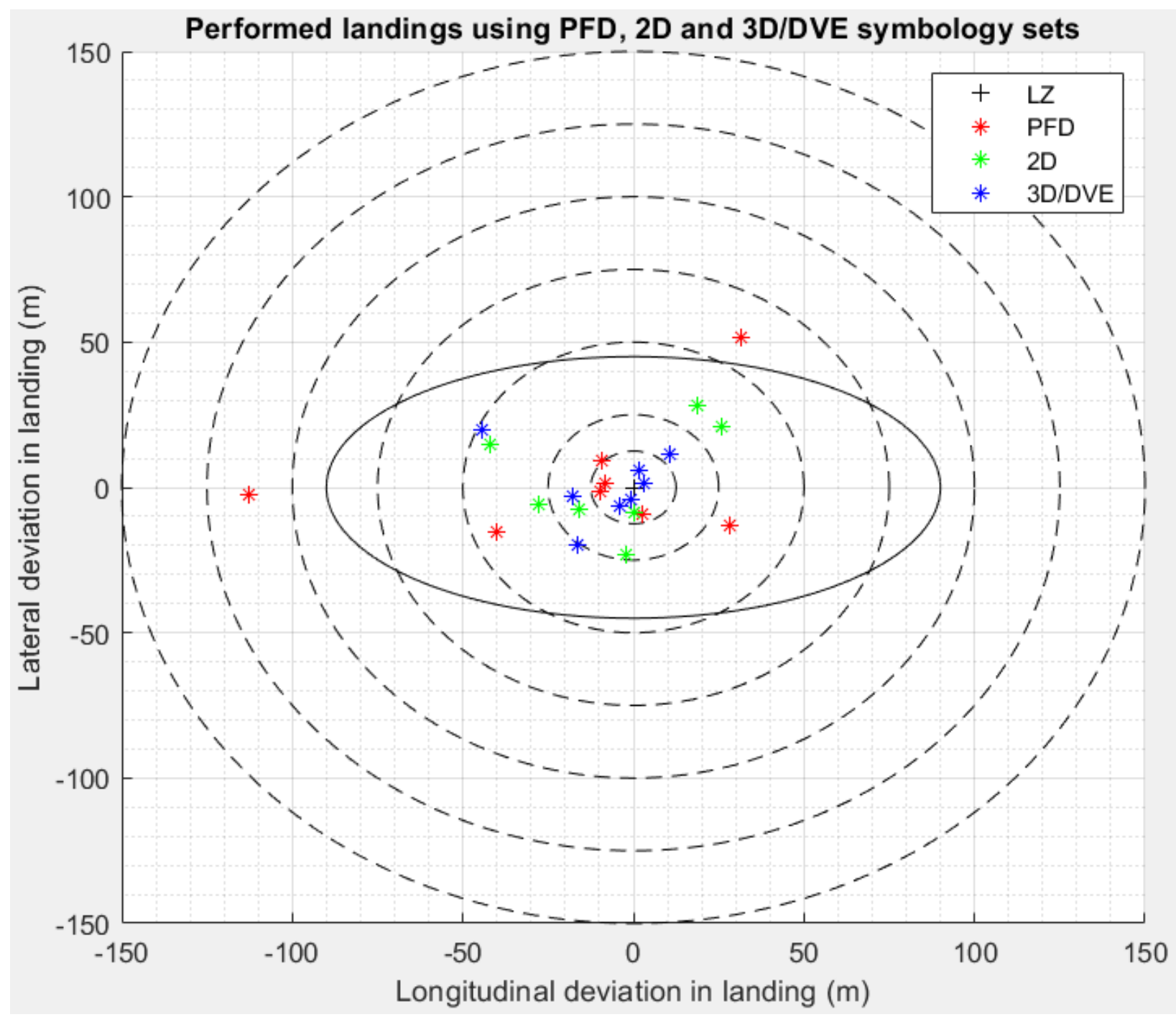

FIGURE 87: THE RECORDED LANDING POINTS USING THE PFD, 2D ORTHOGRAPHIC AND 3D CONFORMAL DISPLAYS, WITH LATERAL AND LONGITUDINAL DEVIATION GRIDS. THE OVAL ABOUT THE CENTER REPRESENTS THE STADIUM.

The accuracy of the landings was found to be grouped closer together with the use of 2D orthographic symbology over the PFD display and were progressively better using both the 2D orthographic and 3D conformal displays overlaid together. It was found the average deviation of the landings were 15-meter using the PFD, 5.6-meter using the 2D orthographic and 8.5-meter using the 3D conformal symbology. The illustration of the landings using the three display sets can be seen in Figure 87. 


\section{CHAPTER 6.0 CONCLUSION}

The objective of the study was to research and develop a cueing system which helps helicopter pilots operate and land in DVE in low altitude operations. A comprehensive literature survey was performed highlighting the critical areas of interest and methods of cueing which exist to help pilots in DVE. Through a literature survey, existing standards on the information required by pilots operating in low visibility environments was understood. The literature survey established sources of information which are required by pilots and are missing in the case of advertent or inadvertent entry into DVE. A lack of information that is required by the pilot to land a helicopter can cause a lapse in the information processing and decision-making process of the pilot. Lapses lead to decision mistakes and can cause accidents if left unmitigated. The Taxonomic framework for assessing aircrew error described in chapter 2.2, helps to provide an iterative approach to fill areas in which there is a lack of information to make appropriate decisions and mitigate issues before they arise.

It was found that in a DVE situation, pilots do not have access to information on the lateral, longitudinal and vertical drift, ground slope, terrain features, landing point location, obstacle clearance, and landing direction. Landing direction establishment is performed as part of high and low altitude reconnaissance; however, precise orientation is required when performing a steep or low and rapid descent approaches. Through the established lack of information, the O'Hare et al. "A taxonomic framework for assessing aircrew error" was used to provide the information missing due to a DVE. The introduced information was in the form of 2D orthographic symbology and 3D conformal symbology represented via a visual HDD. A terrain texture pattern was created to provide the pilot with drift cues in low altitude hover and also provide terrain flow information by conforming to the DEM data. The display effectively provided the pilot with the helicopter information (2D symbology) and information about the terrain and environment (3D symbology) building a mental picture and information to operate effectively in DVE in low-altitude operations.

After the development of the display symbology, it was tested through volunteer participants to evaluate the usability, workload, and stress in using the display of the display, terrain textures and to measure the improvement in workloads, stress and operator performance through recorded flight test data and nonintrusive questionnaires. The Bedford workload index, NASA TLX rating scale, and the modified CooperHarper display rating scale were all used to test the improvement in performance, decrease in stress and workloads. The testing was conducted at the Ryerson MIMS lab's Fixed Base Simulator, imitating DVE conditions. The participants were asked to take off at an airfield and maintain hover and perform a landing inside a stadium. The participants were asked to perform the flight test three times, using a generic PFD display (used in most modern helicopter flight decks), using the 2D orthographic symbology and finally 
with the 3D conformal symbology. The participant's flight was recorded, and their responses for questionnaires were recorded as well. As reported in chapter 5.3, the mental, physical, effort, frustration demand experienced by the participants decreased using the PFD, 2D, and 3D symbology. The participants reported their rating of self-performance to have increased between the PFD, 2D orthographic symbology and the 3D conformal symbology. It was found that the temporal demand decreased between using the PFD cueing display and the 2D orthographic symbology. However, increasing again using the 3D conformal symbology from 2D orthographic symbology. According to participant comments, the increase in temporal demand was due to them seeing the destination and feeling an urgency to land in the requested landing zone.

The accuracy of the landings was also determined, it was found to be grouped closer together with the use of $2 \mathrm{D}$ orthographic symbology over the PFD display and were progressively better using both the $2 \mathrm{D}$ orthographic and 3D conformal displays overlaid together. There were some deviations even with the 3D and 2D overlay display; this can be amounted to the in-experience of the participants, as they all had limited experience to helicopter flight and performing precise landings. The comments from the participants also gave insight into what the display provide or fail to provide to improve operation.

Overall, the development process and its complementary flight tests phase show that there is a definite improvement in the performance of pilots. Survey of the participants and participant comments highlighted that the PFD display was poor for delicate tasks such as hover and worse for situations where there were no VFR cues. The participants reported that the $2 \mathrm{D}$ orthographic symbology was extremely useful with only pertinent information being displayed, and the acceleration and velocity cueing were extremely useful in maintaining and performing critical maneuvers. It was widely reported that the 3D conformal symbology served its purpose of providing a Virtual-VFR representation and helping the pilots navigate and operate effectively. The overall performance of participants has improved, and the system proved to be very beneficial for application in DVE for helicopter operations in low altitude in cases of hover and low altitude maneuvering.

\subsection{FUTURE WORKS}

The system developed as is not perfect, like any other system. The DVENS project capabilities and especially the symbology helped the pilot decrease workload and stress and help perform landings more effectively in DVE. In future iterations of the display, the two hover and cruise phases would be combined to such that the CAM would act to show pitch information to the pilot. This would limit the switching between the symbology and maintain uniformity. Another planned development is the integration of dedicated 3D waypoint symbology. A capability which will allow a pilot to enter waypoints and these will be displayed via the 3D waypoint symbology. A shortcoming the project has the representation of obstacles effectively, static and dynamic obstacles were represented as wireframe cubes 
to inform the pilot of an imminent obstacles. The system however did not allow for the classification of the obstacles nor did it effectively show or help the pilot understand what the obstacles is. Thus, as part of the future works a system which allows the LiDAR or capable camera system to signify what the obstacle is would be a sizeable achievement for pilot understanding of scenario. Using a camera to identify and classify an object is known as Computer Vision Methods (CVM) and has seen popular use in Robotics and the autonomous Automotive industry. As a trend towards automation increases, a new automated path planning initiative would be introduced with the respective virtual symbology representation. The path planning concept would allow the pilot to enter a destination location, and an automatic path would be generated considering on available information of weather, hostile-zones, restricted areas. The combination of CVM and path planning would benefit pilot understanding while decreasing workload and stress. The path planning and CVM initiative allows for future orientation of autonomous flight for Remotely Piloted Aircraft Systems (RPAS). 


\section{APPENDICES}

\section{APPENDIX A: GRAPHS FOR HOVER PHASE}

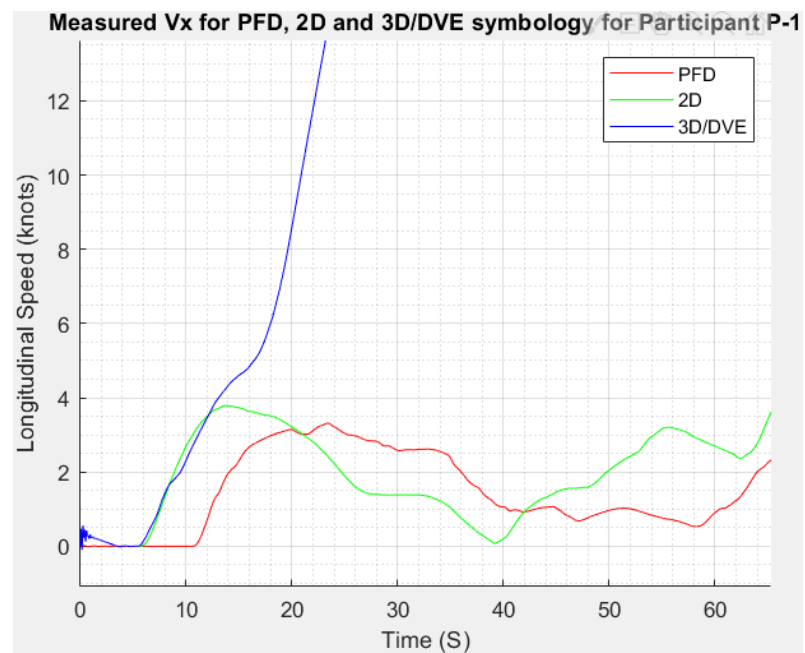

FIGURE A-1: MEASURED LONGITUDINAL SPEED FOR PARTICIPANT 1 IN HOVER.

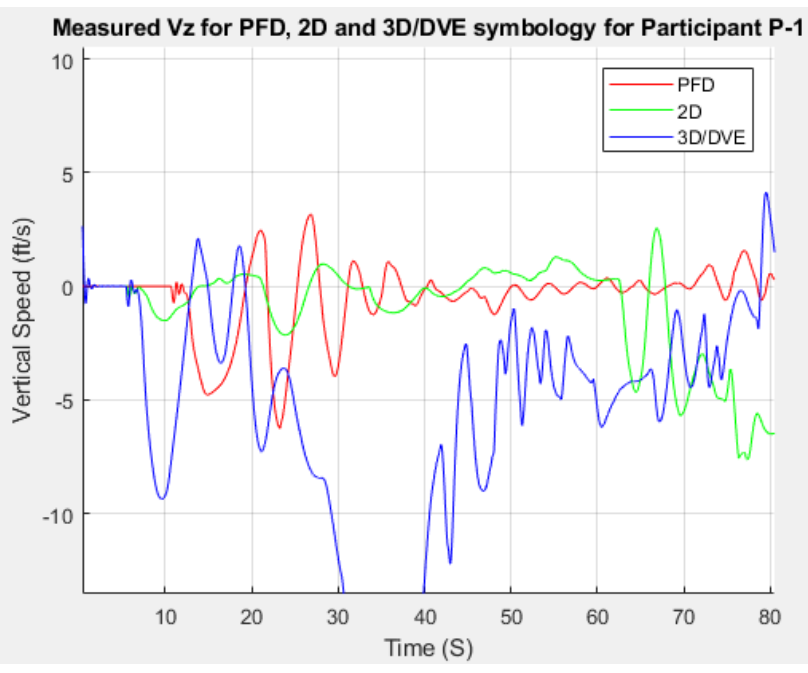

Figure A-2: MEASUREd VERTICAL VELOCITY FOR PARTICIPANT 1 IN HOVER.

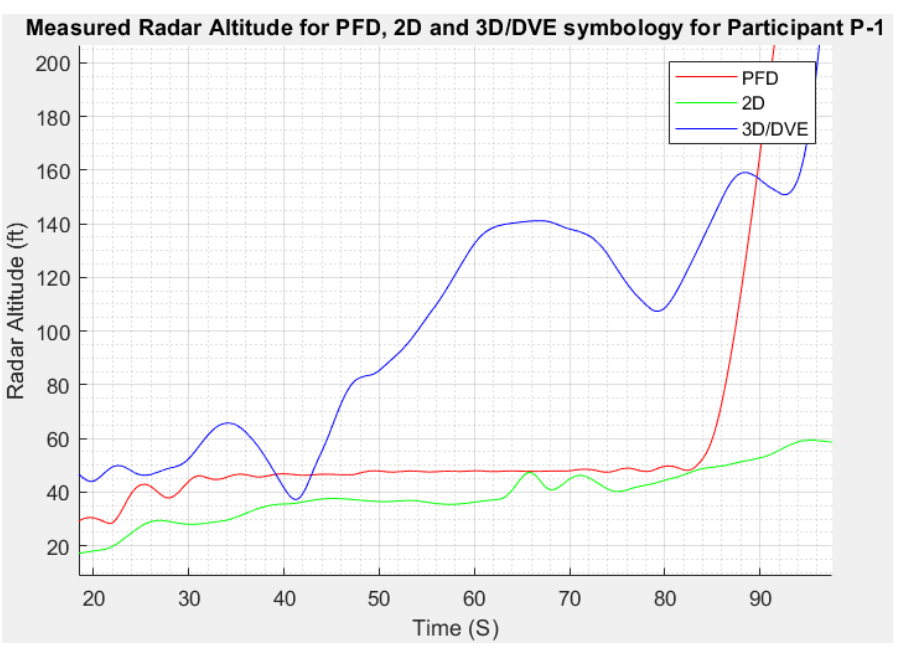

FIGURE A-3: MEASURED RADAR ALTITUDE FOR PARTICIPANT 1 IN HOVER.

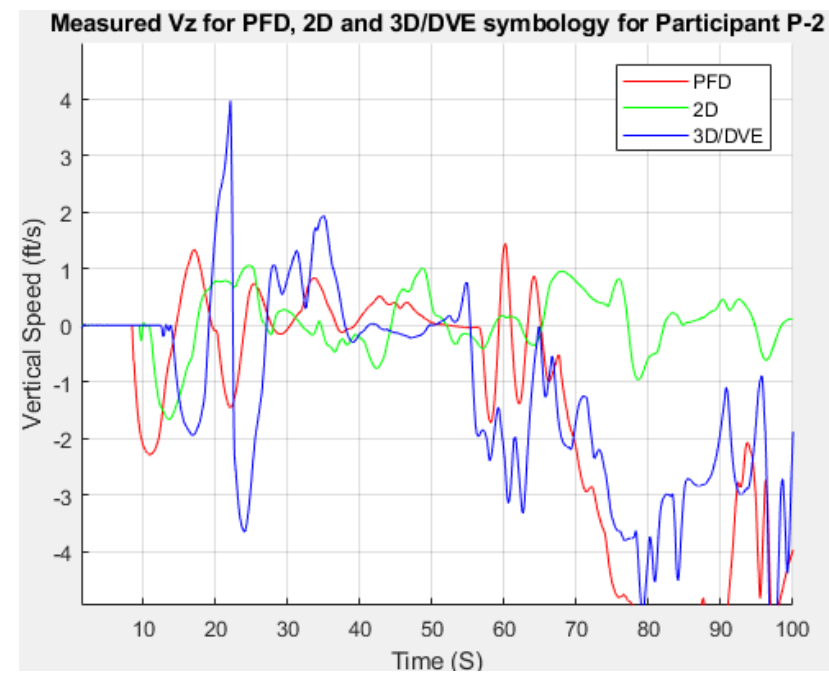

Figure A-4: MEASUREd VERTICAL VELOCITY FOR PARTICIPANT 2 IN HOVER. 


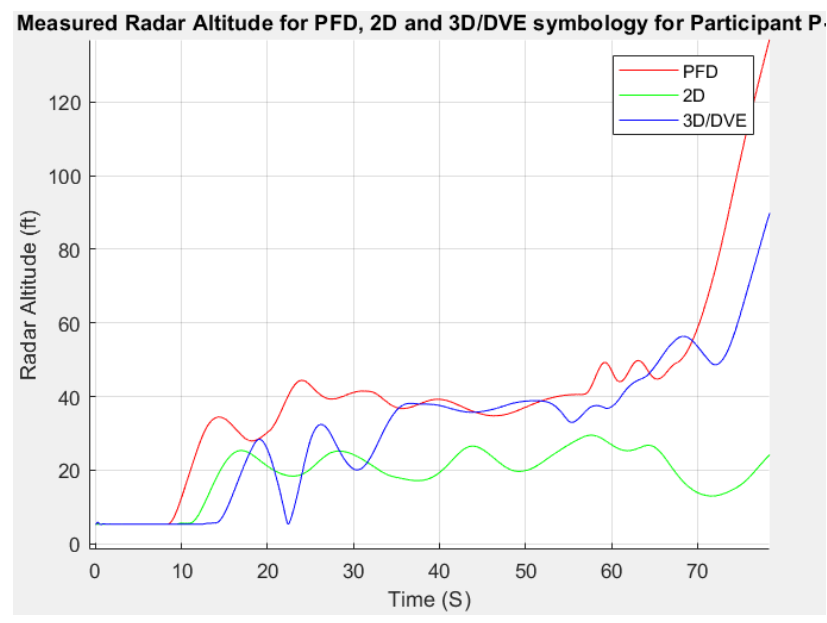

FIGURE A-5: MEASURED RADAR ALTITUDE FOR

PARTICIPANT 2 IN HOVER.

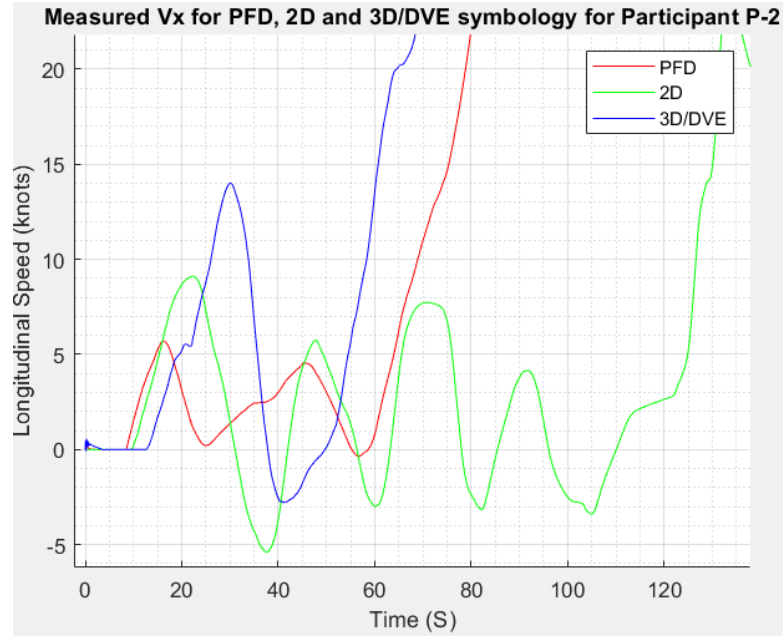

FIGURE A-6: MEASURED LONGITUDINAL VELOCITY FOR PARTICIPANT 2 IN HOVER.

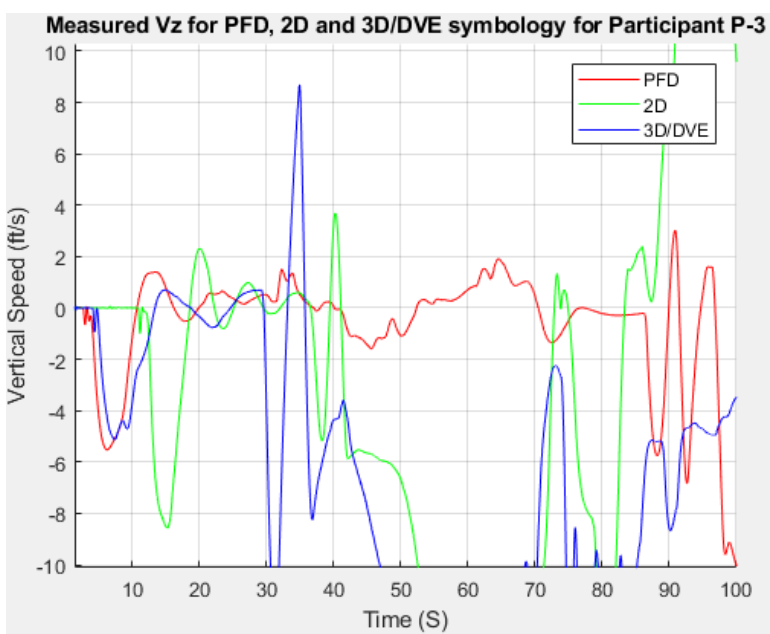

FigURE A-7: MEASURED VERTICAL VELOCITY FOR PARTICIPANT 3 IN HOVER.

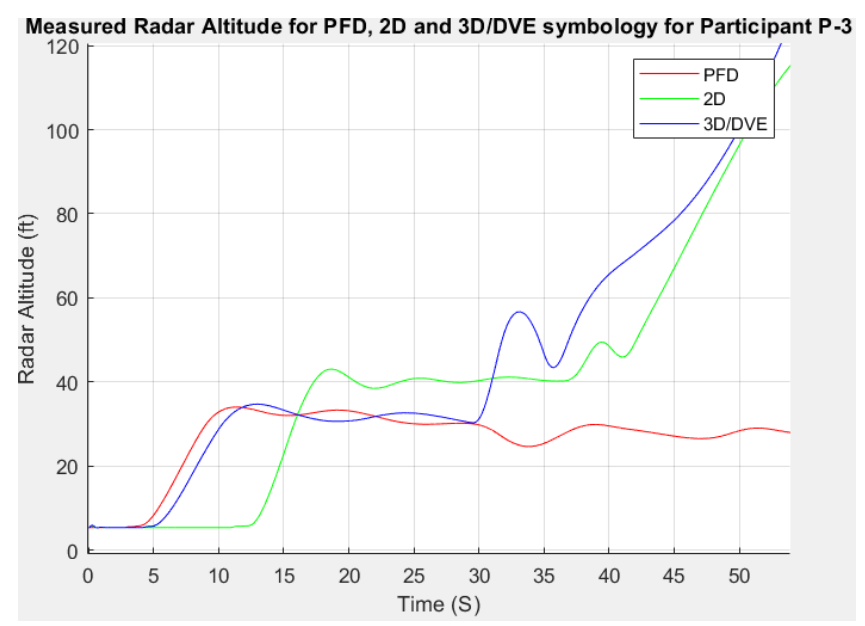

FIGURE A-8: MEASURED RADAR ALTITUDE FOR PARTICIPANT 3 IN HOVER. 


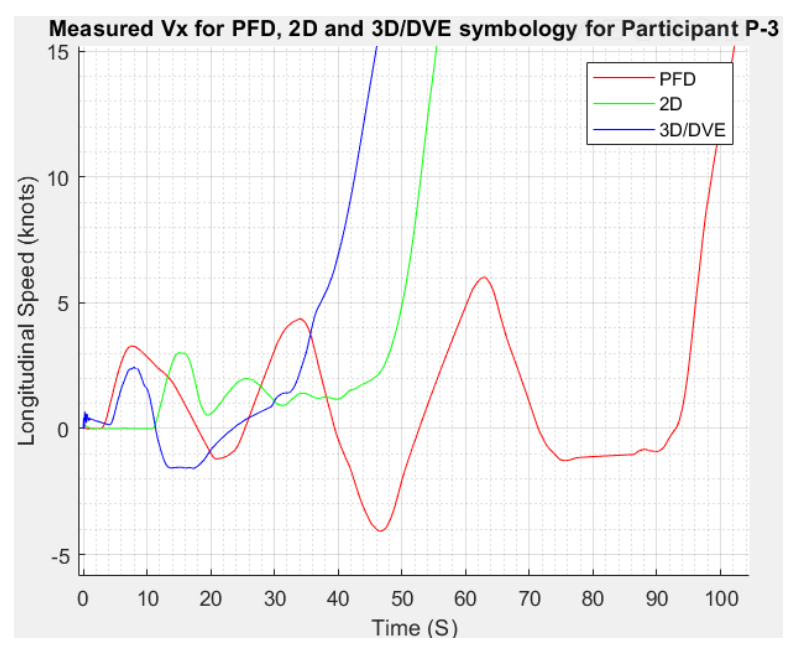

Figure A-9: MEASURED LONGITUDINAL VELOCITY FOR PARTICIPANT 3 IN HOVER.

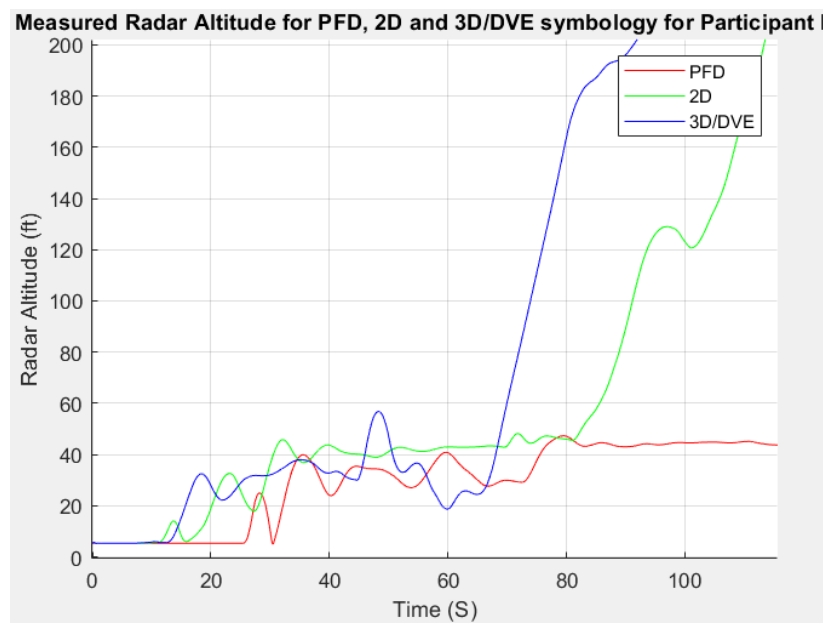

FIGURE A-10: MEASURED RADAR ALTITUDE FOR PARTICIPANT 3 IN HOVER.

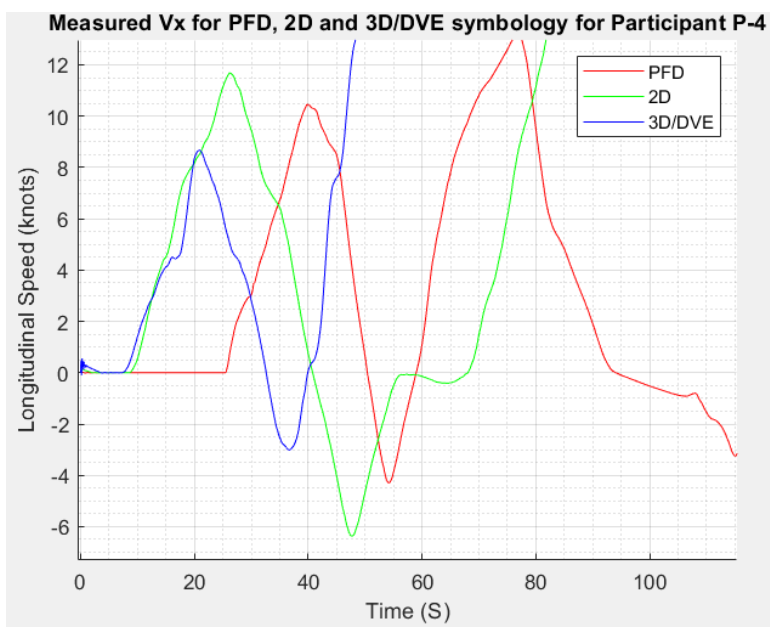

FIGURE 88:MEASURED LONGITUDINAL SPEED FOR PARTICIPANT 4 IN HOVER.

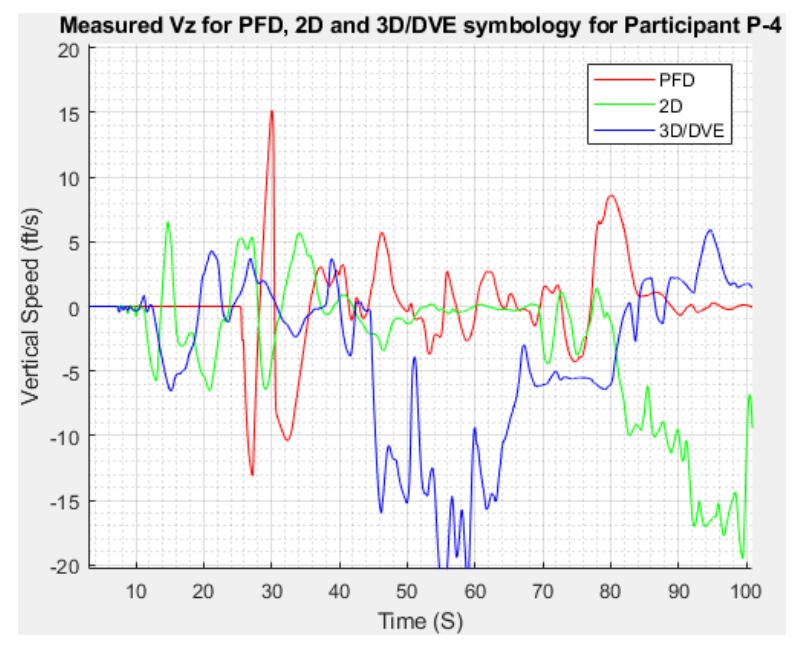

FIGURE A-11: MEASURED VERTICAL SPEED FOR PARTICIPANT 4 IN HOVER. 


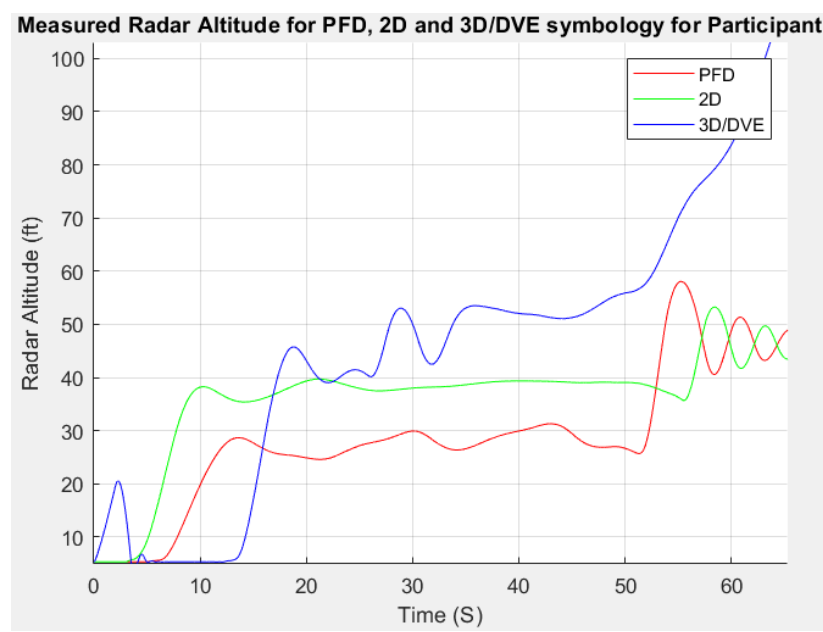

FIGURE A-12: MEASURED RADAR ALTITUDE FOR PARTICIPANT 5 IN HOVER.

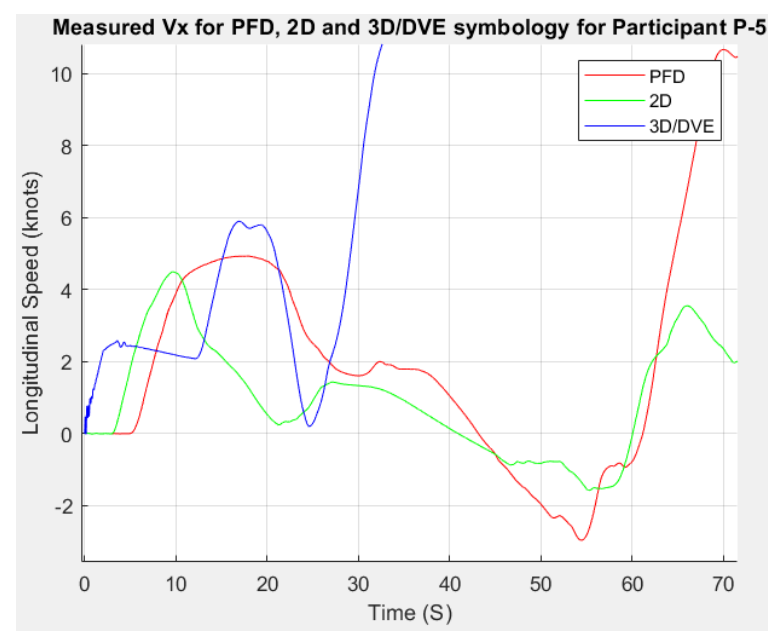

FIGURE A-13: MEASURED LONGITUDINAL SPEED FOR PARTICIPANT 5 IN HOVER.

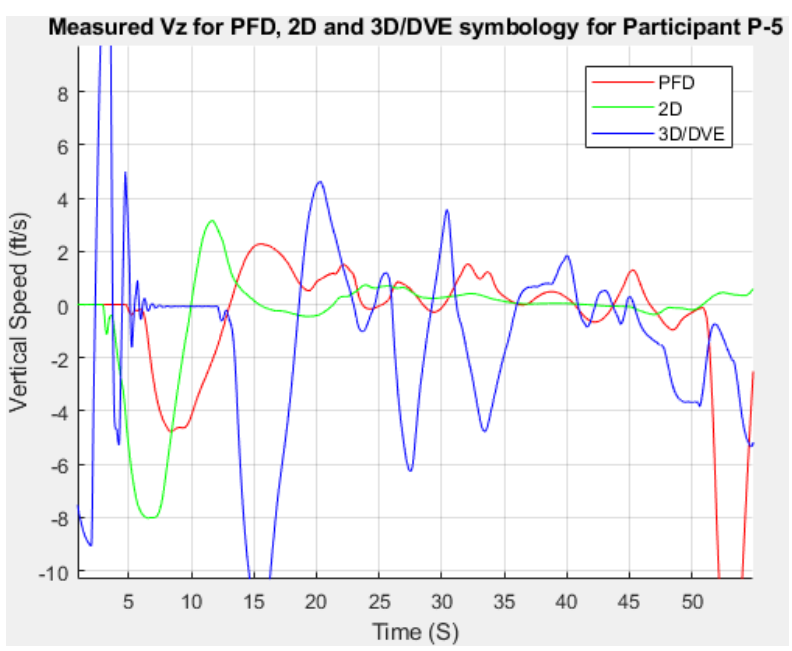

Figure A-14: MEASURED VERTICAL VELOCITY FOR PARTICIPANT 5 IN HOVER.

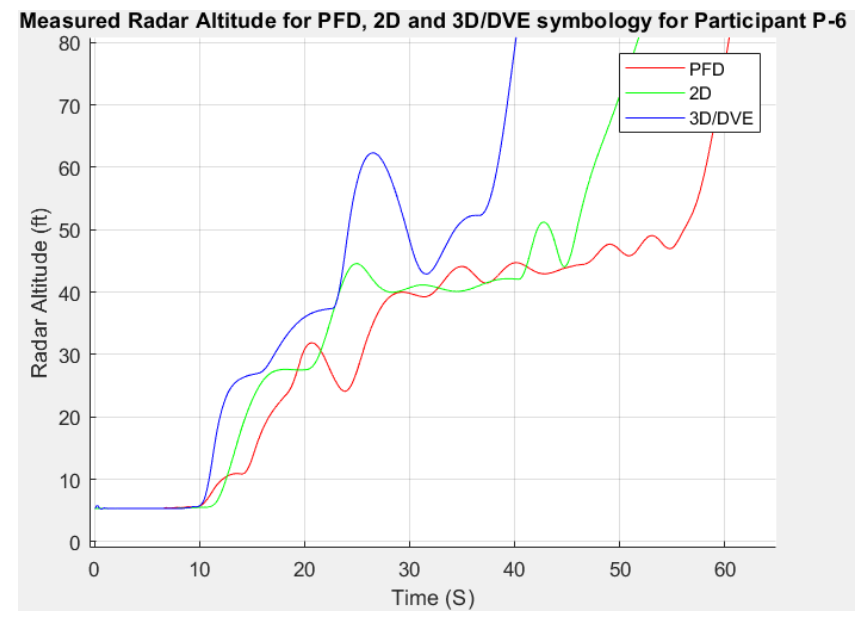

FIGURE A-15: MEASURED RADAR ALTITUDE FOR PARTICIPANT 6 IN HOVER. 


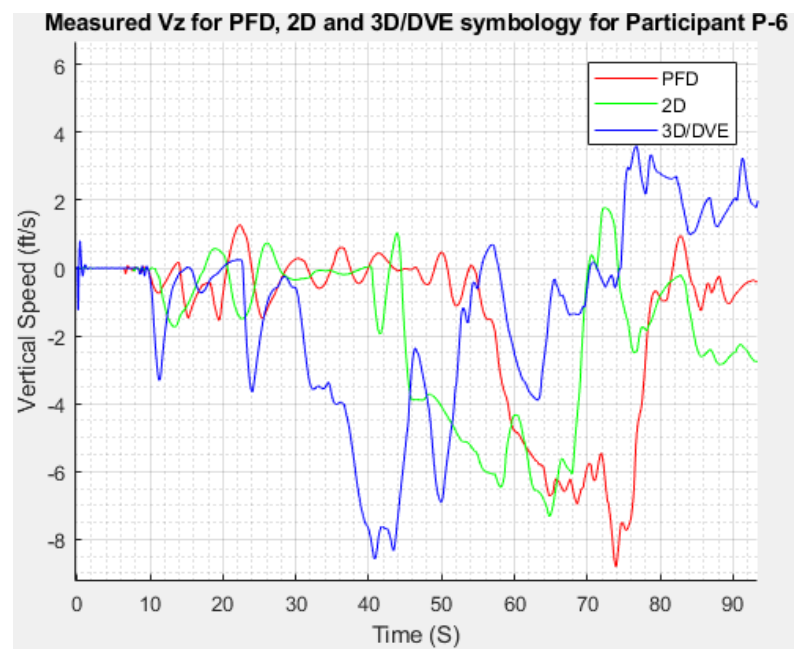

Figure A-16: MEAsured Vertical Velocity for PARTICIPANT 6 IN HOVER.

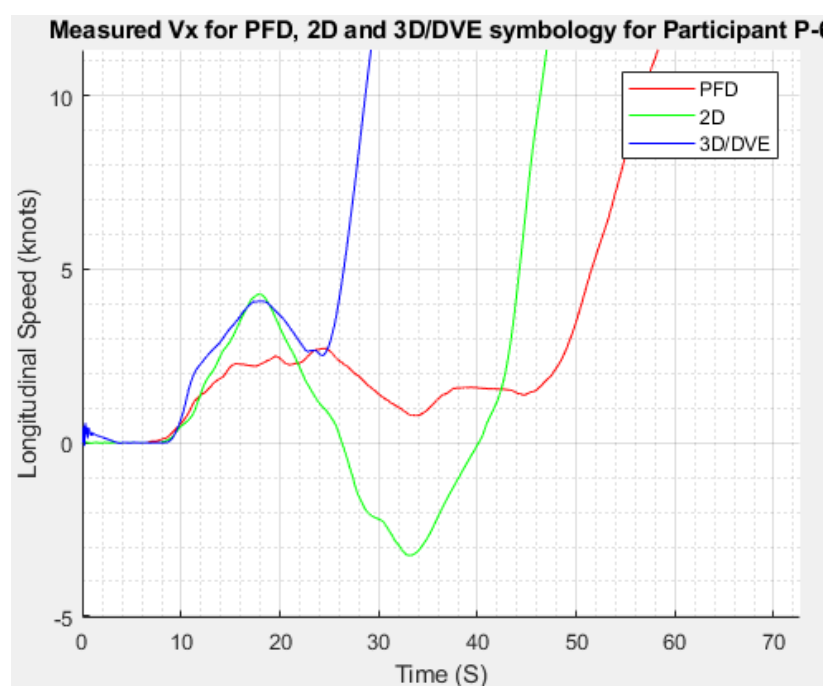

FigURE A-17: MEASURED LONGITUdINAL SPEED FOR

PARTICIPANT 6 IN HOVER.

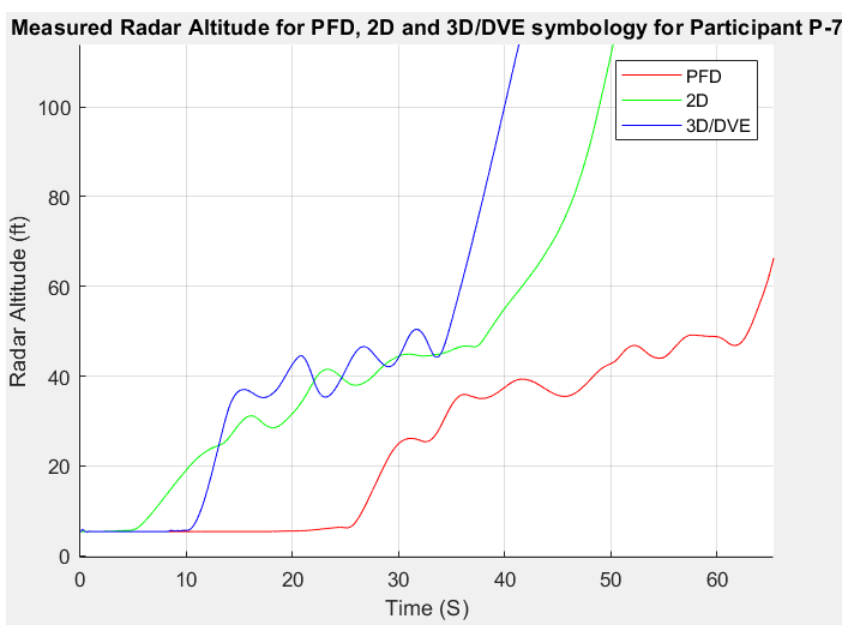

FIGURE A-18: MEASURED RADAR ALTITUDE FOR PARTICIPANT 7 IN HOVER.

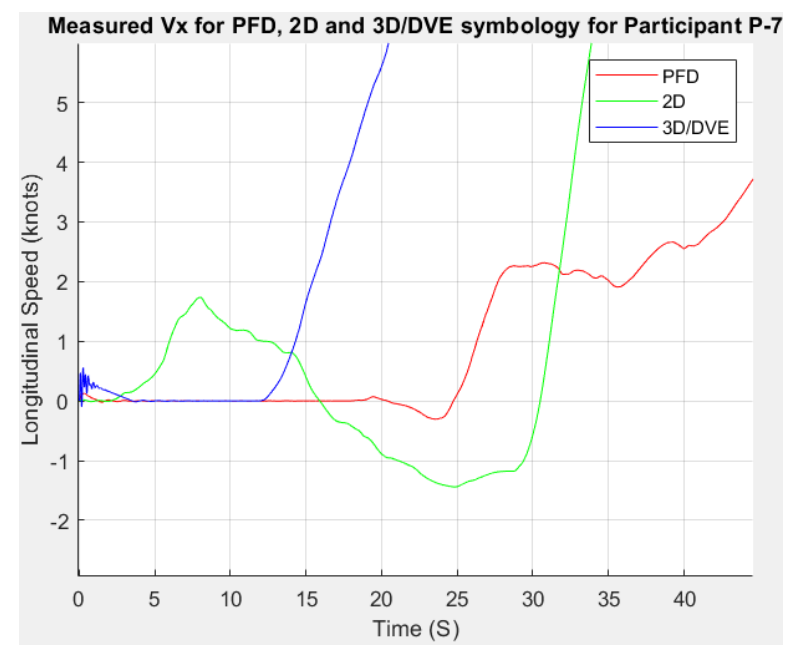

FiguRE A-19: MEASURED LONGITUdINAL SPEED FOR PARTICIPANT 7 IN HOVER. 


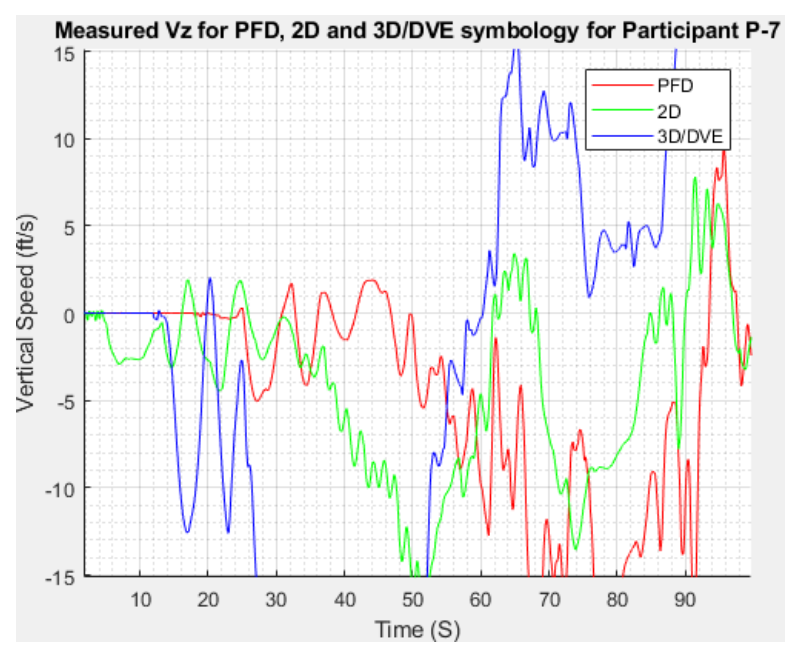

Figure A-20: MEASURED VERTICAL SPEED FOR

PARTICIPANT 7 IN HOVER.

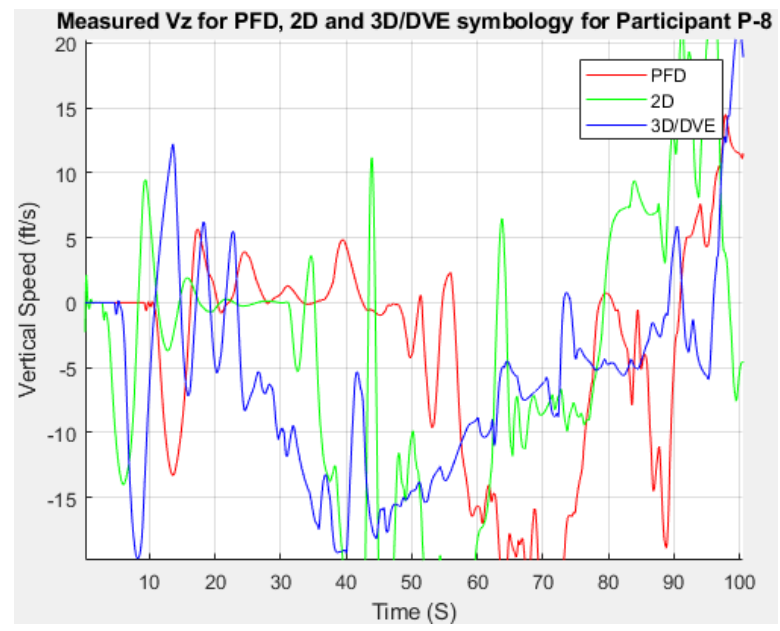

FIGURE A-21: MEASURED VERTICAL SPEED FOR PARTICIPANT 8 IN HOVER.

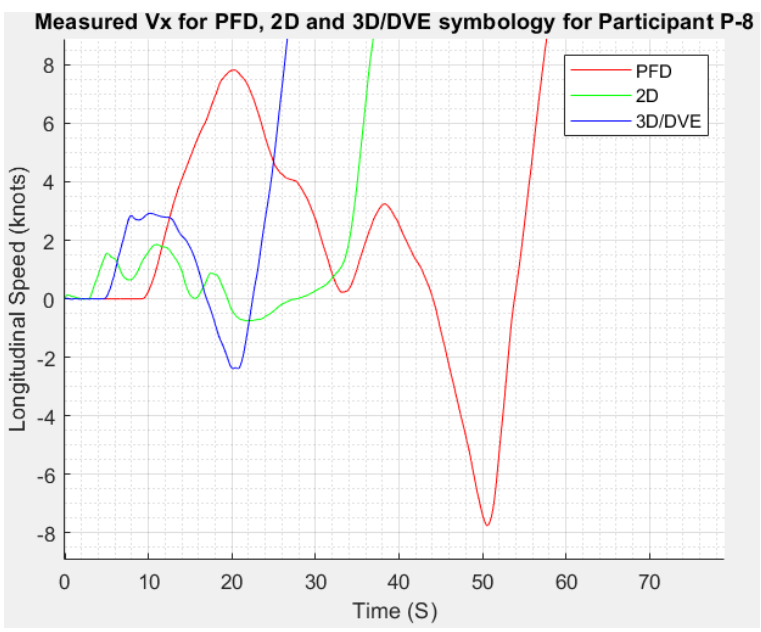

FIGURE A-22: MEASURED LONGITUDINAL SPEED FOR PARTICIPANT 8 IN HOVER.

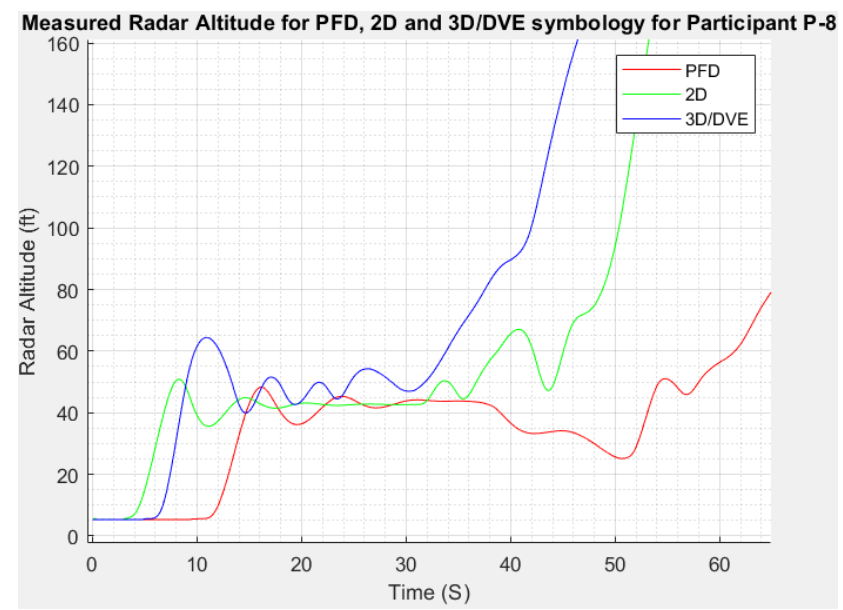

FIGURE A-23: MEASURED RADAR ALTITUDE FOR PARTICIPANT 8 IN HOVER. 


\section{APPENDIX B: CODES FOR DEVELOPMENT 3D Symbology OpenGL code}

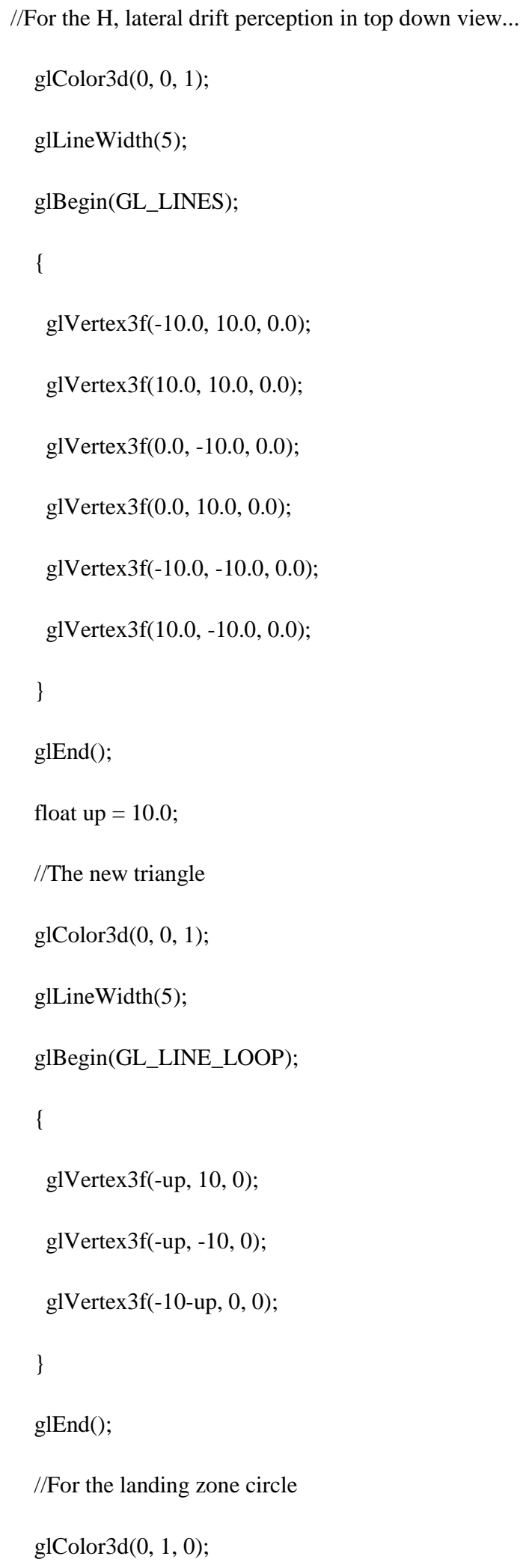


glLineWidth(8);

DrawEllipse(0, 0, 30, 23, 150);

// Left and Right Bars

glColor3d(0, 0, 1);

float $1 x=-15,1 y=-15,1 z=0$;

glBegin(GL_POLYGON);

\{

glVertex3f(lx + 0.5f, ly $-0.5 f, 1 z+20)$

glVertex3f(lx + 0.5f, ly + 0.5f, lz +20$)$

glVertex3f(lx $-0.5 f, 1 y+0.5 f, 1 z+20)$

glVertex3f(lx - 0.5f, ly - 0.5f, lz + 20);

glVertex3f(lx + 0.5f, ly $-0.5 f, 1 z-0.5 f)$

glVertex3f(lx + 0.5f, ly + 0.5f, lz - 0.5f)

glVertex3f(lx + 0.5f, ly + 0.5f, lz +20$)$

glVertex3f(lx + 0.5f, ly $-0.5 f, 1 z+20)$

glVertex3f(lx - 0.5f, ly - 0.5f, lz + 20);

glVertex3f(lx - 0.5f, ly + 0.5f, lz + 20)

glVertex3f(lx - 0.5f, ly + 0.5f, lz - 0.5f);

glVertex3f(lx - 0.5f, ly - 0.5f, lz - 0.5f);

glVertex3f(lx + 0.5f, ly + 0.5f, lz + 20)

glVertex3f(lx + 0.5f, ly + 0.5f, lz $-0.5 f)$

glVertex3f(lx - 0.5f, ly + 0.5f, lz - 0.5f);

glVertex3f(lx $-0.5 f, 1 y+0.5 f, 1 z+20)$

glVertex3f(lx + 0.5f, ly $-0.5 f, 1 z-0.5 f)$ 


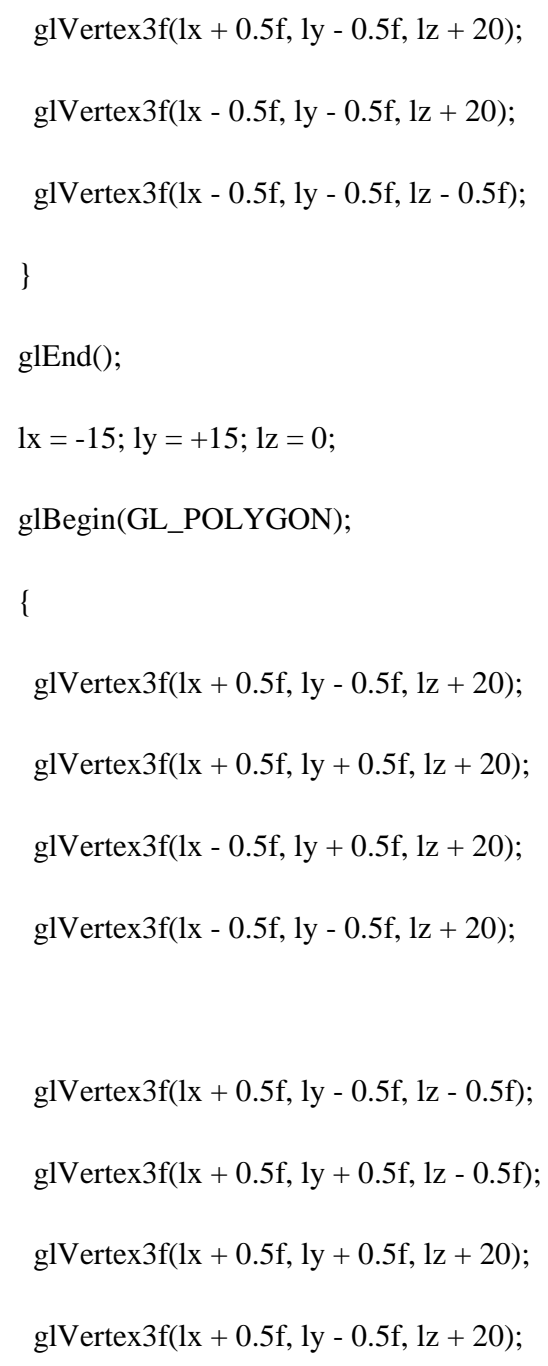




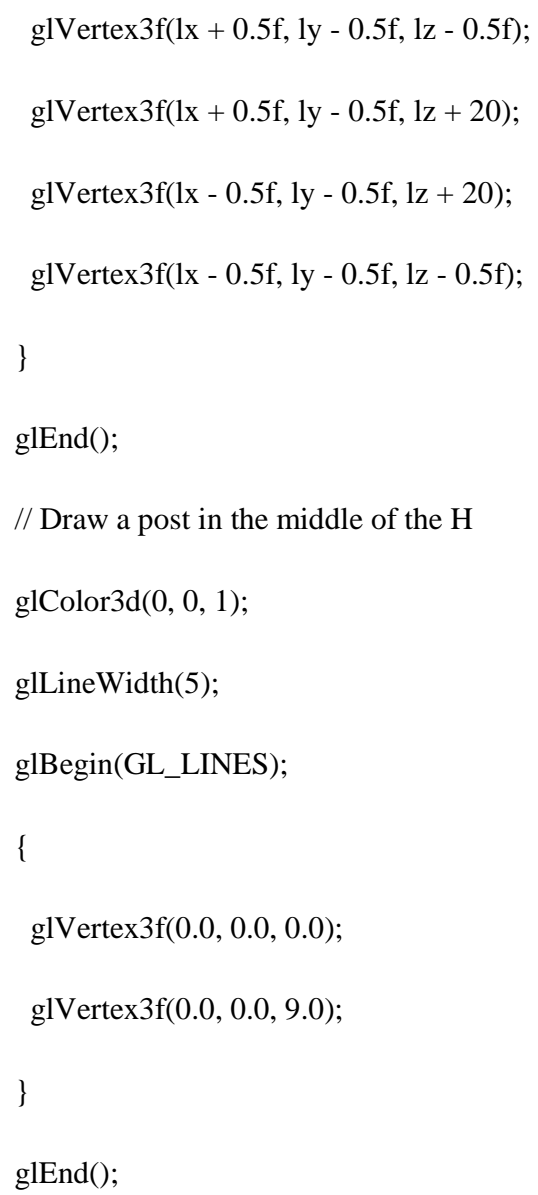

Dataflow between simulation (HeliSIM) and VAPS XT

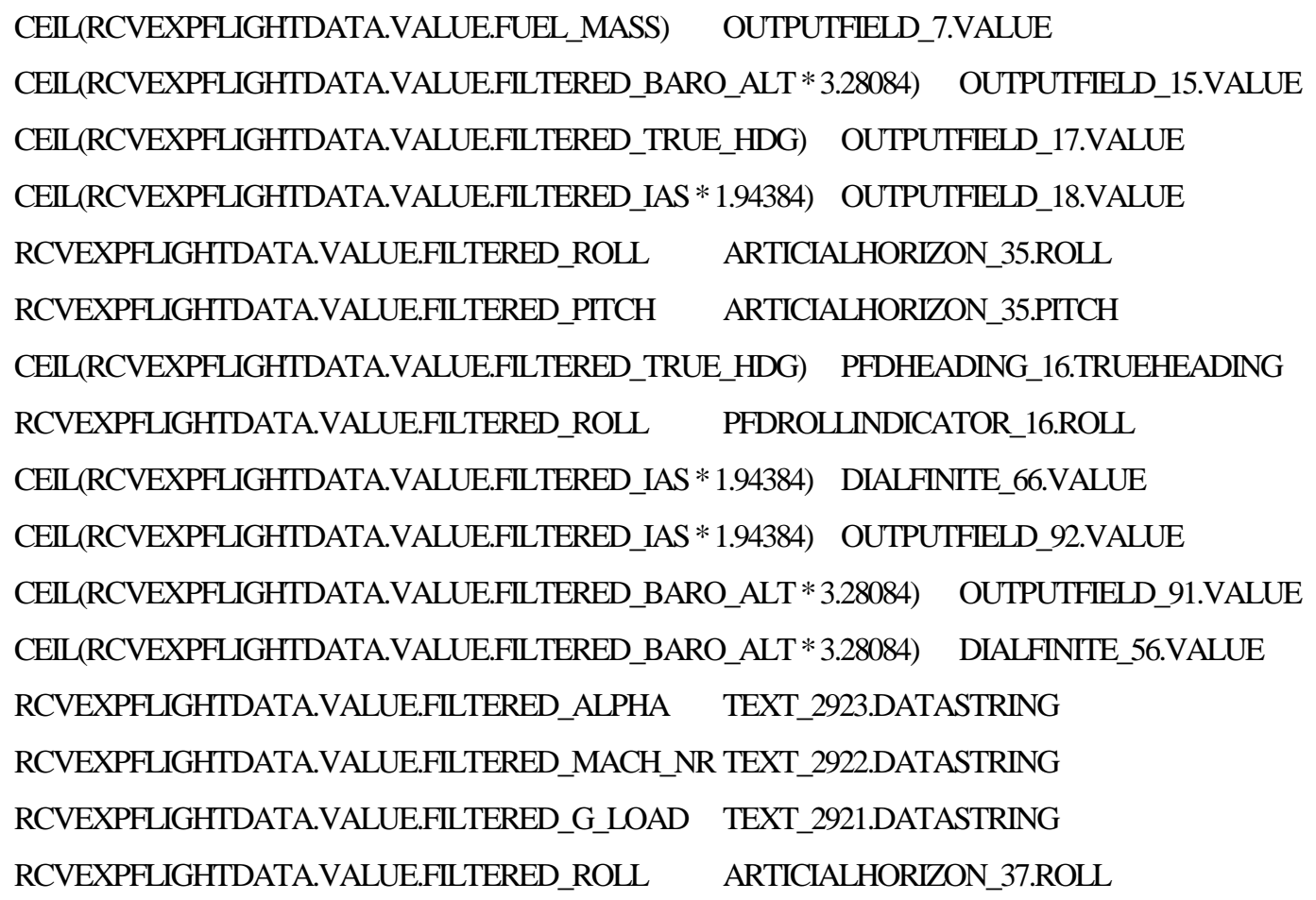


RCVEXPFLIGHTDATA.VALUE.FILTERED_PITCH ARTICIALHORIZON_37.PITCH

CEIL(RCVEXPFLIGHTDATA.VALUE.FILTERED_TRUE_HDG) PFDHEADING_18.TRUEHEADING

RCVEXPFLIGHTDATA.VALUE.FILTERED_ROLL PFDROLLINDICATOR_18.ROLL

CEIL(RCVEXPFLIGHTDATA.VALUE.FILTERED_BARO_ALT *3.28084) OUTPUTFIELD_42.VALUE

CEIL(RCVEXPFLIGHTDATA.VALUE.FILTERED_BARO_ALT*3.28084) DIALFINITE_65.VALUE

CEIL(RCVEXPFLIGHTDATA.VALUE.FILTERED_IAS * 1.94384) DIALFINITE_58.VALUE

CEIL(RCVEXPFLIGHTDATA.VALUE.FILTERED_IAS * 1.94384) OUTPUTFIELD_36.VALUE

rcvExpFlightData.Value.filtered_alpha Text_72.DataString

rcvExpFlightData.Value.filtered_mach_nr Text_54.DataString

rcvExpFlightData.Value.filtered_g_load Text_53.DataString

rcvEng_Info.Value.thrust_1_ Engine1_25.Value

rcvEng_Info.Value.throttle_1 Engine1_51.Value

rcvEng_Info.Value.oil_pres_1 Engine1_54.Value

rcvExpFlightData.Value.filtered_roll_ArticialHorizon_12.Roll

rcvExpFlightData.Value.filtered_pitch ArticialHorizon_12.Pitch

rcvExpFlightData.Value.filtered_roll_DialFinite_20.Value

Ceil(rcvExpFlightData.Value.filtered_true_hdg) DialCircular_19.Value

Ceil(rcvExpFlightData.Value.filtered_true_hdg) Text_924.DataString

Ceil(rcvExpFlightData.Value.filtered_baro_alt*3.28084) DialCircular_20.Value

Ceil(rcvExpFlightData.Value.filtered_baro_alt *3.28084) OutputField_45.Value

Ceil(rcvExpFlightData.Value.filtered_ias * 1.94384) DialFinite_21.Value

Ceil(rcvExpFlightData.Value.filtered_ias * 1.94384) OutputField_46.Value

rcvExpFlightData.Value.filtered_roll_ArticialHorizon_13.Roll

rcvExpFlightData.Value.filtered_pitch ArticialHorizon_13.Pitch

rcvExpFlightData.Value.filtered_roll DialFinite_22.Value

Ceil(rcvExpFlightData.Value.filtered_true_hdg) DialCircular_21.Value

Ceil(rcvExpFlightData.Value.filtered_true_hdg) Text_975.DataString

Ceil(rcvExpFlightData.Value.filtered_ias * 1.94384) DialFinite_23.Value

Ceil(rcvExpFlightData.Value.filtered_ias * 1.94384) OutputField_47.Value

Ceil(rcvExpFlightData.Value.filtered_baro_alt *3.28084) DialCircular_22.Value 


\section{APPENDIX C: RYERSON ETHICS BOARD APPROVAL AND RELATING DOCUMENTS \\ REB Approval Letter}

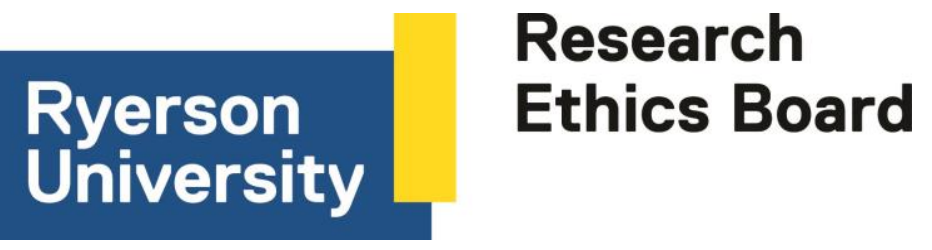

To: Vamshi Chittaluri Aerospace Engineering

Re: REB 2018-471: Development and evaluation of cueing methods by applying aircrew error taximony for Rotorcraft operations in Degraded Visual Environment (DVE) Date:February 11, 2019

Dear Vamshi Chittaluri,

The review of your protocol REB File REB 2018-471 is now complete. The project has been approved for a one year period. Please note that before proceeding with your project, compliance with other required University approvals/certifications, institutional requirements, or governmental authorizations may be required.

This approval may be extended after one year upon request. Please be advised that if the project is not renewed, approval will expire and no more research involving humans may take place. If this is a funded project, access to research funds may also be affected.

Please note that REB approval policies require that you adhere strictly to the protocol as last reviewed by the REB and that any modifications must be approved by the Board before they can be implemented. Adverse or unexpected events must be reported to the REB as soon as possible with an indication from the Principal Investigator as to how, in the view of the Principal Investigator, these events affect the continuation of the protocol.

Finally, if research subjects are in the care of a health facility, at a school, or other institution or community organization, it is the responsibility of the Principal Investigator to ensure that the ethical guidelines and approvals of those facilities or institutions are obtained and filed with the REB prior to the initiation of any research.

Please quote your REB file number (REB 2018-471) on future correspondence. 
Congratulations and best of luck in conducting your research.

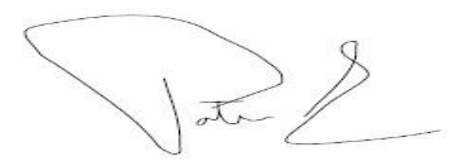

Dr. Patrizia Albanese, PhD

Chair, Ryerson University Research Ethics Board

The Following protocol attachments have been reviewed and approved.

- Bedford Workload Scale.docx (submitted on: 11 Dec 2018)

- NASA Task Load Index.docx (submitted on: 11 Dec 2018)

- Questionnaire to Complete.docx (submitted on: 12 Dec 2018)

- ConsentForm.docx (submitted on: 05 Feb 2019)

- Consent Preamble.docx (submitted on: 05 Feb 2019)

Recruitement Email.docx (submitted on: 09 Feb 2019)

REB 2018-471_Questions.docx (submitted on: 09 Feb 2019)

If any changes are made to the attached document throughout the course of the research, an amendment MUST be submitted to, and subsequently approved by the REB.

\section{REB Questionnaire (To be completed by Test participants)}

The NASA TLX is a multidimensional scale that rates overall pilot workload and stress based on six major factors.

Mental demand (Low/High): How much mental and perceptual activity is required for thinking, deciding, calculating and remembering or searching certain information that is being presented.

Physical Demand (Low/High): How much physical activity was required, pushing, pulling, turning, controlling activating, etc. to ideally plot to see whether a task was easy or strenuous.

Temporal Demand (Low/High): How much pressure does the operator feel due to the rate or pace at which the tasks or task elements occurred. 
Performance (Good/poor): How successful was the operator in accomplishing the goals of a given task set by the experimenter.

Effort (Low/High): How hard did the operator have to work both mentally and physically to accomplish the level of performance.

Frustration level (Low/High): How insecure, discouraged, irritated stressed and annoyed versus secure, gratified, content, relaxed and complacent did the operator feel with the information presented.

For the Experience you've just had on the Presagis HELI CRAFT simulator, please rate each individual component for how much demand the completion of the task required on a scale of 1 to 10 . This will provide the researcher with a qualitative idea of the Mental, Physical, Temporal, Performance demands and effort and frustration experienced in the completion of the task.

Mental Demand: Did the landing/takeoff require Low, medium or High (Provide number b/w 1 to 10) mental and perceptual activity for thinking, deciding, calculating, remembering or searching for certain information.

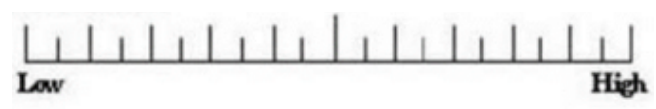

Physical Demand: Did the landing/takeoff require low, medium or high physical activity in controlling the helicopter? Adjusting the collective and cyclic more excessively.

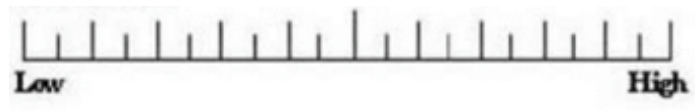

Temporal Demand: Was there low, medium or high pressure in the completion of the landing or takeoff sections of the flight scenario. Did you feel the pace of events was slow, medium or fast?

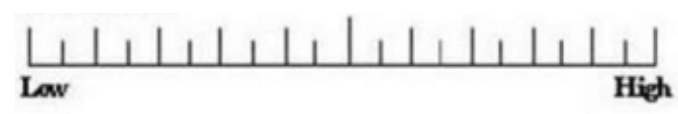

Effort: Did you feel you had to work hard or not for the accomplishment of the goal at hand? Mentally and Physically? Please rate on a scale of 1 to 10, 1 being minimal effort, 10 being high effort.

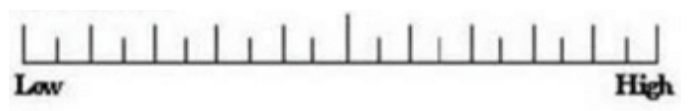


Performance: Did you feel that landing or take off or the general performance of the task to be good or poor (Rate on a scale of 1 to 10)? Did you feel any limitations derailed you achieving an objective? Do you see any improvement that can help you perform better?

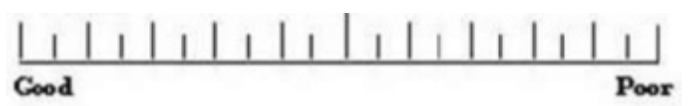

Frustration: In the completion of the landing or takeoff task, did you feel high or low levels of frustration?

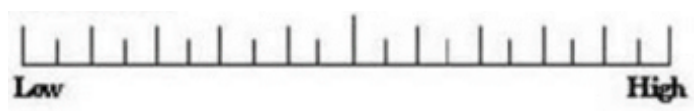

Please provide any comments that you may have as a suggestion:

Please answer the following questions via following the chart to providing an understanding of the workload demand you felt. 


\section{Bedford Workload Scale}

OPERATOR DEMAND LEVEL RATING

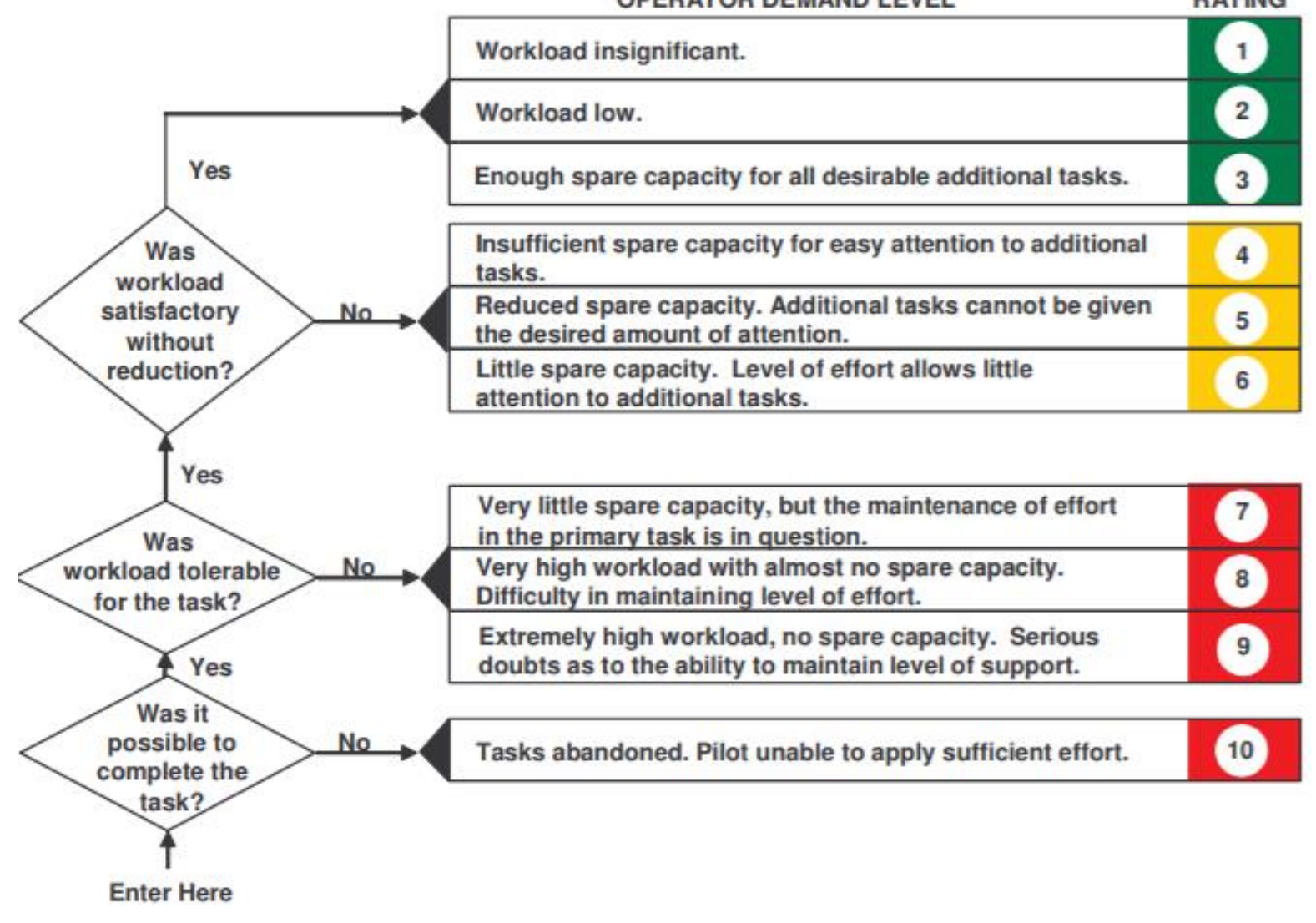

Overall, please specify if you see value in the implementation of the DVENS system and the associating symbology. Please provide any suggestions you may have with regards to the implementation. 


\section{REB Consent Agreement}

\section{Ryerson University}

\section{Consent Agreement}

You are being invited to participate in a research study. Please read this consent form so that you understand what your participation will involve. Before you consent to participate, please ask any questions to be sure you understand what your participation will involve.

TITLE OF THE STUDY: Development and evaluation of cueing methods by applying aircrew error taximony for Rotorcraft operations in Degraded Visual Environment (DVE)

INVESTIGATORS: This research study is being conducted by Vamshi Chittaluri, under the supervision of Dr. Joon Chung, the Department of Aerospace Engineering at Ryerson University.

This study is funded by CRIAQ (CONSORTIUM FOR RESEARCH AND INNOVATION IN AEROSPACE IN QUÉBEC)

If you have any questions or concerns about the research, please feel free to contact Vamshi Chittaluri at vamshi.chittaluri@ryerson.ca.

PURPOSE OF THE STUDY: As part of the researcher's Master of Applied Science (MASc) thesis, Vamshi Chittaluri would like to obtain results on the study of improvement in pilot performance through the application of DVENS system. The pilot workload, stress and time load will be collected through nonintrusive methods.

WHAT YOU WILL BE ASKED TO DO: As a participant in this research, you will be asked to fly a helicopter flight simulator. You will be tasked with flying the simulator through various test scenarios and provide feedback on operational and visual comfort of the flight symbology used during the simulation. You will be asked to fill in questionnaires that help measure the workload, stress and time that task competition requires. Each simulation will last for approximately twenty minutes and will be conducted at the Presagis Headquarters in Quebec. As a participant of this test you will have access to the results of your testing by contacting the investigators via email.

POTENTIAL BENEFITS: I cannot guarantee, however, that you will receive any benefits from participating in this study. You will be contributing to research and future development efforts.

WHAT ARE THE POTENTIAL RISKS TO YOU AS A PARTICIPANT: There are very low risks attached to the participation in this research. As a participant you make experience some visual discomfort while operating the simulator, at which point you may stop participating, either temporarily or 
permanently. In the event of an erratic flight maneuver the you might feel discomfort due to rapidly changing graphics and motion.

CONFIDENTIALITY: The collected data from the experiment will only be summarized and presented in the form of a thesis and research papers. The participant's name will not be listed in any of the research papers or thesis, unless permission is granted, you will be acknowledged for your contribution to the research. The data will be stored on Ryerson's google drive and is secure with encryption. Data will be destroyed once the all raw data is summarized. The summarized data will be presented in the form of an MASc thesis and will be available to all participant at their request.

VOLUNTARY PARTICIPATION AND WITHDRAWAL: Participation in this study is completely voluntary. You can choose whether to be in this study or not. If any question makes you uncomfortable, you can skip that question. You may stop participating at any time and you will still be given the incentives and reimbursements described above. If you choose to stop participating, you may also choose to not have your data included in the study. Your choice of whether or not to participate will not influence your future relations with Ryerson University or the investigators, Vamshi Chittaluri, involved in the research.

Some of the questions you may be asked are:

1. On a scale of 1 to 10 , how demanding was the task? 1 being not demanding at all and 10 being extremely demanding.

QUESTIONS ABOUT THE STUDY If you have any questions about the research now, please ask. If you have questions later about the research, you may contact:

\section{Vamshi Chittaluri}

MASc Graduate Student Aerospace Engineering vamshi.chittaluri@ryerson.ca

\section{Dr. Joon Chung}

Professor of Aerospace Engineering at Ryerson University

416-979-5000 ext. 7213

j3chung@ryerson.ca

This study has been reviewed by the Ryerson University Research Ethics Board. If you have questions regarding your rights as a participant in this study, please contact:

Research Ethics Board 
c/o Office of the Vice President, Research and Innovation

Ryerson University

350 Victoria Street

Toronto, ON M5B 2K3

416-979-5042

rebchair@ryerson.ca

Development and evaluation of cueing methods by applying aircrew error taximony for Rotorcraft operations in Degraded Visual Environment (DVE)

CONFIRMATION OF AGREEMENT

Your signature below indicates that you have read the information in this agreement and have had a chance to ask any questions you have about the study. Your signature also indicates that you agree to participate in the study and have been told that you can change your mind and withdraw your consent to participate at any time. You have been given a copy of this agreement. You have been told that by signing this consent agreement you are not giving up any of your legal rights.

Name of Participant (please print)

Signature of Participant

Date 


\section{REFERENCES}

[1] W. Greg, Fatal Traps for Helicopter Pilots. McGraw-Hill Professional Publishing, 2006.

[2] "BELL HUEY (Online)." [Online]. Available: https://www.tumblr.com/search/bell huey.

[3] D. W. Albery et al., "Rotary-Wing Brownout Mitigation: Technologies and Training ( Remèdes contre le phénomène de brownout," 2012.

[4] H. to keep a helicopter steady when close to the ground? (Online), "How to keep a helicopter steady when close to the ground? (Online)." [Online]. Available:

https://aviation.stackexchange.com/questions/46268/how-to-keep-a-helicopter-steady-when-closeto-the-ground. [Accessed: 23-Jul-2019].

[5] “The Guardian: Degraded Visual Environment.” [Online]. Available: https://www.theguardian.com/news/datablog/2009/jul/13/afghanistan-polls, http://www.dailymail.co.uk/news/article-1346741/Bloomberg-says-New-York-beaten-snowweather-bomb-dumps-9-inches-Central-Park.html.

[6] M. Higginbotham, "Flightfax," Flightfax, U.S Army Combat Readiness Cent., p. 99, 2015.

[7] F. Street, A. Mckinley, and D. Ph, "Sensor-Based Technology for Rotary Wing Aircraft in Low Visibility Environments," pp. 0-9.

[8] Franz Xaver Viertler, "Visual Augmentation for Rotorcraft Pilots in Degraded Visual Environment: PhD Thesis," Munich, 2017.

[9] G. M. Neiswander, "Improving rotorcraft deceleration guidance for brownout landing: Master of Science Thesis," University of Iowa, 2010.

[10] G. Roy, X. Cao, R. Bernier, and S. Roy, "Enhanced scanning agility using a double pair of Risley prisms," Appl. Opt., vol. 54, no. 34, p. 14, 2015.

[11] G. Roy, S. Roy, and X. Cao, "Helicopter Flight Test of the Obscurant Penetrating Autosynchronous Lidar ( OPAL ) in Degraded Visual Environments : Part II - See-through Capability Assessment Lidar consultant," AHS 70th Annu. Forum, pp. 1-7, 2015.

[12] P. Cae, "Presagis HeliSIM.” [Online]. Available: https://www.presagis.com/en/product/helisim/.

[13] P. Cae, "Presagis HeliCraft." [Online]. Available: https://www.presagis.com/en/product/heli-craft/. [Accessed: 14-Jul-2019]. 
[14] C. Presagis, "Presagis VegaPrime." [Online]. Available:

https://www.presagis.com/en/product/vega-prime/.

[15] P. Cae, "Presagis VapsXT." [Online]. Available: https://www.presagis.com/en/product/vaps-xt/. [Accessed: 14-Jul-2019].

[16] C. Presagis, "No Title.” [Online]. Available: https://www.presagis.com/en/product/ondulus-ir/. [Accessed: 21-Jul-2019].

[17] C. Presagis, "No Title.” [Online]. Available: https://www.presagis.com/en/glossary/detail/lidar/. [Accessed: 30-Jul-2019].

[18] J. Kessenich, D. Baldwin, and R. Rost, "The OpenGL Shading Language (Version 4.10)," Language (Baltim)., 2010.

[19] J. Vreeken, H. Haverdings, and M. Joosse, "Helicopter flight in a degraded visual environment," 2013.

[20] D. G. Newman, An overview of spatial disorientation as a factor in aviation accidents and incidents. 2007.

[21] D. A. Wiegmann and S. A. Shappell, A human error approach to aviation accident analysis, 1 edition. Taylor and Francis, 2016.

[22] R. H. Hoh, "The Effects of Degraded Visual Cueing and Divided Attention on Obstruction Avoidance in Rotorcraft," Washington, DC/ Lomita, CA, 1990.

[23] C. D. Wickens, "Situation awareness and workload in aviation," Curr. Dir. Psychol. Sci., vol. 11, pp. 128-133, 2002.

[24] M. Martinussen and David R. Hunter, Aviation Psychology and Human Factors, 2nd ed. CRC Press; 1 edition (Aug. 30 2007), 2011.

[25] “IAI Reveals its New Helicopter Safety Technology (Online).” [Online]. Available: https://www.israeldefense.co.il/en/content/iai-reveals-its-new-helicopter-safety-technology. [Accessed: 23-Jul-2019].

[26] S. Coyle, "Helicopter Flying Handbook: Advanced Flight Maneuvers," in Helicopter Flying Handbook, Oklahoma City: Federal Aviation Administration, 2012, p. 200.

[27] Y. Lim et al., "Avionics Human-Machine Interfaces and Interactions for Manned and Unmanned Aircraft," Prog. Aerosp. Sci., vol. 102, pp. 1-46, 2018. 
[28] D. Minotra and K. Feigh, "Eliciting knowledge from helicopter pilots: Recommendations for revising the ACTA method for helicopter landing tasks," Proc. Hum. Factors Ergon. Soc., vol. 2017-Octob, no. Cdm, pp. 242-246, 2017.

[29] T. W. Eger, "Operational requirements for short-term solution in visual display specifically for Degraded Visual Environment (DVE)," SPIE, vol. 8360, p. 83600L, 2012.

[30] R. J. Nichol, “Airline Head-Up Display Systems: Human Factors Considerations,” Int. J. Econ. Manag. Sci., vol. 04, no. 05, 2015.

[31] “ILS Localiser (Online)." [Online]. Available: https://en.wikipedia.org/wiki/Instrument_landing_system_glide_path\#/media/File:ILS_localizer_il lustration.svg. [Accessed: 23-Jul-2019].

[32] J. P. Brown, "The Effect of Automation on Human Factors in Aviation," J. Instrumentation, Autom. Syst., vol. 3, no. 2, pp. 31-46, 2018.

[33] J. Liu, A. Gardi, S. Ramasamy, Y. Lim, and R. Sabatini, "Cognitive pilot-aircraft interface for single-pilot operations," Knowledge-Based Syst., vol. 112, pp. 37-53, 2016.

[34] W. R. E. Fred H Previc, Spatial Disorientation in Aviation - Progress in Astronautics and Aeronautics. American Institute of Aeronautics and Astronautics, 2004.

[35] D. P. Veillette, "Helicopter Flying Handbook: Attitude Instrument Flying," in Helicopter Flying Handbook, Oklahoma: Federal Aviation Administration, 2012, p. 200.

[36] U. S. F. A. A. (FAA), “Airplane Attitude Instrument Flying Using an Electronic Display,” in Pilot's Handbook of Aeronautical Knowledge, Oklahoma City: United States Department of Transportation, Federal Aviation Administration, Airman Testing Standards Branch, 2008, pp. 4.1-4.14.

[37] J. M. Reising and K. K. Liggett, "New flight display formats," no. 937, pp. 86-91, 1995.

[38] G. Costa, Fatigue and Biological Rhythms. 2010.

[39] J. R. R. Stott, “Orientation and disorientation in aviation,” Extrem. Physiol. Med., vol. 2, no. 1, pp. 1-11, 2013.

[40] S. H. Kim et al., "Multidimensional measure of display clutter and pilot performance for advanced head-up display," Aviat. Sp. Environ. Med., vol. 82, no. 11, p. 10, 2011.

[41] P. M. Knabl, H.-U. Doehler, S. Schmerwitz, and M. Biella, "Integration of a helmet-mounted 
display for helicopter operations in degraded visual environment: a human factors perspective," pp. $978-0,2012$.

[42] H.-U. Doehler, S. Schmerwitz, and T. Lueken, "Visual-conformal display format for helicopter guidance," no. May, p. 13, 2014.

[43] T. Münsterer, M. Kress, and S. Klasen, "Sensor based 3D conformal cueing for safe and reliable HC operation specifically for landing in DVE," vol. 8737, p. 87370A, 2013.

[44] Z. P. Szoboszlay, R. A. McKinley, L. S. R. Braddom, W. W. Harrington, H. N. Burns, and J. C. Savage, "Landing an H-60 helicopter in brownout conditions using 3D-LZ displays," Am.

Helicopter Soc. 66th Annu. Forum, Pheonix, no. May 2010, 2010.

[45] J. M. A. Ricciardi, "The Effects of Terrain Discrepancy and the Role of Texture in Enhanced/Synthetic Vision Systems," University of Toronto, 2000.

[46] L. J. Prinzel and L. J. Kramer, "Synthetic vision systems," 2018.

[47] L. Velisavljević and J. H. Elder, "Texture properties affecting the accuracy of surface attitude judgements," Vision Res., vol. 46, no. 14, pp. 2166-2191, 2006.

[48] R. Rosenholtz and J. Malik, "Surface Orientation from Texture: Isotropy or Homogeneity (or Both)? INTRODUCTION: TWO MODELS FOR SHAPE FROM TEXTURE,”Vis. Res, vol. 37, no. 16, pp. 2283-2293, 1997.

[49] A. P. Witkin, "Recovering surface shape and orientation from texture," Artif. Intell., vol. 17, no. 1-3, pp. 17-45, 1981.

[50] S. Kim, H. Hagh-Shenas, and V. Interrante, "Showing Shape with Texture: Two Directions Seem Better than One," Proc. Hum. Vis. Electron. Imaging VIII, vol. 5007, pp. 332-339, 2003.

[51] S. Kim, H. Hagh-Shenas, and V. Interrante, "Conveying shape with texture: An experimental investigation of the impact of texture type on shape categorization judgments," Proc. - IEEE Symp. Inf. Vis. INFO VIS, pp. 163-172, 2003.

[52] T. Butkiewicz and A. H. Stevens, "Effectiveness of Structured Textures on Dynamically Changing Terrain-like Surfaces," IEEE Trans. Vis. Comput. Graph., vol. 22, no. 1, pp. 926-934, 2016.

[53] J. Haber, "Enhancing the functional design of a multi-touch UAV Ground Control Station.," Ryerson University, 2013.

[54] T. Amida, "DESIGN AND EVALUATION OF UAV GROUND CONTROL STATION BY 
CONSIDERING HUMAN FACTORS STANDARDS AND GUIDELINES,” Ryerson University, 2017.

[55] M. Maguire, "Methods to support human-centred design," Int. J. Hum. Comput. Stud., vol. 55, no. 4, pp. 587-634, 2001.

[56] I. N. S. interdisciplinaire de Normalisation, "Ergonomics and Human-System Interaction-Part 210: Human-Centred Design for interactive systems (ISO 9241-210:2010),” 2017.

[57] Y. Cao, M. Theune, and A. Nijholt, Towards cognitive-aware multimodal presentation: The modality effects in high-load HCI, vol. 5639 LNAI. 2009.

[58] C. R. C. P. Llc, Handbook of Human Factors and Ergonomics Methods. 2004.

[59] S. Gudipati and A. Pennathur, "Physical and mental workload assessment techniques for job design,” El Paso, 2015.

[60] S. M. Casner and B. F. Gore, "Measuring and Evaluating workload: A Primer," Hanover, MD, 2010.

[61] A. H. Roscoe and G. Ellis, "A subjective rating scale for assessing pilot workload in flight: A decade of practical use," R. Aircr. Establ. TR 90019, p. 18, 1990.

[62] D. D. Haiyan Liu, Xianchao Ma, Yinbo Zhang, Zhefeng Jin, "A Practice for the Certification of Minimum Flight Crew Workload," Springer Professional. [Online]. Available:

https://www.springerprofessional.de/a-practice-for-the-certification-of-minimum-flight-crewworkload/12759680.

[63] H. Graham, M. L. Cummings, M. A. I. Nstitute, and O. F. T. Echnology, "MODIFIED COOPER HARPER SCALES FOR ASSESSING UNMANNED VEHICLE DISPLAYS,” Cambridge, MA, 2008.

[64] M. T. Di Palo, "Rating Satisfaction Research : Is it Poor, Fair, Good, Very good or Excellent," Amcrican Coll. Rheumatol., vol. 10, no. 6, pp. 422-430, 1997.

[65] R. V Parrish et al., "Aspects of synthetic vision display systems and the best practices of the NASA's SVS project," no. May, pp. 1-162, 2008.

[66] P. M. Ververs and C. D. Wickens, "Conformal Flight Path Symbology for Head-Up Displays: Defining the Distribution of Visual Attention in Three-Dimensional Space,” no. August, pp. 1$118,1998$. 
[67] “Flight Path Angle [Image]." [Online]. Available: http://code7700.com/v-x.htm. [Accessed: 14Jul-2019].

[68] P. H. Hall, "A Set of Flight Dynamic Equations for Aircraft Simulation.," Melbourne, 1982.

[69] "Forces on an Aircraft (Image/Online)." [Online]. Available: http://www.faatest.com/books/FLT/Chapter17/ClimbPerformance.htm. [Accessed: 14-Jul-2019].

[70] “SideSlip Indicator [Image].” [Online]. Available: https://forum.il2sturmovik.com/topic/16246slip-indicators-correlate-to-wind-speed-direction-not-to-aircraft-lateral-acceleration-as-they-oughtto/.

[71] “Sideslip Equation [Image]." [Online]. Available: https://aviation.stackexchange.com/questions/21671/how-does-the-aspect-ratio-of-the-verticalstabilizer-affect-stall-speed/21708\#21708. [Accessed: 14-Jul-2019].

[72] M. Tushar, S. Singdha, and A. Zakir, "Design and comparison of butterworth and chebyshev type1 low pass filter using Matlab,” Res. Cell An Int. J. Eng. Sci., vol. 4, 2011.

[73] S. Smith, S. Jennings, and G. Craig, "Degraded Visual Environment Navigation System ( DVENS ) Symbology Description : 2D Symbology,” 2017.

[74] A. Anisya and G. Y. Swara, "Implementation of Haversine Formula and Best First Search Method in Searching of Tsunami Evacuation Route," IOP Conf. Ser. Earth Environ. Sci., vol. 97, no. 1, 2017.

[75] S. Hartanto, M. Furqan, A. Putera, U. Siahaan, and W. Fitriani, "Haversine Method in Looking for the Nearest Masjid," Int. J. Recent Trends Eng. Res., vol. 3, no. 8, pp. 187-195, 2017.

[76] S. Gopal, K. Patro, and K. Kumar, "Normalization: A Preprocessing Stage," Int. Adv. Res. J. Sci. Eng. Technol., vol. 2, no. 3, p. 4, 2015.

[77] Z. P. Szoboszlay, T. S. Turpin, and R. A. Mckinley, "Symbology for Brown-Out Landings : The First Simulation for the 3D-LZ Program," 65th Am. Helicopter Soc. Annu. Forum 2009, no. May 2009, 2009.

[78] F. Erazo and P. Affairs, "Display Scaling of Degraded Visual Environment Helicopter Symbology in Station-Keeping Tasks by," 2017. 